University of Louisville

ThinkIR: The University of Louisville's Institutional Repository

$5-2013$

\title{
Nanocarbon/elastomer composites : characterization and applications in photo-mechanical actuation.
}

Robert James Loomis 1978-

University of Louisville

Follow this and additional works at: https://ir.library.louisville.edu/etd

\section{Recommended Citation}

Loomis, Robert James 1978-, "Nanocarbon/elastomer composites : characterization and applications in photo-mechanical actuation." (2013). Electronic Theses and Dissertations. Paper 855.

https://doi.org/10.18297/etd/855

This Doctoral Dissertation is brought to you for free and open access by ThinkIR: The University of Louisville's Institutional Repository. It has been accepted for inclusion in Electronic Theses and Dissertations by an authorized administrator of ThinkIR: The University of Louisville's Institutional Repository. This title appears here courtesy of the author, who has retained all other copyrights. For more information, please contact thinkir@louisville.edu. 
NANOCARBON/ELASTOMER COMPOSITES:

CHARACTERIZATION AND APPLICATIONS IN PHOTO-MECHANICAL ACTUATION

\author{
By \\ Robert James Loomis III \\ B.S. University of Michigan, 2001 \\ M.S., Old Dominion University, 2007
}

\begin{abstract}
A Dissertation
Submitted to the Faculty of the Speed School of the University of Louisville in Partial Fulfillment of the Requirements for the Degree of

Doctor of Philosophy

Department of Mechanical Engineering

University of Louisville

Louisville, Kentucky
\end{abstract}

May 2013 

NANOCARBON/ELASTOMER COMPOSITES:

CHARACTERIZATION AND APPLICATIONS IN PHOTO-MECHANICAL ACTUATION

\author{
By \\ Robert James Loomis III \\ B.S. University of Michigan, 2001 \\ M.S., Old Dominion University, 2007 \\ A Dissertation Approval on
}

April 2, 2013

by the following Dissertation Committee

Dr. Balaji Panchapakesan, Dissertation Director

Dr. Robert W. Cohn

Dr. Thomas Berfield

Dr. Stuart Williams 


\section{DEDICATION}

To my loving wife Autumn, whose unwavering support and continuous words of encouragement were indispensable during my studies. 


\section{ACKNOWLEDGMENTS}

It has been both a pleasure as well as an extraordinary enlightening experience to study at the University of Louisville during the past few years. I am indebted to the many world-class instructors, mentors, and peers whom I've had the opportunity to become acquainted and work with throughout my studies. Several individuals, however, have had quite significant positive impacts on my education. These include my dissertation director, Dr. Balaji Panchapakesan, a gifted scholar with contagious enthusiasm who not only pushes his students to achieve on both personal as well as academic levels, but also someone who firmly believes in the value of education and proper preparation towards future career goals. Additionally, I'd like to thank my committee members, Dr. Robert Cohn, Dr. Stuart Williams, and Dr. Thomas Berfield; without their insightful feedback, direction, and advice this dissertation would not be possible. Finally, I'd like to thank my wonderful wife and parents for their constant support and many hours spent (especially my mom) helping proofread my papers. 


\section{ABSTRACT \\ NANOCARBON/ELASTOMER COMPOSITES: \\ CHARACTERIZATION AND APPLICATIONS IN PHOTO-MECHANICAL ACTUATION}

Robert James Loomis III

April 2, 2013

Materials that change shape or dimensions in response to external stimuli are widely used in actuation devices. While plenty of systems respond to heat, light, electricity, and magnetism, there is an emerging class of light-driven actuators based on carbon nanostructure/elastomer composites. The addition of nanomaterials to elastomeric polymers results not only in significant material property improvements such as mechanical strength, but also assists in creating entirely new composite functionalities as with photo-mechanical actuation. Efficient photon absorption by nanocarbons and subsequent energy transduction to the polymeric chains can be used to controllably produce significant amounts of pre-strain dependent motion. Photo-mechanical actuation offers a variety of advantages over traditional devices, including wireless actuation, electro-mechanical decoupling (and therefore low noise), electrical circuit elimination at point of use, massive parallel actuation of device arrays from single light source, and complementary metal-oxide-semiconductor / micro-electro-mechanical (CMOS/MEMS) compatible processing. Applications of photo-responsive materials encompass robotics, plastic motors, photonic switches, micro-grippers, and adaptive micro-mirrors. 
The magnitude and direction of photo-mechanical actuation responses generated in carbon nanostructure/elastomer composites depend on applied pre-strains. At low levels of pre-strains (3-9\%), actuators show reversible photo-induced expansion while at high levels (15-40\%), actuators exhibit reversible contraction. Large, light-induced reversible and elastic responses of graphene nanoplatelet (GNP) polymer composites were demonstrated for the first time, with an extraordinary optical-to-mechanical energy conversion factor $\left(\eta_{\mathrm{M}}\right)$ of 7-9 MPa/W. Following this demonstration, similar elastomeric composite were fabricated with a variety of carbon nanostructures. Investigation into photo-actuation properties of these composites revealed both layer-dependent, as well as dimensionally-dependent responses. For a given carbon concentration, both steady-state photo-mechanical stress response and energy conversion efficiency were found to be directly related to dimensional state of carbon nanostructure additive, with onedimensional (1D) carbon nanotubes demonstrating the highest responses ( $\sim 60 \mathrm{kPa}$ stress and $\sim 5 \times 10^{-3} \%$ efficiency at just $1 \mathrm{wt} \%$ loading) and three-dimensional (3D) highly ordered pyrolytic graphite demonstrating the lowest responses. Furthermore, development of an advanced dispersion technique (evaporative mixing) resulted in the ability to fabricate conductive composites. Actuation and relaxation kinetics responses were investigated and found to be related not to dimensionality, but rather the percolation threshold of carbon nanostructure additive in the polymer. Establishing a connective network of carbon nanostructure additive allowed for energy transduction responsible for photo-mechanical effect to activate carbon beyond the infrared (IR) illumination point, resulting in enhanced actuation. Additionally, in the conductive samples photoconductivity as a function of applied pre-strain was also measured. Photo-conductive 
response was found to be inversely proportional to applied pre-strain, demonstrating mechanical coupling.

Following investigation into photo-mechanical actuation responses between the various carbon forms, use of these composite actuators to achieve both macroscopic as well as microscopic movement in practical applications was evaluated. Using dual GNP/elastomer actuators, a two-axis sub-micron translation stage was developed, and allowed for two-axis photo-thermal positioning $(\sim 100 \mu \mathrm{m}$ per axis $)$ with $120 \mathrm{~nm}$ resolution (limitation of the feedback sensor) and $\sim 5 \mu \mathrm{m} / \mathrm{s}$ actuation speeds. A proportional-integral-derivative control loop automatically stabilizes the stage against thermal drift, as well as random thermal-induced position fluctuations (up to the bandwidth of the feedback and position sensor). Nanopositioner performance characteristics were found to be on par with other commercial systems, with resolution limited only by the feedback system used. A mathematical model was developed to describe the elastomeric composite actuators as a series of $n$ springs, with each spring element having its own independent IR-tunable spring constant. Effects of illumination intensity, position, and amount of the composite actuator illuminated are discussed. This model provided several additional insights, such as demonstrating the ability to place not just one, but multiple stages on a single polymer composite strip and position them independently from one another, a benefit not seen in any other type of positioning system.

Further investigation yielded interesting and novel photo-mechanical properties with actuation visible on macroscopic scales. Addition of a third component (thermally expanding microspheres), produced a new class of stimuli-responsive expanding polymer 
composites with ability to unidirectionally transform physical dimensions, elastic modulus, density, and electrical resistance. Carbon nanotubes and core-shell acrylic microspheres were dispersed in polydimethylsiloxane, resulting in composites that exhibit a binary set of material properties. Upon thermal or IR stimuli, liquid cores encapsulated within the microspheres vaporize, expanding the surrounding shells and stretching the matrix. Microsphere expansion results in visible dimensional changes, regions of reduced polymeric chain mobility, nanotube tensioning, and overall elastic to plastic-like transformation of the composite. Transformations include macroscopic volume expansion $(>500 \%)$, density reduction $(>80 \%)$, and elastic modulus increase $(>675 \%)$. Additionally, conductive nanotubes allow for remote expansion monitoring and exhibit distinct loading-dependent electrical responses.

Compared to well-established actuation technologies, research into photo-mechanical properties of carbon-based polymer composites is still in its infancy. Results in this dissertation demonstrate some of the enormous potential of light-driven carbon-based composites for actuation and energy scavenging applications. Furthermore, mechanical response dependence to carbon nanostructure dimensional state could have significance in developing new types of carbon-based mixed-dimensional composites for sensor and actuator systems. As the fabrication processes used here are compatible with CMOS and MEMS processing, carbon-based polymer composites allow for not only scaling actuation systems, but also ability to pattern regions of tailorable expansion, strength, and electrical resistance into a single polymer skin, making these composites ideal for structural and electrical building blocks in smart systems. Continued development of carbon-based polymer composites will extend the promising potential of 
light-driven actuation technologies and will serve as a catalyst to inspire continued research into energy conversion devices and systems. 
TABLE OF CONTENTS

PAGE

ACKNOWLEDGMENTS

iv

ABSTRACT

LIST OF FIGURES

$\mathrm{V}$

xiii

1 INTRODUCTION 1

2 BACKGROUND REVIEW 10

2.1 INTRODUCTION 10

2.2 CARBON NANOSTRUCTURES 11

2.3 MICRO/NANOACTUATOR TECHNOLOGIES 18

2.4 OPTICALLY DRIVEN ACTUATORS 26

3 GRAPHENE-NANOPLATELET-BASED PHOTO-MECHANICAL 34 ACTUATORS

3.1 INTRODUCTION 34

3.2 EXPERIMENTAL SETUP 35

3.3 PHOTO-MECHANICAL STRESS AND KINETICS 36

3.4 TEMPERATURE RELATIONSHIP 44

3.5 CONCLUSIONS 47

4 LAYER DEPENDENT MECHANICAL RESPONSES OF GRAPHENE 50 COMPOSITES TO NEAR-INFRARED LIGHT

4.1 INTRODUCTION 50

4.2 RESULTS 50

4.3 CONCLUSIONS 57

5 DIMENSIONAL DEPENDENCE OF PHOTO-MECHANICAL 58 RESPONSE 
5.1 INTRODUCTION 58

5.2 RESULTS $\quad 59$

5.2.1 STRESS RESPONSE COMPARISON 62

5.2.2 INCREASING STRESS RESPONSE AS A FUNCTION OF 63 DECREASING DIMENSIONALITY

5.3 CONDUCTIVE COMPOSITE PHOTO-MECHANICAL RESPONSE 67

5.4 ACTUATION EFFICIENCY AS A FUNCTION OF 69

DIMENSIONALITY

5.5 CONCLUSIONS 71

6 STIMULI-RESPONSIVE TRANSFORMATION IN CARBON 76

NANOTUBE/EXPANDING MICROSPHERE-POLYMER COMPOSITES

6.1 INTRODUCTION 76

6.2 STIMULI RESPONSIVE TRANSFORMATION 78

6.2.1 BINARY MECHANICAL PROPERTIES 81

6.2.2 ELECTRICAL CHANGES DUE TO SHIFTING NANOTUBE 86 JUNCTIONS

6.3 ELECTRICAL APPLICATIONS 91

6.4 CONCLUSIONS 92

7 GRAPHENE/ELASTOMER COMPOSITE-BASED PHOTO THERMAL 98 NANOPOSITIONERS

$\begin{array}{ll}7.1 \text { INTRODUCTION } & 98\end{array}$

7.2 RESULTS 99

7.2.1 PRINCIPLE OF GNP/ELASTOMER PHOTO-THERMAL 99 ACTUATION

7.2.2 PHOTO-THERMAL NANOPOSITIONING SYSTEM 100

7.2.3 GNP/PDMS ACTUATOR FABRICATION AND 101 CHARACTERIZATION

7.2.4 NANOPOSITIONER SYSTEM INTEGRATTION 104

7.2.5 KINETICS OF PHOTO-THERMAL NANOPOSITIONERS 107 
7.2.6 USEFUL DISPLACEMENT VERSUS MAXIMUM DISPLACEMENT

7.2.7 VACUUM DEPENDENCY OF PHOTO-THERMAL NANOPOSITIONER

7.2.8 ACCURACY AND RESOLUTION

7.2.9 ENERGY CONVERSION EFFICIENCY

7.2.10 LONG-TERM CYCLING AND COMPOSITE ACTUATOR DEGRADATION RESPONSE

7.3 CONCLUSIONS

8 SPRING-MASS PHOTOMECHANICAL MODELING

8.1 BACKGROUND

8.2 MODEL SETUP

8.2.1 EQUATIONS OF MOTION FOR INITIAL (NONILLUMINATED) SYSTEM

8.2.2 EQUATIONS OF MOTION FOR RESPONSE TO IR ILLUMINATION

8.3 RESPONSE TO ENVIROMENTAL TEMPERATURE CHANGE

8.5 CONCLUSIONS AND FUTURE DIRECTIONS 138

9 CONCLUSIONS AND FUTURE RESEARCH 145

9.1 CONCLUSIONS 145

9.2 FUTURE RESEARCH 149

REFERENCES $\quad 152$

APPENDICES 165

CURRICULUM VITAE 188 


\section{LIST OF FIGURES}

PAGE

2.1 Atomic nanostructure detail for various graphitic carbon forms. 13

2.2 Hexagonal and rhombohedral graphite layer stacking arrangements. 15

2.3 Carbon nanostructure surface area as a function of graphitic layers. 17

2.4 Relative timeline of actuation technologies. 20

2.5 Coupling of various effects in smart materials/structures. 21

3.1 GNP/PDMS photo-mechanical actuator characterization and testing. 35

3.2 Photo-mechanical responses and actuation kinetics of GNP/PDMS 37 composites.

3.3 Comparison of increasing photo-mechanically induced stress change in GNP/PDMS composites.

3.4 Comparison of photo-mechanically induced stress change in $2 \mathrm{wt} \%$ composites of various carbon forms.

3.5 GNP/PDMS actuator characteristics.

3.6 Steady-state temperature measurements.

4.1 SEM and Raman carbon characterization.

4.2 Composite fabrication and test setup. $\quad 52$

4.3 SLG/PDMS photo-mechanical stress responses and Young's modulus. $\quad 54$

4.4 NIR-induced stress and efficiency comparison between SLG and 56 GNP-based composites.

5.1 Carbon additive characterization. 
5.2 Carbon/PDMS photo-mechanical actuator fabrication and testing. 62

5.3 Stress response and long-term characterization for SLG/PDMS. 63

5.4 Comparison of increasing photo-mechanically induced stress change with 64 decreasing carbon dimensionality.

5.5 Photo-induced changes in conductive composites. 68

5.6 Dimensional dependence of photo-mechanical response. $\quad 70$

$\begin{array}{lll}6.1 & \text { Expanding microsphere overview. } & 78\end{array}$

6.2 Plot of resistivity $(\rho)$ versus SWNT $w t \%$ loading. $\quad 79$

6.3 Effects of microsphere expansion. $\quad 80$

6.4 Expansion and density overview. $\quad 82$

6.5 Volume and dimensional expansion plugs. 83

$\begin{array}{lll}6.6 & \text { Stress-strain overview. } & 85\end{array}$

6.7 SWNTs imparting mechanical strength in expanded composites. 86

6.8 Electrical overview and response regions 1-3. 88

6.9 Relative IR-induced expansion in various $w t \%$ SWNT composites. 90

6.10 Embedded circuit fabrication sequence. 92

6.11 Assorted applications. $\quad 94$

6.12 Contracting skin fabrication sequence. 95

6.13 Application example showing use of patterned TEM regions to serve as 97 structural component.

$\begin{array}{lll}7.1 & \text { Photo-thermal overview. } & 101\end{array}$

$\begin{array}{lll}\text { 7.2 GNP/PDMS composite characterization. } & 103\end{array}$

$\begin{array}{lll}7.3 & \text { Nanopositioner overview. } & 105\end{array}$

$\begin{array}{lll}7.4 & \text { PWM signals. } & 106\end{array}$

7.5 Kinetics curve fitting and individual diode test details. 108 
7.6 Positioning diode response characterization. 112

7.7 Stage response and stability in automatic control mode to position 115 requests for both the $x$ and $y$ axes ( $1 \mathrm{wt} \%$ GNP composite).

7.8 Actual stage response under optical microscope. 117

7.9 Photo-thermal efficiency and long-term degradation. 119

$\begin{array}{lll}\text { 8.1 Spring constant relationships. } & 126\end{array}$

$\begin{array}{lll}8.2 & \text { Initial system model. } & 127\end{array}$

$\begin{array}{lll}\text { 8.3 System model as multiple springs in series. } & 128\end{array}$

8.4 System response to IR illumination. 132

8.5 System response to IR illumination. 134

8.6 Illumination area to contraction relationships. 136

8.7 Multiple stages on a single actuation diagram. 138

8.8 Actuator array fabrication sequence. 141

8.9 Optical mask positioning and array response detail. 142

$\begin{array}{lll}8.10 & \text { Array control detail. } & 143\end{array}$ 


\section{CHAPTER 1 \\ INTRODUCTION}

Motivated by an ever-increasing need for application-specific, small-scale, and custom-made actuation technologies, the past century has witnessed an explosion in this area of scientific research. Disciplines ranging from metallurgy ${ }^{1}$ to chemistry ${ }^{2}$, mechanical engineering ${ }^{3}$, and chemical engineering ${ }^{4}$ have all made major strides in development of novel materials capable of small-scale actuation. Corresponding advancements in fields such as electrical engineering ${ }^{5}$, optics $^{6}$, and physics ${ }^{7}$ have provided a wealth of innovative means to power such actuators. Advancements in the fields of micro and nanotechnology ${ }^{8}$, providing both new materials ${ }^{9-11}$ as well as fabrication processes ${ }^{12-14}$, however, have enabled actuation and positioning devices to be created not only with unprecedentedly high accuracies and resolutions ${ }^{15}$, but also on size scales scarcely imaginable just decades ago ${ }^{16-18}$. Biomedical applications provide perhaps some of the most potent examples of this ongoing micro- and nano-actuator revolution. For example, micro- and nano-actuation technologies are being applied in targeted drug delivery systems ${ }^{19-21}$, employed as implantable devices to augment failing organs ${ }^{22}$, and used for capture of circulating tumor cells towards early metastatic cancer detection ${ }^{23}$.

Moving beyond biomedical applications, recent years have also seen impacts of nanopositioning systems in areas as diverse as scanning probe microscopy ${ }^{24,25}$, metrology ${ }^{26,27}$, advanced manufacturing ${ }^{27,28}$, biological processes ${ }^{29}$, materials science ${ }^{30}$, 
high density storage/memory ${ }^{31,32}$, and precision machining ${ }^{33,34}$. While the most common nanopositioning systems are capable of positioning below $\sim 1 \mathrm{~nm}$ and employ piezoactuated flexure-based systems with feedback control ${ }^{35,36}$, nanopositioning systems based on ferroelectrics ${ }^{37}$, shape memory alloys ${ }^{38}$, electrostrictive materials ${ }^{39}$, dielectric elastomers ${ }^{40,41}$, liquid crystal elastomers ${ }^{42}$, and conducting polymers ${ }^{43}$ have also been demonstrated. As each material offers a unique set of properties, incorporation into positioning systems result in a combination of benefits and compromises. For example, conducting polymer actuators are quite interesting as they offer high strains at low operating voltages ${ }^{44}$ and can exhibit performance characteristics equaling or exceeding those of natural muscle ${ }^{45}$. Disadvantages such as long time constants ${ }^{46}$ and premature breakdown, however, hinder some practical applications ${ }^{47}$. Likewise, piezoelectric systems, the current workhorse of nanopositioning systems, are capable of sub-nanometer positioning ${ }^{35,36}$; but low maximum strains require complex actuator stacks in order to realize larger displacements ${ }^{48}$. While all of these various actuation technologies are impressive, introduction of new types of materials that provide even more innovative capabilities are needed to expand the scope of next-generation high-performance and low-cost nano-positioning/nano-manipulation applications.

Recent additions to the existing family of actuation materials include nanostructures such as carbon nanotubes $(\mathrm{CNTs})^{49}$ and porous metallic nanoparticles ${ }^{50}$ as they exhibit large stresses and strains from low voltage electro-mechanical actuation. Similarly, both single- (SWNT) and multi-wall CNT (MWNT) composites have been reported to undergo light-driven, or photo-mechanical, actuation ${ }^{51,52}$. As such, CNTs, as well as other forms of graphitic nanocarbons, present intriguing possibilities for use as 
actuation materials not because of their electro-mechanical properties, but rather because of their photo-mechanical characteristics ${ }^{53-55}$. Compared to electrically/thermally/ionically/phase-transition driven actuators, light-driven actuators have several advantages including wireless/remote actuation, displacement control using infrared (IR) intensity modulation (controlling number of photons), electrical-mechanical decoupling (and therefore low noise), elimination of electrical circuits at the point of use, and massive parallel actuation of device arrays from a single light source. Photomechanical actuators already have demonstrated possible applications encompassing photonic switches ${ }^{56}$, micro-electro-mechanical system (MEMS)-based micro-grippers for object manipulation $^{57,58}$, robotics ${ }^{59}$, plastic motors ${ }^{60}$, and adaptive micro-mirrors ${ }^{61}$. These applications, combined with recent research in CNT/elastomer ${ }^{51,52,62-65}$ and graphene/elastomer photo-actuators ${ }^{53-55}$ have generated renewed excitement in photoactive materials and their application as micro-/nano-optomechanical systems ${ }^{57,58,61}$.

Since graphene's 2004 discovery ${ }^{9}$, atomically thin carbon films have been the subject of intense research and their incredible physical properties well documented, including thermal conductivity ${ }^{66}$, mechanical strength ${ }^{67}$, and quantum hall effect at room temperature ${ }^{68}$. Two-dimensional (2D) graphene crystals have shown unique layer dependent properties, such as saturable absorption ${ }^{69}$, linear monochromatic optical contrasts $^{70}$, and electric field-assisted band gaps for graphene bi-layers ${ }^{71}$. A single graphene layer absorbs $\sim 2.3 \%$ of incident light independent of its wavelength and reflectivity is low $(<0.1 \%)^{72}$. The absorbance of multilayers of graphene at photon energies greater than $\sim 0.5 \mathrm{eV}$ should be additive $\mathrm{e}^{73}$. Photo-thermal mechanisms suggest that optically induced thermal gradients will result in a linear increase in photo-current 
response with increasing laser intensity ${ }^{74}$. Because of these properties, graphene presents an intriguing material in high performance polymer composites. For example, graphene/polymer composites are gaining attention for use in light-weight applications including sports equipment, wind turbines, aircraft composites, and medical implants ${ }^{75}$.

While numerous articles report on graphene-based composites, most of these actually used graphene-like sheets derived from graphite oxide (GO) or graphite intercalation compounds (GICs). GO- and GIC-derived fillers in polymer matrices can exhibit high electrical conductivities ${ }^{76}$, high Young's moduli ${ }^{77}$, and easily be functionalized to compliment host polymer properties $^{78}$. While GO- and GIC-derived filler materials report electrical conductivities better than those reported for nano-clays ${ }^{79}$, however, these are often lower than values reported for single-wall CNT fillers ${ }^{80}$. Secondly, graphene nanoplatelets (GNPs) and graphene nanoribbons (GNRs) have properties that are dependent on number of layers, such as saturable absorption ${ }^{69}$, linear monochromatic optical contrasts ${ }^{70}$, and electric field-assisted band gaps ${ }^{71}$, which are not exhibited in GO- and GIC-derived materials. These interesting properties call for a thorough investigation of graphitic nanocarbon-based composite photo-mechanical actuators and their potential applications.

Therefore, goals of this dissertation are: (1) develop carbon nanostructure/elastomer composites with ability to undergo photo-mechanical actuation; (2) investigate the mechanisms responsible for photo-mechanical actuation; (3) investigate effects of number of graphitic layers in the dispersed carbon nanostructures on realized photo-mechanical actuation and efficiency; (4) investigate photo-mechanical stress and efficiency responses according to carbon nanostructure dimensional state; (5) 
develop fabrication processes to disperse the various types of carbon nanostructures within the elastomeric matrix; (6) develop a simple spring-mass mathematical model of photo-mechanical actuation and compare this model to experimental data; and (7) demonstrate practical device applications of photo-mechanical actuation in both the micro and macroscopic realms. To analyze and present these goals, this dissertation is organized into the following chapters:

- Chapter 2 provides background into the various types of carbon nanostructures used and evaluates existing micro- and nano-actuation technologies.

- Chapter 3 details for the first time, intrinsic photo-mechanical actuation of GNP-based polymer composites. These photo-mechanical actuators showed reversible and repeatable light-induced elastic expansion and contraction.

- Chapter 4 compares photo-mechanical responses between GNP and single layer graphene (SLG) and reports on composite stress responses, elastic moduli, and efficiencies, which were observed to depend on the number of graphene layers in the 2D crystal.

- Chapter 5 provides further investigation into photo-mechanical actuation responses in various types of carbon nanostructure/polydimethylsiloxane (PDMS) composites. Utilizing an evaporative mixing fabrication method, carbon/polymer composites were made with one-dimensional (1D) multi-wall carbon nanotubes, 2D single-layer graphene, two and a half-dimensional (2.5D) GNPs, and three-dimensional (3D) highly ordered pyrolytic graphite (HOPG). Dimensionally dependent photo-mechanical stress responses and efficiency in response to near-infrared (NIR) illumination are reported. Furthermore, photo-mechanical actuation kinetics and their 
relationship to the percolation threshold are presented, showing a shift from localized response (nonconductive composite) to global response (conductive composite). Following this thorough characterization of nanocarbon/elastomer composites' photomechanical properties, research shifted to practical demonstration of micro- and macro-scale photo-mechanical actuators.

- Chapter 6 presents development of a ternary composite system based on carbon nanotubes and thermally expanding microspheres dispersed within an elastomer matrix that exhibits ability to visibly and macroscopically unidirectional transform properties (strength, conductivity, density, and volume) in response to IR stimuli.

- Chapter 7 demonstrates application of photo-mechanical actuation in a two-axis submicron resolution translation stage. The positioner is feedback-controlled by differential illumination of opposing composite elastomer actuators, which automatically stabilizes the stage against thermal drift as well as random thermalinduced position fluctuations (up to the bandwidth of the feedback and position sensor).

- Chapter 8 presents insights into the physics behind photo-mechanical actuation principles by presenting derivation of a spring-mass model for composite actuation and a comparison of this model to experimental data. Additionally, application of the model to both single- and multi-stage positioning systems are discussed.

- Finally, Chapter 9 concludes the dissertation, and provides several suggestions regarding future direction for continued work on photo-mechanical nanocarbon-based composite actuators. 
The following list summarizes a variety of original contributions to the field of photo-mechanical actuation that resulted from this research:

(1) Demonstration of photo-mechanical effects in graphene nanoplatelets-based polymer composites for the first time, including an extraordinary optical-tomechanical energy conversion factor of 7-9 MPa/W.

(2) Development of new dispersion method for difficult to separate nanocarbons into polymer matrixes (evaporative mixing).

(3) Direct correlation between increasing photo-mechanical stress and increasing efficiency with reducing dimensionality of binary carbon/elastomer composites.

(4) Discovery that actuation and relaxation kinetics in carbon/elastomer composites are not a function of dimensionality, but rather related to the percolation threshold of carbon nanostructure additive in the polymer. Establishing a connective network was found to change photo-mechanical stress in the composite from a local (fast) to global (slow) response.

(5) For composites greater than the percolation threshold, showing that photo-conductive response was inversely proportional to applied pre-strain, demonstrating electrical-mechanical coupling.

(6) Development of a ternary expanding microsphere/CNT-based stimuli-responsive composite with ability to unidirectionally transform physical dimensions ( $>500 \%$ volume increase), elastic modulus ( $>675 \%$ increase), density ( $>80 \%$ reduction), tunable electrical response, and overall elastic to plastic-like transformation.

(7) Variety of demonstrations of how ternary expanding microsphere/CNT composites could be used (a) to design circuits with controllable resistance, (b) to fabricate 
actuator elements which enable both contractive as well as expansion actuation, and (c) in conjunction with multi-layer patterning methods for on-demand structural components.

(8) First practical demonstration of a two-axis sub-micron translation stage. Utilizing dynamic modulation of multiple independent infrared sources placed along the actuators which enabled differential control over polymeric chain stretching/contraction.

(9) Development of a spring-mass model of photo-mechanical actuation. Simple carbon/elastomer composite actuators are modeled as a series of springs, each with its own independently controllable (via IR illumination) spring constant.

This work resulted in several journal publications, including:

[1] Loomis, J., Xu, P., Fan, X., Fletcher, M., Cohn, R. W. \& Panchapakesan, B. Graphene/elastomer composite-based photo-thermal nanopositioners. Nature Scientific Reports. (in press).

[2] Loomis, J., Xu, P. \& Panchapakesan, B. Stimuli-responsive transformation in carbon nanotube/expanding microsphere-polymer composites. Nanotechnology. 24, 185703 (2013).

[3] Xu, P., Loomis, J., Bradshaw, R. D. \& Panchapakesan, B. Load transfer and mechanical properties of chemically reduced graphene reinforcements in polymer composites. Nanotechnology. 23, 505713 (2012).

[4] Xu, P., Loomis, J., King, B. \& Panchapakesan, B. Synergy among binary (MWNT, SLG) nano-carbons in polymer nano-composites: a Raman study. Nanotechnology. 23, 315706 (2012).

[5] Xu, P., Loomis, J. \& Panchapakesan, B. Photo-thermal polymerization of nanotube/polymer composites: Effects of load transfer and mechanical strength. Applied Physics Letters. 100, 131907 (2012). 
[6] Loomis, J. \& Panchapakesan, B. Dimensional dependence of photomechanical response in carbon nanostructure composites: a case for carbon-based mixeddimensional systems. Nanotechnology. 23, 215501 (2012).

[7] Loomis, J., King, B. \& Panchapakesan, B. Layer dependent mechanical responses of graphene composites to near-infrared light. Applied Physics Letters. 100, 073108 (2012).

[8] Loomis, J., et al. Graphene nanoplatelet based photomechanical actuators. Nanotechnology. 23, 045501 (2012).

Additionally, this work generated several conference papers, including:

[1] Loomis, J. \& Panchapakesan, B. Carbon based dimensional composites. Tech Connect World 2012 Proceedings. 1, 286-289 (2012).

[2] Loomis, J., King, B. \& Panchapakesan, B. Near-infrared light induced mechanical responses in single-to-few layer graphene. Tech Connect World 2012 Proceedings. 1, 294-297 (2012).

[3] Loomis, J., et al. Photomechanical response in graphene nanoplatelet composites. Tech Connect World 2012 Proceedings. 1, 290-293 (2012).

Finally, this work resulted in two provisional patents, including:

[1] Loomis, J., Cohn, R. W., \& Panchapakesan, B. Graphene/elastomer compositebased photo-thermal nanopositioners, in progress. (2013).

[2] Loomis, J., \& Panchapakesan, B. Stimuli-responsive polymer composites, United States U. S. Provisional Patent Application Serial No. 61/762,658. (2013). 


\section{CHAPTER 2 \\ BACKGROUND REVIEW}

\subsection{INTRODUCTION}

Recently, a new type of photo-mechanical actuator based on graphitic carbon forms mixed in polymeric materials has demonstrated extraordinary mechanical response in SLG/polymer ${ }^{53}$, GNP/polymer ${ }^{54}$, and CNT/polymer composites ${ }^{51,52,57,58,63,65,81}$, thus revilitializing interest in photo-mechanical actuation. All of these new graphitic carbon forms have been widely synthesized, with their structure, electronic, and optical properties characterized ${ }^{9,10,82-84}$. Photo-mechanical actuators have already shown possible

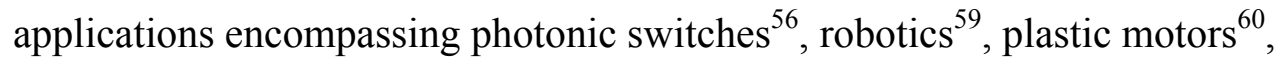

micro-opto-mechanical grippers ${ }^{57,58}$, and adaptive micro-mirrors ${ }^{61}$. While past research has extensively studied physical properties of synthetic graphitic nanostructures and their composites, none has focused on exploiting dimensional state $(1 \mathrm{D}, 2 \mathrm{D}$, or $3 \mathrm{D})$ of the carbon nanostructure in the composite to affect an overall mechanical response. This expanded focus could enable not only new types of mechanical actuators based on carbon nanostructure dimensionality, but also a variety of opto-electronic devices based on mixed-carbon nanostructures.

Of these devices, the most interesting are those based on carbon nanostructure additives dispersed within PDMS, a common silicone elastomer. Carbon/PDMS composite photo-mechanical actuators have demonstrated both expansive as well as 
contractive mechanical responses. The extraordinary ability to both expand (at low applied pre-strains) and contract (at high applied pre-strains) is due to a phenomenon known as rubber elasticity. Discovered by Gough in 1805 and confirmed by Joule in $1859^{85}$, they observed that rubber under a constant strain reversibly contracts upon heating. Homogeneously dispersing carbon nanostructure additives within the polymer provides an efficient mechanism for thermal energy transduction from photons to polymeric chains; therefore, structures fabricated from carbon/PDMS composites can be selectively pre-strained to achieve a desired response type. For example, at low applied pre-strains $(<\sim 10 \%)$, the load on the composites is less than the thermoelastic inversion point; therefore, composite samples exhibit expansion upon NIR illumination. At high applied pre-strains ( $>\sim 15 \%)$, however, NIR illumination and subsequent heating invokes a contractive response. This effect, combined with the ability to tailor polymeric properties through incorporation of carbon nanostructure additives, makes photomechanical actuation in nanocarbon/polymer composites both interesting and potentially a new vehicle for exploiting rubber elasticity in actuator applications.

\subsection{CARBON NANOSTRUCTURES}

Carbon, the fourth most abundant element in the universe ${ }^{86}$, plays a vital role not only cosmically in interstellar evolution ${ }^{87}$, but also terrestrially both as a fundamental building block of life ${ }^{88}$ as well as in cutting edge man-made devices such as sensors and actuators ${ }^{89,90}$. Carbon exists in various forms, or allotropes, with the best known being graphite, diamond, and amorphous carbon ${ }^{91}$. Allotropes have a wide variety of unique properties, for example, hardness, which ranges from 10 for diamond ${ }^{92}$ (Mohs mineral hardness scale) to just $\sim 1.5$ for graphite ${ }^{93}$. For thousands of years, these two forms were 
the only known carbon allotropes ${ }^{91}$. Recently, in addition to the discovery of altogether different allotropes, synthetic carbon forms have expanded the already known families. For example, synthetic graphitic carbon forms such as buckyballs ${ }^{94}$, nanotubes $^{10}$, and graphene ${ }^{9}$ have emerged as some of the most widely researched materials during the past decade due to interesting physical properties, which include extraordinary mechanical strengths $^{95}$, high electron mobilities ${ }^{96}$, room temperature quantum Hall effect ${ }^{97}$, and optical absorption $^{72,98-100}$.

Graphitic carbon forms are comprised of hexagonal rings of carbon atoms joined by hybridized $\mathrm{sp}^{2}$ bonds ${ }^{101}$. SWNTs are hollow 1D tubes of carbon atoms with nanometer diameters and lengths in the micron range, while MWNTs can be thought of as two or more concentric SWNTs [Figure 2.1(a) $]^{10}$. SLG is an atomically flat 2D sheet of carbon atoms arranged in a honeycomb lattice [Figure 2.1(b) $]^{9}$. Few-layer graphene (FLG), or GNPs, are comprised of $\sim 3-5$ atomic layers of graphene ${ }^{102}$. Due to this layering, they can be thought of as 2.5D nanostructures [Figure 2.1(c)]. Finally, anything greater than $\sim 10$ sheets of carbon atoms is considered a 3D thin graphite film [Figure 2.1(d) ${ }^{103}$.

While all of these structures are chemically identical, their material properties can be markedly different - one of the underlying reasons for this investigation into carbon nanostructure-based composites. Far from being the only incentive, however, a host of other motivations also exist, including: (1) Carbon nanostructures are efficient at light absorption and subsequent energy transduction to polymeric chains, thus enabling resulting composites to undergo photo-mechanical actuation. (2) Carbon nanostructures introduce improved mechanical properties to the PDMS matrix. Hence, evaluation of differences in resulting photo-mechanical properties between carbon forms will allow for 


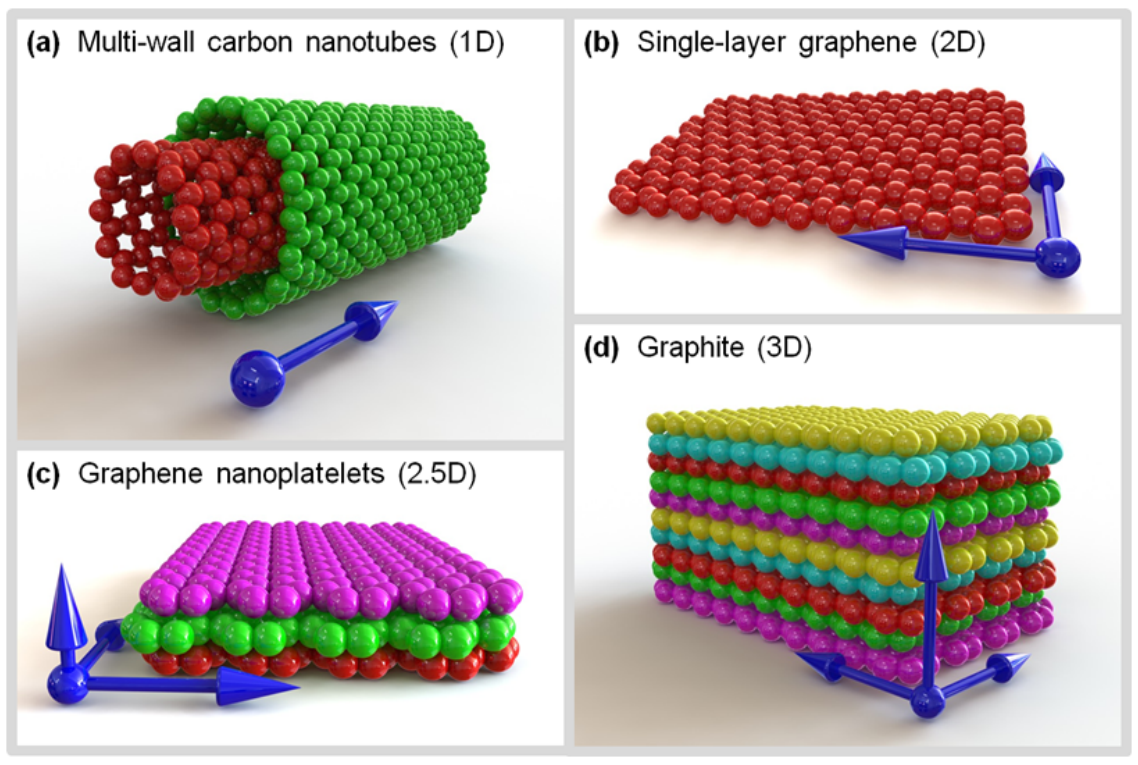

Figure 2.1. Atomic nanostructure detail for various graphitic carbon forms, with blue coordinate system arrows indicating degree of dimensionality, and different colors used to illustrate different layers of carbon atoms: (a) MWNT, a 1D material; (b) SLG, a 2D material; (c) GNP, a 2.5D material; and (d) graphite, a 3D material.

creation of application-specific composites. (3) Conductive properties of carbon nanostructures can be used to introduce sensing properties into the composites, resulting in the ability to sense and actuate in a single solid state material. (4) Use of nanostructured materials in general is still in its infancy, and therefore of enormous scientific interest ${ }^{104}$. (5) Perhaps most importantly, the $\mathrm{sp}^{2}$ bonding configuration results in interesting material geometries well suited for creating different dimensional forms, making carbon-based composite research interesting from both science and application aspects.

The plethora of multiple graphitic carbon forms is due to the $\mathrm{sp}^{2}$ carbon bonds found in graphite, carbon nanotubes, and graphene. The importance of this bond on 
resulting material properties can be highlighted by comparing graphite to diamond (another carbon allotrope). While diamond contains tetrahedral $\mathrm{sp}^{3}$ carbon bonds resulting in a strong overall isotropic $3 \mathrm{D}$ structure, graphite has triangular $\mathrm{sp}^{2}$ bonds, which in turn form discrete carbon layers (graphene layers) within the macroscopic material $^{105}$. Individual layers within graphite are stacked at $\sim 3.354 \AA$ spacing, contain hexagonal arranged carbon atoms at symmetrical $\sim 1.421 \AA$ and $120^{\circ}$ atom-to-atom spacing and bond angles respectively ${ }^{106}$, and are held together through weak van der Waals forces (lack of covalent bonds between layers helps make graphite an ideal material in applications such as lubricants) ${ }^{105,107}$. Interlayer forces in graphite are estimated to range between $0.97-5.0 \mathrm{kcal} / \mathrm{mole}^{106}$, resulting in the ability to easily cleave graphite along its basal planes. Graphite layers can stack in either hexagonal (A-B-A-B) or rhombohedral (A-B-C-A-B) arrangements (as Figure 2.2 shows), with hexagonal arrangements being much more prevalent ${ }^{107}$. This layering further results in highly anisotropic material properties in graphite, such as high electrical/thermal conductance in plane (within the individual carbon layers) but poor out of plane (between layers). For example, thermal conductivity within a graphite layer is $\sim 20 \mathrm{~W} /{ }^{\circ} \mathrm{C} \cdot \mathrm{K}$, about 200 times higher than conductivity between layers ${ }^{106}$. Additionally, monocrystalline graphite films have been shown to withstand currents $>10^{8} \mathrm{~A} / \mathrm{cm}^{2}{ }^{108}$. Finally, chemical reactants can reside in-between graphitic carbon layers and form compounds known as GICs, which in turn can be exfoliated upon application of heat ${ }^{105}$.

A majority of research into fundamental graphite properties has been conducted using either naturally occurring single-crystal graphite or pyrolytic graphite $(\mathrm{PG})^{105}$. Problems with these forms, however, include both small size of naturally occurring 

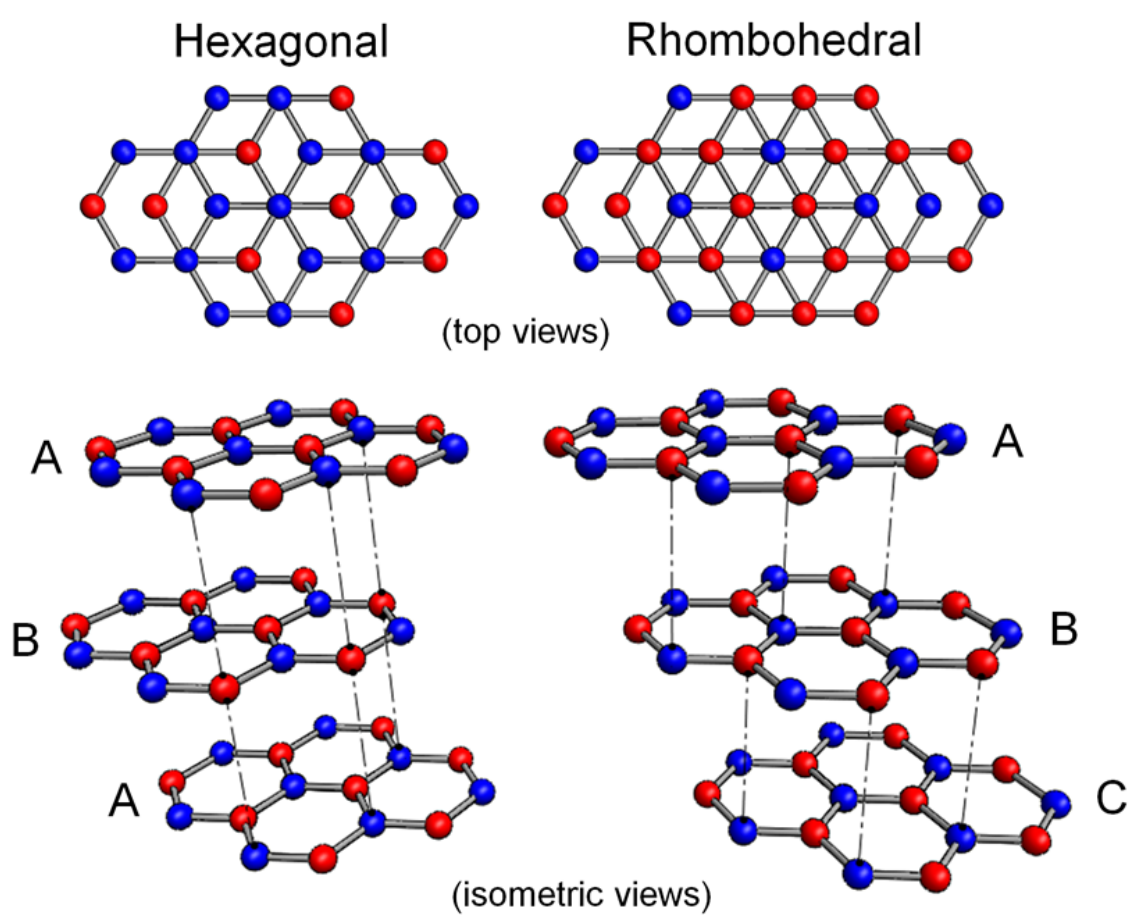

Figure 2.2. Hexagonal and rhombohedral graphite layer stacking arrangements ${ }^{109}$.

single-crystal graphite, as well as the presence of crystalline defects and elemental impurities; thus, HOPG is used in most current graphite research ${ }^{110}$. HOPG is a polycrystalline material containing highly ordered stacked graphene sheets, with dimensions of $\sim 1-10 \mu \mathrm{m}$ (parallel to the basal plane) by $>0.1 \mu \mathrm{m}$ (perpendicular to the basal plain ${ }^{111}$. HOPG is produced through heat treating and annealing of pyrolytic carbon (exact material properties of HOPG are dependent on preparation methods and conditions ${ }^{112}$ ) and results in material properties such as electronic, thermal, and 1-to-mechanical energy conversion of $\sim 7 \mathrm{MPa} / \mathrm{W} \perp$ !! $\mathrm{HYPE}^{110}$, thus making it an ideal for research applications. Interestingly, as the number of graphitic layers is reduced, the resulting material (known as FLG or GNPs) has hybrid properties between those of bulk graphite and single-layer graphene ${ }^{113}$. As with graphite, stacking geometry of stacking and number of layers in GNP strongly affects electron transport properties ${ }^{113}$. One 
method of producing graphene nanoplatelets is through a process known as a split plasma technique, which opposed to wet chemical methods of nanoplatelet production, is not thought to degrade the structural integrity and mechanical strength of the nanostructures $^{114}$.

Regarding use of carbon nanostructures in composites, for a given filler material, macroscopic composite material properties depend on the carbon-polymer interface ${ }^{115}$. With all other factors being equal, as the number of graphitic layers in the carbon nanostructure decreases, effectiveness as a reinforcement material in a polymer composite should increase. The fewer graphitic layers per nanostructure, the more carbon surface area is available for bonding with the polymer matrix - giving rise to increased strength, more efficient energy transduction, and higher elastic modulus. Figure 2.3(a) shows that for a hypothetical amount of graphitic carbon nanostructure, the drastic decrease in exposed carbon surface area available for bonding with the matrix as the number of stacked graphene layers increases. Figure 2.3(b) shows the same relationship but with a $\log -\log$ scale, which more clearly illustrates the 2D, 2.5D, and 3D regions. As number of graphitic layers is reduced, eventually only a single 2D atomic layer of carbon atoms will remain - yielding single layer graphene. The SLG used in this dissertation was obtained by chemical reduction of graphene oxide (rGO). Reduction is a process that serves to remove the oxygen-containing groups and decrease the number of crystalline defects in the graphene oxide ${ }^{116,117}$. While material property improvements are realized, however, it is not possible to remove all the residual functional groups and defects; thus, reduction results in a material with "graphene-like" properties rather than true or “pristine" SLG ${ }^{116}$. As opposed to chemical reduction, production of pristine SLG can be 
accomplished through mechanical exfoliation of HOPG or via chemical vapor deposition (CVD); both these methods result in pristine graphene sheets with near perfect atomic structures, and excellent thermal, mechanical, and electrical properties ${ }^{116}$. While mechanically exfoliated or CVD grown graphene is preferred, high costs and low yields limit practical applications. Alternatively, the low-cost, simple reduction method makes SLG obtained from rGO ideal for large-scale usage, such as in photo-mechanical composites presented in this dissertation.
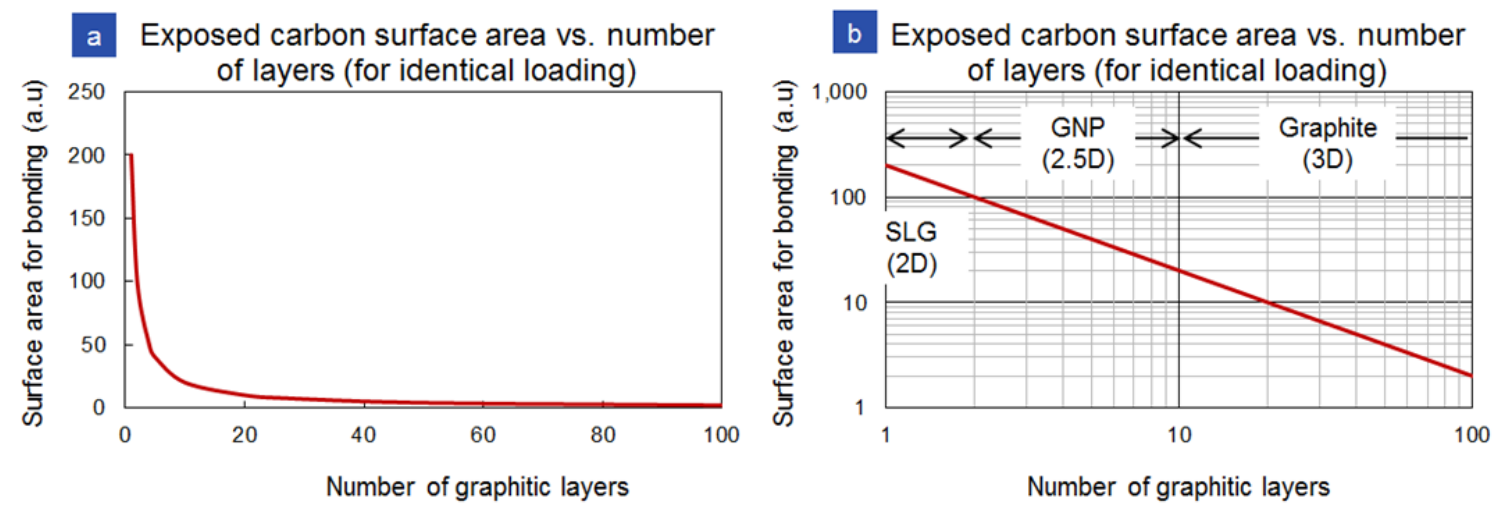

Figure 2.3. Carbon nanostructure surface area as a function of graphitic layers. (a) Plot shows decreasing surface area as number of graphitic layers increases. (b) Log-log scale enables clear distinction of SLG (2D), GNP (2.5D), and graphite (3D) regions.

While a variety of studies have been conducted into mechanical and electrical properties of SLG and GNPs, research into optical and opto-electronic properties is less mature $^{117}$. The advancement of research has been complicated somewhat by demonstration that optical properties of zero-dimensional (0D, buckyballs) and 1D (CNTs) carbon nanostructures do not necessarily predict those of $2 \mathrm{D}$ and $2.5 \mathrm{D}$ carbons ${ }^{117}$. Interestingly, and as opposed to most phenomena in condensed matter physics, graphene opacity can be described solely by using fundamental constants - and 
is not dependent on material properties ${ }^{118}$. In IR-to-visible wavelengths, a single graphene layer absorbs $\pi \alpha$, or about $2.3 \%$ of incident light, and reflectivity is low $(<0.1 \%)^{72}$. The variable $\alpha$ represents the fine structure constant and is equal to $e^{2} / \hbar c$ (where $e$ is elementary charge, $\hbar$ is reduced Plank constant, and $c$ is speed of light) ${ }^{118}$. The relatively large (for a single atomic layer) absorption of graphene is due to two unique properties - namely, the 2D massless fermions and conical band structure ${ }^{119}$. Furthermore, and of importance when modeling GNP-based composites, is that the absorbance of multilayers of graphene at photon energies greater than $\sim 0.5 \mathrm{eV}$ should be additive $^{73,118}$. Less than $\sim 0.5 \mathrm{eV}$, universal absorption characteristics do not apply ${ }^{118}$. Photo-thermal mechanisms suggest that optically-induced thermal gradients will result in a linear increase in photo-current response with increasing laser intensity ${ }^{74}$, however, saturable absorption limits maximum useable IR illumination intensity. For example, with photon excitations of $<1.55 \mathrm{eV}$ (work presented in this dissertation uses $\sim 1.45 \mathrm{eV}$ ), the maximum possible electron density is $\sim 5.8 \times 10^{5} / \mu^{2}{ }^{2119}$. Once this limit is reached, the graphene layer will have $100 \%$ transmittance to additional incoming photons ${ }^{119}$. This phenomena illustrates ability to tailor photo-mechanical response in carbon-based composites by either changing the number of layers in the composite, or altering IR illumination intensity, thus making carbon-based graphitic nanostructures an ideal material for studying photo-mechanical actuation in polymer-based composites.

\subsection{MICRO/NANOACTUATOR TECHNOLOGIES}

Actuation materials and technologies perform useful work in response to a variety of external stimuli, converting energy (thermal/phase, electrical, magnetic, optical, mechanical/acoustic, and chemical/biological ${ }^{120}$ ) into useable work. Due in part to 
various benefits and drawbacks of each type of technology, there currently exists a vast array of solid-state actuation materials across an ever-increasing spectrum of research applications and commercial devices. Application specific requirements such as (1) force/stroke, (2) lifetime, (3) efficiency, (4) environmental conditions, (5) size/weight restrictions, and (6) response times help narrow the field and dictate specific material selection ${ }^{121}$. While advancement of actuation technologies has been important throughout history, only the last century, which through exploitation of exotic material properties, has witnessed the largest introduction of new solid-state technologies. For visual reference, Figure 2.4 shows some major advancements/milestones in actuation technology and approximate number of years since first demonstration. Implications of improved actuation materials and technologies extend well beyond daily conveniences; for example in biomedical applications, more than 100 million humans have at least one major internal medical device ${ }^{122}$. Concurrently with introduction of new material technologies, improvements in processing and fabrication techniques have resulted in increasing importance and focus on MEMS and nano-electro-mechanical system (NEMS) compatible applications. A corresponding "device size" curve is included in the figure that demonstrates this relationship. As more and more commercial MEMS and NEMS devices come online, actuation technologies that lend themselves preferentially to scaling and application in semiconductor friendly processes hold unique advantages. Therefore, new technologies offering high performance operation, as well as compatibility in batch fabrication processes (enabling low-cost, high scalable manufacturing), will be ideal for use in next generation micro- and nanoactuation systems. 


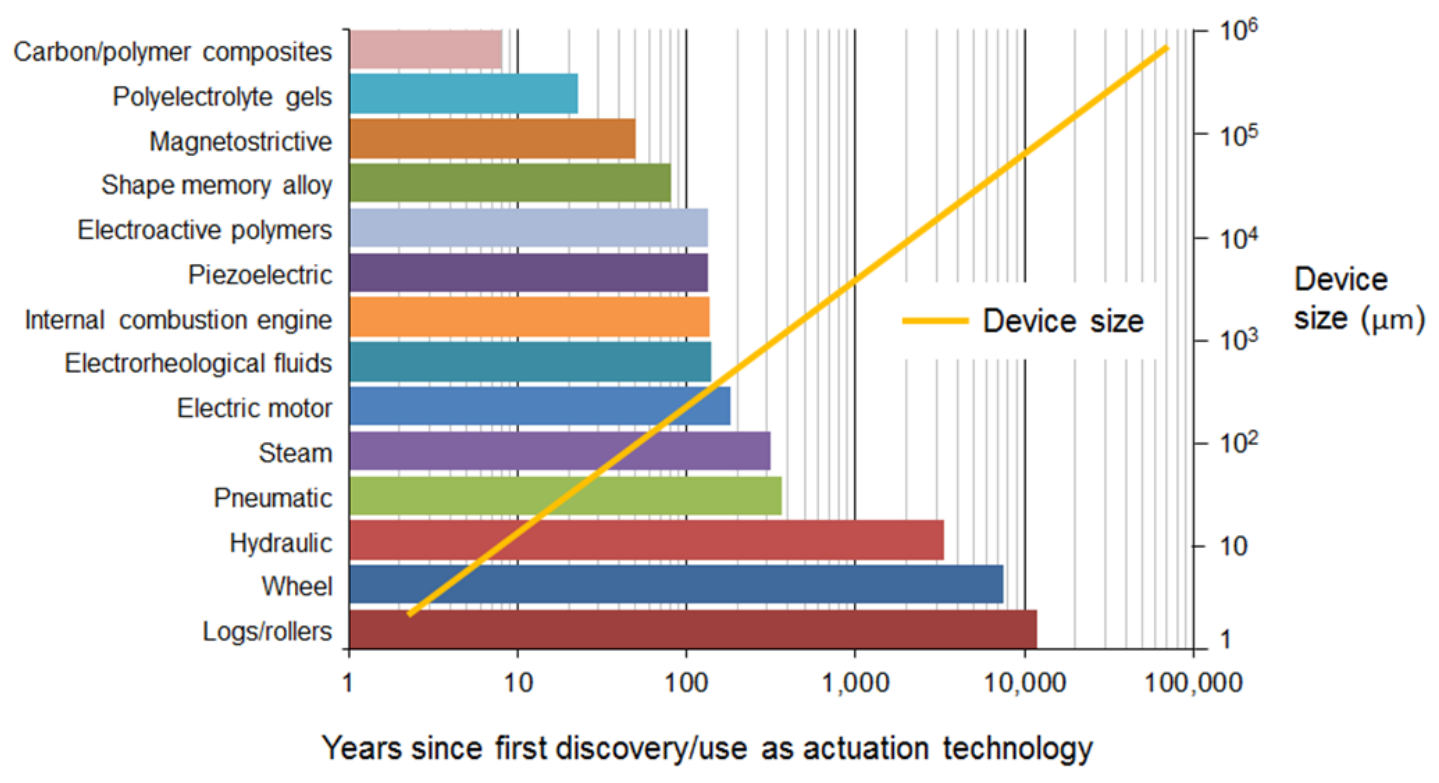

Figure 2.4. Relative timeline of actuation technologies. The bar graphs compares amount of time since some notable actuation technologies/milestones ${ }^{123-132}$.

Additionally, evaluating some of the breakthroughs responsible for new actuation technologies, Figure 2.5 shows coupling between applied input and resulting output as a function of different effects. Some effects are well known, such as the application of heat that results in a corresponding temperature change (which is a function of the material's specific heat). Other effects, such as magnetostriction (change in material shape in response to a magnetic field), however, represent much more recent discoveries and hence possibilities for new applications. Several of these most notable materials are discussed in the following paragraphs, and summarized in Table 2.1. Due to the importance of optically-induced effects, light-driven actuation is covered in a separate sub-section of this chapter. 


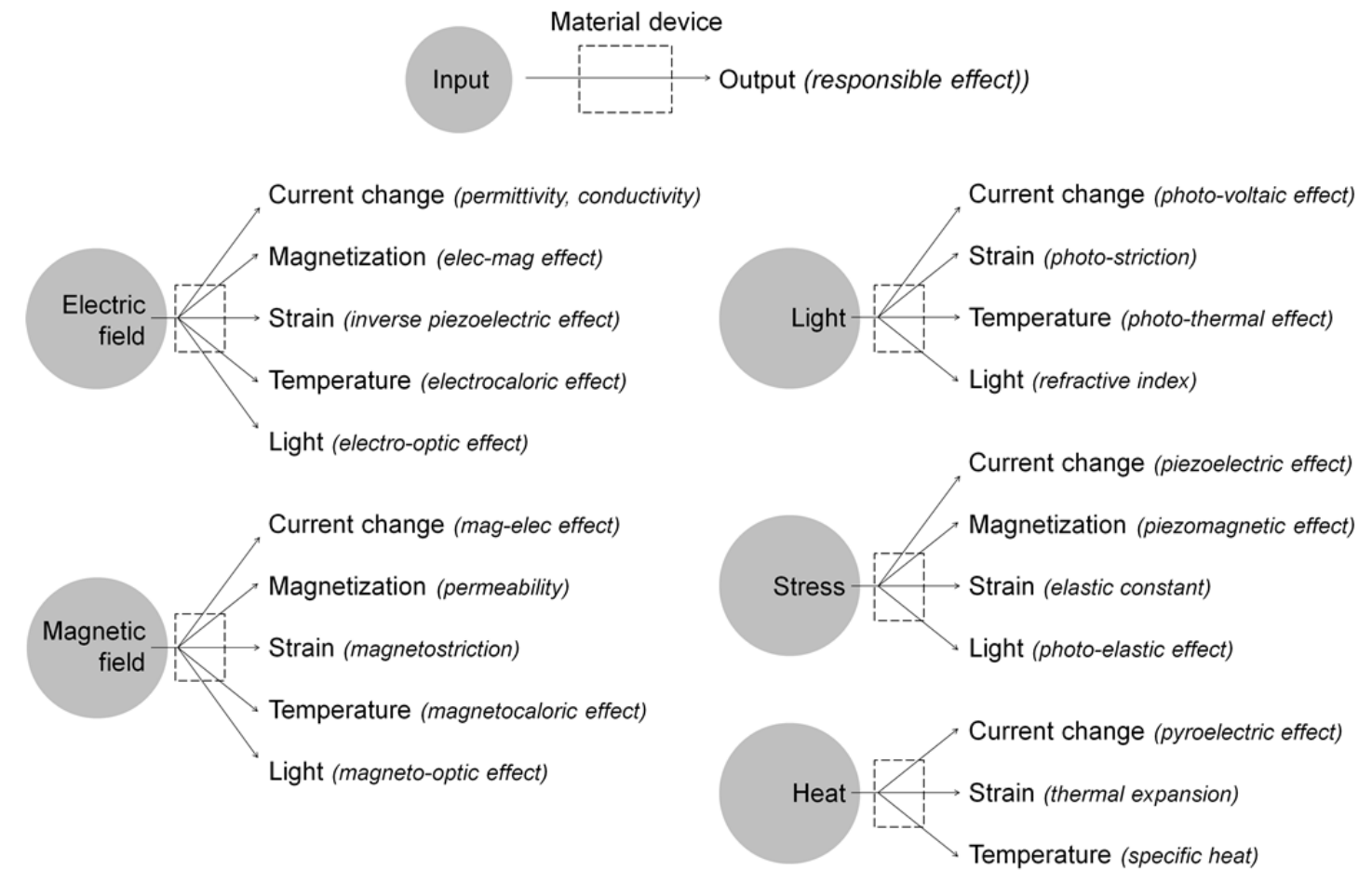

Figure 2.5. Coupling of various effects in smart materials/structures ${ }^{121,129}$.

Piezoelectric. In1880, brothers Pierre and Jacques Curie first discovered that some materials develop surface electrical charges in response to application of physical pressure $^{131}$. Eventually coined the "piezoeffect"(electricity resulting from mechanical pressure) and "return piezoeffect" (elastic deformations due to application of voltage in piezoelectric materials $)^{133}$, significant progress was made throughout the rest of the $19^{\text {th }}$ century ${ }^{134}$. While fruitful, however, it nonetheless took 37 years of research to progress from initial discovery to first practical application - a marine echo depth sounder invented in 1917 by French physicist Paul Langevin ${ }^{134}$. Since then, piezoelectric devices have established themselves as critical components across a swath of industries, with the market for piezoelectric actuators estimated to hit $\$ 12.3$ billion by $2014^{135}$. Some of these applications include as actuators in small-scale insect-inspired robotics ${ }^{136}$; for substrate motion control in atomic force microscopes $(\mathrm{AFM})^{137}$; as transducers in commercial and 
military sonar systems ${ }^{138}$; variety of biomedical uses ranging from implantable sensors ${ }^{139}$ to endoscopic surgery ${ }^{140}$; for material deposition in inkjet printers ${ }^{141}$; to harvest energy from vibration sources ${ }^{142}$; and as accelerometers and resonators in mobile phones and tablets $^{143}$.

The piezoelectric effect occurs in certain crystals that experience change in dimensions due to application of an electric charge ${ }^{144,145}$. A common piezoelectric material is lead-zirconate-titanate (PZT), with the term PZT thus used to refer to piezoelectric materials in general ${ }^{145}$. A PZT material contains electric dipoles arranged in random directions ${ }^{145}$. The natural tendency of these dipoles to an applied electric field, therefore, is to cancel each other out upon application of an electric field, consequently resulting in no net change to the PZT dimensions ${ }^{145}$. In order to enact usable piezoelectric responses from the material, the material must be heated above its Curie temperature and a strong electric field applied in a process called poling ${ }^{145}$. This stage results in alignment of the dipoles that become permanently fixed upon cooling. When the poled ceramic is subjected to a small electric field (at temperatures less than the Curie temperature and electric field small compared to the poling field), the aligned dipoles produce macroscopic expansion along the poling axis and contraction perpendicular to this axis ${ }^{145}$. Piezoeletric materials typically exhibit high response frequency and actuation efficiency, but also have low output strain and work output ${ }^{146}$.

While piezoelectric actuators encompass a host of useful properties, such as subnanometer resolution ${ }^{147}$; fast response time (microsecond time constants); vacuum compatibility; and solid-state actuation, they also present several significant drawbacks and tradeoffs. For example, while piezoelectric actuators can be used at cryogenic 
temperatures ${ }^{148}$, they have relatively low maximum temperatures (must maintain significant margin of safety below the Curie temperature) ${ }^{144}$. In terms of energy, piezoelectric actuators have low power consumption (static operation does not consume power), but a continuous driving voltage is required to maintain displacement. Depending on actuator configuration, large forces can be generated but only at small strains of $\sim 0.1$ to $0.2 \%$ (stack configuration); whereas large displacements are possible but only at low forces (bending configuration). Furthermore, piezoelectric actuators require high driving voltages ${ }^{144}$ while a nonlinear force-stroke results in inability to maintain constant force throughout the entire range of motion ${ }^{149}$ - both are additional considerations for implementation.

Shape memory alloy (SMA). SMA is a generic term given to a class of materials that "remembers" its original shape and can be reverted back to this shape by heating the SMA above a characteristic transformation temperature ${ }^{145}$. SMAs are considered to be one of the major components in emerging smart technologies ${ }^{150}$. Chronologically, the history of shape memory materials began in the early 1930's with the first observation of the pseudoelastic effect Au-Cd alloy ${ }^{132}$, it wasn't until the early 1960's when the shape memory effect was discovered in near-stoichiometric Ti-Ni alloys that the engineering importance began to be realized ${ }^{150,151}$. The majority of SMAs are based on nickel-titanium (Ni-Ti) alloys and referred to as Nitinol, which is a term comprised from these elements and "Naval Ordnance Laboratory", where the discovery was first made ${ }^{152}$.

SMAs' unusual memory behavior is a result of temperature-induced austenite-tomartensite phase transformation ${ }^{152}$. Depending on alloy constituents, SMAs can be inexpensive, fabricated with common metalworking techniques, and have a tunable 


\section{Table 2.1. Comparison of general characteristics between different actuation}

\section{technologies.}

Actuation

Technologies

Shape memory
alloy ${ }^{146,152-154}$
Piezoelectric ${ }^{134,144,145}$
Magnetostrictive $^{144,15}$
Electrorheological $_{\text {fluid }}^{144,145}$

Electroactive

polymers $^{144,156}$

$$
\text { fluid }^{144,145}
$$

Advantages

$\cdot$ High force-to-weight
ratio
$\cdot$ Large strains
$\cdot$ Variable shapes

Variable shapes

- High actuation

frequencies

( $\sim \mathrm{kHz}$ range)

- Low aging effects

- Large forces at small

displacements

- Low aging effects

- Fast response $(\sim \mu s)$

High speed

activation

- Low required current

(but at high voltages)

- Fast response (ms)

- Two orders of

magnitude less active

fluid than an ER

- Less power source

restrictions than ER

- Low sensitivity to

temperature effects

and contamination

- Large category

encompassing
Disadvantages $\quad \begin{gathered}\text { Driving } \\ \text { source }\end{gathered}$

- Actuation loss and fatigue over repetitive

cyclic loading

- Low energy

efficiency

- Slow response times

- Low strains $\left(\sim 10^{-3}\right) \quad \cdot$ Electric $\cdot$ Ink jet printers

- Large hysteresis

field

heads

- AFM stage

- Speakers

- Nonlinear behavior · Magnetic · Vibration

- Low efficiency field control

- Large hysteresis

- Low strains $\left(10^{-4}\right)$

Sonar transducers

- Linear motors

- Limited size scaling · Electric · Hydraulic

capabilities field values

- Difficult to model, · Tunable

therefore empirical dampers

required

- Subject to dielectric

breakdown, limits

energy density $(0.001$

$\mathrm{J} / \mathrm{cm}^{3}$ )

- Particulate settling

field

- Friction

devices

- Shock

absorbers

- Limited lifetime due

to fluid thickening

- Low stress

- Low response speed

- Electric

- Artificial

field

muscles

various technologies - Low efficiency

- High strains · Low durability

- Micro/nano

positioners 
transformation temperature ${ }^{145}$. Ease of fabrication allows for use of SMAs in a variety of form factors, such as thin films, wires, and particles ${ }^{150}$. Other benefits include high recovery forces, large recoverable output strains, different actuation modes (bending, torsion, etc.), and high work output per unit mass ${ }^{146}$; however, slow response times and low efficiency limit practical applications of SMAs ${ }^{146}$.

Magnetostriction. Similar to the piezoelectric effect, magnetostriction is deformation of a body in response to a change in magnetization, brought about by application of a magnetic field ${ }^{157}$. Interestingly, all materials that are magnetic exhibit some degree of magnetostriction; however, giant magnetostriction occurs in a much smaller population of those containing rare earth elements ${ }^{157}$. Upon application of an external magnetic field, magnetic domains within the material that are aligned with the external field will grow, while other domains will be reduced ${ }^{155}$. One advantage of magnetostrictive materials is low process temperatures during fabrication ${ }^{158}$. Magnetostrictive materials are found in a variety of applications, including vibration control, sonar transducers, and linear motors ${ }^{155}$.

Electrorheological (ER) or magnetorheological (MR) fluids. ER and MR fluids are terms given to mixture of small $(\sim 1-10 \mu \mathrm{m})$ particles that are dispersed at $\mathrm{wt} \%$ of up to $50 \%$ within an insulating base oil (typically mineral or silicone oil) ${ }^{144,145}$. When an electric or magnetic field is applied transverse to the direction of fluid motion, an interaction is caused between the particles, oil, and applied field resulting in increase in resistance to fluid flow ${ }^{144}$. In the absence of an electric or magnetic field, the fluid/particle suspensions exhibit Newtonian fluid characteristics with shear stress $(\tau)$ given by $\tau=\eta \dot{\gamma}^{145}$. Application of an electric field $(E)$ to an ER fluid, or a magnetic 
field $(H)$ to a MR fluid, results in formation of particle "chains" or fibrils in the direction of the field ${ }^{145}$. When fluid flow normal to the direction of the electric or magnetic field is applied, fibril chains are broken and must reform. Continuous breaking and reforming of chains causes a force that resists the fluid motion, resulting in a controllable shear stress ${ }^{145}$. The majority of industrial applications of ER and MR fluids involve exploiting the controllable shear stress of these fluids to vary the coupling or load transfer between

moving parts ${ }^{145}$; however, ER and MR fluids must keep particles in suspension within the fluid, requiring the use of additional chemicals or mechanical agitation.

\subsection{OPTICALLY DRIVEN ACTUATORS}

Because photons cannot induce large forces in any solid state actuation materials, optically driven actuators are a relatively new addition to the library of micro and nanoactuation technologies. Nonetheless, inherent advantages of optically driven materials have proven useful on micro and nanosize scales, and thus are considered critical components in a variety of future nanoscale-sized devices ${ }^{159}$. As compared to electrically/thermally/acoustically/chemically driven actuators, optical actuation has advantages including wireless/remote actuation, displacement control using phase/intensity/polarization effects, electrical-mechanical decoupling allowing for separation of actuator and power source (ideal for use in hazardous or explosive environments), good scaling capabilities, and massive parallel actuation of device arrays from a single light source. Furthermore, optically driven materials can be classified into two categories based on their type of response, these categories are direct or indirect ${ }^{160}$. Direct methods use active photosensitive materials in which photon absorption causes an internal molecular change resulting in alterations of material properties, an example being 
use of light to alter electrostatic forces and cause actuation ${ }^{160}$. Advantages of direct actuation include faster response, simpler device design, and lower light power requirements than indirect methods to induce actuation responses ${ }^{161}$. A major drawback, however, is that along with the lower power requirements, direct actuation mechanisms entail corresponding low generated forces. Since indirect photo-mechanical actuation utilizes secondary effects (photon-induced heating, photon-generated electrical current, or resistivity change ${ }^{160,161}$ ), indirect methods typically generate more actuation force than direct mechanisms ${ }^{160}$. For example, IR illumination-induced heating of an encapsulated gas, or causing a phase change in a material is an example of indirect optical actuation ${ }^{160}$. The heating and/or phase transformations mechanisms that indirect methods utilize, however, typically result in slower responses than direct actuation ${ }^{160}$. As device size shrinks, however, quasi-fundamental scaling dependencies result in improved characteristics of indirect photo-mechanical actuation, making these methods as well as direct methods appealing for use in a variety of devices ${ }^{162}$.

In spite of advantages presented by optical actuation and novel device possibilities, only a select number of systems exhibit these types of direct and indirect effects. The most well-known systems include: (1) azobenzenes, (2) chalcogenide glasses, (3) liquid-crystal elastomers, (4) photo-thermal actuation, (5) charge-induced photo-mechanical effect, (6) radiation pressure, and (7) optically-triggered shape memory. Table 2.2 presents a summary of major advantages and disadvantages in these materials, followed by several material-specific discussions. 


\section{Table 2.2. Comparison of general characteristics between different photo-actuation}

mechanisms.

\begin{tabular}{|c|c|c|}
\hline Systems & Advantages & Disadvantages \\
\hline Azobenzene $^{159}$ & $\begin{array}{l}\text { Robust } \\
\text { Chemically stable }\end{array}$ & $\begin{array}{l}\cdot \text { Low efficiency } \\
\left(\sim 10^{-16} \% \text { experimental }\right) \\
\cdot \text { Long relaxation time (hours) }\end{array}$ \\
\hline $\begin{array}{l}\text { Chalcogenide } \\
\text { glasses }^{161,163,164}\end{array}$ & $\begin{array}{l}\cdot \text { Highly reversible } \\
\cdot \text { Effect is wavelength selectable } \\
\cdot \text { Multitude of photo-induced effects } \\
\text { possible }\end{array}$ & $\begin{array}{l}\cdot \text { Slow response times } \\
\cdot \text { Small strains }\end{array}$ \\
\hline $\begin{array}{l}\text { Liquid-crystal } \\
\text { elastomers } 165\end{array}$ & $\begin{array}{l}\text { Fast response times }(\mathrm{ms}) \\
\text { - Large deformation }\left(>60^{\circ} \text { bend angles) }\right.\end{array}$ & $\begin{array}{l}\text { - Aging limits lifetime } \\
\text { - Many require liquid environments }\end{array}$ \\
\hline Photo-thermal ${ }^{166,167}$ & $\begin{array}{l}\text { - Larger forces possible than other } \\
\text { optical methods } \\
\text { - Ability to induce phase changes in } \\
\text { material for increased actuation } \\
\text { - Good size scaling characteristics }\end{array}$ & $\begin{array}{l}\cdot \text { Slow } \\
\cdot \text { Inefficient } \\
\cdot \text { Requires cooling for reverse } \\
\text { transformation } \\
\cdot \text { High illumination intensities }\end{array}$ \\
\hline $\begin{array}{l}\text { Charge-induced } \\
\text { photo-mechanical } \\
\text { effect }^{167}\end{array}$ & $\begin{array}{l}\text { - Faster response than photo-thermal } \\
\text { - High frequency }\end{array}$ & - Limited to nanoscale devices \\
\hline $\begin{array}{l}\text { Radiation } \\
\text { pressure }^{160,168}\end{array}$ & $\begin{array}{l}\text { - Ability to increase force via reflective } \\
\text { coatings }\end{array}$ & $\begin{array}{l}\text { Limited to nano-scale devices } \\
\text { Potential opto-thermal stability } \\
\text { problems }\end{array}$ \\
\hline $\begin{array}{l}\text { Optical triggered } \\
\text { shape memory }{ }^{169}\end{array}$ & $\begin{array}{l}\text { Exhibit long term stability } \\
\text { Strain-recovery rate is comparable to } \\
\text { that of photo-responsive shape- } \\
\text { memory materials }\end{array}$ & $\begin{array}{l}\text { - Requires long illumination times } \\
(\sim 1.5 \text { h) to active shape recovery }\end{array}$ \\
\hline
\end{tabular}

Radiation pressure. While it has long been considered for use in spacecraft propulsion $^{170,171}$, the advent of device scaling (using MEMS/NEMS processing) made radiation pressure a possible actuation technology terrestrially. Compared to other forces, effects of radiation pressure are almost insignificant viewed on macroscale scales, thus making space-based applications ideal. For example, radiation-induced effects measured on the Pioneer space probes launched in the early 1970's showed a sun-induced acceleration substantially less than $8 \times 10^{-8} \mathrm{~cm} / \mathrm{s}^{2}{ }^{172}$. Additionally, radiation pressure effects must be taken into account with orbiting satellites - such perturbations have been shown to introduce errors into global positioning system (GPS) readings ${ }^{173}$. 
While the forces generated are incredibly small, electro-magnetic radiation nonetheless exerts a pressure on an exposed surface. The principle behind this phenomena is that light contains momentum, and when a photon is either reflected or absorbed by an incident body, momentum is transferred in the form of radiation pressure $^{168}$. Momentum contained within a single photon $\left(p_{p h}\right)$ is given in equation (2.1), where $h$ is Planck's constant, $c$ is speed of light, and $v$ is optical frequency ${ }^{160}$. Based on conservation of momentum, therefore, a reflective body will experience a force $\left(F_{r e f}\right)$ equal to two times the illumination power $(P)$ divided by the speed of light [equation $(2.2)]^{160}$. Interestingly, in radiation absorption vice reflection- a surface will not experience any photon recoil momentum, and thus realize a force half that of the reflective surface. These equations show that the magnitude of the force is directly proportional to the illumination power.

$$
\begin{aligned}
& p_{p h}=h v / c \\
& F_{\text {ref }}=\left(d p_{\text {total } p h} / d t\right)=2 P / c
\end{aligned}
$$

On MEMS-/NEMS-sized scales, small radiation pressures can have significant impacts. For example, Sulfridge et al demonstrate a bistable MEMS device ${ }^{168}$. Building on earlier radiation-based cantilever actuation devices ${ }^{174,175}$, they demonstrated a $1000 \mu \mathrm{m}$ long by $1 \mu \mathrm{m}$ wide bistable beam with a buckling displacement of $\sim 3 \mu \mathrm{m}$ and a force required to toggle the device estimated to be $\sim 50 \mathrm{pN}^{168}$. Based on equation (2.2), the $10 \mathrm{~mW}$ laser source used would provide $\sim 67 \mathrm{pN}$, enough to toggle the device. 
Photo-thermal. The most prevalent and mature optically-induced actuation mechanism is photo-thermal (literature on non-photo-thermal methods did not start appearing until the $1990{ }^{\prime 168}$ ). Photo-thermal actuation is simply the transduction of electro-magnetic energy into heat, thus resulting in mechanical movement. While thermal responses can potentially be slower than those of other optical actuation methods, the combination of potentially large forces and simple device design makes photo-thermal an attractive actuation mechanism. Additionally, ease of size scaling results in means to design high-speed micro and nanosized photo-thermal actuators such that responses are either on par with or exceed other optical other mechanisms. Finally, since no material is perfectly reflective ${ }^{176}$, some electro-magnetic energy absorption will occur in any optically-powered device inevitably resulting in some, albeit small amount, of photothermal actuation.

Photo-thermal based actuators can take a number of different geometries; the most common methods include some combination of bimorph or differential thermal expansion setup ${ }^{177,178}$, encapsulated material that undergoes a liquid to gas phase change ${ }^{179,180}$, or simple thermal expansion/contraction in an unrestricted body ${ }^{53-55}$. For example, in a paper on bent-beam thermal actuators, Que et al by using localized thermal stresses were able to demonstrate forces up to $\sim 1 \mathrm{mN}$, two to three orders of magnitude greater than other comparable devices ${ }^{181}$. While they utilized electro-thermal heating, a photo-thermal process could be easily substituted, providing both greater control and potentially increased force generation. In phase change applications, localized heating of an encapsulated liquid has been shown to induce a phase change resulting in lifting of a polysilicon plate $\sim 140 \mu \mathrm{m}^{182}$. 
Chalcogenide glasses. Chalcogenide glasses are based on the addition of elements such as $\mathrm{Ge}, \mathrm{As}, \mathrm{Sb}, \mathrm{Ga}$ to chalcogen elements ( $\mathrm{S}, \mathrm{Se}$, and $\mathrm{Te}$ ), which can be processed into a variety of forms, such as fibers, thin films, and bulk samples ${ }^{183}$. These materials demonstrate intrinsic photosensitivity leading to optically-induced changes ${ }^{184}$. Generally considered low-phonon energy materials that are transparent (visible to infrared range), chalcogenide glasses can be further doped with the addition of rare earth elements, leading to numerous different types of photo-induced effects ${ }^{183}$.

Among the dozens of prominent photo-induced effects observed so far in chalcogenide glasses ${ }^{164}$, a variety are ideal for exploitation in actuation devices. The most relevant include: (1) Giant photo-expansion, whereas volume expansions of $\sim 5 \%$ in a $50 \mu \mathrm{m}$ thick $\mathrm{As}_{2} \mathrm{~S}_{3}$ film under $10^{3} \mathrm{~W} / \mathrm{cm}^{2}$ illumination have been reported ${ }^{163}$. While still somewhat speculative, the giant photo-expansion is attributed to volume expansion and fluidity ${ }^{185}$. (2) Contrasting to photo-induced expansion, photo-induced contraction has also been demonstrated. Using Ge-based glasses, they were able to show photo-induced volume contraction and photo-bleaching as opposed to the expansion and photodarkening inherent to As-based glasses ${ }^{186}$. (3) An anisotropic opto-mechanical effect has also been demonstrated, which is due to the preferential absorption and/or reflection of polarized light in an isotropic chalcogenide sample ${ }^{161}$. For example, Stuchlik et al fabricated a clamped microcantilever with an amorphous chalcogenide layer ${ }^{184}$. They found that exposure to and absorption of linearly-polarized light subsequently induced a differential anisotropic change in cantilever strain resulting in mechanical displacement. Interestingly, light polarized to parallel to the cantilever caused it to bend towards the light, while polarized light perpendicular to the cantilever caused a repulsive (downward) 
displacement. On a $381 \mu \mathrm{m}$ long cantilever with a $1 \mu \mathrm{m}$ thick $\mathrm{As}_{40} \mathrm{~S}_{35} \mathrm{Se}_{25}$ film, maximum

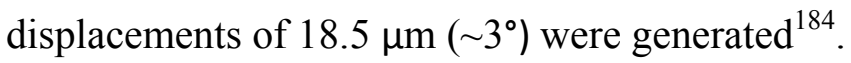

Liquid crystal elastomers. These are materials that utilize rubbery elastic properties of polymer elastomers and self-organizational properties of liquid crystals ${ }^{187}$. This combination gives liquid crystal elastomers (LCEs) the ability to alter their shape by as much as $400 \%$ in response to environmental changes, such as chemical, temperature, and light ${ }^{160}$. LCEs are finding applications in various biomedical devices, lenses, and artificial muscle actuators ${ }^{188}$. Based on large dimensional changes that can result from electrochemical doping of assorted polymers (such as polypyrroles, polyanilines, polyalkylthiophenes, and polyarylvinylenes), conducting polymers can be made to exhibit actuation effects ${ }^{44}$. Dimensional changes in conducting polymers are attributed to the exchange of ions from the conducting polymer to the surrounding media, which is driven by an applied voltage ${ }^{144}$. Conducting polymers are being investigated for use in various sensors and micro/nano manipulation applications ${ }^{189}$. Assorted conducting polymers show actuation performance on par with stroke, efficiency, and force on par with human muscles ${ }^{160}$. Drawbacks include suffering from Faradiac processes including ionic diffusion, creating limitations on actuation rate and cycle life ${ }^{160}$. Many conducting polymer actuators also require a liquid environment, presenting difficulties in dry or vacuum environments ${ }^{160}$.

While all of these optically-driven material systems are both incredibly interesting and of high scientific importance, this dissertation introduces a new type of photomechanically actuated system that is based on carbon nanostructure/elastomer composites. Actuation is witnessed only through the homogeneous dispersion of various 
types of carbon nanostructures within a PDMS silicone elastomer matrix. Upon IR illumination, photons are absorbed by the carbon nanostructures and heat energy is transferred to the polymeric chains, resulting in reversible and repeatable opticallyinduced elastic expansion and contraction. The following chapter describes a GNP/PDMS system with an extraordinary optical-to-mechanical energy conversion factor of 7 $-9 \mathrm{MPa} / \mathrm{W}$. This conversion factor is not only far greater than any form of carbon/PDMS samples tested to date, but also significantly larger than most materials that undergo photo-mechanical effects. 


\section{CHAPTER 3 \\ GRAPHENE-NANOPLATELET-BASED PHOTO-MECHANICAL ACTUATORS}

\subsection{INTRODUCTION}

This chapter introduces a simple polymer composite system whose photo-mechanical responses to near infrared light are realized solely by incorporation of homogeneous dispersion of GNPs within a PDMS elastomer matrix. Actuation responses of GNP/PDMS composites depend on initial applied pre-strain: at low levels (3-9\%), samples showed reversible expansion; at moderate levels $(\sim 10 \%)$, samples yielded negligible effect; and at high levels (15-40\%), samples exhibited reversible contraction. For GNP/PDMS composites, these correspond to a photo-mechanical stress change 2.4 to 3.6 times greater than any other carbon form tested. These stress changes reported are usable and recoverable work done by the actuators. An optical-to-mechanical energy conversion factor $\left(\eta_{\mathrm{M}}\right)$ of 7-9 MPa/W obtained during testing showed an extraordinary photo-mechanical effect larger than not only all other carbon-based composites, but also any other material that exhibits photo-mechanical effect. These intriguing results will help to inspire continued research towards the expansion of GNP-based micro and nanooptomechanical systems. 


\subsection{EXPERIMENTAL SETUP}

Figure 3.1(a) shows a scanning electron microscope (SEM) image of GNPs deposited on a silicon wafer. During close examination, the GNPs were verified $\leq 3$ layers, with diameters in the range of 1-2 $\mu \mathrm{m}$. Figure 3.1(b) shows Raman spectroscopy comparison of plain PDMS polymer, GNP/PDMS composites, and CNT/PDMS composites. The Raman peaks of the PDMS are clearly visible in all three curves of Figure 3.1(b); the $1582 \mathrm{~cm}^{-1}$ and $2700 \mathrm{~cm}^{-1}$ peaks correspond to graphene. The radial breathing mode (RBM), graphite $(G)$, and CNT-2D band $\left(G^{\prime}\right)$ are also clearly seen. The SEM and Raman spectroscopy confirmed high purity of these systems. Figure 3.1(c) presents the experimental setup schematic that shows test sample vertical orientation of

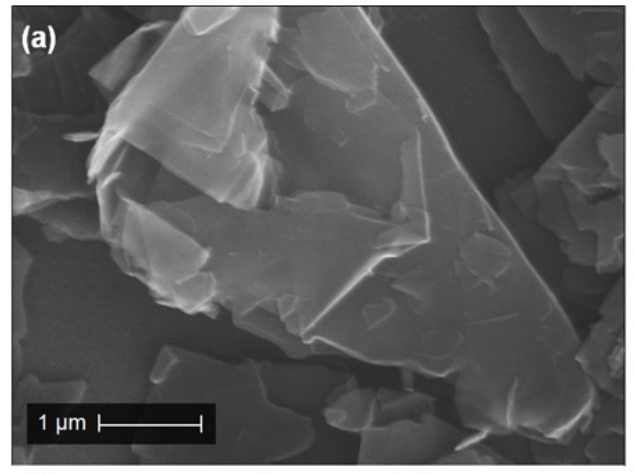

(c)
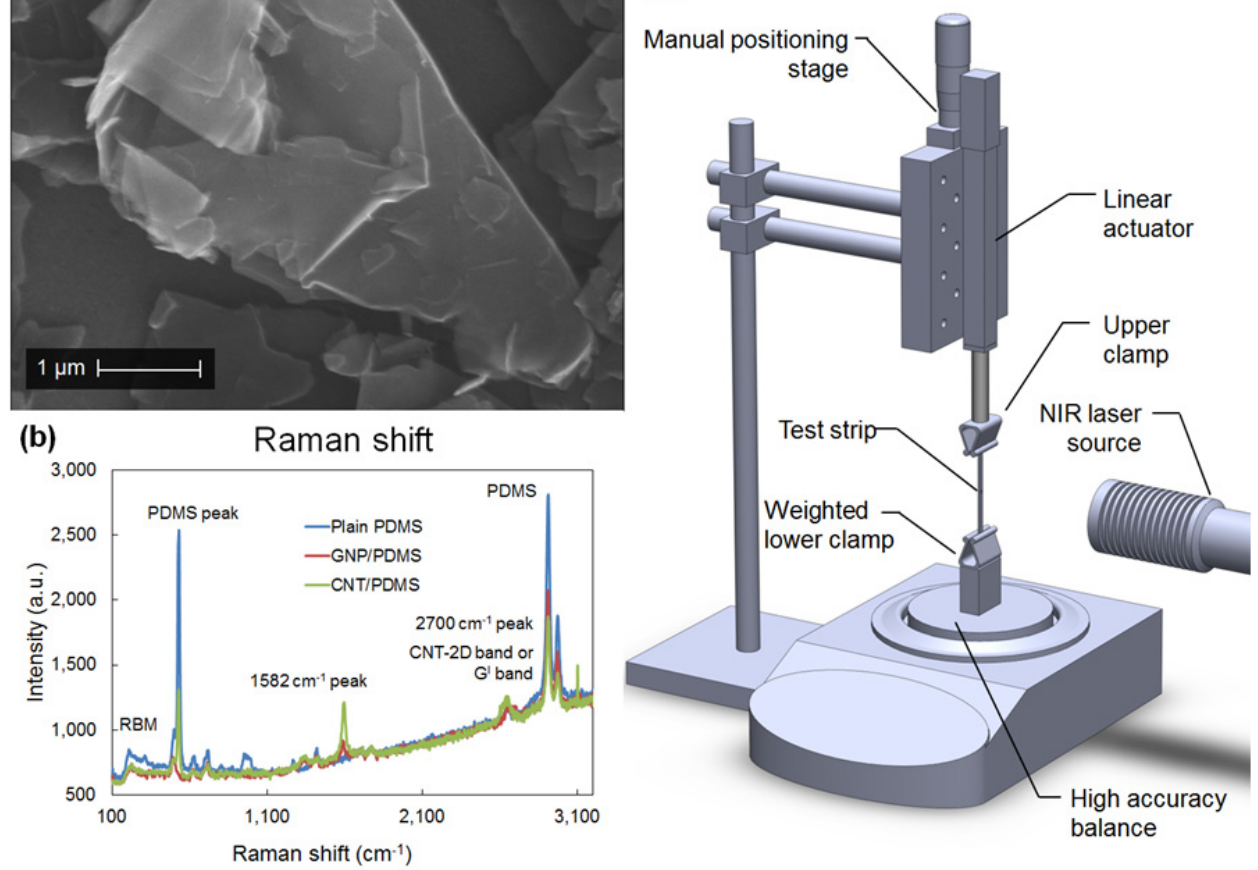

Figure 3.1. GNP/PDMS photo-mechanical actuator characterization and testing. (a) SEM image of GNPs. (b) Raman spectroscopy shift of PDMS, GNP/PDMS and CNT/PDMS. (c) Layout of test dynamometer. 
the test nanocomposite sample within the testing dynamometer assembly. See Appendix A for further information regarding composite sample preparation and test system setup.

\subsection{PHOTO-MECHANICAL STRESS AND KINETICS}

Figure 3.2(a) shows the typical photo-mechanical actuation and relaxation kinetics during a 3\% pre-strain test. For each pre-strain (3-40\%), test composites underwent 5 cycles of NIR illumination on for $60 \mathrm{~s}$, followed by NIR illumination off for $30 \mathrm{~s}$. The magnitude of actuation was highly repeatable for each test sample. Photomechanical responses started at time $(t)=0 \mathrm{~s}$ and became saturated at $t=5 \mathrm{~s}$. Similarly, once the light is switched off, actuators undergo relaxation in $t=5 \mathrm{~s}$. This is significantly fast actuation compared to past reports on CNT/PDMS actuators where the actuation achieved saturation at $t=10-15 \mathrm{~s}^{62}$. The dependency of actuation and relaxation responses as a function of time were also examined, with experimental data fit to simple exponential functions by incorporation of a fitting parameter $(\tau)$. The experimental actuation data was fitted with a simple exponential function of $1-\exp [-(t / \tau)]$, while the relaxation data was fitted with $\exp [-(t / \tau)]$. Both actuation and relaxation responses followed Debye characteristics ${ }^{62}$. Figure 3.2(b) shows the experimental actuation response and associated fitting function when $\tau \approx 1.7 \mathrm{~s}$. Figure 3.2(c) shows the experimental relaxation response and fitting function when $\tau \approx 2 \mathrm{~s}$. When normalized to account for the magnitude of change in stress, actuation and relaxation responses for all GNP/PDMS test composites were nearly identical. The fast actuation and relaxation of GNP/PDMS composites in both expansive and contractive modes suggests unique 

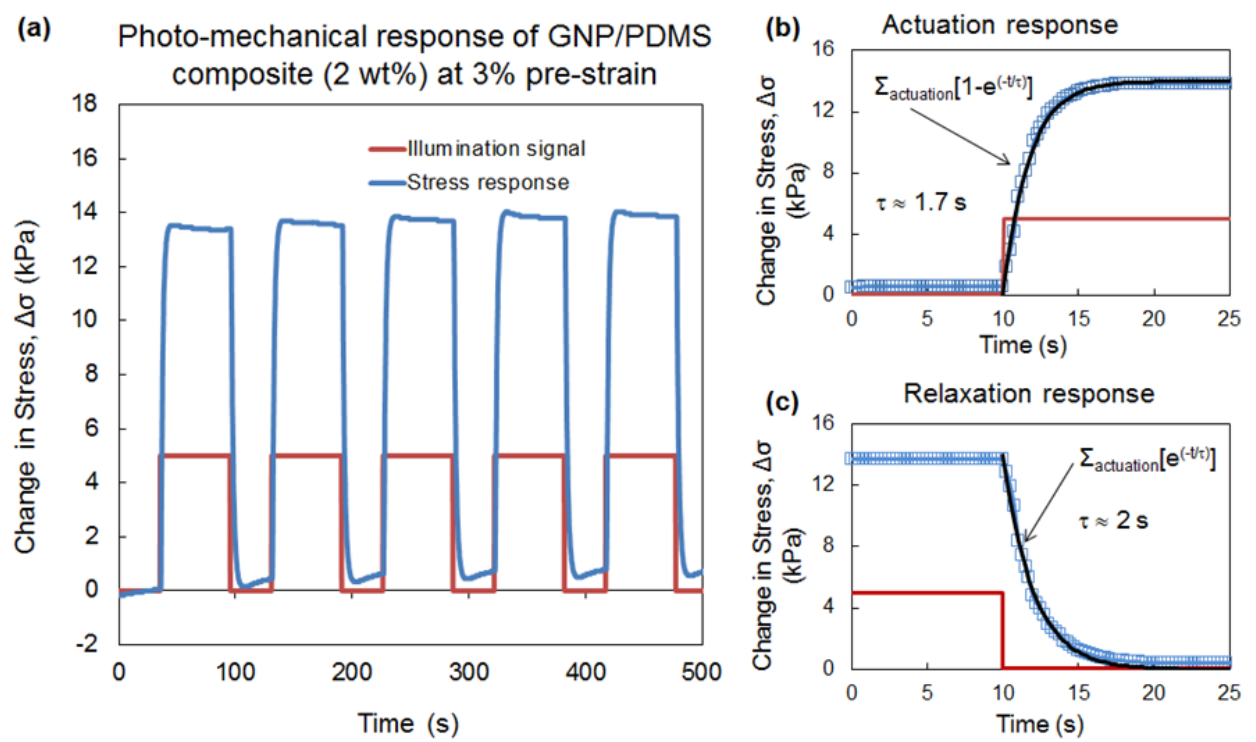

Figure 3.2. Photo-mechanical responses and actuation kinetics of GNP/PDMS composites. (a) Typical photo-mechanical stress response of GNP/PDMS actuator 3\% pre-strains. (b-c) Actuation and relaxation of GNP/PDMS actuator, respectively.

actuation mechanism for GNP/PDMS actuators. Unlike CNT/PDMS actuators which follow a compressed exponential function for actuation ${ }^{62}$, GNP/PDMS actuators were found to not only follow a simple exponential function during both actuation and relaxation, but also exhibit about a three times faster response. Secondly, the actuation kinetics of the CNT/PDMS actuators followed a compressed exponential function only above the percolation threshold ${ }^{62}$. For values lower than the percolation threshold, at $\sim 0.02 \mathrm{wt} \%$ concentration of the nanotubes in the PDMS matrix, the actuation was far slower with time constants of actuation greater than $20 \mathrm{~s}$ for achieving saturation ${ }^{62}$. This shows the dependence of actuation kinetics on the number of interconnected nanotubes in the matrix. In marked contrast and regardless of the sample concentrations, GNP/PDMS actuators exhibit fast actuation and relaxation and the quality of the fit of the 
experimental data follows a simple actuation and relaxation. This suggests that the energy transfer associated with photon absorption leading to subsequent expansive or contractive actuation and relaxation of the polymeric chains is a fast event which is unique to graphene and therefore a unique photo-mechanical mechanism. Such fast actuation and relaxation cannot be explained by simple thermodynamic considerations of steady-state heat flux from irradiated GNPs causing saturation of the photo-mechanical response. Identical response times for actuation and relaxation also suggest constant viscosity and modulus therefore maintaining the original configuration of the polymer. Future fast pump-probe experiments or AFM experiments with functionalized graphene sheets with single chain polymers can throw some light on the extraordinary energy transfer needed to explain this fast actuation and relaxation.

Starting with a plain PDMS elastomer [Figure 3.3(a)] and progressing from 0.1 to $5 \mathrm{wt} \%$ concentration GNP/PDMS composites [Figure 3.3(f)], each plot shows the photomechanical response to $808 \mathrm{~nm}$ NIR illumination for a single 60 -second cycle. Since the optical loss of PDMS in the NIR region is $<0.5 \mathrm{~dB} / \mathrm{cm}^{190}$, the negligible response in the plain PDMS sample [Figure 3.3(a)] was expected. However, by a concentration of $0.1 \mathrm{wt} \%$ GNPs [Figure 3.3(b)], the photo-mechanical effect becomes clearly observable through telltale expansion and contraction of the actuator. As all the composites exhibited, low pre-strain values resulted in film expansion and thus positive-induced stress, while high pre-strain values resulted in compression and thus negative change in stress. More concisely stated, weakly stretched composites show reversible expansion while highly stretched composites show reversible contraction ${ }^{51}$. From GNP 
Photo-mechanically induced stress response of various GNP/PDMS concentrations
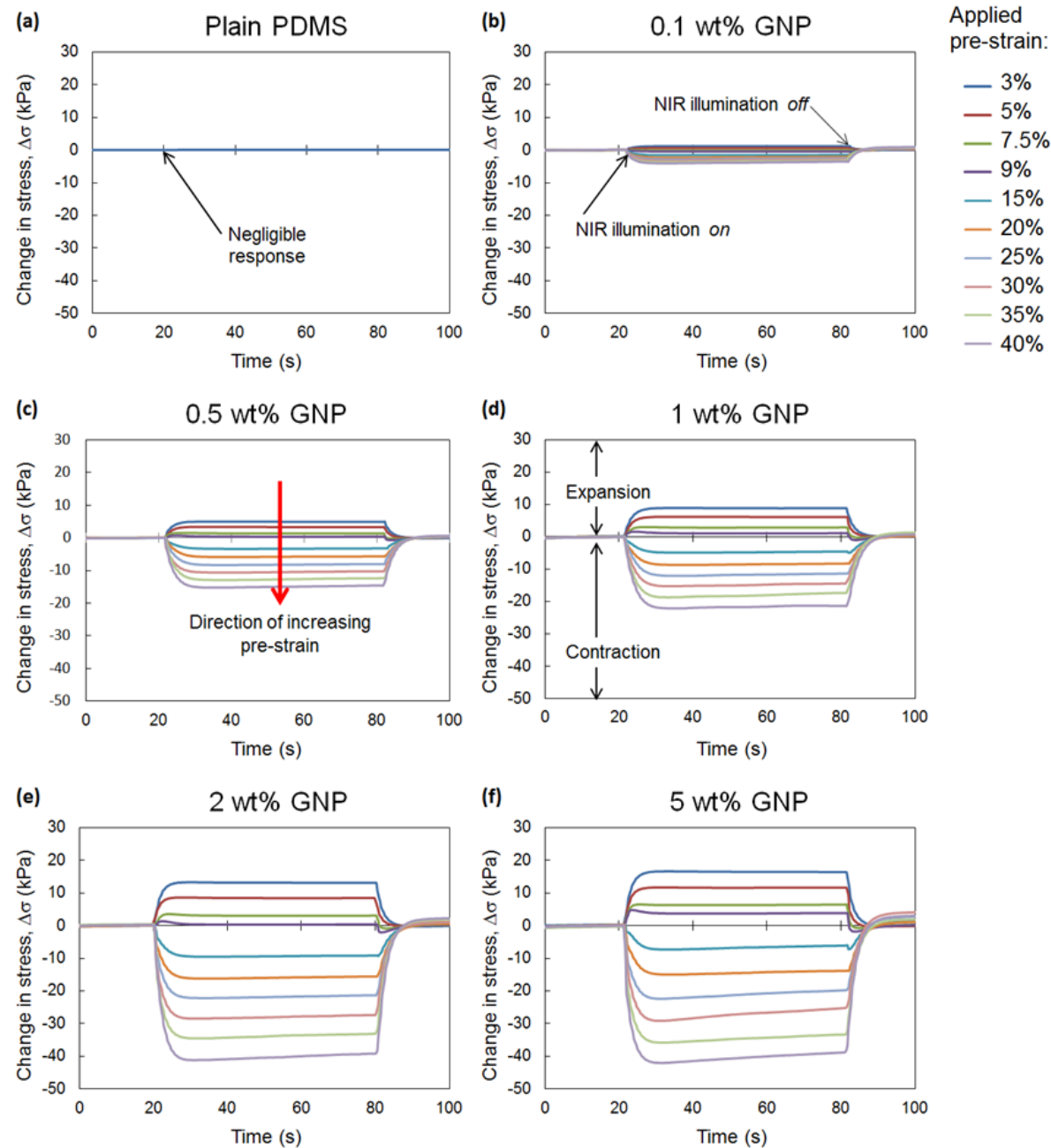

Figure 3.3. Comparison of increasing photo-mechanically induced stress change in GNP/PDMS composites as a result of NIR illumination for increasing GNP concentrations. (a) Plain PDMS. (b) $0.1 \mathrm{wt} \%$ GNP. (c) $0.5 \mathrm{wt} \%$ GNP. (d) $1 \mathrm{wt} \%$ GNP. (e) $2 \mathrm{wt} \%$ GNP. (f) $5 \mathrm{wt} \%$ GNP.

concentrations of $0.1 \mathrm{wt} \%$ to $2 \mathrm{wt} \%$, [Figures $3.3(\mathrm{~b})-(\mathrm{e})$ ], the magnitude of photoinduced stress was observed to increase, reaching almost four orders of magnitude greater than the pristine PDMS polymer. By a concentration of $2 \mathrm{wt} \%$, however, the photomechanical response of the GNP/PDMS composites had become saturated. Comparison 
with higher GNP concentration of $5 \mathrm{wt} \%$ [Figure 3.3(f)] showed only a marginal increase in magnitude of the stress. Therefore, photo-mechanical response of the $2 \mathrm{wt} \%$ GNP/PDMS composite sample was used as the benchmark for comparison throughout this chapter. The change from expansion to contraction in all samples is likely a result of orientational effects, similar to those seen in CNT-based actuators ${ }^{51}$. As applied prestrains increase overall test sample length, the GNPs within the PDMS polymer become more aligned/re-arranged with respect to one another. As a result, the macroscopic magnitude of photo-mechanical response is amplified.

To compare the photo-mechanical responses of GNP/PDMS composites with other forms of carbon/PDMS composites, test samples were fabricated for GO/PDMS, CNT/PDMS, and pyrolytic carbon black (CB)/PDMS using identical fabrication methods. All samples underwent identical testing procedures to the GNP/PDMS composites, Figures 3.4(a)-(d) present these results. All forms of carbon displayed similar photo-mechanically induced expansion/contraction stress responses as the GNPs, although with varying magnitudes. Of these samples, GO/PDMS [Figure 3.4(a)] showed the smallest amount of actuation, from $+2 \mathrm{kPa}$ ( $3 \%$ pre-strain) to $-9 \mathrm{kPa}(40 \%$ pre-strain). This result also shows that studies of graphene-composites derived from GO materials are quite different from single, bi-layer, or few layer graphene. The magnitude of induced stress change in CNT/PDMS [Figure 3.4(b)] was slightly higher, from $+4 \mathrm{kPa}$ (3\% prestrain) to $-10 \mathrm{kPa}$ (40\% pre-strain). Finally, CB/PDMS [Figure 3.4(c)] exhibited a stress change from $+7 \mathrm{kPa}$ ( $3 \%$ pre-strain) to $-14 \mathrm{kPa}(40 \%$ pre-strain). The GNP/PDMS response [Figure 3.4(d)] magnitude of stress-induced change ranged from $+14 \mathrm{kPa}$ to -36 $\mathrm{kPa}$, an average of 4.5 times larger than that of GO/PDMS, 3.6 times CNT/PDMS, and 
Photo-mechanically induced stress response of various

PDMS composites (2 wt\%)
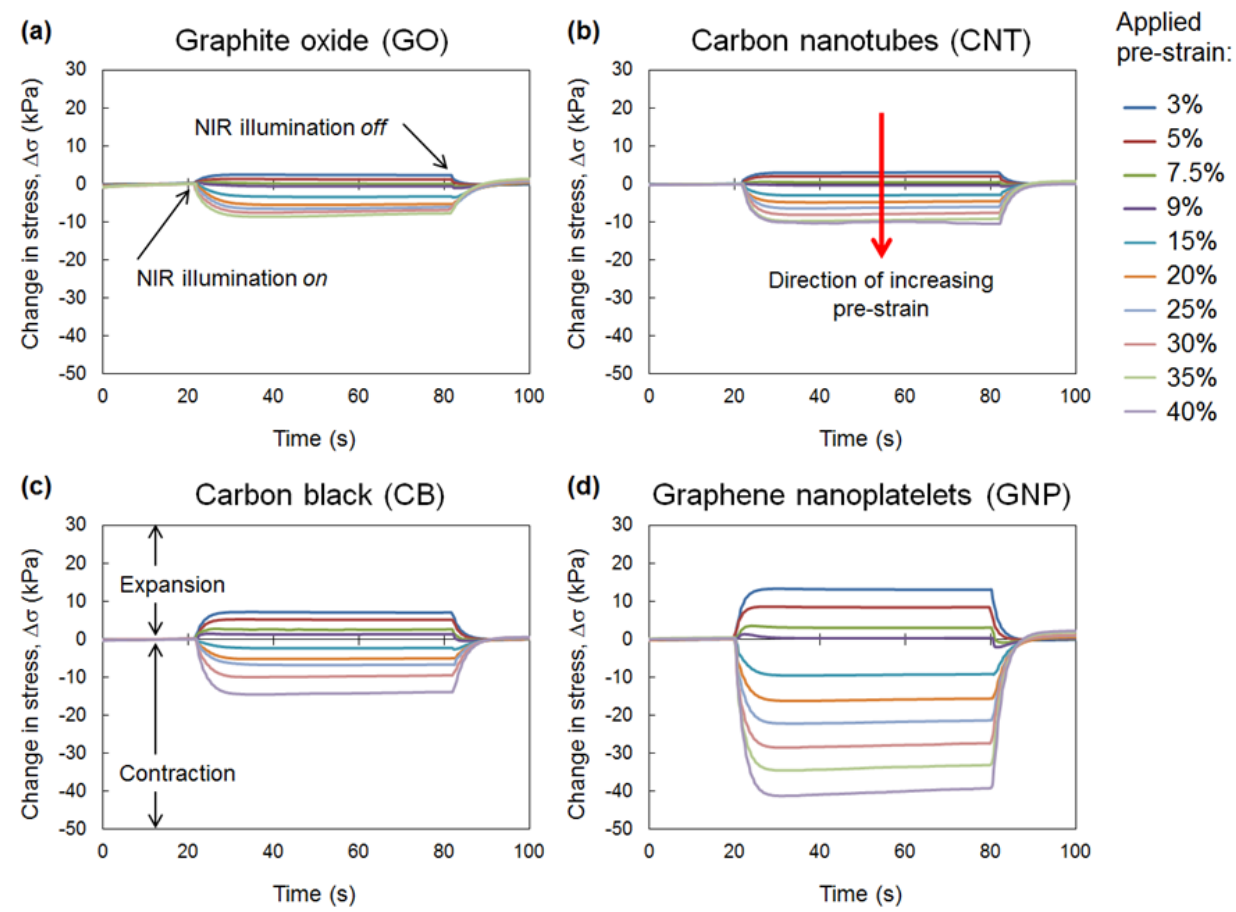

Figure 3.4. Comparison of photo-mechanically induced stress change in $2 \mathrm{wt} \%$

composites of various carbon forms. (a) Graphite oxide. (b) Carbon nanotubes. (c)

Carbon black. (d) Graphene nanoplatelets.

2.4 times CB/PDMS for the same fabrication methods. The similar magnitude of stress change seen in CNT/PDMS and CB/PDMS demonstrate the lack of dispersion of CNTs in the PDMS matrix acting like particles similar to $\mathrm{CB}$ rather than tubular filaments is due to short mixing times. Past reports have shown much larger stress increases for MWNTs than seen here ${ }^{62}$. In those studies, the MWNTs were shear mixed in a high shear laboratory mixer for minimum of 24 hours in the PDMS composite before fabricating the actuator. The resulting stress of $\sigma_{\max } \approx 50 \mathrm{kPa}$ at $8 \mathrm{~mW}^{51,62}$ of absorbed power $\left(P_{\text {effective }}\right)$ giving rise to a calculated $\eta_{\mathrm{M}}$ for CNTs of $6.25 \mathrm{MPa} / \mathrm{W}$. In the present study, the 
graphene was only shear mixed for 5 min before fabricating the actuator to obtain this extraordinary $\eta_{\mathrm{M}}$ of $7-9 \mathrm{MPa} / \mathrm{W}$. It may be possible to obtain much larger $\eta_{\mathrm{M}}$ values with longer mixing times. In the present study however, this same short-time shear mixing procedure was followed for all carbon/PDMS composites, to enable a standardized comparison between different types of carbon/PDMS actuators. This procedure shows the relative ease at which the plate-like GNPs disperse in an elastomeric matrix such as PDMS compared to other carbon forms. The plate-like structure of GNPs may enable the individual layers to slide against the polymer, thus achieving rapid and uniform dispersion compared to filament-like CNTs that tend to roll up into particles in the short mixing time. For the GNP/PDMS actuators, when the actuation is translated into a stroke, this corresponds to a total strain of $\sim 2-5 \%$, significantly larger than lattice expansion or contraction alone ${ }^{191}$. It is clear that the photo-mechanical actuation of graphene is quite impressive compared to its other carbon counterparts.

Figure 3.5(a) shows a comparison of the photo-mechanical response for three $2 \mathrm{wt} \%$ GNP/PDMS samples at pre-strains of $3 \%$ and $40 \%$. As shown in the figure, actuation and relaxation are relatively consistent throughout the samples with marginal dependence on the pre-strains applied, both in magnitude of actuation as well as response time. Figure 3.5(b) shows the photo-mechanical stress response versus applied pre-strain, with $\pm 1 \mathrm{kPa}$ standard deviation error bars. At low pre-strains (under 15\%), the standard deviation is less than $1.5 \mathrm{kPa}$, as pre-strain increases, so does the standard deviation, reaching a maximum of $\pm 5.4 \mathrm{kPa}$ at $40 \%$ pre-strain. This suggests that as pre-strains increase, the orientational ordering of GNPs results in larger contraction of the actuator or better dispersed/aligned composites. Figure 3.5(c) presents the change in Young's 
modulus $(E)$ as a function of GNP fractions dispersed in the polymer. A Young's modulus increase of almost twice that of pristine PDMS elastomer was obtained ${ }^{192}$, and thus higher wt $\%$ GNP actuators are also stiffer. These results suggest that GNPs in PDMS produced not only a stiffer actuator but also an extraordinary optical-tomechanical energy conversion of 7-9 MPa/W. It is common knowledge that any type of energy conversion is associated with heating effects with only a fraction of energy used to provide useful work. Due to this effect, the macroscopic steady-state temperature increase in the actuators was studied.
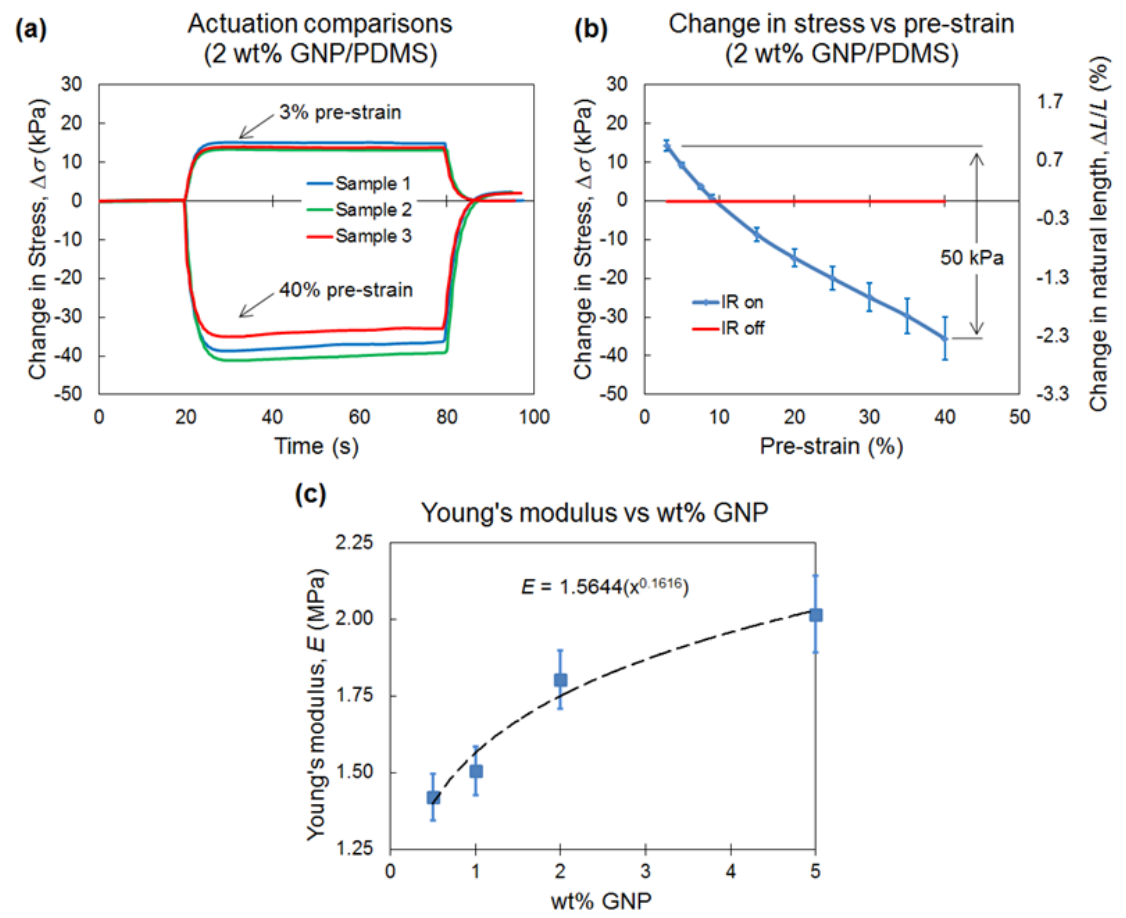

Figure 3.5. GNP/PDMS actuator characteristics. (a) Comparison of actuation response from 3 samples at 3\% and $40 \%$ pre-strains. (b) Average actuation response versus prestrain with \pm 1 standard deviation error bars. (c) Increase in Young's modulus of the actuator with increase in GNP fraction. 


\subsection{TEMPERATURE RELATIONSHIP}

Laser irradiation on the GNP/PDMS composites was found to increase actuator temperature at the irradiation spot. The temperature decrease as a function of distance from the illumination source, therefore, was characterized for various GNP/PDMS composite concentrations. Figure 3.6 details this temperature rise for $0 \%$ pre-strain (sample length $50 \mathrm{~mm}$ ) as well as $40 \%$ pre-strain (sample length $70 \mathrm{~mm}$ ). As expected, the highest temperature increase also occurs at the highest GNP concentration (5\% GNP, $0 \%$ pre-strain). $\mathrm{A} \sim 75^{\circ} \mathrm{C}$ temperature rise quickly diminishes as distance from the illumination point increases. As pre-strain and thus sample length increase, the amount of graphene exposed to the fixed size irradiation spot decreases due to orientational ordering; and, therefore, thermal effects also start to diminish. At $40 \%$ pre-strain, the maximum temperature increase has fallen to $\sim 60^{\circ} \mathrm{C}$, a $15^{\circ} \mathrm{C}$ change compared to the unstrained sample. It should be pointed out that at large pre-strain, the overall magnitude of the actuation increase, while the temperature rise in the actuator decrease. This outcome possibly indicates that thermal effects may not be the only effect contributing to the overall actuation mechanism. It is believed that this response is a result of contributions from electrostatic, elastic, polaronic, and thermal effects that cause this impressive overall photo-response of GNP/PDMS composites similar to CNT/PDMS composites $^{51,52,62}$.

Exploring GNP/PDMS composites further, questions arise as to why GNP/PDMS performs better than the other carbon counterparts tested. While it is understandable GNPs performed better than $\mathrm{CB}$ and GO/PDMS mixtures, questions linger as to the reason for smaller amplitudes of photo-mechanical stress for CNT/PDMS. There are 


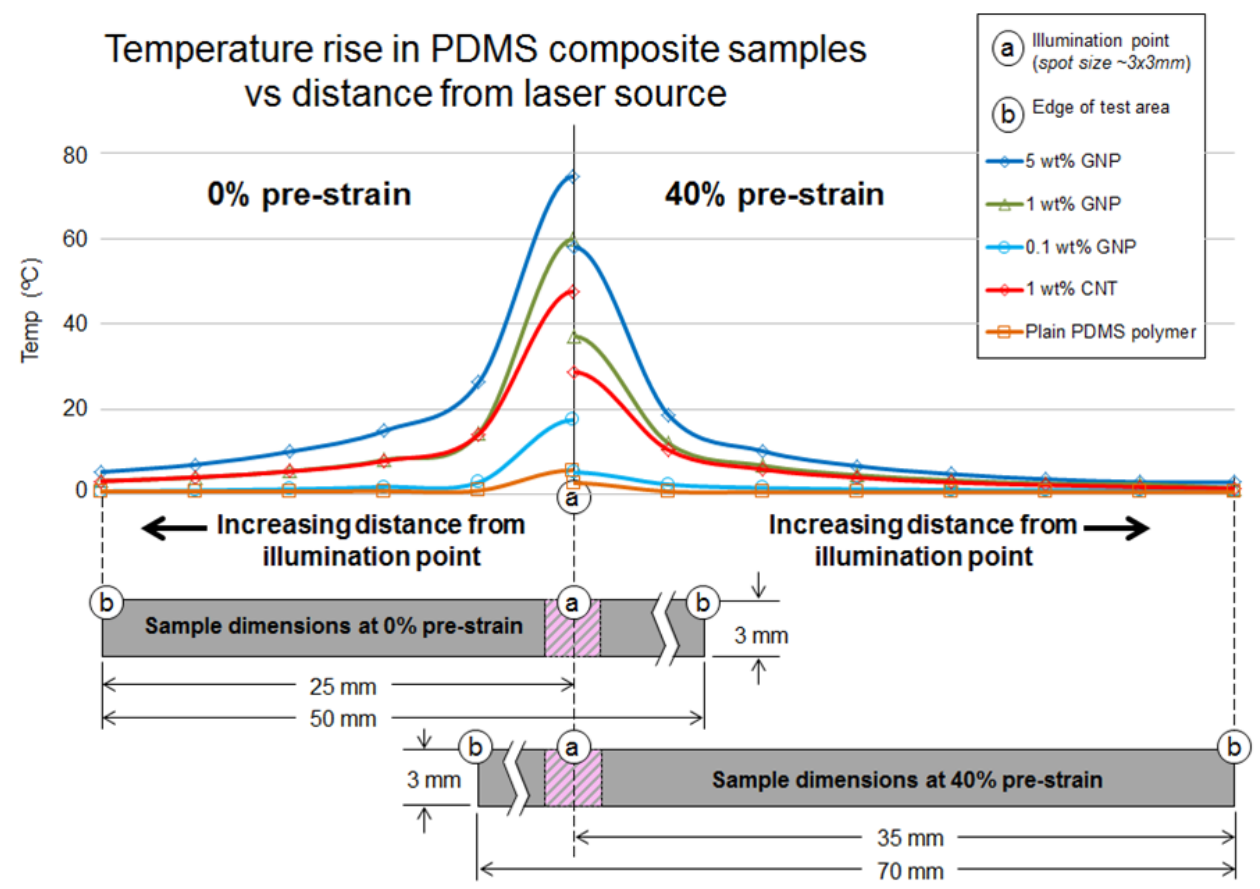

Figure 3.6. Steady-state temperature measurements. Temperature decrease along the composites test samples as a result of distance from the illumination point. $0 \%$ pre-strain dimension and temperature response (left side) are compared with $40 \%$ pre-strain dimensions and temperature response (right side).

several likely reasons for this which warrants further research. First, the 2D nature of GNPs enables sliding of individual platelets on top of one another and thus resulted in homogenous sample dispersions within a relatively short time ( $\sim 5$ minutes $)$ shear mixing process. GNPs act like discrete $2 \mathrm{D}$ rigid plates. Due to similar atomic arrangement of carbon and crystal-like behavior, friction and interaction effects between the graphene platelets is expected to be negligible. However in CNT/PDMS composites, the short time shear mixing was not long enough to overcome the natural tendency of nanotubes to form bundles due to their enhanced inter-tube interactions, thus giving rise to smaller amplitudes of photo-mechanical stress. In other words, nanotubes exhibited "particle- 
like" behavior for short mixing times rather than that of a matrix of dispersed 1D tubes. Hence the photo-mechanical stresses exhibited by CNT/PDMS actuators were quite similar to $\mathrm{CB} / \mathrm{PDMS}$ actuators for the short mixing time. Therefore, both matric orientation and percolation threshold is crucial for enabling high amplitudes of photomechanical stress and faster kinetics for CNT/PDMS actuators ${ }^{64}$. Longer mixing times and more sophisticated dispersion methods are needed to separate the nanotubes from the bundles as well as rendering them oriented in the PDMS matrix.

The maximum photo-mechanical stress change reported here corresponds to an optical-to-mechanical energy conversion factor, $\eta_{\mathrm{M}}$ of 7-9 MPa/W. This conversion factor is significantly higher than for commercial polymers, such as polyvinylidene fluoride (PVDF) whose calculated $\eta_{\mathrm{M}}$ value of $\sim 97 \mathrm{kPa} / \mathrm{W}^{193}$, and azobenzene containing polymers and gels ${ }^{194}$. While rapid photo-mechanical actuation has been attained in azobenzene containing polymers ${ }^{195}$, practical applications are difficult due to dependency on precise location of the azobenzene moieties in dark as well as photo-plastic effects making it relax slowly, in $\sim 10$ minutes ${ }^{196}$. On the other hand, shape memory (SM) polymers are quite interesting. When SM polymers are elongated up to $20 \%$ followed by irradiation of the actuator at $\lambda=260 \mathrm{~nm}$ for 60 minutes, this process releases the stress and causes relaxation ${ }^{169}$. Such prolonged stress relaxation, however, could lead to very long actuation time that is not practical for many commercial applications. Recently, LCEs have emerged as interesting polymers due to the polymeric network and liquid crystal anisotropic chain. Thermo-responsive LCEs have shown large contractions of 35$40 \%$ throughout the phase transition with a force of $0.001 \mathrm{MPa} /{ }^{\circ} \mathrm{C}$, comparable to that of skeletal muscle ${ }^{197}$. Similarly ferro-electric LCE films have shown $4 \%$ strains at 
1.5 MV/cm, making them expensive with respect to energy consumption ${ }^{42}$. Compared to these past reported actuators with impressive characteristics, the reversible contraction and expansion in GNP/PDMS provides fast actuation and relaxation; furthermore, actuation is elastic and samples actuate during light on and return to the original state within $5 \mathrm{~s}$ after light off. While past nanotube/PDMS composites have exhibited similar stresses as GNP/PDMS composites reported here ${ }^{62}$ for different fabrication recipes, nevertheless, GNPs are less expensive and far easier to disperse compared to CNTs in polymer matrix. With a high energy conversion ratio of 7-9 MPa/W, GNP-based materials could be one of the best material choices for future mechanical actuators, thermo-electric based devices, and solar energy converters.

\subsection{CONCLUSIONS}

This chapter reports large light-induced reversible and elastic responses of graphene-based/polymer composites. Homogeneous mixtures of GNP/PDMS composites $(0.1-5 \mathrm{wt} \%)$ were prepared and their IR mechanical responses studied with increasing pre-strains. Using IR illumination, a photo-mechanically induced change in stress of four orders of magnitude as compared to pristine PDMS polymer was measured. Actuation responses of graphene polymer composites depended on applied pre-strains. At low levels of pre-strains (3-9\%) the actuators showed reversible expansion while at high levels (15-40\%) the actuators exhibited reversible contraction. GNP/PDMS composites exhibited higher actuation stresses compared to other forms of nanostructured carbon/PDMS composites, including CNTs, for the same fabrication method. An extraordinary optical-to-mechanical energy conversion factor $\left(\eta_{\mathrm{M}}\right)$ of 7-9 MPa/W for GNP-based polymer composite actuators is reported. 
This study demonstrated the intrinsic photo-mechanical actuation of graphenebased polymer composites. Actuation is witnessed only through the presence of graphene. Dispersion of GNPs into a PDMS silicone elastomer matrix resulted in a novel GNP-based photo-mechanical actuator that showed reversible light-induced elastic expansion and contraction. For a $2 \mathrm{wt} \%$ sample, a photo-mechanically induced change in stress of $+14 \mathrm{kPa}$ at low pre-strains, and $-36 \mathrm{kPa}$ at high pre-strains was successfully demonstrated. With an optical-to-mechanical energy conversion factor of $\eta_{\mathrm{m}} \sim 7-9 \mathrm{MPa} / \mathrm{W}, \mathrm{GNP} / \mathrm{PDMS}$ composites have outperformed most photo-responsive materials, including all other carbon forms tested for the fabrication method described here. Looking forward into a variety of possible future applications of photo-mechanical actuators, one can develop surfaces that can undergo simultaneous expansion and contraction. On a small scale, this could be used for assembling surfaces to study mechanical forces on soft matter, such as biological materials such as cells, where the forces are delivered in non-contact manner using photo-mechanical actuation. On a large scale, thin films of GNP/PDMS polymer could be utilized as a fundamental smart material in adaptive skins. The temperature decrease with increase in pre-strains of the actuators could be useful for developing a new type of strain gauge owning to linear temperature change with strain. In the field of sensing, graphene based photo-mechanical actuators could be highly useful for sensing adsorbed molecules that result in a change of the actuator's stress state. Finally, the distinct stress response states of the photo-actuators (positive, zero and negative) could be useful for designing and nano-mechanical memory. Instead of a cantilever gently touching the surface of the polymer pit to write, it could be stressed simultaneously at millions of points using single light source enabling nanoscale 
photo-mechanical writing. Such photo-mechanical writing could be reversible and fast, as well as actuated through controlling the number of photons reaching the surface.

While these GNP/elastomer composite systems demonstrate unique photomechanical actuation characteristics; thus making them interesting systems to study, it was not clear whether the number of graphitic layers contained within each the carbon nanostructure had a bearing on overall photo-mechanical response. To investigate these composite systems further, therefore, layer-dependent properties were studied, forming the basis of the next chapter. 


\section{CHAPTER 4 \\ LAYER DEPENDENT MECHANICAL RESPONSES OF GRAPHENE COMPOSITES TO NEAR-INFRARED LIGHT}

\subsection{INTRODUCTION}

While investigation into use of few layer graphene/polymeric composites (comprised of GNPs, $\sim 5$ layers) as photo-mechanical actuators was shown to demonstrate an impressive optical-to-mechanical energy conversion of $\sim 7 \mathrm{MPa} / \mathrm{W}^{54}$, many questions remain regarding the nature of photo-mechanical effects in graphene polymer composites. Photo-responsive difference between multi versus single layer graphitic crystal polymer composites is unknown. Optical absorption of graphene is $\sim 2.3 \%$ per layer over the visible spectrum ${ }^{72}$. Rigid stack-like morphology and lack of inter-plate interactions of GNPs could mean that their dispersion properties and eventual photomechanical response could be different in polymer composites compared to single layer graphene.

\subsection{RESULTS}

This chapter reports photo-mechanical responses in composites comprised of small quantities $(0.01-1 \mathrm{wt} \%)$ of SLG dispersed within PDMS, a widely available commercial polymer. To evaluate layer dependency of responses, GNP/PDMS composites were fabricated and used as a basis for comparison. SLG was prepared through complete reduction of graphite oxide using modified Hummer's 
method. GNPs were synthesized using plasma exfoliation of high purity graphite. Both forms of graphene were studied extensively with scanning electron microscopy and Raman spectroscopy to verify number of layers prior to fabrication. Figures 4.1(a)-(b) show SEM images of SLG sheet and GNP stack, respectively. The dramatic difference in morphologies between the two graphitic materials is due to just few atomic layers of carbon. Figure 4.1(c) compares layer dependent shifts of $G$ and $2 D$ Raman peaks. Multilayer configuration of GNPs causes a frequency shift in both peaks as compared to SLG from past reports ${ }^{198}$.

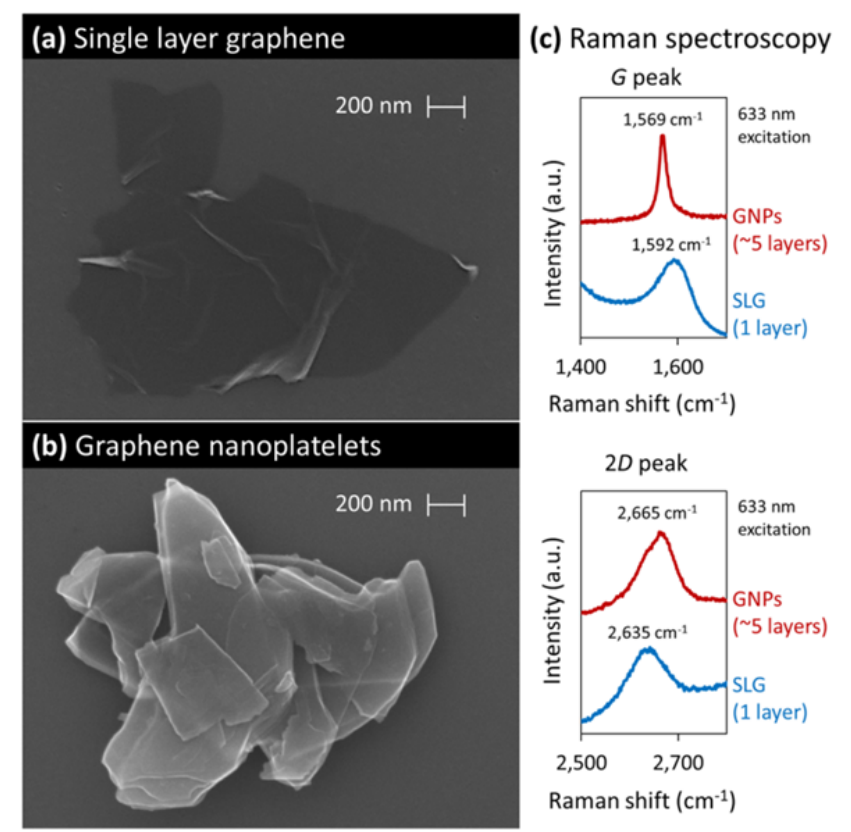

Figure 4.1. SEM and Raman carbon characterization. (a) SEM image of pristine SLG sheet on silicon wafer. (b) SEM image of pristine GNP stack on silicon wafer. (c) Layer dependent shift of $G$ and $2 D$ peaks at $633 \mathrm{~nm}$ excitation.

A new fabrication procedure was developed to homogeneously disperse SLG within PDMS. Figure 4.2(a) provides a sequence overview involving sonication of 
SLG/FLG in isopropyl alcohol (IPA), evaporative mixing, crosslinking, degas procedures, and final actuator fabrication using spin coating. Commercially obtained SLG ( $\sim 92 \%$ carbon, $<8 \%$ oxygen, produced via thermal exfoliation reduction and hydrogen reduction of single layer graphene oxide) was purchased from ACS Materials. Appendix B provides sample preparation steps. Test samples were mounted between test dynamometer upper and lower clamp assemblies [Figure 4.2(b)]. Testing was completed as in $\operatorname{Ref}[54]$.

(a) Evaporative mixing fabrication

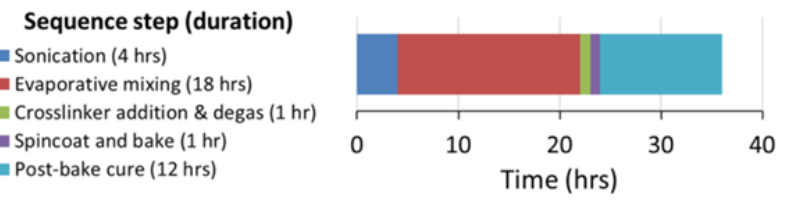

(b)

Test dynamometer

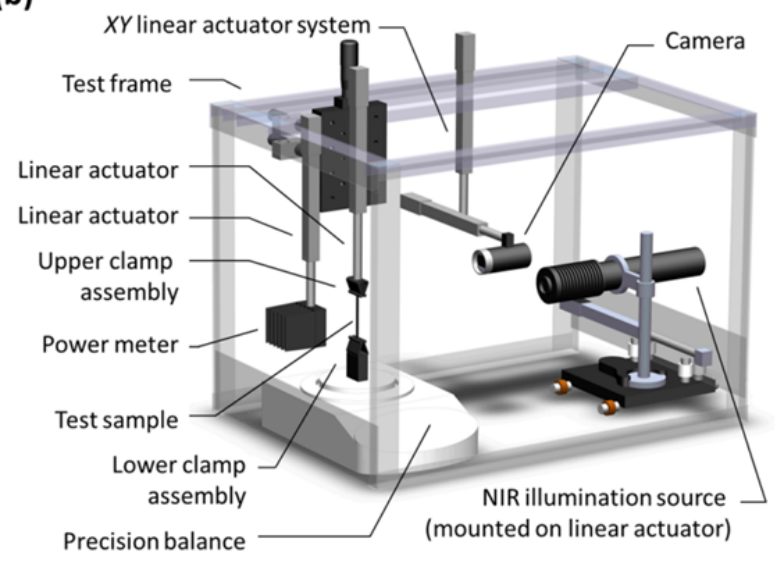

Figure 4.2. Composite fabrication and test setup. (a) Evaporative mixing fabrication sequence. (b) Layout of test dynamometer.

Figure 4.3(a) presents the photo-kinetic response during a test of $1 \mathrm{wt} \%$ SLG/PDMS composite at $3 \%$ pre-strain. A $+17 \mathrm{kPa}$ change in actuator stress is clearly observed. Both actuation and relaxation of the SLG/PDMS polymer were found to follow simple exponential function $\exp [-(t / \tau)]$ with $\tau \approx 1.5 \mathrm{~s}$, following Debye relaxation for 
polymers. Figure 4.3(b) presents the increase in Young's modulus, $E$, starting with $\sim 1.6 \mathrm{MPa}$ for pristine polymer and increasing $25 \%$ to $2 \mathrm{MPa}$ in $1 \mathrm{wt} \%$ of SLG. Using forecasting equation derived from experimental data, $E$ is expected to double at an impressively low $\sim 5 \mathrm{wt} \%$ SLG loading. Even the $25 \%$ increase observed within the experimental regime is remarkable, however, considering the low SLG concentration. Past results on GNP actuators report a $\sim 60 \%$ rise in $E$ for $5 \mathrm{wt} \%$ loading, thus showing a layer dependent effect ${ }^{54}$. Figure $4.3(\mathrm{c})$ presents change in stress of $1 \mathrm{wt} \% \mathrm{SLG} / \mathrm{PDMS}$ as a function of increasing pre-strains and shows distinct response states. At low pre-strains $(\sim 3-9 \%)$ actuators expand, with maximum observed change in stress of $\sim 17 \mathrm{kPa}(1 \mathrm{wt} \%$ SLG). At moderate pre-strains ( $10-15 \%)$, actuators have negligible stress response. At high pre-strains ( $>15 \%$ ) actuators contract, exhibiting maximum value of $\sim 32 \mathrm{kPa}$ ( $1 \mathrm{wt} \%$ SLG). Repeatability over 24-hr period of cycling near-infrared (NIR) on/off was measured and found to be stable in expansive and contractive modes. Stability suggests minimal degradation of polymer matrix due to temperature dependent viscoelastic effects. As pre-strain increases, induced orientational effects cause SLG sheets within the polymer to become more aligned with respect to one another, thus resulting in photo-mechanical response amplification. Maximum total observed change in stress from expansion to contraction in $1 \mathrm{wt} \% \mathrm{SLG} / \mathrm{PDMS}$ was $\sim 50 \mathrm{kPa}$. Translated into a stroke, this corresponds from $\sim 0.8 \%$ expansion to $\sim 1.6 \%$ contraction. 

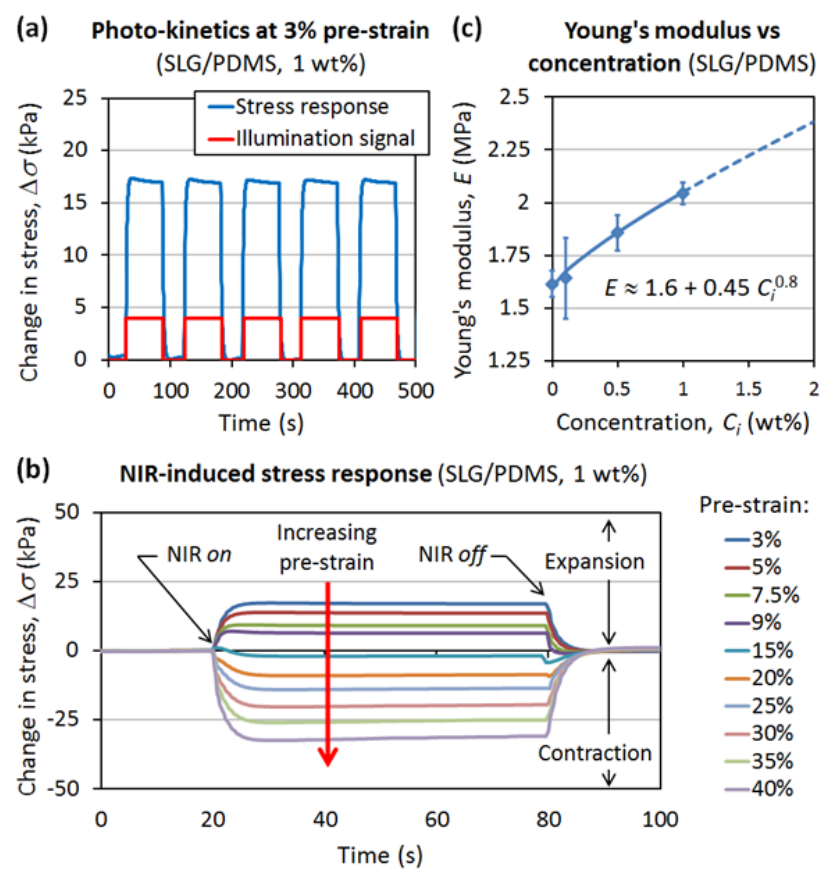

Figure 4.3. SLG/PDMS photo-mechanical stress responses and Young's modulus. (a) Photo-kinetics of SLG/PDMS at 3\% applied pre-strain showing standard 5-cycle test run as well as actuation and relaxation kinetics. (b) Young's modulus as a function of increasing SLG loading. (c) Detail of single NIR stress cycle for $1 \mathrm{wt} \%$ SLG/PDMS at applied pre-strains from 3-40\% pre-strain with NIR off stress for all pre-strains normalized to zero.

Figure 4.4(a) presents relative change in stress (from 3-40\% pre-strain) of SLG and GNP composites as function of both $w t \%$ concentration and NIR laser power. Across the board, SLG-based composites exhibit more than twice the recoverable stress compared to their multilayered GNP counterparts. With respect to concentration, upward correlation between increasing stress response with increasing $\mathrm{wt} \%$ carbon loading was observed. As opposed to upward trend with concentration, above $\sim 50 \mathrm{~mW} / \mathrm{mm}^{2}$ SLG/PDMS composites exhibited saturable mechanical response. For identical wt $\%$ 
loading and NIR intensity, stress response was seen to decrease $\sim 20 \%$ with each additional layer of graphene. Next, optical-to-mechanical energy conversion efficiencies were calculated based on stored potential energy to incident light energy, following methodology in Ref [199]. Figure 4.4(b) presents optical-to-mechanical energy conversion efficiency as function of both concentration and pre-strain. Efficiency increased linearly with concentration for both SLG and GNP/PDMS composites. Note that laser spot size was only $3 \times 3 \mathrm{~mm}$. As entire test sample was 50 by $3 \mathrm{~mm}$, not only was just $6 \%$ exposed to NIR illumination, but graphene contained in this small section was also responsible for the entire photo-mechanical response. Higher responses and efficiencies can be obtained by increasing illumination to sample area ratio. Finally, efficiency with respect to pre-strain for SLG/PDMS was evaluated. Zero efficiency value merely indicates balance between pre-strain and photo-mechanical forces. As actuators changed state from expansion to contraction ( $>15 \%$ pre-strain), however, magnitude of stress response, and therefore efficiency, increased as well. Again, layer dependency on efficiency was witnessed, with each additional graphene layer lowering efficiency $\sim 30 \%$. Decrease in stress as well as efficiency with number of layers depend on few factors such as optical absorption, surface area increase for SLG versus decrease for stack-like GNPs in contact with the polymer, "rigid rod-like" (FLG) versus "flexible ribbon-like" (SLG) behavior affecting overall dispersion, and finally percolation threshold. Lower photomechanical response of multilayer composites may be due to the relationship of decreasing surface area to volume for polymer interaction with increasing number of layers. The GNP and SLG should have same amount of carbon content for same wt $\%$ loading. Photo-mechanical response is determined by amount of carbon in contact with 
polymer. SLG can weave itself within the polymer creating a continuous matrix, while GNPs with rigid stacked sheet-like morphology cannot; and effective surface area for photon absorption, elastic modulus, and actuation therefore decreases. Due to this, GNPs may only use a fraction of absorbed energy to create localized heating causing expansion or contraction of polymeric chains. In other words, for the same $\mathrm{wt} \%$ loading, increasing each additional graphene layer loses a layer of contact with the polymer.

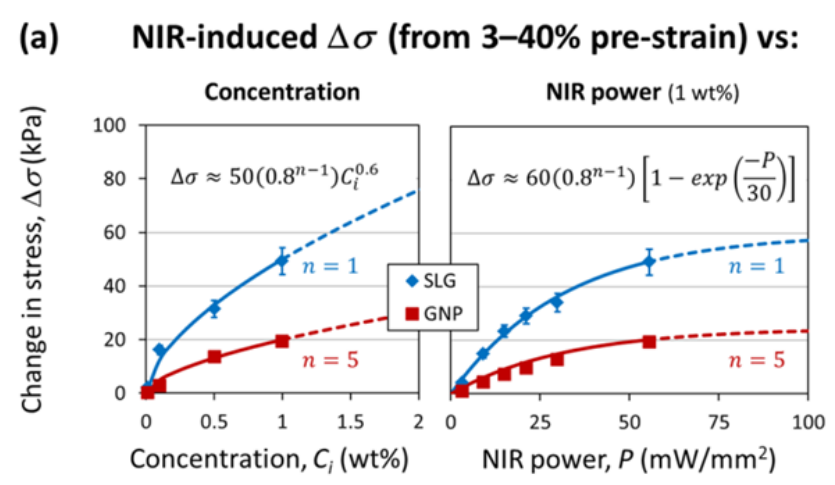

(b) NIR-induced efficiency vs:

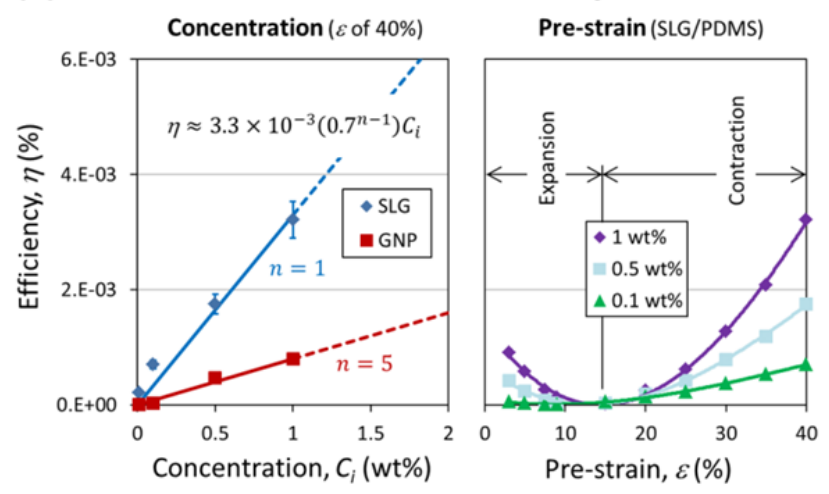

Figure 4.4. NIR-induced stress and efficiency comparison between SLG and GNP-based composites. (a) Stress dependency as a function of both concentration and laser power for a fixed wt\%. (b) Efficiency vs concentration and pre-strain. 


\subsection{CONCLUSIONS}

In summary, layer dependent physical responses of graphene-based polymer actuators are reported, with FLG and SLG composites tested to determine photomechanical responses. Layer dependent stress and efficiency responses were witnessed, with a decrease of $20 \%$ and $30 \%$, respectively, per additional graphene layer. These results suggest that dispersion of number of graphene layers in contact with polymer is a critical consideration in designing graphene-based composites for mechanical strength, ultrafast photonics, optoelectronics, and energy conversion devices.

While this chapter demonstrated a direct correlation between number of graphitic layers contained in the carbon nanostructures and the resulting photo-mechanical and efficiency responses, another question arises - what role does the dimensional state of the carbon nanostructure play in photo-response? As carbon's dimensional state is known to affect heat transfer and mechanical strength, the following chapter presents a study of the optically-induced actuation responses in four different (although chemically identical) carbon nanostructure-based composites, ranging from 1D CNTs to 3D HOPG. 


\section{CHAPTER 5 \\ DIMENSIONAL DEPENDENCE OF PHOTO-MECHANICAL RESPONSE}

\subsection{INTRODUCTION}

Having investigated layer dependent effects in the previous section, this chapter investigates photo-mechanical responses to near-infrared wavelengths of light in four different dimensional types of synthetic carbon nanostructure/PDMS composites with concentrations from 0.01 to $1 \mathrm{wt} \%$; including MWNT/PDMS (1D), SLG/PDMS (2D), GNP/PDMS (2.5D), and highly ordered pyrolytic graphite (HOPG)/PDMS (3D). A dispersion method using slow evaporative mixing of nanostructured carbon in PDMS was developed to completely homogenize carbon within the final composite. Intriguingly, results show direct correlation between increasing photo-mechanical stress and efficiencies with reducing dimensionality of carbon nanostructures in the composites. Extraordinary opto-mechanical energy conversion efficiencies of up to $\sim 5 \times 10^{-3} \%$ were obtained, despite low carbon nanostructure concentrations (0.01 to $1 \mathrm{wt} \%)$. Additionally, actuation and relaxation kinetics were found to be related to the relationship of carbon additive to the percolation threshold (PT). These intriguing results provide a foundation for carbon-based micro- and nano-opto-mechanical systems, where dimensional state of the carbon nanostructure can be exploited for use in advanced photonic and optoelectronic devices, photo-mechanical sensors, and actuator applications. 


\subsection{RESULTS}

Figures 5.1(a)-(d) show scanning electron microscope (SEM) images of MWNTs, SLG, GNPs, and HOPG deposited on a silicon wafer. The arrangement of MWNTs [Figure 5.1(a)] with their 1D structure is that of nested, entangled, high aspect ratio tubes. Figure 5.1(b) shows a wrinkled SLG sheet lying on the substrate. Wrinkles provide thermodynamic stability for the 2D sheet of carbon atoms, giving structural integrity and preventing self-collapse ${ }^{103}$. As feature height increases in 2.5D GNPs [Figure 5.1(c)], stacks of disoriented plates are observed. The distinct contrasting morphology between SLG and GNP is remarkable given that the difference is due to just a few atomic layers of carbon. GNPs appear as rigid, partially transparent plates whereas SLG more resembles a draped silk sheet. Finally, true 3D features become apparent in HOPG nanostructures

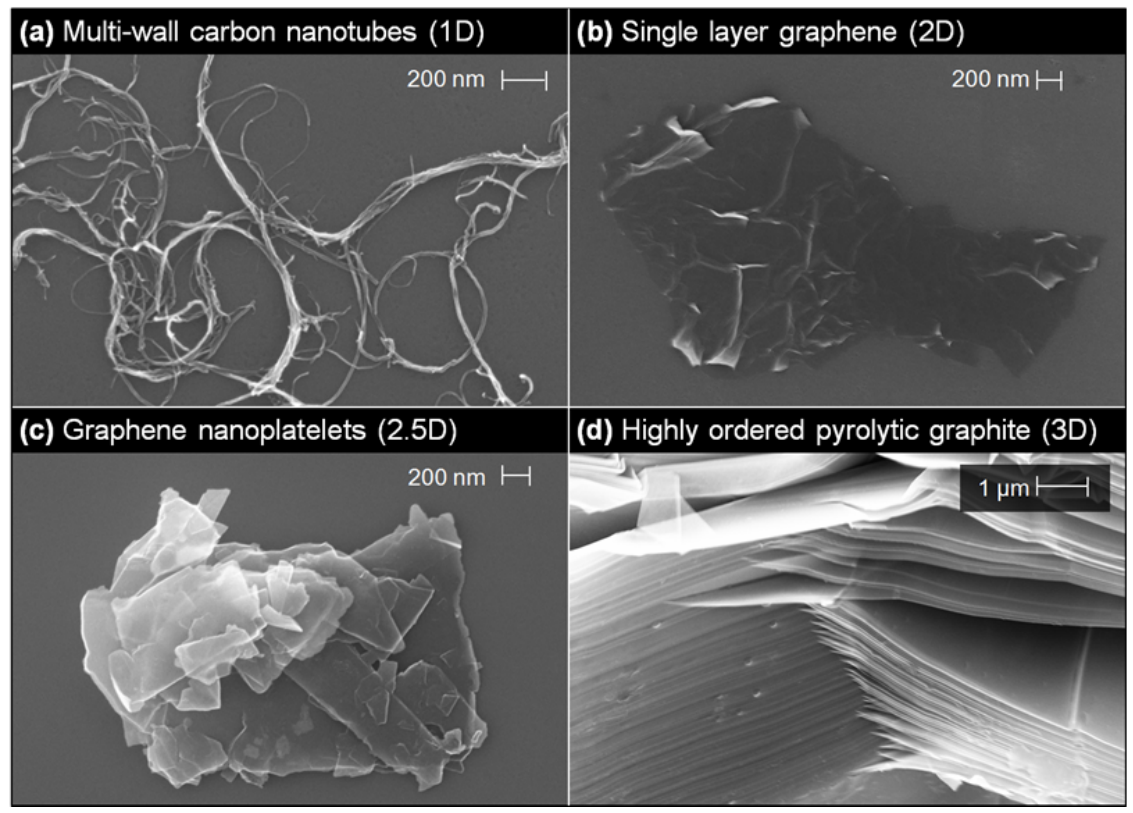

Figure 5.1. Carbon additive characterization. (a) SEM of MWNT, a 1D material. (b) SEM of SLG, a 2D material. (c) SEM of GNP, a 2.5D material. (d) SEM of HOPG, a 3D material. 
[Figure 5.1(d)], whose SEM image shows hundreds of layers stacked on top of one another like sheets of paper. Raw carbon additive purity was confirmed via Raman spectroscopy prior to composite fabrication ${ }^{53,54}$. The $G$-band (graphite) and $G^{\prime}$-bands were evaluated as they are known to shift position, shape, and intensity with respect to the number of layers ${ }^{198}$. The $G$-band decreased in frequency with increasing layers, following a $1 / n$ dependence ${ }^{200}$. The $G^{\prime}$-band exhibited more complex behavior than the $G$-band, shifting from just a single Lorentzian peak (graphene), up to four peaks (bilayer graphene), and then finally just two Lorentzian peaks (graphite) ${ }^{200}$.

Following extensive SEM and Raman characterization, dispersion of the carbon nanostructures in PDMS was the next step towards realizing photo-mechanical actuators. Sample homogeneity and carbon nanostructures dispersion state are critical factors affecting photo-mechanical response. For example, past reports on SWNTs have demonstrated that homogenous distribution is crucial in eventual photo-mechanical response $^{54}$. For purely shear mixed dispersion methods, homogenization of carbon nanostructure additive depends on mixing time. For short time shear mixed fabrication methods, some carbon nanostructure additives (such as CNTs) can act as particles rather than dispersed additives, resulting in suppression of photo-mechanical response, as seen in past reports ${ }^{54}$. Therefore, a fabrication method that utilized slow evaporative mixing (between the carbon additive, IPA and PDMS base compound) over 18 hours was developed to homogeneously distribute difficult-to-disperse additives ${ }^{53}$ (see Appendix B). Carbon nanostructure additive was first sonicated in IPA, then transferred to PDMS base compound through evaporative mixing, followed by cross linker addition 
and finally spin casting to produce homogeneous flexible thin films $(60 \mu \mathrm{m})$. Once these composite films were fabricated, $3 \mathrm{~mm} \times 60 \mathrm{~mm}$ samples were cut for testing.

Figure 5.2(a) presents an experimental test dynamometer setup schematic showing vertical test sample orientation. The entire setup was isolated inside a robotic black box and controlled remotely through a host computer, thus eliminating any mechanical response due to ambient light. Figure 5.2(b) shows a video capture of a carbon/PDMS sample undergoing testing. The test sample (circled) was mounted between the upper and lower clamp assemblies with the $\sim 3 \mathrm{~mm} \times 3 \mathrm{~mm}$ NIR illumination point clearly visible at the sample vertical center. In order to demonstrate differences between local and global photo-mechanical responses, which are discussed later in the chapter, only a fraction of the overall test sample was selectively illuminated. A power meter behind the test strip measured light transmitted through the sample, allowing calculation of light absorbed by subtracting the power meter reading from raw laser power. Due to practical difficulties accurately measuring Young's modulus $(E)$ using conventional methods, values of $E$ were calculated from the stress induced in the samples as a result of pre-straining. Elastomers have the ability to sustain large deformations with essentially complete recovery $^{201}$, therefore Young's moduli values for each carbon type and concentration were determined using $E=\sigma_{\mathrm{NIR}}$ off $/ \varepsilon$ for each pre-strain, and an overall $E$ determined by averaging the individual values. Young's modulus of PDMS is widely tunable, as a function of several factors, including ratio of base compound to crosslinker $^{202}$, baking time and temperature ${ }^{203}$, and amount as well as type of additives to the polymer $^{53,54}$. Past graphitic carbon reports project a doubling of $E$ at relatively low carbon loading of $\sim 5 \% \mathrm{SLG}^{53}$. 
(a) Test dynamometer

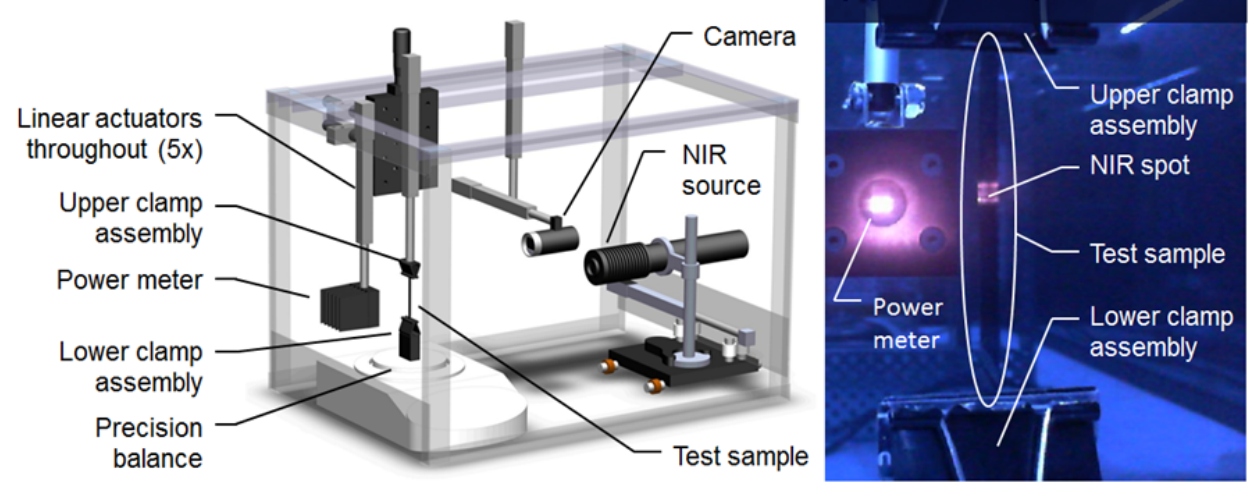

Figure 5.2. Carbon/PDMS photo-mechanical actuator fabrication and testing. (a) Layout of test dynamometer. (b) Video capture of test sample undergoing NIR illumination.

\subsubsection{STRESS RESPONSE COMPARISON}

Figure 5.3(a) (left side) shows a compilation of stress responses during a single 60 s NIR on/off cycle over the entire range of pre-strains for $1 \mathrm{wt} \%$ SLG/PDMS. At $20 \mathrm{~s}$, NIR illumination was switched on, and photo-mechanical stress responses recorded. At $80 \mathrm{~s}$, NIR illumination was turned off and the perturbed samples relaxed to their pre-strain equilibrium stress. As shown by the absolute stress response graph, prior to NIR illumination, a stress $\left(\sigma_{\mathrm{NIR}}\right.$ off $)$ was induced as a result of pre-strain. The relative NIR-induced stress [Figure 5.3(a), right side] more clearly illustrates the three distinct stress response states exhibited by all carbon/polymer composites At low pre-strains $(\sim 3-9 \%)$ composites expanded, moderate pre-strains $(10-15 \%)$ resulted in minimal stress response change, and finally, high pre-strains $(>15 \%)$ resulted in sample contraction.

Figure 5.3(b) shows sample stability in long-term response testing in both expansive and contractive modes over the course of $24 \mathrm{~h}(\sim 1,000$ cycles $)$ for $1 \mathrm{wt} \% \mathrm{SLG} / \mathrm{PDMS}$. The two inset graphs show details of individual cycles, while only the magnitude of the stress response change is shown on the larger graph. The stability of the actuator both in 
(a) Absolute and relative NIR-induced stress response (1 wt\% SLG/PDMS)
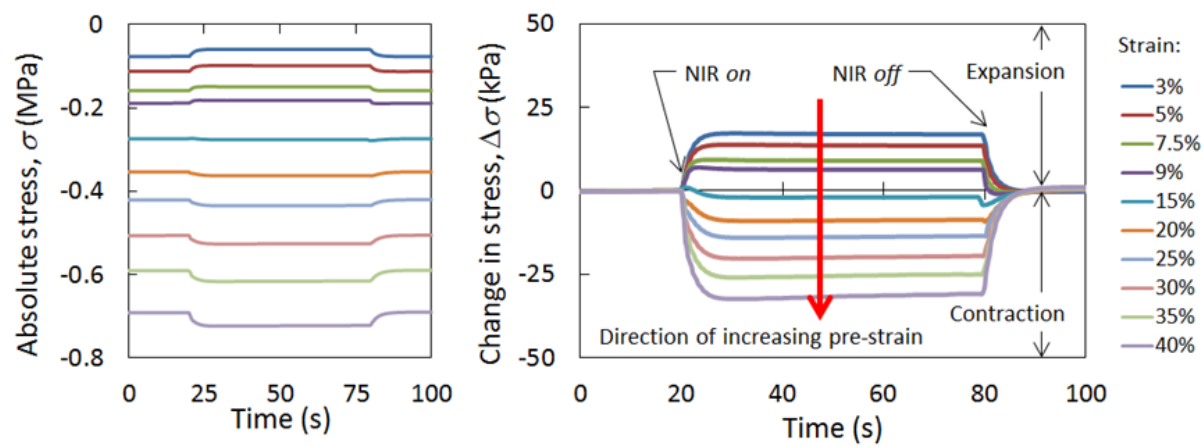

(b) Long-term NIR-induced stress response (1 wt\% SLG/PDMS)

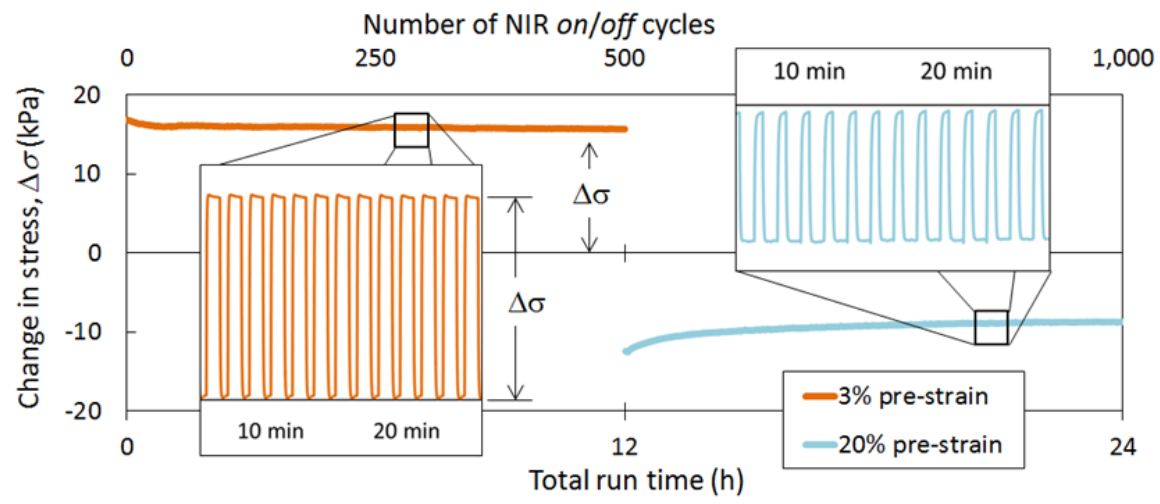

Figure 5.3. Stress response and long-term characterization for SLG/PDMS. (a) Absolute and relative NIR-induced stress responses of SLG/PDMS. (b) Long-term steady-state cycling response showing stability of the photo-mechanical actuator both in expansive and contractive actuation modes over $24 \mathrm{~h}$.

expansive and contractive modes suggests minimal nonlinear viscoelastic effects, thereby maintaining original polymer configuration ${ }^{62}$. Appendix $\mathrm{C}$ details the testing sequence.

\subsubsection{INCREASING STRESS RESPONSE AS A FUNCTION OF DECREASING DIMENSIONALITY}

Next, effects of photo-mechanical response and actuation kinetics as a function of dimensionality of carbon nanostructures were investigated. Figure 5.4(a) illustrates the relationship between steady-state photo-mechanical actuation and carbon dimensional 
(a) Increasing photo-induced stress with decreasing dimensions of carbon/PDMS composites from 3-40\% pre-strain (1 wt\%)

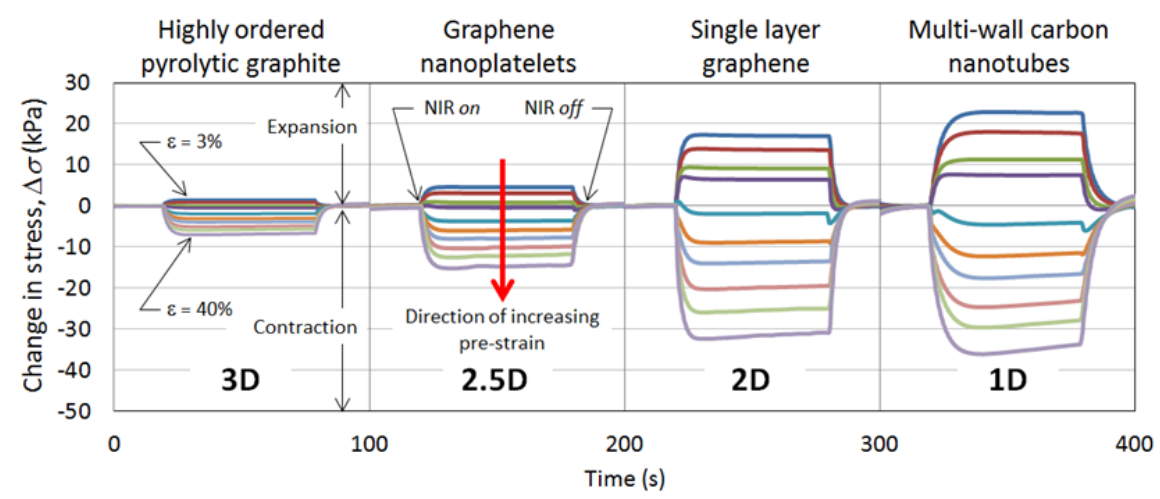

(b) Photo-kinetics of carbon/PDMS composites (1 wt\%)

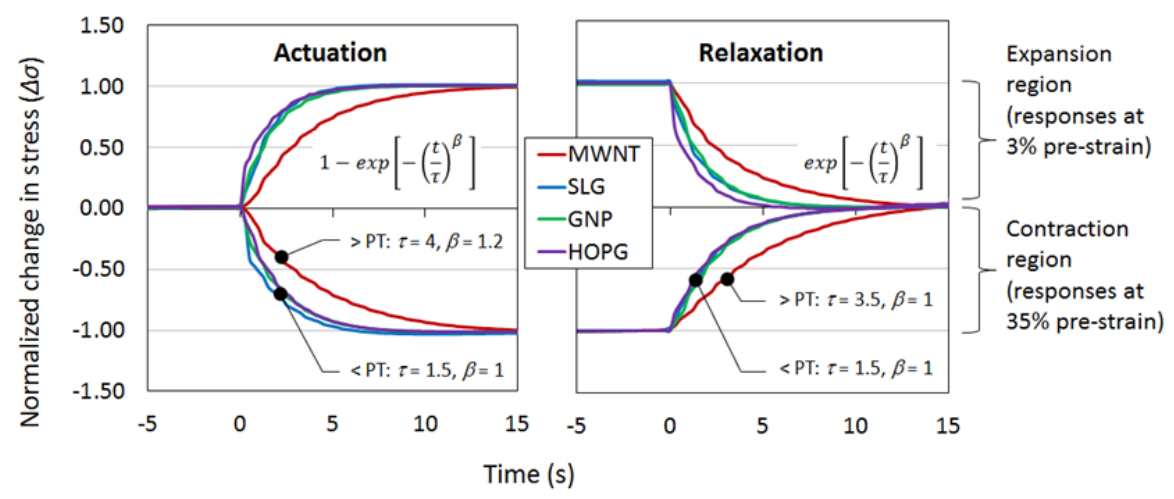

Figure 5.4. Comparison of increasing photo-mechanically induced stress change with decreasing carbon dimensionality. (a) Photo-mechanical stress response for $3-40 \%$ prestrains starting with a 3D carbon composite (HOPG/PDMS) and progressing to a $1 \mathrm{D}$ composite (MWNT/PDMS) due to NIR illumination. (b) Photo-kinetics of $1 \mathrm{wt} \%$ carbon/PDMS composites showing 3\% and 35\% pre-strain actuation and relaxation kinetics dependent on the relationship to the PT.

state. Starting with a 3D carbon additive (HOPG), and dropping to a $1 \mathrm{D}$ additive (MWNT), the plots show photo-mechanical response from 3-40\% pre-strains. An intriguing result was observed; as dimensionality of carbon nanostructure additive in PDMS polymer decreases, NIR-induced photo-mechanical stress response increases. MWNT-based (1D) composite witnessed the maximum stress response seen in any of the 
composites, followed by SLG (2D), GNP (2.5D) and HOPG (3D) respectively. Upon NIR illumination, a number of events occur before final photo-mechanical response is achieved. These include photon absorption by carbon nanostructure additive and subsequent energy transduction to the polymeric chains, resulting in expansion or contraction of the polymer (depending on applied pre-strain). No stress response was seen in pure PDMS samples upon NIR irradiation, thus photo-mechanical response is due to the presence of each type of carbon nanostructure in the composite ${ }^{54}$.

Figure 5.4(b) details actuation and relaxation photo-kinetics. In order to show expansive as well as contractive modes, normalized $3 \%$ and $35 \%$ pre-strains curves for each carbon nanostructure were plotted. Interestingly, rather than a dependence on dimensionality, kinetics were found to be related to PT. Composite PT is a critical value at which conductive (carbon) additives within an insulating host matrix (PDMS) result in the composite becoming electrically conductive ${ }^{204}$. Crossing this threshold is accompanied by a resistance drop of several orders of magnitude. Curves were fit to experimental actuation and relaxation data by incorporating the Kohlrausch-WilliamsWatts function ${ }^{205}$ as $\Delta \sigma_{\text {actuate }}(t)=1-\exp \left[-(t / \tau)^{\beta}\right]$ and $\Delta \sigma_{\text {relax }}(t)=\exp \left[-(t / \tau)^{\beta}\right]$, respectively. Regardless of dimensionality, responses for carbon nanostructure composites at loadings less than the PT were found to be nearly identical and distinctly different than those for loadings greater than the PT. At $1.5 \mathrm{~s}$ for both actuation and relaxation, the time constant $(\tau)$ for carbons less than the PT was much quicker than that for carbon greater than the PT (3.5-4 s). As evidenced by lack of conductive network, quicker response times indicate that the photo-mechanical effect is localized to the area of NIR illumination. A longer actuation time, as given in the 1D system, indicates photo- 
mechanical energy conversion response is spreading throughout the actuator (beyond the illumination point), and thus requiring longer time to reach equilibrium. As PT is breeched, photo-mechanical response not only occurs at the NIR illumination point, but also bleeds to the surrounding areas where carbon has established a connective network. Therefore, by crossing PT, photo-mechanical energy conversion and subsequent mechanical responses change from localized to global effects, resulting in amplified response due to more activated carbon. Selectively illuminating only a portion of the strip helps highlight the increase in stress with change from local to global responses. Different relaxation times between carbons less than and greater than PT are different from past reports, which recount identical relaxation times regardless of loading ${ }^{62}$. Further evidence that photo-mechanical energy transduction is spreading beyond the area of NIR illumination is given by the stretching exponent $(\beta)$. Carbon additives less than the PT follow classical polymer Debye kinetics $(\beta=1)$. For carbons tested greater than PT, $\beta=1.2$ for actuation, indicating an influence by the carbon network outside the NIR illumination point. For the methods used in these experiments, the only carbon nanostructure to establish a conductive network by $1 \mathrm{wt} \%$ was MWNT/PDMS. It is believed that the fibrous nature of the MWNTs allowed formation of an electrical network at much lower concentrations than the thick stacked paper-like HOPG, plate-like GNPs, or sheet-like SLG. While further research is necessary, it is expected that for a given fabrication method, PT also follows a dimensional relationship, where 1D nanostructures can establish a PT at lower wt\%'s compared to their 2D and 3D counterparts. Rather than being a set value, PT is dependent on a variety of factors including dimension of the carbon nanostructure (amount of anisotropy) ${ }^{206,207}$, type of 
carbon (SWNT, MWNT, etc.) in the polymer ${ }^{208}$, and dispersion method ${ }^{209}$. An assortment of PT values for CNTs have been reported, ranging from 0.0025 to $4 \mathrm{wt}^{\mathrm{0}} \mathrm{o}^{209,210}$.

Therefore, it is expected that PTs for graphene will depend on factors such as number of layers, length and width of the sheets and plates, and finally the amount of carbon in contact with the polymer for both SLG and GNP ${ }^{53}$.

\subsection{CONDUCTIVE COMPOSITE PHOTO-MECHANICAL RESPONSE}

Further evaluating the effect of PT, Figure 5.5 shows details for MWNT/PDMS, the only additive to reach PT for the wt\%'s evaluated. Figure 5.5(a) shows the resistance versus pre-strain relationship for MWNT/PDMS at $0.1,0.5$, and $1 \mathrm{wt} \%$ concentrations (note resistance axis is a $\log$ scale). At $0.1 \mathrm{wt} \%$, the composite is essentially an open circuit, however by $0.5 \mathrm{wt} \%$, a tell-tale drop of approximately three orders of magnitude in resistance occurs, which signals crossing PT. For composites greater than PT, as prestrain increases a corresponding increase in resistance is witnessed. Pre-straining the sample resulted in a decrease in the number of nanotube junctions that determine overall network resistance ${ }^{211}$. Figure 5.5(b) shows this effect as well as that of NIR illumination on a $1 \mathrm{wt} \%$ sample. Due to the resistance axis being a linear vice log scale as in Figure 5.5(a), increase in NIR off resistance with increasing pre-strain is easier to observe. Figure 5.5(b) also shows regardless of pre-strain, a decrease in resistance with NIR illumination, signaling electron-hole $(e-h)$ pair generation or electrostatic interactions. Photo-conductivity dependence of composites on pre-strain is intriguing and could be quite useful as adaptive skins for structural health monitoring of aerospace frames, where strains could be continuously monitored using photo-conductive measurements. The resistance value for a given carbon concentration and pre-strain can be modeled by 

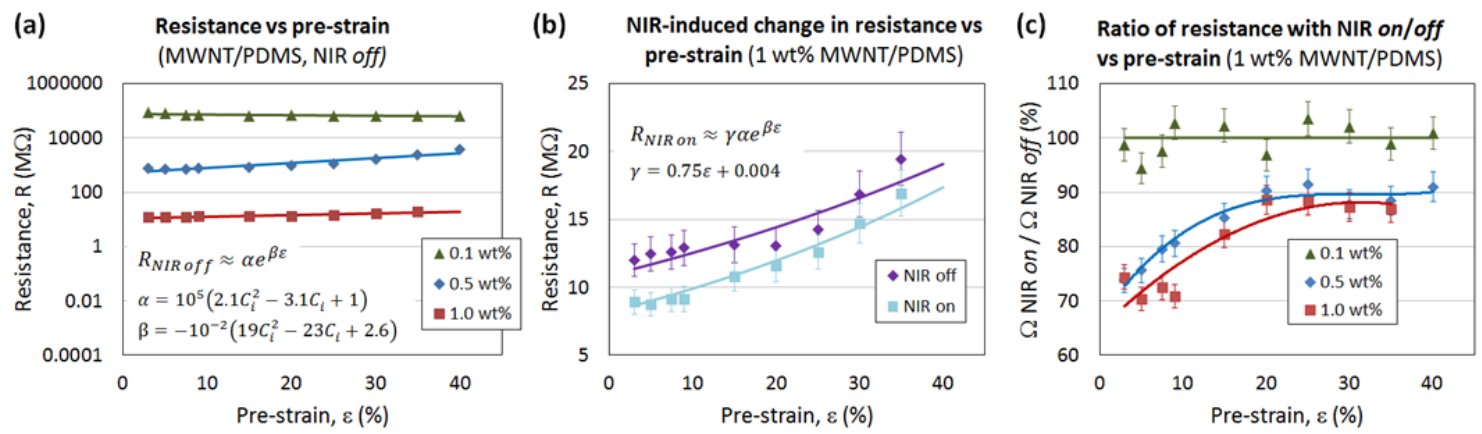

Figure 5.5. Photo-induced changes in conductive composites. (a) Comparison of NIR off resistance measurements for various MWNT concentrations, PT is crossed between 0.1 and $0.5 \mathrm{wt} \%$. (b) Detail showing increasing NIR off and on resistance curves as a function of pre-strain. (c) Ratios NIR on/off resistance showing increasing photo-conductivity effect with increasing carbon loading.

standard geometric power functions $R_{\mathrm{NIR} \text { off }}=\alpha \mathrm{e}^{\beta \varepsilon}$ and $R_{\mathrm{NIR} \text { on }}=\gamma \alpha \mathrm{e}^{\beta \varepsilon}$ with two concentration fitting parameters ( $\alpha$ and $\beta$ ), and an illumination factor $(\gamma)$, which is a function of pre-strain $(\varepsilon)$. As pre-strain increases, the effect of NIR illumination diminishes. At a pre-strain of 3\%, NIR on resistance decreases by $\sim 30 \%$; however, by a pre-strain of $40 \%$, the drop is just $10 \%$. Figure 5.5(c) more clearly illustrates this effect, which shows the ratio of NIR on to NIR off for various wt $\%$ concentrations. As it is less than PT, the $0.1 \mathrm{wt} \%$ curve shows no change in resistance as a result of NIR illumination. By $0.5 \mathrm{wt} \%$ however, carbon loading is above PT; and thus, the ratio of NIR on/off shows a measurable resistance change. For $0.5 \mathrm{wt} \%$, this change runs from $\sim 75 \%$ at $3 \%$ prestrain to $\sim 95 \%$ at $40 \%$ pre-strain. As wt $\%$ loading increases, the effect also increases, as is shown by the $1 \mathrm{wt} \%$ curve, which runs from $\sim 70 \%$ at $3 \%$ pre-strain to $\sim 90 \%$ at $40 \%$ pre-strain. These results suggest that establishment of a connective network of carbon 
additive in the polymer is a critical consideration for such extraordinary photo-mechanical response. Dependence of photo-conductivity on applied pre-strains of

the composites suggests $e-h$ pair generation due to interband transitions ${ }^{64,212}$. It is possible that adding small amounts of low dimension carbon nanostructures, such as CNTs, to higher dimensional carbons, such as SLG, GNP, or HOPG, will create mixeddimensional composites with artificially lowered PTs resulting in enhanced actuation and photo-conductivity.

\subsection{ACTUATION EFFICIENCY AS A FUNCTION OF DIMENSIONALITY}

Figure 5.6 provides an overview of the dimensional dependent photo-mechanical responses. Figure 5.6(a) shows change in stress as a function of concentration $(0.01-1 \mathrm{wt} \%)$ in the four carbon additives. As described previously, for a given concentration, the lowest dimension (MWNTs, 1D) has the highest response. As dimensionality increases, response decreases. Figure 5.6(b) shows the photo-mechanical stress response dependence of laser power for a $1 \mathrm{wt} \%$ concentration. This response also follows a dimensionality relationship, and was modeled by $\Delta \sigma \approx \alpha[1-\exp (-P / \beta)]$, with dimensionally dependent coefficients ( $\alpha$ and $\beta$ ) and laser power $(P)$. Corresponding curve fitting equations for both $\Delta \sigma$ and efficiency $(\eta)$ as function of carbon nanostructure concentration $\left(C_{\mathrm{i}}\right)$ and dimension $\left(d_{\mathrm{i}}\right)$, are given in Figures 5.6(a) and (c) respectively. The slope of the curves shows that lower dimension carbon additives are more efficient at converting light to photo-responses than their higher dimensional counterparts. Figure 5.6(c) shows in greater detail efficiency as a function of concentration (all for $40 \%$ pre-strains). Since photo-response was highest at $40 \%$ pre-strain, this value was chosen to display for efficiency values. Figure 5.6(d) details the efficiency for MWNT/PDMS 


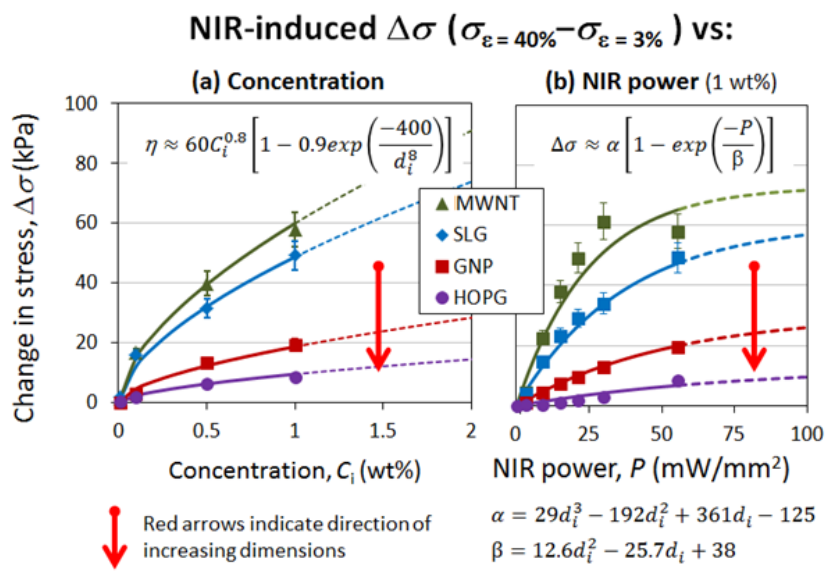

NIR-induced efficiency vs:
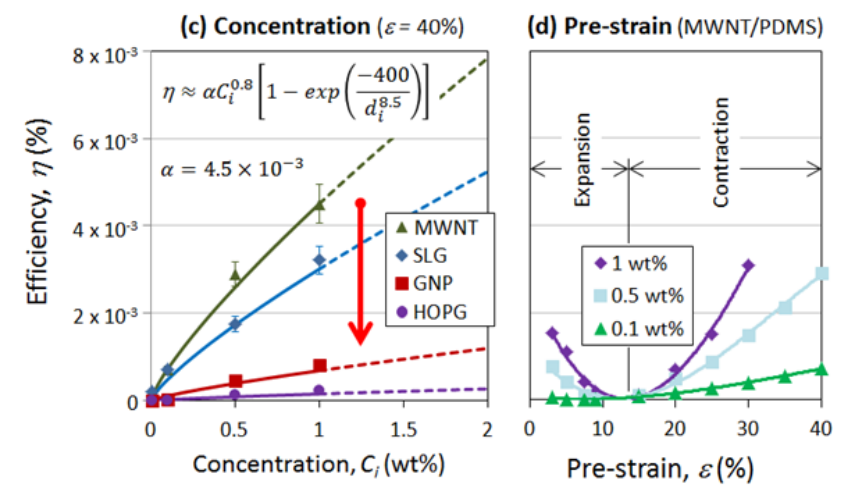

Figure 5.6. Dimensional dependence of photo-mechanical response. (a) NIR-induced change in stress (from a pre-strain of 3\% to $40 \%$ ) as a function of both concentration and laser power for a fixed $1 \mathrm{wt} \%$. (b) Efficiency vs. concentration and pre-strain.

sample at $0.1,0.5$, and $1 \mathrm{wt} \%$. Since efficiency shows effectiveness of converting laser power into a measurable response, the efficiency curves show similar trends as the relative stress change. At low pre-strains, there is a slight expansion of the composite; therefore, efficiency is small. At moderate pre-strains, composites have a negligible response, thus the efficiency is also negligible. Negligible efficiency merely indicates a balance between applied pre-strains and photo-mechanical forces. Finally at high pre-strains, samples showed a large contraction, thus a large associated efficiency. It is possible that efficiency is also greatly dependent on carbon loading with respect to PT. 
Crossing the PT leads to a more global response, thus activating more carbon and resulting in enhanced efficiency. Efficiency calculations are shown in Appendix C.

\subsection{CONCLUSIONS}

This chapter showcases the importance of dimensional state of carbon nanostructure in carbon/polymer composites for affecting overall photo-mechanical stress and efficiency responses. Carbon/PDMS composites were fabricated from four different carbon nanostructure dimensions, including: MWNTs (1D), SLG (2D), GNPs (2.5D), and HOPG (3D). An evaporative mixing technique was developed, which assisted in achieving homogeneity of difficult-to-disperse carbons within PDMS. Experimental results revealed several interesting dimensionally dependent and PT dependent characteristics. First, as dimensionality of carbon nanostructure additive in PDMS polymer decreases, NIR-induced photo-mechanical stress response increases. The lowest dimension additive (MWNTs, 1D) was the most effective at NIR photon absorption and subsequent energy transduction to the polymeric chains. This relationship held true both at various wt $\%$ loading of carbon nanostructures as well as NIR illumination intensities. A maximum observed stress change of $\sim 60 \mathrm{kPa}$ and $\sim 5 \times 10^{-3} \%$ efficiency were obtained with just $1 \mathrm{wt} \%$ MWNT loading, which is two orders of magnitude higher than for commercial polymers such as polyvinylidene fluoride ${ }^{193}$, and azobenzene containing polymers and gels ${ }^{194}$. Likewise, efficiency also follows the same dimensional dependency, with lower dimensional carbon nanostructures (such as MWNTs) more efficient at converting NIR light to photo-mechanical responses than their higher dimensional counterparts (such as HOPG). On the other hand, actuation and relaxation 
kinetic responses were found to be related not to dimensionality, but rather to PT of carbon nanostructure additive in the polymer.

Composites at wt \%'s less than PT responded with nearly identical actuation and relaxation times, regardless of dimensionality. However, composites at wt $\%$ 's greater than PT responded not only slower for both actuation and relaxation, but also had a higher magnitude of photo-mechanical stress response. This indicates that establishing a connective network of carbon nanostructure additive allowed for energy transduction responsible for the photo-mechanical effect to activate carbon beyond the NIR illumination point, changing from a localized (at the NIR illumination point) to a global (in the entire composite) response. The crossover from local to global response carries both benefits - such as greater overall photo-mechanical stress and higher efficiencies, as well as drawbacks - such as longer actuation and relaxation times required to reach steady-state conditions. However, these longer actuation and relaxation times are still measured on a timescale in seconds, as opposed to other photo-mechanical composites such as shape memory polymers that require $\sim 60$ minutes for relaxation ${ }^{169}$. For composite samples greater than PT, photo-conductivity responses to NIR illumination were also measured and found to be dependent on $\mathrm{wt} \%$, as well as applied pre-strain. Increasing the $\mathrm{wt} \%$ loading in the composite increased the number of overall carbon junctions, resulting in decreasing test sample resistance. When composite samples greater than PT were exposed to NIR illumination, resistance decreased, showing $e-h$ pair generation within the composite reaching electrodes at the ends of the sample. These measurements also show connectivity of MWNTs in the samples. As increasing amounts of pre-strains were 
applied to the test composites, the amount of NIR illumination-induced change diminished.

Dimensional dependence of mechanical responses, electrical conductivity, optical properties, and magnetic properties of carbon nanostructures will be fundamental in developing future micro and nanodevices. Furthermore, systems based on two or more carbon nanostructures either in thin films or polymeric materials constitute "mixed-dimensional systems". For mixed-dimension systems, thermal conductivity, electron transport, photo-conductivity, thermoelectric power, and optical absorption are expected to be different compared to their single carbon nanostructure counterparts, and be dependent on geometry, anisotropy, and orientation of the carbon additives. First, at intersecting dimensional state junctions, density of states differences between 1D and 2D system can lead to modulation of electrical conductivity. Second, photo-conductivity could depend on mixed-dimensional states, possibly impacting a variety of optoelectronic and solar devices. Third, the thermoelectric power of graphene has been reported to be $\sim 80 \mu \mathrm{V} / \mathrm{K}$ at room temperature ${ }^{213}$, and that of carbon nanotubes $\sim 40 \mu \mathrm{V} / \mathrm{K}^{214}$. Therefore, mixed-dimensional systems consisting of nanotubes and graphene may exhibit built-in electric fields due to the difference in thermoelectric power. Fourth, differences in carbon dimensional state could be used to create composites with different optical absorption characteristics, which could impact infrared sensors ${ }^{215}$ and allow for photo-mechanical actuators with built-in optical filters. Fifth, recent reports on mixed-dimensional carbon forms [SWNT (1D) mixed with GNP (2.5D)] as fillers in polymeric materials resulted in higher thermal conductivity than their individual 
counterparts as fillers ${ }^{216}$. Synergistic effects that are created by interaction of CNTs with GNPs was shown to reduce the interfacial resistance for thermal conduction ${ }^{216}$.

In future applications, a variety of devices can be envisioned that exploit both dimensional and percolation dependent photo-mechanical traits of carbon nanostructured composites. For example, as fundamental materials for thin-film smart adaptive skins, localized actuation responses can be achieved in carbon nanostructure composites by keeping carbon loading at less than PT and incorporating a NIR laser control system that projects desired stress response patterns on the composite surface. Such a composite in aerospace applications could help replace traditional flight control surfaces. On the other hand, wide area deformations or stress responses can be achieved with carbon loading greater than PT and a single NIR source illuminating the composite, thus initiating global photo-mechanical responses. One possible application of a global response composite is that of a central NIR source surrounded by a multitude of pre-strained carbon nanostructured strips. NIR illumination on would cause strips to contract in unison, mimicking the function of muscle tissue. Such muscle tissues could serve as building blocks of future photo-mechanical robots. However, regardless of the eventual applications, fundamental knowledge of dimensionally dependent photo-response in both single and mixed-carbon nanostructure composites will enable researchers to approach complex challenges from an entirely different design philosophy for electronics, photonics, sensing, actuation, and biomedical applications.

Having evaluated fundamental characteristics of optically-induced responses in carbon nanostructure-based composites, the data clearly indicated that photo-mechanical actuation properties can be fine-tuned using a variety of factors (carbon dimensional 
state, $\mathrm{wt} \%$ loading, illumination intensity, etc.). While these effects are both interesting and have potentially broad applications, the combination of additional hybrid materials in the carbon nanostructure composites could enable large-scale macroscopic stimuli responsive properties. The next chapter introduces one such system using a combination of CNTs and thermally expanding microspheres in an elastomeric matrix to generate very large dimensional changes. While CNT-based composites were used in the following experiments (due to their low percolation threshold in PDMS), similar systems and responses could be obtained with a variety of carbon nanostructure additives. 


\section{CHAPTER 6 \\ STIMULI-RESPONSIVE TRANSFORMATION IN CARBON NANOTUBE/EXPANDING MICROSPHERE-POLYMER COMPOSITES}

\subsection{INTRODUCTION}

By integrating thermally expanding microspheres (TEMs) into a CNT/elastomer composite, this chapter presents a sample stimuli-responsive system with ability to undergo large, macroscopic dimensional changes. A decade of nanotube/polymer composite research has yielded interesting insights in molecular design and mechanical properties of nanocomposites. Carbon nanotube fillers have been used to increase strength $^{217}$; as stress recovery agents in thermoplastic elastomers and IR actuators ${ }^{51,52,166}$; to improve damping capability ${ }^{218}$; as shape/temperature memory composites ${ }^{219}$; as space durable films for electrostatic charge mitigation ${ }^{220}$; to improve flammability resistance ${ }^{221}$; as conductive scaffolds for printable composites and gels ${ }^{222,223}$; as electro-responsive chromatic materials $^{224}$; and as skin-like pressure and strain sensors ${ }^{225}$. The combination of high strength, light weight, and large elastic energies in these composites have been proposed for diverse applications ranging from high-end sports equipment ${ }^{226}$ to artificial muscles in humanoid robots ${ }^{227}$. While important, many of these applications merely incorporate nanotubes to accentuate already existing host matrix features (such as strength, toughness, conductivity), there has been little success in development of 
stimuli-responsive composites that exhibit dynamic changes in strength, conductivity, density, and volume (visible on macroscopic scales).

A stimuli-responsive ternary composite with the ability to unidirectionally transform from an initial elastic state to a more rigid plastic-like state upon thermal or IR stimuli is reported. Addition of TEMs and SWNTs in a PDMS elastomer matrix resulted in a material with ability to on-demand undergo volume expansion ( $>500 \%)$, density reduction ( $>80 \%$ ), elastic modulus increase $(>675 \%)$, and resistance changes. Volume expansion is achieved through dispersion of core-shell microspheres in the composites. Consisting of a gas-tight acrylic copolymer shell encapsulating liquid hydrocarbon, TEMs have two states -initial (unexpanded) and expanded [Figure 6.1(a) shows to-scale cutaway]. Heating the copolymer shell above its glass transition temperature softens the shell and vaporizes the encapsulated hydrocarbon. The subsequent internal pressure rise plastically deforms the shell, resulting in permanent volume increase ${ }^{228}$ [Figures 6.1(b) and (c)]. While TEMs are commercially available in a range of expansion temperatures, ones selected for use were at the lower end of the spectrum in order to facilitate use of low intensity IR illumination to induce remote expansion $\left(T_{\text {start }} \sim 105^{\circ} \mathrm{C}\right.$ and $T_{\max }$ $\sim 140^{\circ} \mathrm{C}$ ). Using scanning electron microscopy, initial microsphere diameter was determined to be $7.1 \pm 1.9 \mu \mathrm{m}$, and expanded $17.8 \pm 3.8 \mu \mathrm{m}$ (yielding $150 \%$ diameter, $500 \%$ surface area, and 1,500\% volume increases). Dispersion of SWNTs and TEMs in PDMS was accomplished using an evaporative mixing $\operatorname{method}^{53}$, and resulted in 


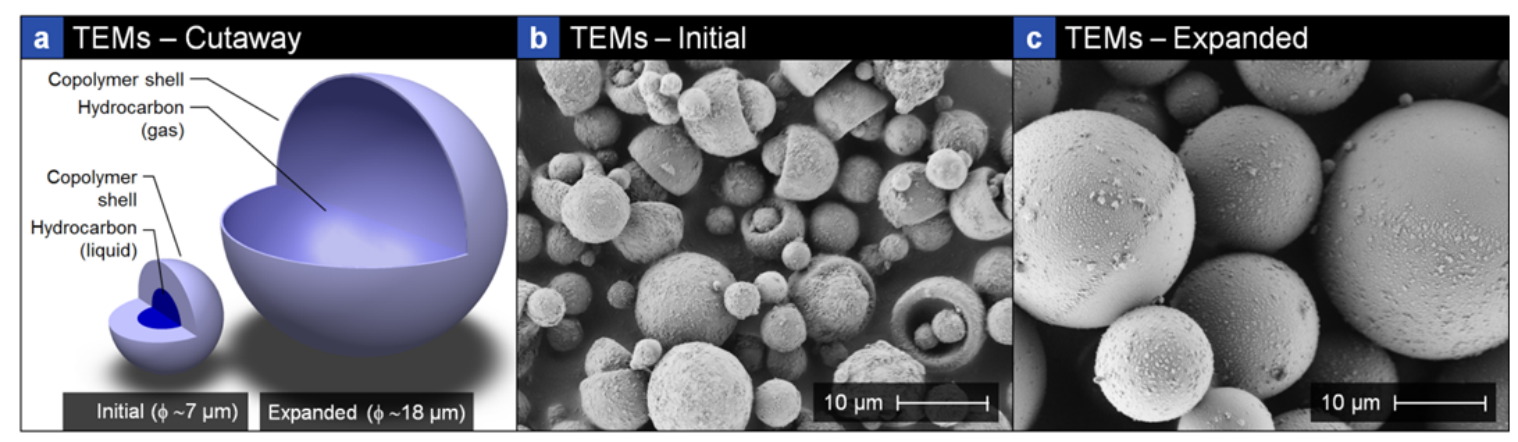

Figure 6.1. Expanding microsphere overview. (a) To-scale cutaway showing size relationship between initial and expanded microspheres. (b) SEM of loose unexpanded microspheres (average diameter of $7.1 \pm 1.9 \mu \mathrm{m}$ ). (c) SEM of loose expanded microspheres (average diameter of $17.8 \pm 3.8 \mu \mathrm{m}$ ).

interesting stimuli-responsive mechanical and electrical property transformations. Figure 6.2 shows the relationship between SWNT loading and composite resistivity $(\rho)$. The $\rho$-wt $\%$ curves associated with each dispersion method will give a measure of relative dispersion method efficiency. Curves shifted up or to the right represent less efficient dispersion methods, while curves shifted to the left or down are more efficient. Appendix D details composite fabrication steps.

\subsection{STIMULI RESPONSIVE TRANSFORMATION}

Incorporation and subsequent thermal expansion of TEMs in SWNT/polymer composites results in interesting changes to the polymer matrix. Upon application of thermal or IR stimuli, microsphere expansion results in an eye-observable transformation of the initial (as-fabricated) elastic polymer composite into a plastic-like state.

Figure 6.3(a) shows the smooth surface of an initial fabricated ( $0.3 w t \%$ SWNT, $31.2 w t \%$ TEM) unexpanded composite. In this image, neither SWNTs nor TEMs are 


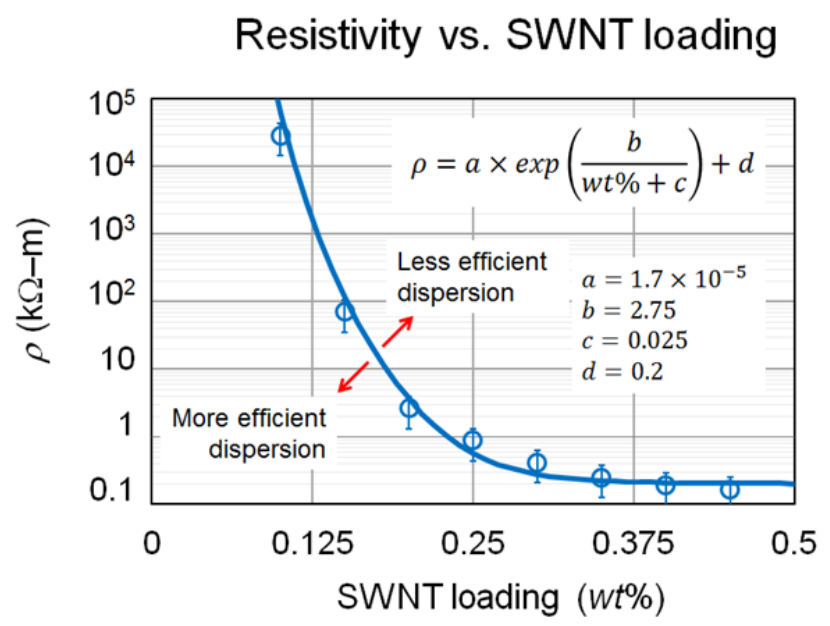

Figure 6.2. Plot of resistivity $(\rho)$ versus SWNT $w t \%$ loading (data fit to a NIST MGH10 nonlinear regression equation).

discernible from the bulk polymer matrix; however, post expansion [Figure 6.3(b)] individual microspheres are clearly seen bulging out of the bulk polymer. Examination of the area between adjacent microspheres shows the matrix has been stretched into local thin $(<1 \mu \mathrm{m})$ polymer films, the thickness of which was observed to be inversely proportional to microsphere loading.

In composite materials, observations have demonstrated that at the filler-polymer interface - chain mobility is restricted (confirmed via nuclear magnetic resonance measurements ${ }^{229}$ ) resulting in a thin region of glassy-like behavior ${ }^{230-233}$. Chain mobility restriction decreases as distance from the filler increases, following a gradient back to the bulk matrix ${ }^{232}$. In initial composites, glassy regions surrounding unexpanded microspheres are relatively small compared to the bulk polymer [as Figure 6.3(c) shows]. Since the majority of bulk polymeric chain mobility remains unaffected, macroscopic composites retain elastic properties. As TEMs expand, however, the matrix between adjacent microspheres stretches into local submicron polymer films, emulating 
substantially greater filler loading. Due to TEM surface area increasing on expansion, corresponding polymer chain restrictions also increase [Figure 6.3(d)], resulting in macroscopic transformation of the entire system from an elastic to plastic-like state. Resulting polymer mobility between adjacent filler particles is hindered, possibly forming glassy-like flexible bridges ${ }^{232}$. Such expansion-induced alteration of the polymeric chain conformations observed here could be useful in adaptive and responsive interface development.

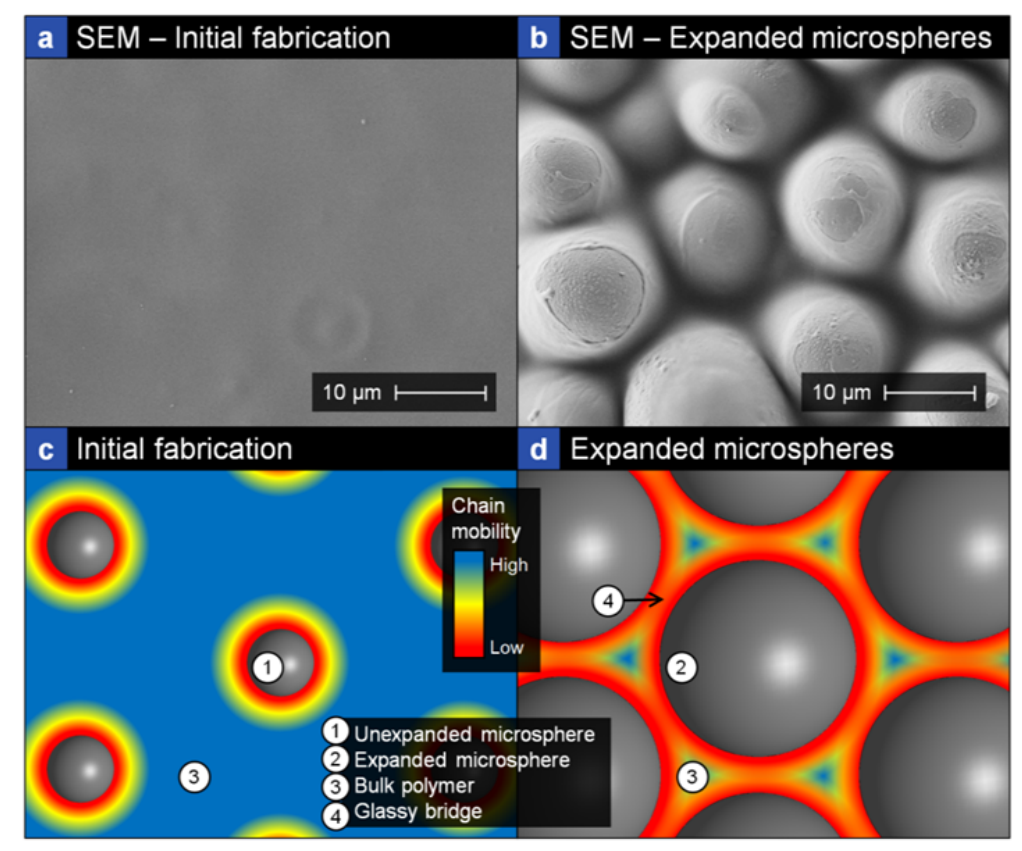

Figure 6.3. Effects of microsphere expansion. (a) SEM image showing smooth surface of the initial fabricated composites [0.3 wt\% SWNT, $31.2 w t \%$ TEM]. (b) SEM of the same composite sample after expansion, with microspheres clearly visible. (c) Unexpanded TEMs exhibit little influence over bulk composite properties. (d) The larger surface area associated with expanded TEMs, however, greatly inhibits polymer chain movement and results in macroscopic material property changes. 


\subsubsection{BINARY MECHANICAL PROPERTIES}

Because reinforcing materials are uniformly distributed and randomly oriented in the PDMS matrix, fabricated composites exhibited isometric dimensional and volumetric expansion. Figure 6.4(a) shows percent change of these quantities as a function of increasing TEM $w t \%$. Theoretical as well as $\pm 1 \sigma$ volume expansion curves (based on average TEM unexpanded/expanded size analysis) are also included. Since composite mass is constant but volume increases upon TEM expansion, there is a corresponding decrease in density. Figure 6.4(b) compares initial and expanded density as a function of TEM loading, with theoretical curves included here as well. Because theoretical and $\pm 1 \sigma$ curves were determined based on measurements of loose TEMs not in a matrix, slight underperformance of experimental results compared to theoretical values is expected. While volumetric expansions up to $>900 \%$ were witnessed (45 wt $\%$ TEM), subsequent experimental work was done with $31.2 w t \%$ samples. This loading still yields an impressive volume increase of $>500 \%$ and density reduction of $>80 \%$ while maintaining composite integrity. Figure 6.4(c) is an expanded composite cross-section showing morphology detail of several hollow microspheres and polymer-coated SWNTs.

While high TEM $w t \%$ is desirable from an expansion perspective, there are associated drawbacks. Increased microsphere loading lowers the PDMS matrix to reinforcing material ratio. Since the PDMS matrix provides polymeric chains responsible for binding the composite, filler loading can be large enough such that resulting structures are not stable and tend to break apart or crack upon expansion. This response was observed to occur above $\sim 50 w t \%$ TEM loading. While volumetric expansions up to $>900 \%$ were witnessed (45 wt\% TEM), subsequent experimental work was done with 
$31.2 w t \%$ samples. This level yields an impressive volume increase of $>500 \%$ and density reduction of $>80 \%$ while maintaining composite integrity. A side-by-side comparison of initial and expanded composites at various TEM loadings is shown in Figures 6.5(a) and (b).
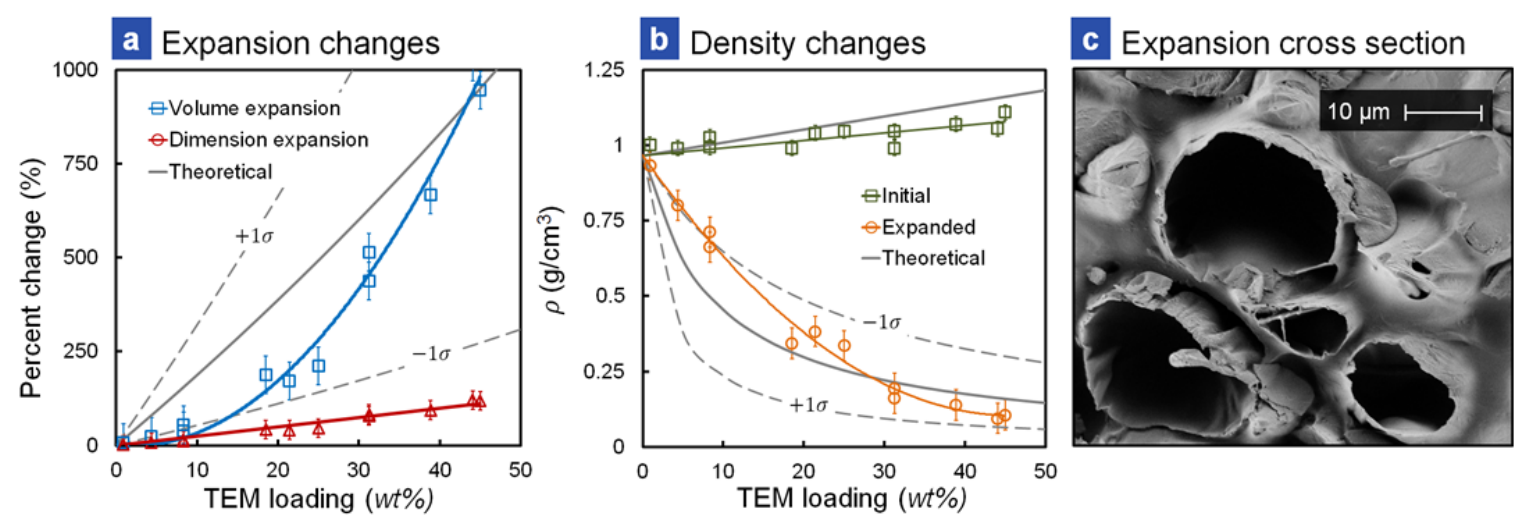

Figure 6.4. Expansion and density overview. (a) Expansion versus TEM $w t \%$ plot including dimensional and volumetric curves. (b) Density versus TEM $w t \%$ plot showing reduction between the initial and expanded composites. (c) SEM cross-sectional image of the expanded composite, showing large cavities inside the microspheres as well as several polymer-coated SWNTs.

While SWNT $w t \%$ was used as the standard throughout this work (which remains constant regardless of TEM state), it is interesting to note the decrease in volume fraction $\left(V_{f}\right)$ of SWNTs due to microsphere expansion. Equation (6.1) was used to transform $w t \%$ to Vol\%. Variables $\rho_{S W N T}$ and $\rho_{\text {TEM/PDMS }}$ are the densities of the SWNTs and TEM/PDMS matrix, respectively. A SWNT density of $2.1 \mathrm{~g} / \mathrm{cm}^{3}$ (per manufacturer's documentation), and TEM/PDMS densities of $1.045 \mathrm{~g} / \mathrm{cm}^{3}$ and $0.178 \mathrm{~g} / \mathrm{cm}^{3}$ for initial and expanded samples, respectively (based on experimental data) were used. Figure 6.5(c) shows the 

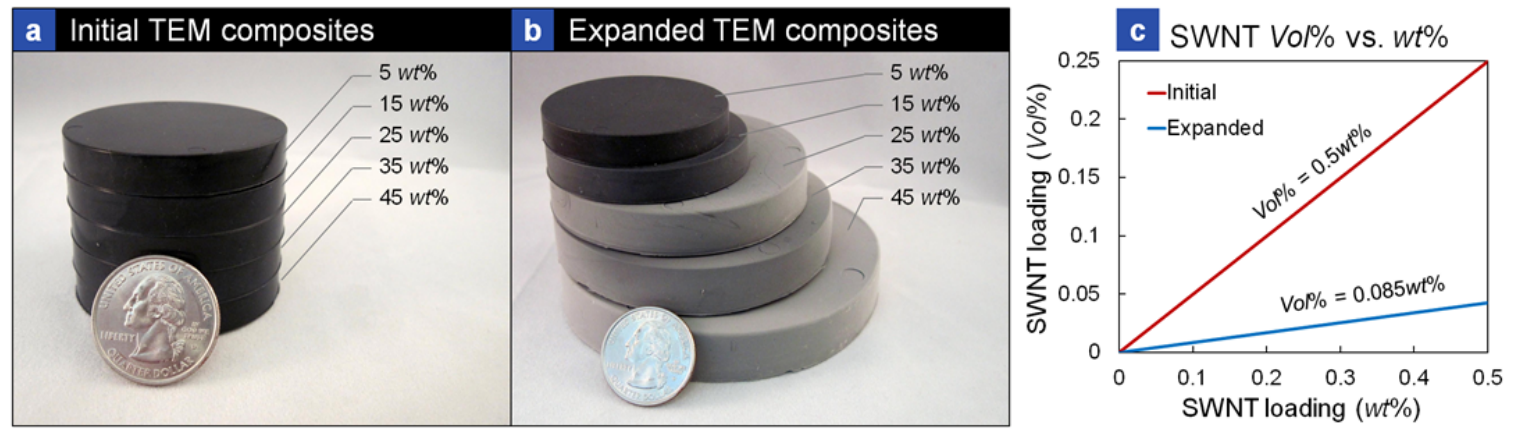

Figure 6.5. Volume and dimensional expansion plugs. Standard size plugs $(45 \mathrm{~mm}$ diameter, $8 \mathrm{~mm}$ height) were used to determine expansion changes as a function of increasing TEM $w t \%$. (a) Initial (as-fabricated) expansion plugs, in the initial or elastic state. TEM loading levels from $5 w t \%$ (top plug) to $45 w t \%$ (bottom plug) were evaluated. (b) Expanded, or plastic-like, plugs. A relationship between increased TEM loading and resulting volumetric increase is clearly visible. Also interesting to note, as TEM $w t \%$ increases, color change in the expanded composites is more noticeable compared to the initial plug. While $w t \%$ of all constituents remains constant, TEM expansion alters effective volumetric percentage. (c) Relationship between volume percent and $w t \%$ for SWNT loading. Expanded (upper) curve illustrates volumetric increase (and thus density reduction) resulting from microsphere expansion.

relationship between $w t \%$ and $V o l \%$. For a given SWNT loading, microsphere expansion results in no change in $w t \%$, but $\sim 80 \%$ reduction in $V o l \%$.

$$
V o l \%=\frac{\frac{w t \%_{S W N T}}{\rho_{S W N T}}}{\frac{w t \%_{S W N T}}{\rho_{S W N T}}+\frac{100-w t \%_{S W N T}}{\rho_{T E M / P D M S}}}
$$

While volumetric and density changes are the most visual effects, they are merely a façade for interesting synergistic effects between SWNTs, TEMs, and the polymer 
matrix. Just as microsphere expansion causes macroscopic dimensional changes, expansion also alters loading distribution within the composites. To examine these effects, engineering stress $\left(\sigma_{e}\right)$ versus engineering strain $\left(\varepsilon_{e}\right)$ for an initial and expanded $0.3 w t \%$ SWNT, $31.2 w t \%$ TEM sample were evaluated [Figure 6.6(a)]. Because expansion induced volume increase (and correspondingly cross-sectional area increase) was due to void generation in the TEMs, the initial (unexpanded) area provides a reasonable value to use for both initial and expanded stress calculations. Interestingly, not only did the composites exhibit elastic stress responses in the initial (unexpanded) state, but they also followed Hooke's law $\left(\sigma_{e} \approx E \varepsilon_{e}\right)$ up to $\sim 25 \%$ strain. After microsphere expansion however (upper curve), significant variations in composite strength existed and Hooke's law applied in a much smaller region, reaching the proportional limit at $\sim 1 \%$ strain. Expansion of a $0.3 w t \%$ SWNT, $31.2 w t \%$ TEM composite resulted in a remarkable $675 \%$ increase in Young's modulus $(E)$ as compared to the same $w t \%$ unexpanded sample (4.7 MPa initial, $37 \mathrm{MPa}$ expanded), similar to other nanotube/polymer composite systems ${ }^{234}$. Furthermore, microsphere expansion also transformed the classic elastic stress-strain responses seen in the unexpanded composites into something more indicative of a plastic material. Hence, these expanded composites are referred to as "plastic-like".

After initial fabrication, carbon nanotubes were randomly oriented and untensioned, resulting in minimal effect on elastic properties. However, microsphereinduced expansion and subsequent thin film formation in composites was observed to result in nanotube tensioning. Figures 6.6(b) and (c) are SEM images of $0.3 w t \%$ SWNT, $31.2 w t \%$ TEM samples at $40 \%$ strains. Crease lines imparted from the polymer onto the 


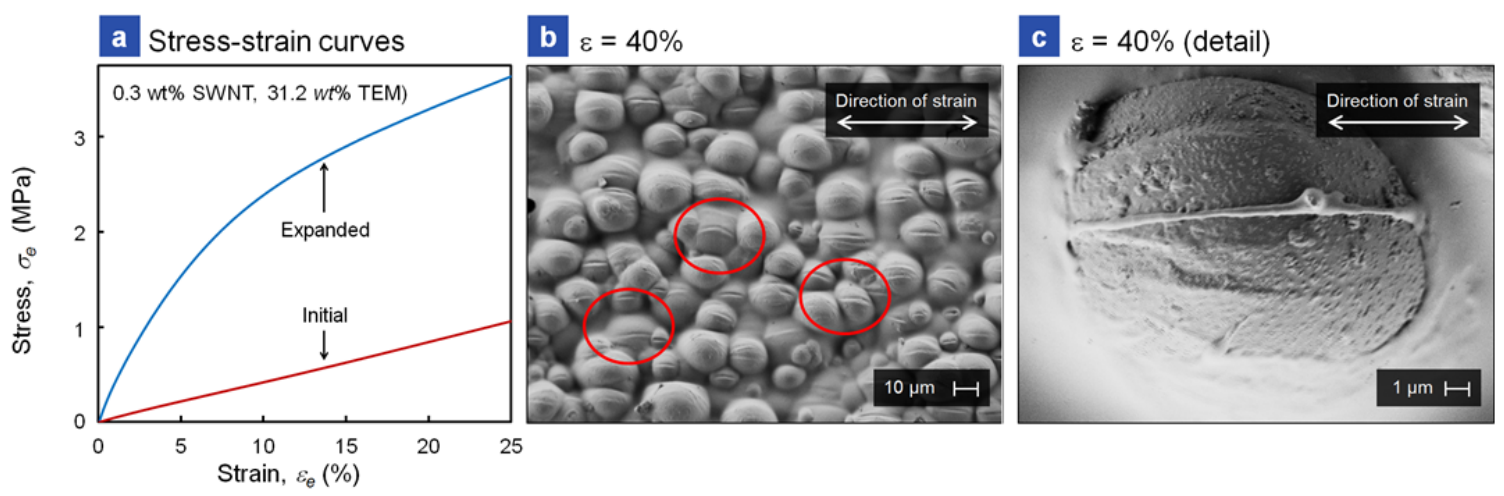

Figure 6.6. Stress-strain overview. (a) Composite stress-strain curve ( $0.3 w t \%$ SWNT, $31.2 w t \%$ TEM). Lower curve is initial (elastic) response, upper curve is expanded (plastic-like) response. (b) SEM image of expanded composite ( $0.3 w t \%$ SWNT, 31.2 $w t \% \mathrm{TEM})$ at $40 \%$ strain. Circled areas highlight several polymer-induced creases on the microspheres. (c) SEM image of expanded composite (0.3 $w t \%$ SWNT, $31.2 w t \%$ TEM) showing polymer-covered SWNT stretched across expanded TEM at strain of $40 \%$. Nanotube is bridging bulk polymer on either side of the microsphere.

TEMs are clearly visible in Figure 6.6(b), with several circled for clarity. Figure 6.6(c) shows a polymer-covered SWNT stretched across an expanded microsphere acting as a nanotether and providing strength to the expanded composite. Additional examples are shown in Figures 6.7(a) and (b), which are SEM images clearly showing carbon nanotubes acting as tethers in an expanded composite. While still randomly oriented, it is believed that nanotube tensioning will result in enhanced load transfer. Furthermore, due to random orientation, the strengthened bulk composites will retain beneficial isotropic properties, vice anisotropic properties typically associated with aligned carbon nanotube composites $^{235}$. These results are supported by past reports that show composites with aligned as opposed to random nanotube distributions are three times more effective as 
reinforcement materials ${ }^{236}$. Expansion-induced tensioning of carbon nanotubes has not been reported in previous studies and could represent a paradigm in stimuli-responsive composites.

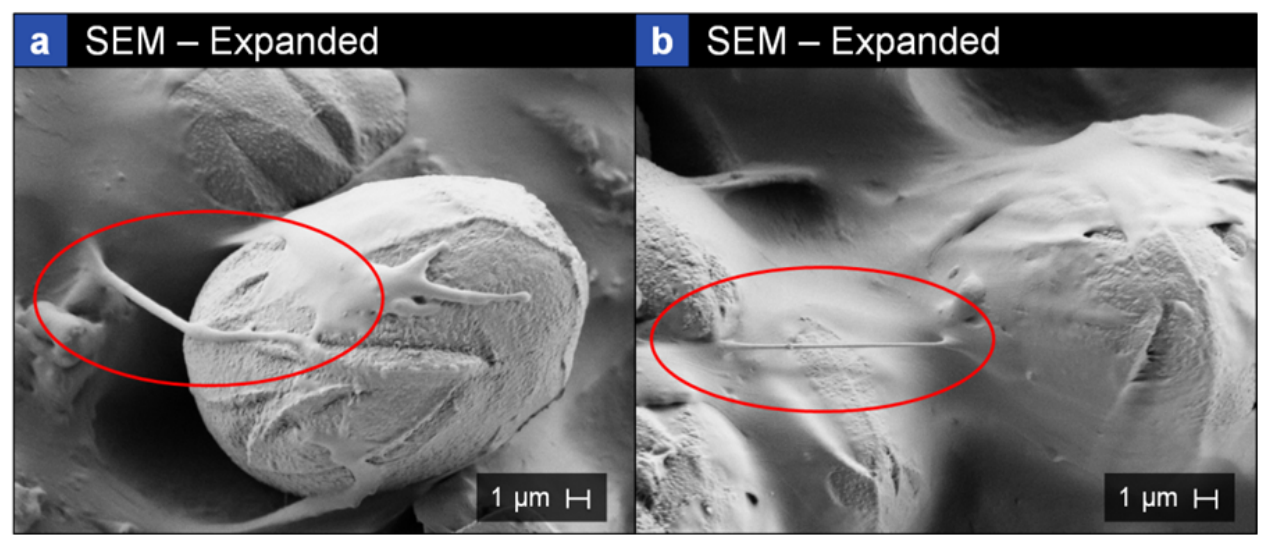

Figure 6.7. SWNTs imparting mechanical strength in expanded composites. (a), (b) Both SEM images show cross-sectional views of expanded composites $(0.3 w t \%$ SWNT, $31.2 w t \%$ TEM). Polymer-coated SWNTs can be seen bridging, or acting as a nanotether, between expanded TEMs and bulk polymer matrix. Microsphere-induced expansion tensions nanotubes. This results in enhanced load transfer in expanded composites, forming the basis for increases in mechanical strength.

\subsubsection{ELECTRICAL CHANGES DUE TO SHIFTING NANOTUBE JUNCTIONS}

Effects of microsphere expansion are not limited solely to transformations in mechanical properties. Because of SWNTs' 1D-morphology, microsphere expansioninduced rearrangement of nanotube junctions causes corresponding electrical property shifts. IR-induced TEM expansion was used to alter amount of overlap, or electrical junctions, between adjacent SWNTs. Composite samples were exposed to $45 \mathrm{~s}$ of IR point source illumination [850 nm light emitting diodes (LEDs)] while continuously monitoring resistance and expansion rate. The ratio of post-expansion $\left(R_{f}\right)$ resistance to 
initial $\left(R_{0}\right)$ resistance $\left(R_{f} / R_{0}\right)$ as a function of SWNT loading (represented by resistivity, $\rho$ ) was utilized to evaluate electrical relationships. Note that low $\rho$ results from high SWNT $w t \%$, and vice versa. In all experiments, TEM loading was kept at $31.2 w t \%$, while SWNT ratios were varied. Intriguingly, $R_{f} / R_{0}$ exhibited four different $w t \%$ dependent electrical responses, including becoming more conductive, less conductive, transitioning from conductive to insulating, or remaining insulating.

Figure 6.8(a) is a plot showing $R_{f} / R_{0}$ versus initial sample resistivity. Near the plot center, a percolation threshold region is shown as the vertical pink bar; resistivities lower than this are conductive, and higher are insulating. At high $w t \%$ (low $\rho$ ), SWNT loading is much greater than the percolation threshold. Here (region 1), TEM expansion and subsequent nanotube junction rearrangement results in composites becoming more conductive $\left(R_{f} / R_{0}<1\right)$. As SWNT loading lowers and $\rho$ increases (region 2), amount of conductive overlap between adjacent nanotubes decreases as the microspheres expand. Even after expansion, however, there is still enough nanotube contact to retain conductivity; therefore, region 2 loading results in samples becoming slightly more resistive and therefore less conductive $\left(1<R_{f} / R_{0}<10\right)$. As SWNT loading is further lowered to just greater than the percolation threshold (region 3), microsphere expansion again stretches junctions between adjacent nanotubes. SWNT loading is low enough, however, that as microspheres expand, electrical contact between nanotubes is either broken or greatly reduced. This reduction in contact area results in substantial resistance increase $\left(R_{f} / R_{0}>10\right)$. Expansion of these composites can essentially be thought of as an off switch. Finally for insulating composites where SWNT loading is less than 

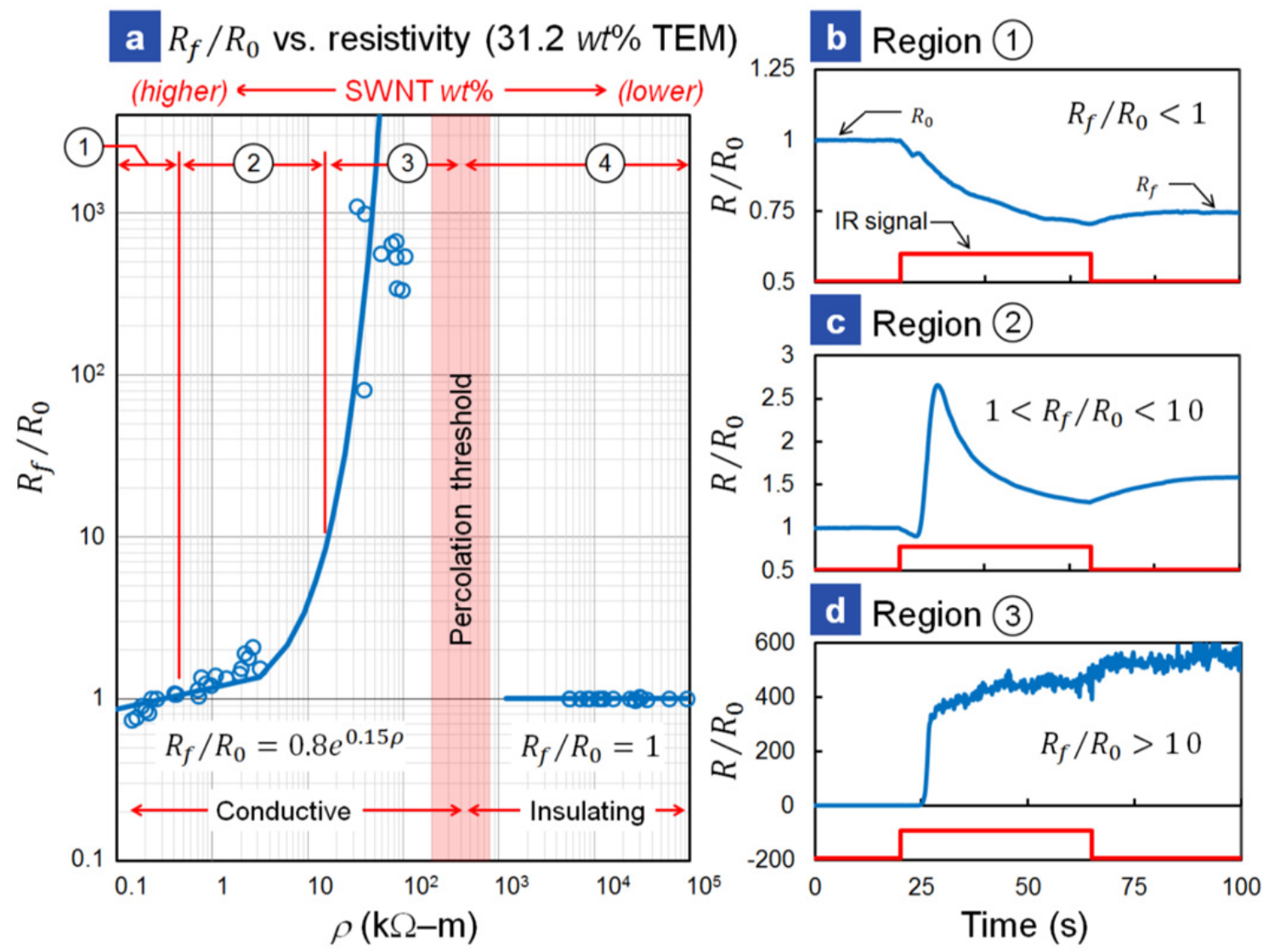

Figure 6.8. Electrical overview and response regions 1-3 (Region 4 not shown as insulating nature results in no change). (a) Plot of change in sample resistance $\left(R_{f} / R_{0}\right)$ versus initial resistivity. Response regions (1-4) as well as percolation threshold are indicated on the graph. (b)-(d), Detail resistance changes of the first three regions versus time.

percolation threshold (region 4), there was no observed change in electrical properties. Conductive sample data was fit to the exponential function $R_{f} / R_{0}=0.8 e^{0.15 \rho}$ while insulating data fit to $R_{f} / R_{0}=1$.

In addition to tunable $R_{f} / R_{0}$ resistance relationships, distinct time-dependent (and IR illumination-induced) resistivity patterns were observed. Figures 6.8(b)-(d) show 
change in resistance (plotted as $R / R_{0}$ ) versus time for the first three regions. Region 4 is not shown as there are no $R / R_{0}$ changes. Red lines indicate IR illumination signals. Prior to IR illumination on, resistance level is steady in regions $1-3\left(R / R_{0}=1\right)$. At $20 \mathrm{~s}$, IR LEDs are energized. Immediately for all three regions, IR-induced heating begins and thermally generated electrons and optically generated electron-hole pairs begin to occur in the conductive samples, resulting in resistance decrease (dip in $\left.R / R_{0}\right)$. As the composites quickly reach microsphere expansion temperature $(\sim 3 \mathrm{~s})$, composite dimensions start to increase in the area of IR illumination. By $\sim 5 \mathrm{~s}$ after illumination, composite expansion rate is significant enough to dictate response. For region 1, high SWNT loading, junction stretching plays a minor effect and only a slight bump up is seen. For region 2, SWNT loading is lower, and junction stretching causes an increase in $R / R_{0}$ as microsphere expansion reduces SWNT conductive overlap. As expansion reaches $\sim 80 \%, e-h$ pair again dominates and $R / R_{0}$ trend reverses. In region 3 , TEM expansion causes a significant (or "open circuit" depending on $w t \%$ ) reduction of nanotube junctions. After IR illumination is turned off $(65 \mathrm{~s})$ all regions show an increase in $R / R_{0}$ and resistance increases to an overall higher steady state value.

Two additional interesting effects were noticed. First, for the same IR illumination time and intensity, samples with higher SWNT $w t \%$ exhibited larger regions of microsphere expansion. This expansion indicates that associated increase in composite thermal conductivity resulted in a larger portion of the sample expanding in response to IR point source heating. More concisely, as electrical and thus thermal conductivity 
increase, microsphere expansion transitions from local to global effect. Second, the presence of nanotubes was required for a composite to exhibit IR-induced expansion as well as photon-induced resistance change. Regardless of illumination time, plain $31.2 w t \%$ TEM samples ( $0 w t \%$ SWNT) exhibited negligible expansion - indicating nanotubes were efficient at photon absorption and subsequent energy transduction, and required for IR-induced expansion. The increasing relative expansion area with increased nanotube loading in Figures 6.9(a) and (b) show SWNTs are efficient at IR absorption and subsequent thermal transduction to polymeric chains. As nanotube loading increases, increased thermal conductivity in the composites results in a larger expansion region (starts transitioning from local effect to global one). IR illumination $(850 \mathrm{~nm})$ is initiated

a IR point source expansion vs. time

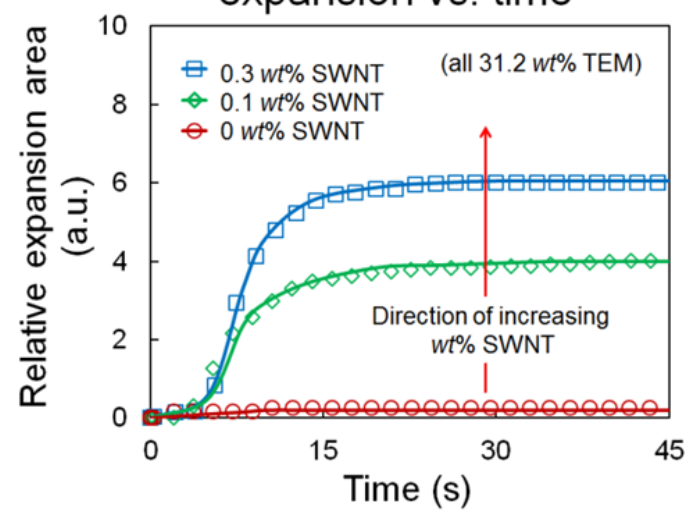

b IR-expanded composite test samples

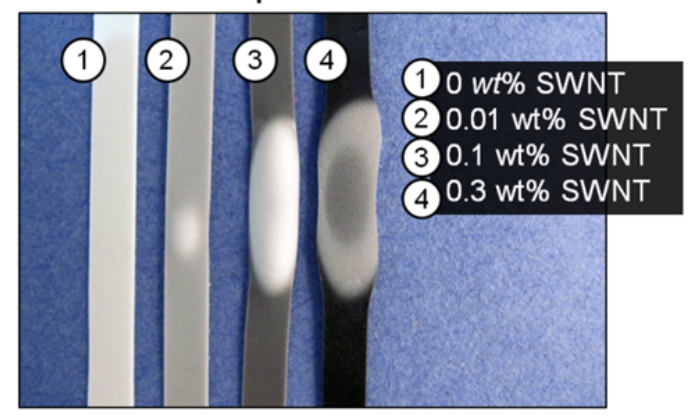

Figure 6.9. Relative IR-induced expansion in various $w t \%$ SWNT composites. (a) Amount of IR-induced expansion in $0,0.1$, and $0.3 w t \%$ SWNT composite samples (all $31.2 w t \%$ TEM). (b) Image showing IR-expanded composite test samples. SWNT $w t \%$ increases from left (no nanotubes) to right ( $0.3 w t \%$ nanotubes). All strips shown are $31.2 w t \%$ TEM and were subjected to identical IR test conditions. 
at time $\mathrm{t}=0 \mathrm{~s}$. Inclusion of even a small amount of SWNTs allows for some expansion, as is shown when comparing the $0.01 w t \%$ (minor expansion area) to the $0 w t \%$ (no expansion). It is remarkable that nanotubes with diameters almost three orders of magnitude smaller than the microspheres result in IR-induced TEM expansion.

\subsection{ELECTRICAL APPLICATIONS}

Since $R_{f} / R_{0}$ is controllable via SWNT $w t \%$, these results can be used for creating embedded thermally actuated polymer circuits or switches with dynamically controllable electrical properties. Expansion start and stop positions can be controlled via IR modulation or selection of TEMs with either higher or lower expansion temperatures. A TEM/SWNT/PDMS circuit can be embedded within a larger plain PDMS structure, heating and subsequent expansion will only occur within the patterned circuit (IR absorption by the SWNTs ${ }^{237}$ ), while the bulk PDMS (optically clear ${ }^{190}$ ) remains cool. Figure 6.10 shows once such circuit and a corresponding fabrication sequence. Using results obtained from Figure 6.2 to tune resistors' electrical responses, this circuit can be

designed such that depending on which resistor element(s) are IR illuminated, the circuit can become more conductive, less conductive, or maintain the same conductance (Appendix E contains IR response calculations for this circuit design). The fabrication sequence is as follows: [1] lower encapsulation layer is deposited; [2] SWNT/PDMS with nanotube loading greater than the percolation threshold is deposited to form conductive traces (note equivalent circuit diagram shown in this step); [3] two separate regions of different $w t \% \mathrm{SWNT} / \mathrm{TEM} / \mathrm{PDMS}$ are patterned forming resistors $R_{1}$ and $R_{2}$; [4] a plain PDMS filler layer is used to equalize the substrate and circuit feature height; and [5] upper encapsulation layer which seals the circuit. 
a Fabrication sequence

(1)

Lower encapsulation layer

$\square$ Plain PDMS

(optically clear)

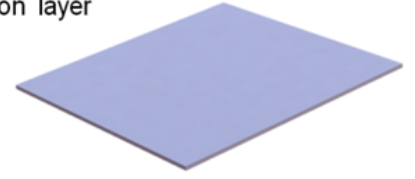

(2) Conducting trace layer

SWNT/PDMS

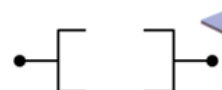

(3)

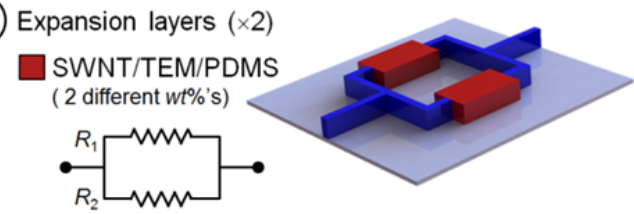

(4) Filler layer

$\square$ Plain PDMS (optically clear)

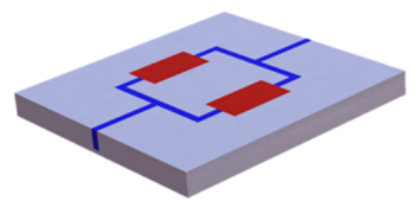

(5) Upper encapsulation layer

$\square$ Plain PDMS (optically clear) b Final circuit

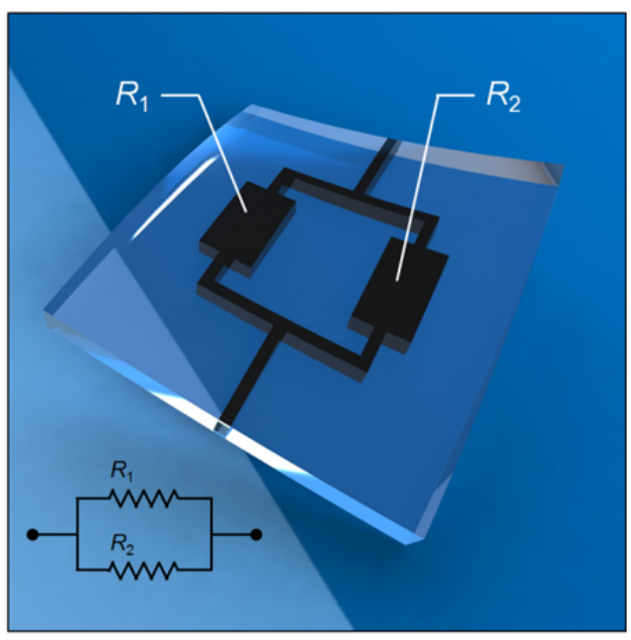

Figure 6.10. Embedded circuit fabrication sequence. Sequence presented shows steps required to embed a simple parallel resistor circuit into a flexible polymer skin. (a) Fabrication sequence, note that arbitrary colors are used to help illustrate different layers in the process. (b) Rendering of the SWNT/TEM circuit inside of its transparent plain PDMS shell. IR illumination of the structure will cause heating only in the polymer region containing SWNTs (and subsequent microsphere expansion in resistors $R_{1}$ and $R_{2}$ ).

\subsection{CONCLUSIONS}

Even though carbon nanostructure/TEM composites only allow for one-time expansion/actuation, the innovative combination of filler materials in an elastomer matrix 
and resulting binary set of material properties presents a plethora of research and industrial applications. Possible applications encompass fields such as structural health monitoring of aircraft ${ }^{238}$ and ships ${ }^{144}$, deformable elements for flight control surfaces ${ }^{239}$, deformable mirror and antennae surfaces on spacecraft ${ }^{240}$, strain sensing for roads and bridges $^{241}$, machinery vibration control systems ${ }^{242}$, and biomedical applications including micropumps ${ }^{243}$ and targeted drug delivery ${ }^{244}$. Ternary combination of SWNTs, TEMs, and PDMS allows for design of independently controllable regions of variable mechanical strength, electrical conductivity, and expansion in the same polymer composite. For example, thin-patterned polymer films can be incorporated into vehicle and/or equipment skins, and expanded to provide on-demand structural elements to prevent system failure [Figure 6.11(a)]. In biomedical applications, carbon nanostructure/TEM composites provide a possible new stent material [Figure 6.11(b)]. In actuation applications, even though the TEMs cause an overall system expansion, contractive forces can still be obtained [Figure 6.11(c)]. For example, this contracting skin requires four layers to fabricate [Figure 6.12(a)]; the steps involved include: [1] patterning a lower expansion layer comprised of SWNT/TEM/PDMS (these areas will expand with IR illumination); [2] placement of a lower filler layer of SWNT/PDMS (no TEMs) in the regions between expanding strips (filler sections will not expand upon IR illumination); [3] patterning as upper expansion layer to align on top of the lower filler layer (the two stacked expansion layers act as a bimetallic strip, inducing a curling force in the skin upon IR illumination); and [4] deposition of an upper filler layer in order to duplicate curling in the opposite direction and obtain a contractive expanding response. 


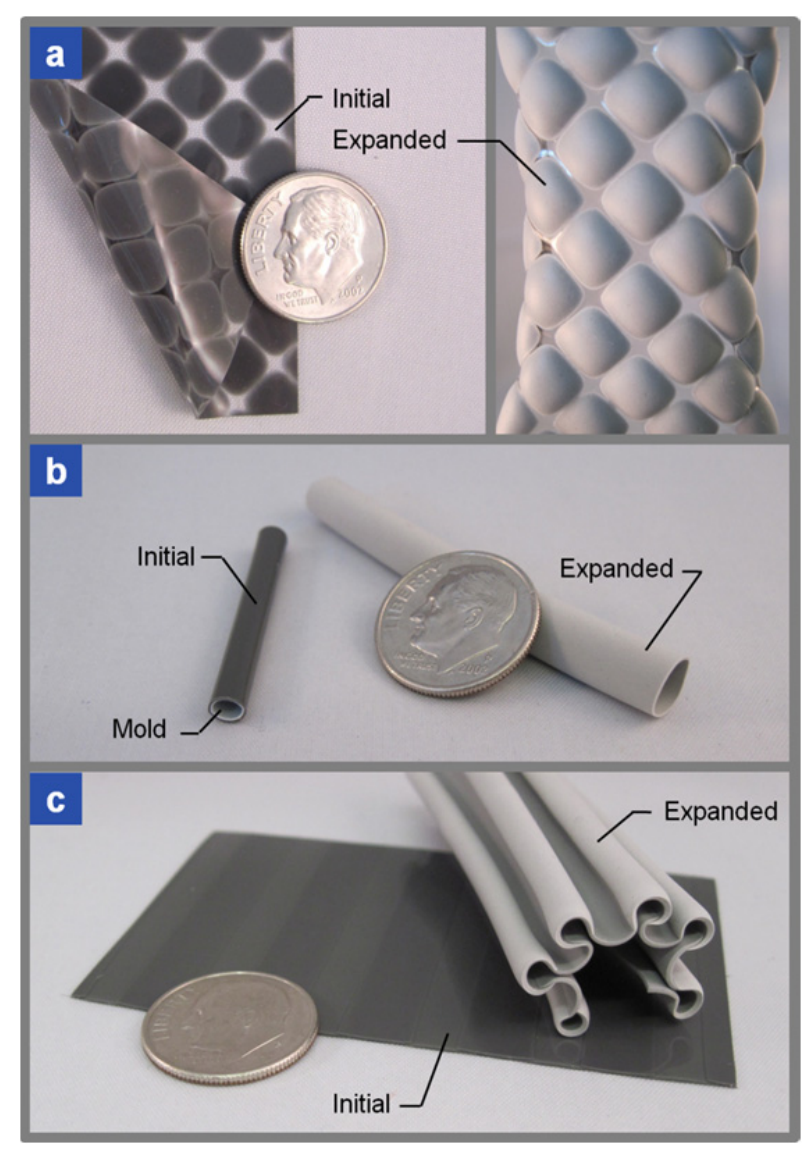

Figure 6.11. Assorted applications. Ternary combination of SWNTs, TEMs, and PDMS allows for design of independently controllable regions of variable mechanical strength, electrical conductivity, and expansion in the same polymer composite. (a) Patterned polymer films showing possible structural element. (b) Unexpanded composite on a mold is shown at the left of the figure (future molds can incorporate IR LED elements). These stents can be inserted into region of interest, and LEDs energized to induce expansion. (c) Thin polymer film patterned to curl up on itself, thus generating a contractive force. 
a Fabrication sequence

(1) Lower expansion layer SWNT/TEM/PDMS

(2) Lower filler layer SWNT/PDMS

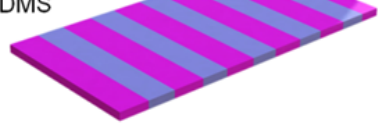

(3) Upper expansion layer SWNT/TEM/PDMS

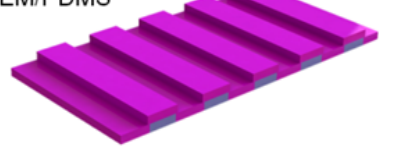

(4) Upper filler layer SWNT/PDMS b Expansion detail

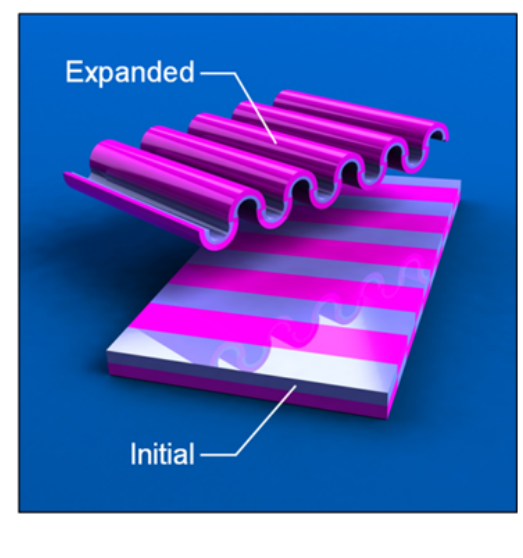

Figure 6.12. Contracting skin fabrication sequence. (a) Steps [1] to [4] detail the four layers required to achieve a contractive skin response. (b) Rendering showing resulting initial and expanded composite skins.

Elastic to plastic-like transformation allows for incorporation of sensing and actuation into a single coherent functional material thereby minimizing complexity and maximizing reliability. Furthermore, benefits of IR-induced microsphere expansion include wireless actuation, electro-mechanical decoupling (and therefore low noise), and massive parallel actuation of device arrays from a single light source. Focused IR illumination can be used to expand a specific area or the entire composite. Ability to pattern various filler compositions into a single polymer skin allows for a diverse set of properties (such as regions of variable expansion, conductivity, and strength) in one continuous composite, and then change these properties on-demand for structural and/or electrical components. For example, thin unexpanded flexible elastic polymer films can 
be incorporated into vehicle and/or equipment skins, and later expanded to provide ondemand structural elements preventing system failure. In biomedical applications, carbon nanostructure/TEM composites provide a possible stent material. Although TEMs cause overall system expansion, contractive forces can be obtained through selective microsphere patterning [Figures 6.11(c) and 6.12]. In applications where volume is critical, such as aerospace and space travel, thin elastic polymer films can be fabricated [Figure 6.13(a)] and subsequently rolled-up for shipping or launch. Once on station, these films can be expanded [Figure 6.13(b)] to function as electrical, mechanical, and structural components. Finally, such composites could be highly useful in lab-on-a-chip applications, flexible bioelectronics, and sensors. Flexible skins could include microfluidic channels that expand volume on demand to create reaction chambers for live analysis of captured rare cells, such as circulating tumor cells.

This work introduces a class of stimuli-responsive expanding polymer composites with ability to unidirectionally transform physical dimensions, elastic modulus, density, and electrical resistance. Carbon nanotubes and core-shell acrylic microspheres were dispersed in polydimethylsiloxane, resulting in composites that exhibit a binary set of material properties. Upon thermal or infrared stimuli, liquid cores encapsulated within the microspheres vaporize, expanding the surrounding shells and stretching the matrix. Microsphere expansion results in visible dimensional changes, regions of reduced polymeric chain mobility, nanotube tensioning, and overall elastic to plastic-like transformation of the composite. Here composite transformations including macroscopic volume expansion $(>500 \%)$, density reduction $(>80 \%)$, and elastic modulus increase ( $>675 \%)$ are shown. Additionally, conductive nanotubes allow for remote expansion 


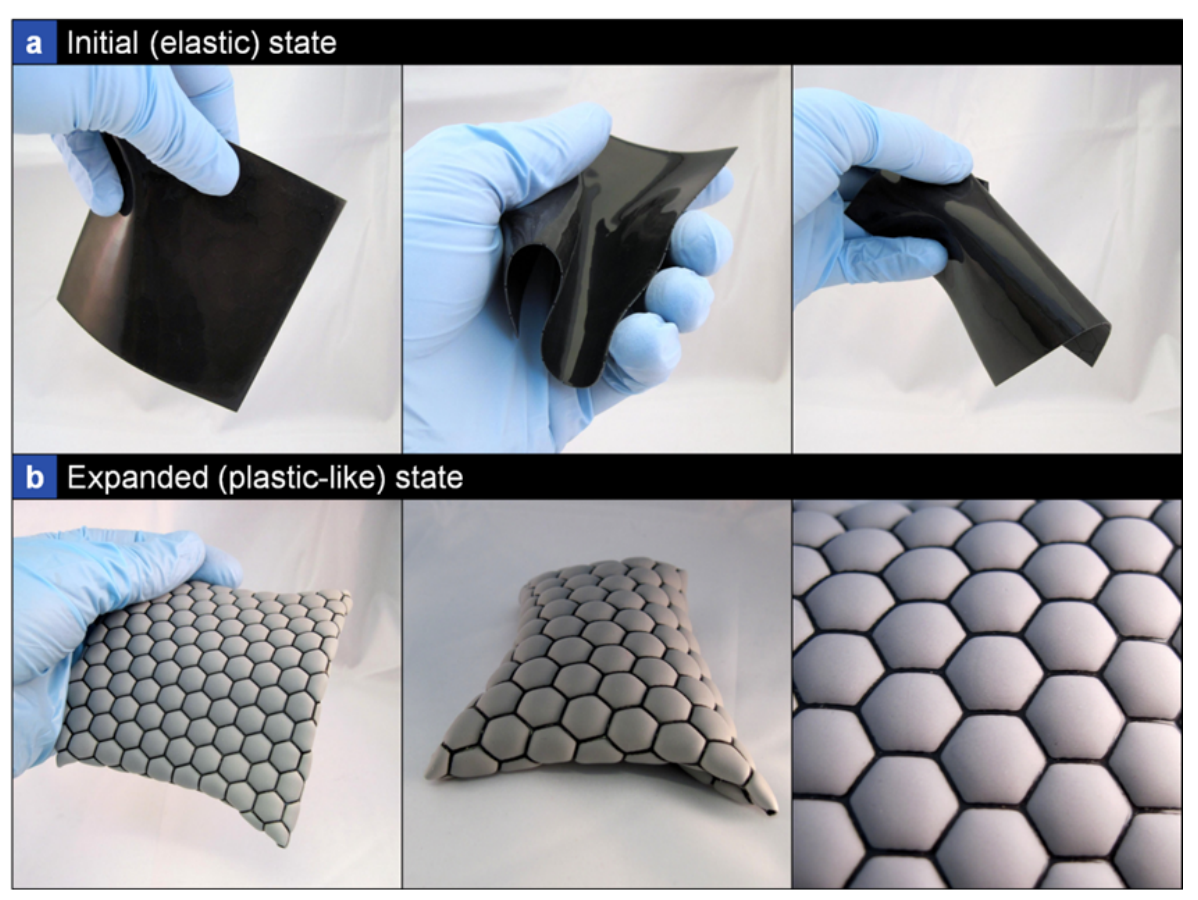

Figure 6.13. Application example showing use of patterned TEM regions to serve as structural component. (a) As-fabricated polymer sheet shown in elastic (unexpanded) state. (b) Same polymer sheet, but with expanded (plastic-like) honeycomb-patterned TEM regions.

monitoring and exhibit distinct loading-dependent electrical responses. With ability to pattern regions of tailorable expansion, strength, and electrical resistance into a single polymer skin, these composites present opportunities as structural and electrical building blocks in smart systems. 


\section{CHAPTER 7 \\ GRAPHENE/ELASTOMER COMPOSITE-BASED PHOTO-THERMAL NANOPOSITIONERS}

\subsection{INTRODUCTION}

As opposed to the unidirectional macroscopic actuation just discussed, this chapter describes development and characterization of a reversible photo-mechanical nanopositioning application demonstrated with a two-axis translation stage. Compared to piezoelectric actuators, which serve as the current workhorse for nanopositioning/manipulation, a closed-loop two-axis light-driven (photo-thermal) nanopositioning system capable of controlled displacements of more than $100 \mu \mathrm{m}$ per axis, actuation speeds exceeding $5 \mu \mathrm{m} / \mathrm{s}$, sub-micron resolution, and low-cost was developed. The unique photo-thermal positioning mechanism was achieved via dynamic modulation of multiple independent infrared sources placed along the actuators that enabled differential control through polymeric chain stretching/contraction. Disposable composite actuators were fabricated using graphene nanoplatelet loadings from $0-2 \mathrm{wt} \%$ and thicknesses from 90-210 $\mu \mathrm{m}$, and exhibited long-term stability ( $>3,000$ cycles $)$ and opto-mechanical efficiency of $\sim 0.03 \%$. This nanopositioning system based on a simple graphene/elastomer model system demonstrates a real-world application for low-cost energy-efficient positioning that could find utility in future photo- and electro-thermal actuation technologies. 


\subsection{RESULTS}

\subsubsection{PRINCIPLE OF GNP/ELASTOMER PHOTO-THERMAL ACTUATION}

Figure 7.1(a) presents a schematic demonstrating the principle GNP/elastomer photo-thermal actuation. Unrestricted elastomeric composites with graphene additives (sample [1]) have random polymeric chain arrangement/entanglement. Addition of weight (sample [2]) to the free-end stretches the composites and induces a pre-strain. As entangled polymeric chains are pulled into a more ordered arrangement, system entropy is reduced. When the pre-strained composite sample is heated via IR illumination (sample [3]), light is absorbed by the GNPs within the PDMS matrix; and optical energy is

efficiently transduced into thermal energy through phonons in the $\mathrm{sp}^{2}$ graphene sheets ${ }^{245}$. Due to the high macroscopic thermal conductivity of graphene films ${ }^{246}(300 \mathrm{~W} / \mathrm{m} \cdot \mathrm{K})$ and intimate dispersion of GNPs within the PDMS matrix, heat is percolated through the matrix causing polymeric chain contraction. Increasing polymeric chain temperature results in an associated increase in spring constant ${ }^{247}$. Since there is constant force applied (suspended weight), contraction in the composite occurs (following Hooke's law) and lifts the weight, resulting in usable work done through the system by so called "rubbery elasticity". The large amplitudes of actuation obtainable in graphene/elastomer and nanotube/elastomer systems are directly as a result of the $\mathrm{sp}^{2}$ bonding in the nanocarbons. In general, strong covalent $\mathrm{sp}^{2}$ bonds result in efficient heat transfer by lattice vibrations in the GNPs, and therefore allow for large amplitudes of photo-thermal actuation accomplished through heat transduction from the lattice into the polymeric chains. This thereby enables a unique photo-thermal actuation mechanism in nanocarbon/elastomer composites. In nanotubes and pristine single layer graphene, all 
bonds are strong $\mathrm{sp}^{2}$ covalent bonds that result in high intrinsic thermal conductivity ${ }^{66,248}$, while reduced graphene oxide is a mixture of $\mathrm{sp}^{2}$ and $\mathrm{sp}^{3}$ bonds. Finally, amorphous carbon and diamond-like carbon contain a large fraction of $\mathrm{sp}^{3}$ bonds that lower system thermal conductivity ${ }^{245}$. All of these forms can be useful for application-specific tunable photo-thermal actuation amplitudes in carbon/elastomer composites.

\subsubsection{PHOTO-THERMAL NANOPOSITIONING SYSTEM}

Figure 7.1(b) presents a simplified model of a single-axis photo-thermal nanopositioning system. In this setup, a thin GNP/PDMS composite strip is held at a fixed pre-strain, a nanopositioning stage is mounted in the composite center, and IR LEDs placed on either side for differential control via both positive and negative stage actuation (only one LED per side is shown for clarity, real system encompasses three LEDs per side, or six per axis). Illumination of the left LED results in localized heating of the GNP/PDMS composite around the light spot, causing polymeric chain contraction in that region and subsequent stage translation to the left. Conversely, energizing the LED on the right side will cause the stage to translate right. By dynamically modulating IR intensity, a force balance can be enacted on either side of the stage to maintain it in a desired displacement. However, even when the stage is at a set position, LED intensity is still being continuously tuned via a proportional-integral-derivative (PID) control loop which monitors stage position and compensates for thermal drift. This highly dynamic process achieves nanopositioning by controlling polymeric chain extension/contraction through rapidly modulating thermal energy input. Stage displacement is measured with the aid of high speed laser displacement sensor (120 nm resolution) and a reflective element placed on the stage. 


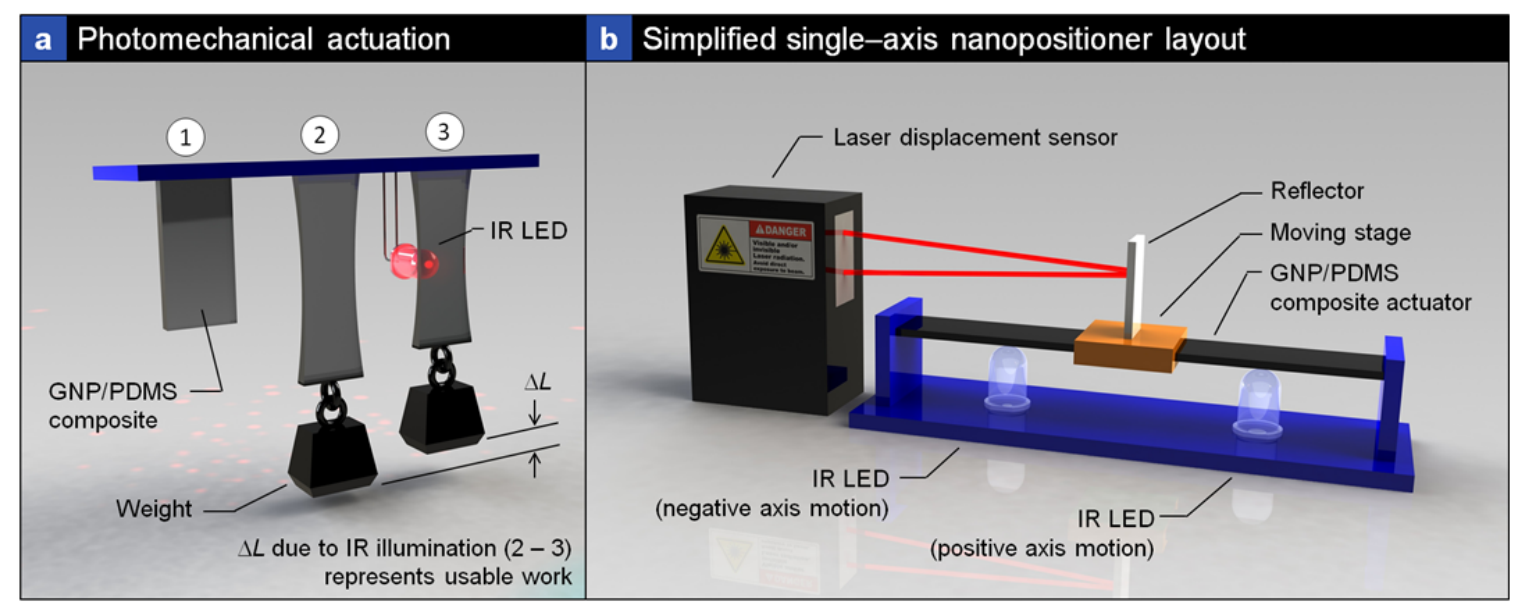

Figure 7.1. Photo-thermal overview. (a) Photo-thermal actuation schematic. Three thin GNP/PDMS composite strips are mounted with their upper ends fixed to a rigid plate. The free end of sample [1] is unrestricted, while samples [2] and [3] have a weight attached, thus inducing pre-strain into the composite actuator. [3] Illumination via an IR LED results in energy transduction to the polymeric chains, causing a contraction in the actuator (and thus usable work). (b) Simplified single-axis nanopositioner layout. A laser displacement sensor is used to measure stage position. Independently controlled diodes on either side of the stage allow for differential positive or negative axis stage motion.

\subsubsection{GNP/PDMS ACTUATOR FABRICATION AND CHARACTERIZATION}

Figure 7.2(a) presents a scanning electron microscope (SEM) image of GNPs deposited on a silicon wafer. GNPs were verified to be $3-5$ layers, with diameters ranging from 1-2 $\mu \mathrm{m}$. Figure 7.2(b) presents the photo-electron spectroscopy (XPS) of GNPs. The XPS C1s spectra shows the different binding energy values for different carbon bonds, namely sp ${ }^{3} \mathrm{C}-\mathrm{C}$ or $\mathrm{C}-\mathrm{H}(285.5 \mathrm{eV})$, single C-O bonds $(286.1 \mathrm{eV})$, and $\mathrm{sp}^{2} \mathrm{C}=\mathrm{C}$ bonds $(284.5 \mathrm{eV})$. Figure 7.2(b) shows the high levels of deoxygenated $\mathrm{sp}^{2}$ carbon bonds that exist in GNPs, signaling high intrinsic thermal conduction well-suited for 
photo-thermal actuators presented here. The intensity of $\mathrm{sp}^{2} \mathrm{C}=\mathrm{C}$ is much higher than the $\mathrm{sp}^{3} \mathrm{C}-\mathrm{C}$ bonds and C-O bonds. GNPs are still slightly oxidized on the surface as one can see the oxidized C-O bonds.

Investigating effects of varying fabrication parameters, Figure 7.2(c) is a series of five slides showing increasing composite opacity with a corresponding increase in GNP loading (from 0 to $2 \mathrm{wt} \%$ ). In all five slides, spin times and thus composite thickness are constant. Conversely, Figure 7.2(d) presents a series of four slides, all with identical GNP loading $(0.5 \mathrm{wt} \%)$, but decreasing spin times. See Appendix A for sample preparation steps. The longest spin time sample (90 s) is the thinnest and the most transparent, while the shortest spin time (15 s) is the thickest and most opaque. Compared to carbon counterparts such as single layer graphene (derived from reduced graphene oxide) and CNTs, GNPs are easy to disperse uniformly and thus are ideal for use in advanced polymer composites. The high level of $\mathrm{sp}^{2}$ carbon bonds, ease of dispersion in polymers via shear mixing, ability to spin-coat, and compatibility with MEMS lithographic processes makes GNP/PDMS more favorable for low-cost commercial manufacturing as compared to all other graphene/polymer composites. Quantitatively, Figure 7.2(e) shows curves of GNP/PDMS composite thicknesses as a function of GNP wt $\%$ loading for $15 \mathrm{~s}$ and 90 s spin times. GNP wt $\%$ was not observed to have a significant impact on thickness, rather it was observed to be spin time and spin speed-dependent, as shown in Figure 7.2(f). Finally, Figure 7.2(g) presents a GNP/PDMS composite actuator prior to mounting in the nanopositioner enclosure (a coin is shown for size comparison). The inset in this figure is a SEM image showing the composite smooth surface and several nanoplatelet flakes protruding from the cut edges. Fabrication of the polymer composites 
consisted of shear mixing GNPs in PDMS, spin-coating large area thin films, polymerization, and cutting $50 \mathrm{~mm} \times 6 \mathrm{~mm}$ actuator strips (strained $50 \%$ to $75 \mathrm{~mm}$ for use in nanopositioners).
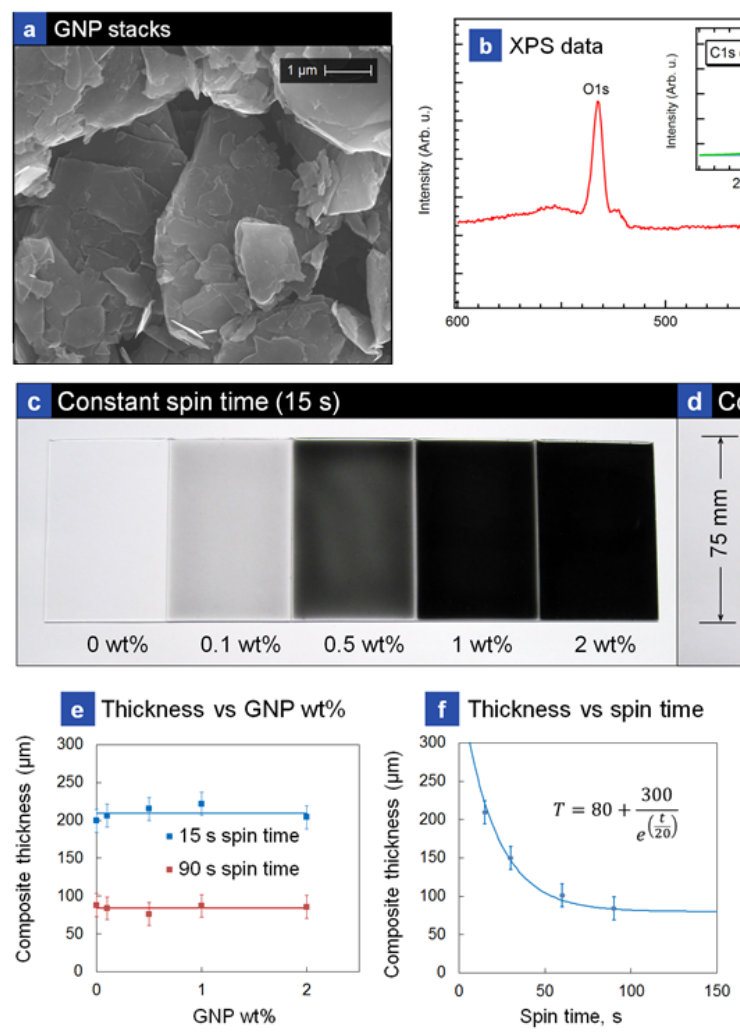

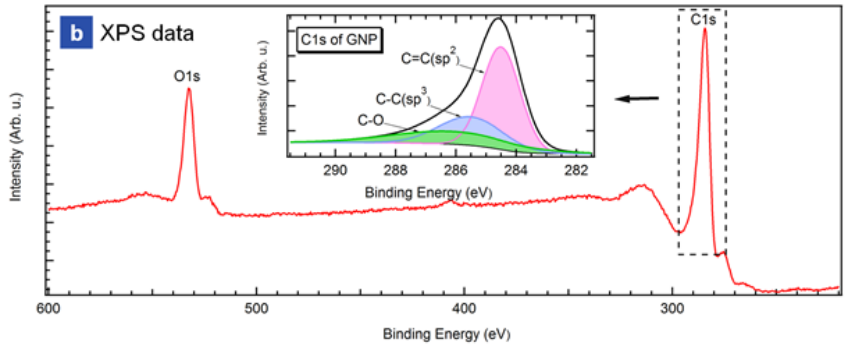

d Constant GNP concentration (0.5 wt \%)
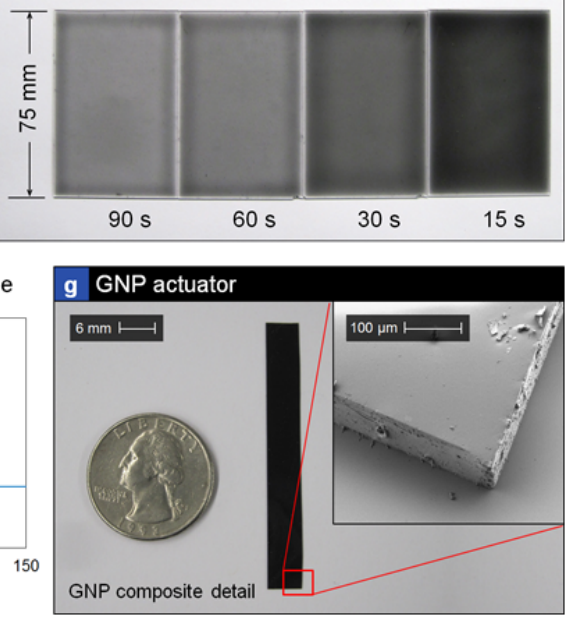

Figure 7.2. GNP/PDMS composite characterization. (a) SEM of plain GNP powder (not mixed with PDMS). (b) XPS data. (c) Changes in composite opacity as GNP loading is increased from 0 to $2 \mathrm{wt} \%$. In all five slides, spin time is constant at $15 \mathrm{~s}$. (d) Series of four slides with identical GNP loading $(0.5 \mathrm{wt} \%)$, but decreasing spin times. (e) GNP/PDMS composite thicknesses are shown as a function of GNP wt $\%$ loading. Curves for a $15 \mathrm{~s}$ spin time as well as $90 \mathrm{~s}$ spin time are displayed. (f) Composite thickness as a function of spin time is shown. Composite samples were fabricated with spin casting times between 15 s to 90 s. (g) Sample GNP composite actuator ( $2 \mathrm{wt} \%)$ used in our twoaxis nanopositioners. Inset is SEM detail showing a corner of the composite strip. 


\subsubsection{NANOPOSITIONER SYSTEM INTEGRATTION}

Following fabrication and characterization of graphene/elastomer composite actuators, they are integrated into a compact nanopositioning system incorporating a laser-cut enclosure housing multiple IR LEDs, custom electronics, and control software. Figure 7.3(a) presents an isometric overview of a fully assembled two-axis nanopositioner $(82 \mathrm{~mm} \times 82 \mathrm{~mm} \times 30 \mathrm{~mm})$. A removable cover plate encloses two GNP/PDMS actuators and their respective positioning diodes. A cutout in the cover plate allows for mounting a floating stage on the actuators underneath. Indicating lights on the front ( $x$ axis) and the left side ( $y$ axis) provide a visual operator tool by displaying positioning diode status. A single blue LED is illuminated on each axis indicating the nanopositioner is powered, since no other lights are lit the floating stage is in the home position $(0,0)$.

In Figure 7.3(b), the cover plate has been removed, exposing two GNP/PDMS composite actuators and their associated IR positioning LEDs. Six positioning diodes are mounted underneath each strained GNP/PDMS composite actuator strip (12 total for the two-axis system). The diode arrangement allows for independent differential stage control along both the $x$ and $y$ axes. While positioning diodes and GNP actuators are mounted to the top of the printed circuit board (PCB), all other electronics are mounted on the bottom side. Figure 7.3(c) presents a clarified top view of the nanopositioner with the cover plate and GNP actuators removed to show the diode layout. This orientation shows differential arrangement between positive and negative positioning diodes. Along their respective axes, IR LEDs closest to the home position [origin of $(0,0)$ ] were designated as " $\mathrm{C}$ ", those in the middle as "B", and the diodes farthest away as "A". 

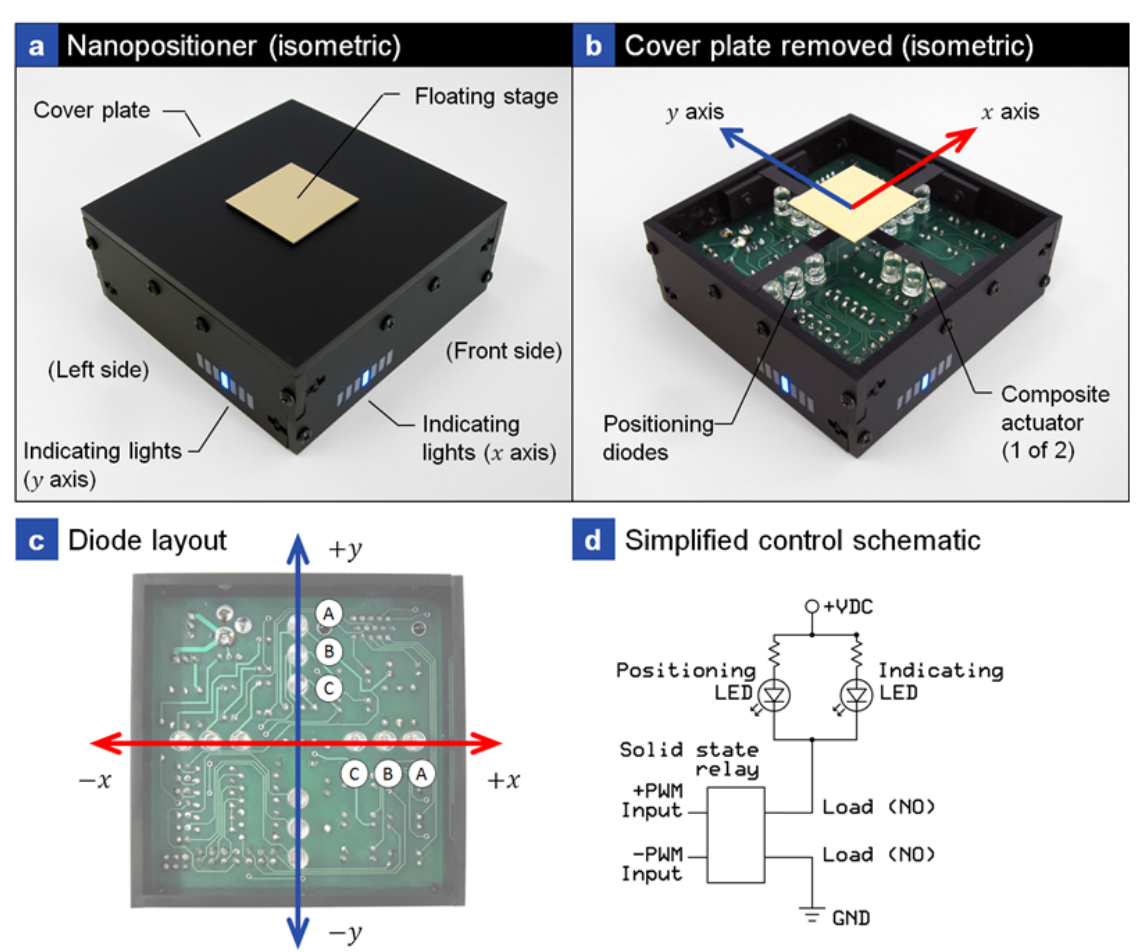

d Simplified control schematic

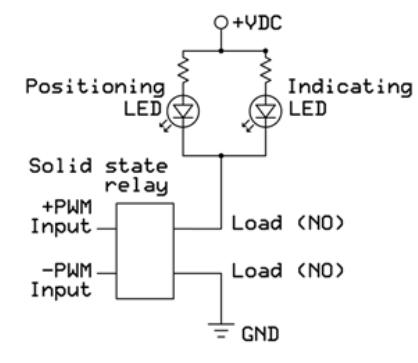

Figure 7.3. Nanopositioner overview. (a) Isometric of fully assembled nanopositioner. (b) Nanopositioner with cover plate removed, showing positioning diodes and dual GNP/PDMS composite actuators. (c) Top view of the nanopositioner with the actuators removed, clearly showing diode layout. (d) Simplified control schematic for a single positioning diode.

Figure 7.3(d) shows a simplified electrical schematic for a single positioning diode. A pulse-width modulation (PWM) signal drives a solid state relay that turns the positioning diode on/off. Visible wavelength indicating diodes from the position sensor serve as an operator feedback tool and are electrically connected in parallel and mounted externally on the nanopositioner to duplicate positioning diode intensity. To precisely control energy transduction to the polymeric chains and thus stage position, continuous high-speed intensity tuning of each diode is required. Therefore diode control for each 
axis was allocated to an independent PID controller, which was chosen due to ease of implementation and widespread industrial use. See Appendix F for discussion regarding PID control loop restrictions.

A control PCB allocates each IR LED to a dedicated 8-bit, $1 \mathrm{kHz}$ pulse-width modulation (PWM) channel yielding 256 IR intensity steps (value of 0 corresponded to $0 \%$ duty cycle, and 255 to $100 \%$ duty cycle). Assuming each IR intensity step corresponds to specific temperature change in the composite, this allows for 256 temperature steps. Figure 7.4(a)-(c) shows several plots of diode voltage at various duty cycles. LabVIEW-based control software was written to include modes ranging from manual control over each diode's intensity, to fully automatic, whereas the software determines diode adjustments required to position the stage. For the fully automatic mode, diode control for each axis was allocated to an independent proportional-integralderivative (PID) controller. PID feedback control was chosen due to ease of
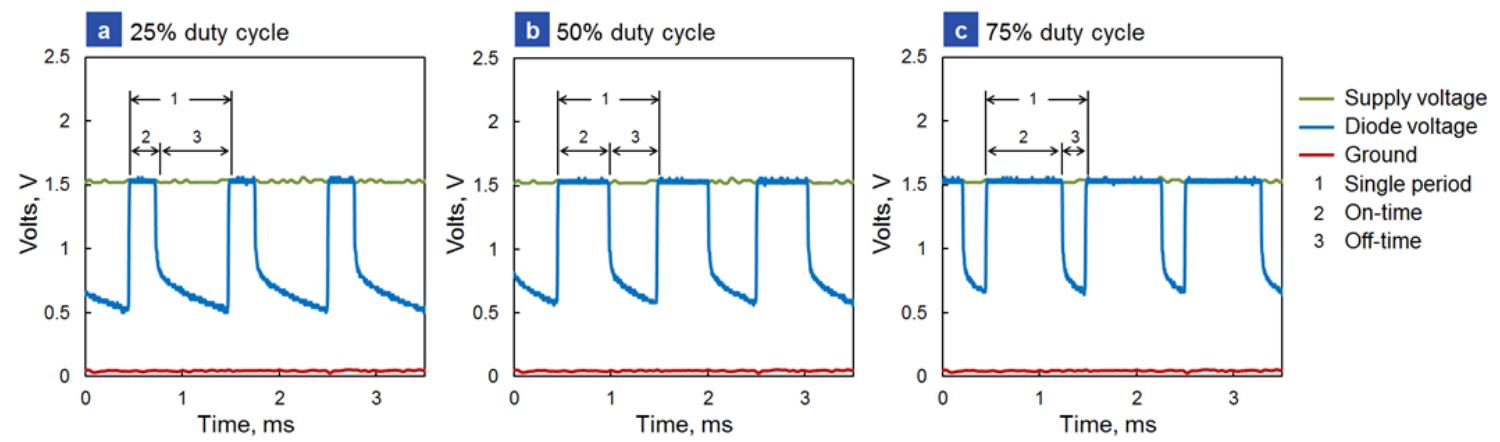

Figure 7.4. PWM signals. Using a benchtop Tektronix oscilloscope, supply voltage, diode voltage, and ground were monitored during routine nanopositioner operation. Plots of voltage versus time are shown for various duty cycles of the $1 \mathrm{kHz}$ PWM loop, including (a) $25 \%$ duty cycle, (b) $50 \%$ duty cycle, and (c) $75 \%$ duty cycle. Comparison of diode on-time (2) to off-time (3) is shown for a single period (1) on each plot. 
implementation and widespread industrial use. Control software allows the operator to input desired stage position $(x, y)$, laser displacement sensors provided real-time position feedback, and the PID loop sets diode intensity. Continuous high-speed diode intensity adjustments are required to account for thermal drift and maintain the stage at desired displacement.

\subsubsection{KINETICS OF PHOTO-THERMAL NANOPOSITIONERS}

The ability to dynamically modulate IR diode intensity results in a correlating temperature modulation in composite actuators and provides the mechanism for control of polymeric chain contraction/extension. As the number of intensity steps is fixed by the control system (at 256), the resolution of available temperature change steps is governed by the IR LED's maximum intensity specification. For example, if a high-power positioning diode ("coarse adjust") is replaced with a diode half as intense ("fine adjust"), temperature control resolution doubles, allowing for more accurate stage positioning. However, the increase in control comes at the expense of total stage travel. To quantitatively determine response differences between diodes, a maximum displacement test was conducted. This test involved measuring maximum possible stage displacement (first with all three positive, then all three negative diodes at 100\% duty cycle). The absolute values of the positive and negative displacements were averaged to determine overall response. Figures 7.5(a) and (b) present detailed displacement versus time data during a single run. 

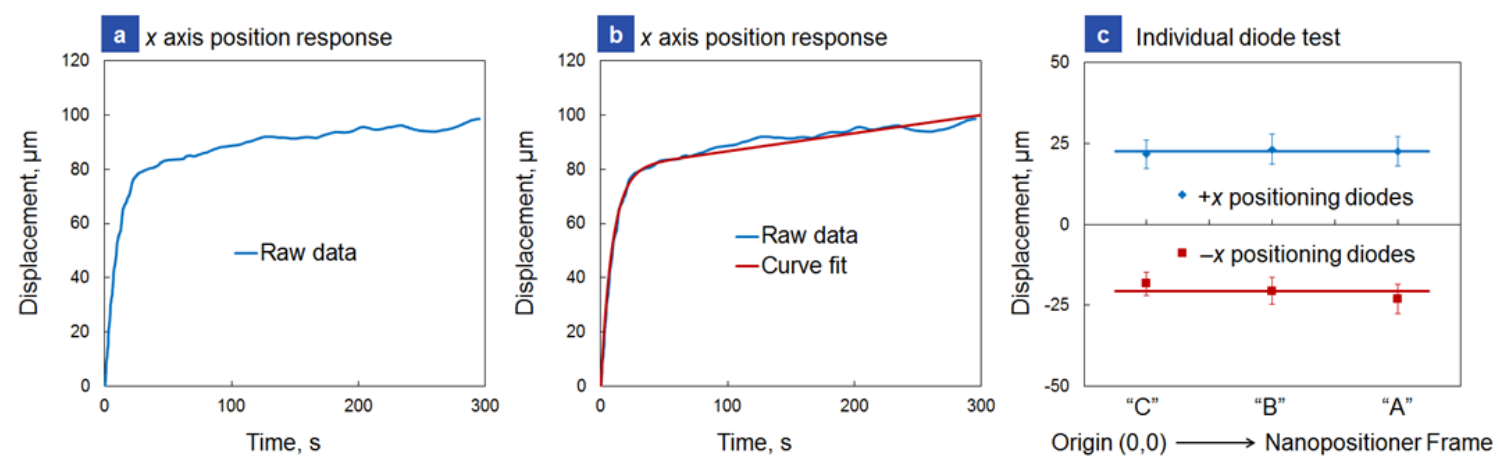

Figure 7.5. Kinetics curve fitting and individual diode test details. (a) Raw data is shown for a maximum displacement test ( $+x$ axis) on a $1 \mathrm{wt} \%$ GNP, $15 \mathrm{~s}$ spin time sample composite. At time $0 \mathrm{~s}$, duty cycle for all three $+x$ positioning diodes was set at $100 \%$. The curve shows stage displacement as a function of time. (b) After running each test, best fit curves were overlaid on top of the raw data $(\sigma=80 \mu \mathrm{m}, \tau=9 \mathrm{~s}$, and $\beta=15 \mathrm{~s})$. This process was repeated for the $-x$, then the positive axis and negative axis curve fitting coefficients averaged to determine normalized displacement kinetics for each composite skin and diode combination. (c) To determine individual diode contribution to the maximum stage displacement, this same process was repeated but with a single diode energized vice all three. The plot shows the individual contributions of each of the six diodes ( $1 \mathrm{wt} \%, 15 \mathrm{~s}$ spin time). Diodes labeled "C" are the physically innermost diodes [closest to the origin $(0,0)]$, while those labeled "A" are the outermost (closest to the nanopositioner frame).

Stage displacement from a single diode was verified to be $\sim 1 / 3$ of the maximum displacement [as Figure 7.5(c) shows], demonstrating actuation responses are confined to localized composite regions directly above each IR LED. Isolated response regions are a result of selecting positioning diodes with narrow beam angles as well as using GNP 
wt\%'s less than percolation threshold. Therefore, the total stage movement is a summation of all local responses, and each diode contributing to the overall response without affecting its neighboring diodes.

In order to determine stage kinetics, diode maximum displacement tests were conducted on various GNP wt $\%$ composites (of identical thicknesses). Figure 7.6(a) shows maximum displacement testing results as a function of GNP wt $\%$ loading for two different positioning diode types (coarse adjust, high-power IR diode and fine adjust, low-power diode). A discussion in Appendix G provides insight as to the advantages and tradeoffs of selecting high power vice low power IR diodes. While there was initially a sharp increase in maximum displacement with increasing GNP concentration, loadings beyond $\sim 0.75 \mathrm{wt} \%$ resulted in negligible additional stage travel demonstrating saturation of the photo-thermal response. The maximum displacement $\left(x_{\max }\right)$ responses as a function of GNP wt $\%$ were fit to equation (7.1) for both low power and high power diode, where $\delta$ is the displacement coefficient, and $\varphi$ the load adjustment factor. Figures 7.5(a) and (b) present detailed displacement versus time data during a single run. While high power IR diodes enable large actuator displacements, for a fixed number of intensity steps, lower power diodes allow for finer temperature control and resolution, resulting in more accurate stage positioning.

$$
x_{\max }=\delta\left[1-e^{(-w t \% / \varphi)}\right]
$$

Having witnessed the effect of high and low power diodes on maximum stage displacements, composites thickness was also found to affect overall stage kinetics. Figure 7.6(b) shows stage displacement responses for $90 \mu \mathrm{m}$ and $210 \mu \mathrm{m}$ thick 
composites (thinnest and thickest films made). Curves shown were fit to the raw data using equation (7.2), where $t$ is the time, $\tau$ is a time constant, and $\beta$ is a drift adjustment factor.

$$
x=\sigma\left[1-e^{(-t / \tau)}\right]+{ }^{t} / \beta
$$

Interestingly, thinner composites actuated faster, yet had lower maximum displacements than their thicker counterparts. With a fixed number of diode intensities available by our control setup, a composite with a larger displacement also had lower resolution. Conversely, while thinner samples have smaller maximum displacements, they also have finer control steps. The curve fitting in equation (7.2) to the experimental data show that the actuation and relaxation followed simple Debye characteristics and therefore a unique actuation mechanism ${ }^{54}$. Unlike CNT/PDMS actuators which follow a compressed exponential function for actuation ${ }^{54}$, GNP/PDMS actuators were found to not only follow a simple exponential function during both actuation and relaxation, but also exhibit about a three times faster response ${ }^{54}$. While for the same $\mathrm{wt} \%$ loading, photothermal amplitude of CNT/PDMS composites is larger than the GNP/PDMS system, however large amplitudes are only realized with efficient dispersion that results in high energy percolation within the composites. As CNTs are difficult to separate into individual nanotubes (vice bundles) and disperse uniformly within the polymeric matrix, CNT/PDMS composite fabrication is highly labor intensive with low yields making them expensive to produce. Conversely, GNPs are quite easy to disperse due to their rigid morphology, number of graphitic layers, and lack of adhesion or clumping between individual stacks. 


\subsubsection{USEFUL DISPLACEMENT VERSUS MAXIMUM DISPLACEMENT}

While curves in Figure 7.6(a) show maximum displacement as a function of GNP $\mathrm{wt} \%$, these reported values are steady-state equilibrium positions reached only after relatively long time periods ( $\sim 180 \mathrm{~s})$. While possible to achieve these displacements, due to long wait times required, they are not necessarily useful for practical nanopositioning applications. Therefore, additional criteria were set to determine useful displacement versus maximum displacement. This was achieved via selection of a tripwire value for slope of the displacement versus time curve. It was determined that when the displacement curve slope equaled 2 represented the best balance between (1) quick actuation times; (2) a large portion of maximum possible displacement; and (3) still allowed ample additional displacement control for maintaining the stage in position. Therefore, taking the derivative of the displacement [equation (7.2)], setting it equal to 2, and solving for time $(t)$ yields equation (7.3).

$$
t=-\tau \times \ln \left[\frac{\tau}{x_{\max }}(2-1 / \beta)\right]
$$

Applying this constraint to the plots in Figure 7.6(b), the displacement curve for the thin $(90 \mu \mathrm{m})$ composite sample reached a slope of 2 at $8.5 \mathrm{~s}$, representing a displacement of $\sim 47.2 \mu \mathrm{m}$ ( $\sim 50 \%$ of maximum displacement). For the thicker sample $(210 \mu \mathrm{m})$, a slope of 2 was reached at $14 \mathrm{~s}$, representing a displacement of $\sim 58 \mu \mathrm{m}$, or $\sim 60 \%$ of maximum. Thereby, a two-axis nanopositioner outfitted with the thin composite would have a total travel of $\sim 94 \mu \mathrm{m}$ per axis $(2 \times 47.2 \mu \mathrm{m})$, and outfitted with the thick composite would have a total travel of $116 \mu \mathrm{m}$ per axis $(2 \times 58 \mu \mathrm{m})$. Finally, actuation speed was calculated by dividing the usable displacement by the time required to reach 

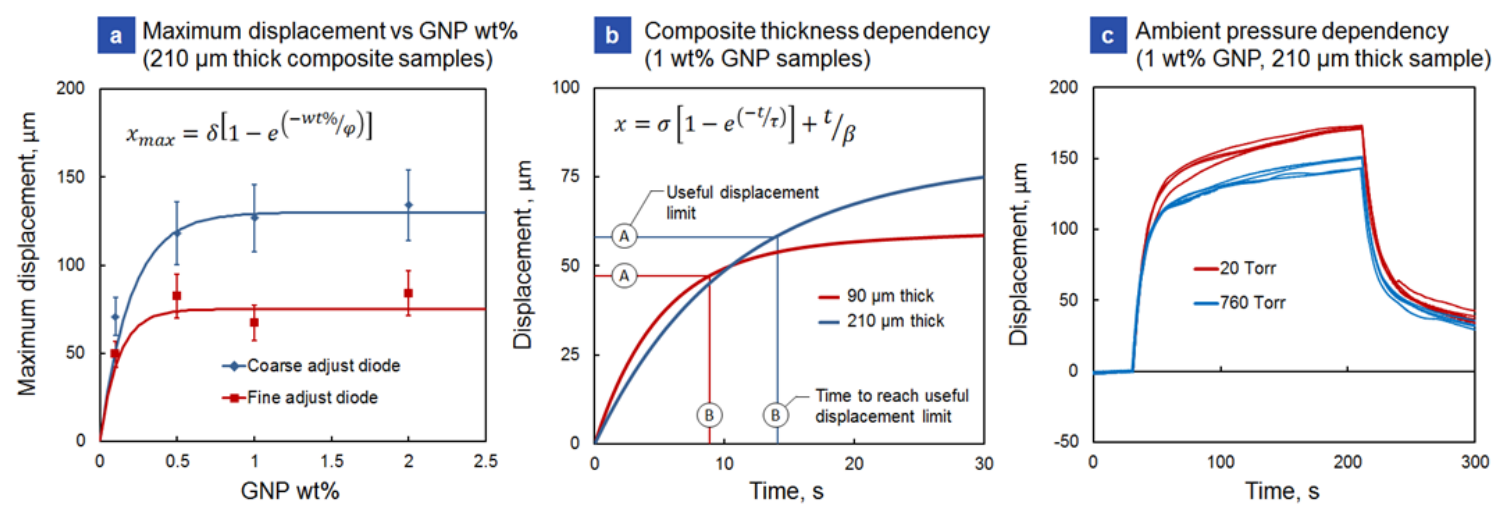

Figure 7.6. Positioning diode response characterization. (a) Maximum stage displacement as a function of GNP $w t \%$ loading for coarse adjust and fine adjust positioning diodes.

(b) Displacement kinetics as a function of composite actuator thickness for a $1 \mathrm{wt} \%$ GNP sample. Useful displacement limit and time are also indicated. (c) Displacement versus time curves shown for a $1 \mathrm{wt} \%$ GNP sample at ambient pressure (760 Torr) and at medium vacuum (20 Torr).

those values yielded velocities of $\sim 5.5 \mu \mathrm{m} / \mathrm{s}$ (90 $\mu \mathrm{m}$ "thin" composite) and $\sim 4.1 \mu \mathrm{m} / \mathrm{s}$ (210 $\mu \mathrm{m}$ "thick" composite). These velocity values can be improved significantly through use of much thinner composite actuators $(<1 \mu \mathrm{m})$. However, such ultra-thin composites will need new preparation steps as they are difficult to handle. One such solution is integration of thinner films onto MEMS substrates with on-chip positioning stages to improve photo-thermal actuation velocity.

\subsubsection{VACUUM DEPENDENCY OF PHOTO-THERMAL NANOPOSITIONER}

Many systems that utilize micro- and nanomanipulation technologies require operation in a vacuum. Therefore, in order to evaluate feasibility of photo-thermal nanopositioners as replacement actuation systems for work in vacuum environments, 
stage response pressure dependency was measured. Figure 7.6(c) presents displacement versus time curves for a $1 \mathrm{wt} \% 210 \mu \mathrm{m}$ thick composite sample at atmospheric pressure (760 Torr), as well as in a medium vacuum (20 Torr). Interestingly, at lower pressure (20 Torr), displacement was $\sim 30 \%$ higher than at atmospheric pressure, indicating as pressure decreases there is an associated drop in composite heat loss due to natural convection. Simply stated, as pressure is reduced, convective heat loss is minimized and identical diode intensity results in larger displacement. As pressure $(P)$ is reduced, density of the fluid ( $\rho$ ) follows, as equation (7.4) shows, where $R$ is the specific gas constant. Since the heat transfer coefficient is proportional to fluid (air) density, as pressure decreases, so does the heat transfer coefficient [equation (7.5)]. Since $\dot{Q}, A$, and $T_{\infty}$ remain constant, composite temperature must increase, as equation (7.6) shows. Since the pre-strained composite temperature is increasing, additional actuation is realized resulting in larger stage displacement under vacuum conditions. For future scaled-down photo-thermal systems that utilize actuators with submicron thicknesses, as ambient pressure decreases stage velocities are expected to increase due to lowered friction between gas molecules.

$$
\begin{aligned}
& \rho=P \downarrow / \overleftrightarrow{R T_{\infty}} \therefore \rho \downarrow \\
& h_{c} \propto \rho \therefore \rho \downarrow \\
& T_{\text {surf }}=\frac{\overleftrightarrow{Q}}{h_{c} \downarrow \overleftrightarrow{A}}+\overleftrightarrow{T_{\infty}} \therefore T_{\text {surf }} \uparrow
\end{aligned}
$$

While initial results are promising, further testing and characterization are required to determine nanopositioner suitability for use in high vacuum systems (such as 
in an SEM). Furthermore, one must consider that under vacuum, the positioning resolution of a given diode decreases (larger overall displacement for the same number of available PWM steps). Scaling effects also significantly affect heat loss versus heat generation in a vacuum environment at micro and nanometer length scales, and warrants further investigation. Finally, material properties of polymers and their outgassing rates should be determined before practical development of nanopositioners for vacuum environments. Therefore, vacuum-rated photo-thermal nanopositioners will require additional investigation before practical implementation to maintain the same performance characteristics as their ambient pressure-operated counterparts.

\subsubsection{ACCURACY AND RESOLUTION}

Nanopositioner ability to accurately respond to and maintain ordered positions was evaluated with $x$ and $y$ axes set to automatic (PID loop) control. Nanopositioners were given a sequence of ordered commands, and automated stage response monitored. Figures 7.7(a) and (b) show positioner response to a command sequence for $x$ and $y$ axes. Stage response (blue curve) is plotted against position command (red curve). Figures 7.7(c) - (e) show error between ordered and actual position with scale bars in $\mu \mathrm{m}$ and $\mathrm{nm}$ respectively. Large spikes are due to mismatch between actual and ordered positions upon new command receipt (annotations [1] and [2]). Figure 7.7(e), clearly shows the error spike [1] as the new position command is received, and the subsequent decay of the bounding oscillations (curves [3] and [4]). These oscillations although smaller than $120 \mathrm{~nm}$ are a result of filtering/averaging within the laser control head. While in reality, true resolution of the displacement sensor could be less than $120 \mathrm{~nm}$, the manufacturer's specification on the laser displacement sensor used for stage position feedback is $120 \mathrm{~nm}$, 

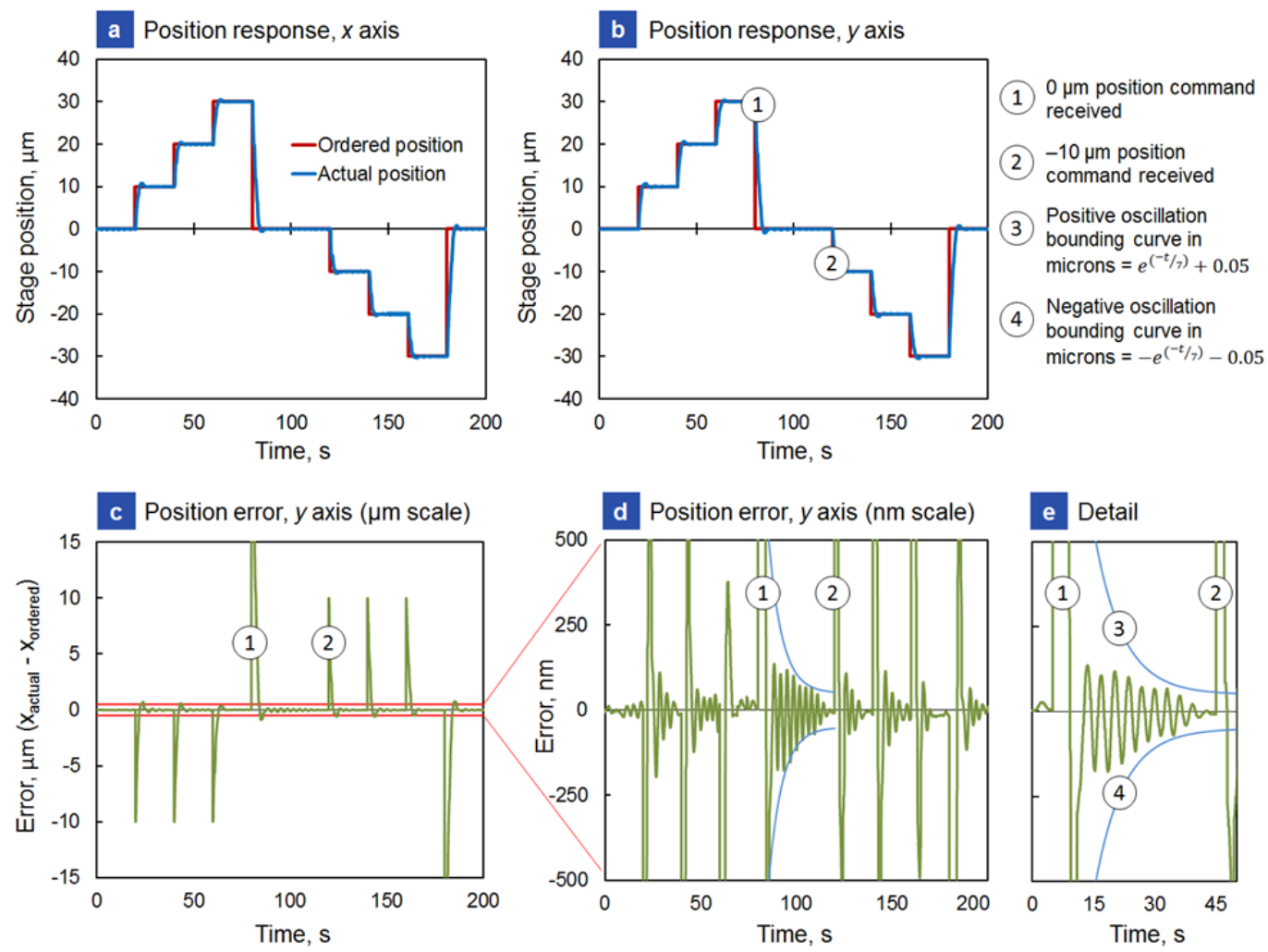

Figure 7.7. Stage response and stability in automatic control mode to position requests for both the $x$ and $y$ axes ( $1 \mathrm{wt} \%$ GNP composite). Ordered and actual (a) $x$ axis and (b) $y$ axis positions. Stage was ordered to sequentially move to positions $+10 \mu \mathrm{m},+20 \mu \mathrm{m}$, $+30 \mu \mathrm{m}$, and then $-10 \mu \mathrm{m},-10 \mu \mathrm{m},-10 \mu \mathrm{m}$. Annotations [1] and [2] indicate position commands for $0 \mu \mathrm{m}$ and $-10 \mu \mathrm{m}$ respectively. (c) Sample positioning error $\left(x_{\text {actual }}-x_{\text {ordered }}\right)$ with $\mu \mathrm{m}$ scale. Spikes are due to mismatch between actual and ordered position when the control loop receives a new position request. (d) Sample positioning error $\left(x_{\text {actual }}-x_{\text {ordered }}\right)$ with nm scale. (e) Detail of position response and subsequent stage oscillations. Positive and negative oscillation bounding curves are indicated by annotations [3] and [4] respectively. 
and therefore is considered the resolution limit for our nanopositioner. In follow-on generations, incorporation of capacitive displacement to monitor stage position (vice the current laser displacement system) can potentially increase positioner resolution to sub$10 \mathrm{~nm}^{15}$. Armed with increased resolution, stage positioning accuracy can be improved further through modifications such as (1) PID loop tuning, (2) incorporation of coarse adjust as well as fine adjust diodes into a single axis, and (3) higher frequency PWM control with a larger number of intensity steps.

Ideal for use in systems requiring precise positioning control/manipulation, such as probe stations and optical microscopes, photo-thermal nanopositioners were evaluated for use in several real-world tasks. For example, a nanopositioner was mounted to an optical microscope (Nikon Eclipse L150) for microsphere positioning. Figure 7.8 shows a sequence as the spheres are moved to positive and negative $x$ and $y$ axes displacement limits (arrows show the direction of movement). For clarification, a microsphere has been circled in red, clearly showing $\sim 100 \mu \mathrm{m}$ per axis photo-actuated positioning.

\subsubsection{ENERGY CONVERSION EFFICIENCY}

Graphene addition to elastomers not only creates high mechanical strength composites and photo-thermal actuation, but also could potentially be viable system for energy harvesting ${ }^{249}$. An important nanopositioner aspect is energy efficiency at converting IR illumination into $(x, y)$ stage movement. Therefore, efficiency $(\eta)$ of the GNP composites to a known IR illumination source was evaluated. Figure 7.9(a) presents the efficiency relationship as a function of GNP loading. Interestingly, a slight increase in efficiency with increased GNP loading was found, which ranged from $\sim 0.027 \%$ 


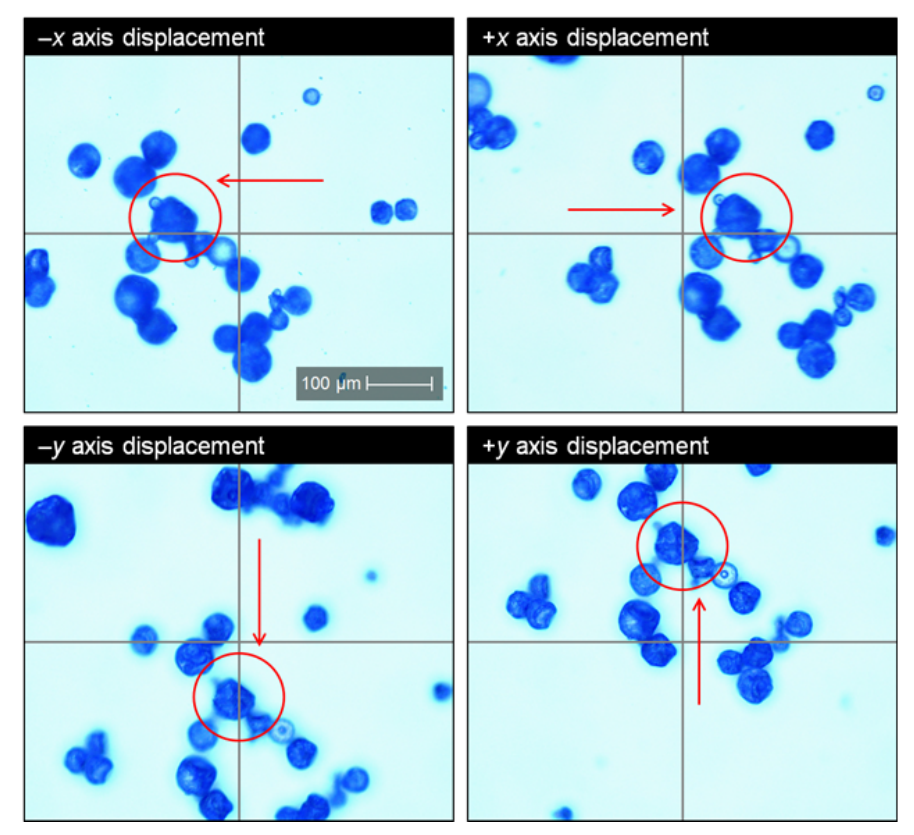

Figure 7.8. Actual stage response under optical microscope. Sequence of four images show the nanopositioner moving microspheres along the $-x,+x,-y$, and $+y$ axes. A single microsphere is circled in red, and crosshairs have been added for clarity.

( $0.1 \mathrm{wt} \%$ GNPs) to $\sim 0.033 \%$ ( $2 \mathrm{wt} \%$ GNPs). Efficiency calculation details can be found in Appendix H. Since graphene-elastomer photo-thermal actuation system is an example of a heat engine, measured efficiency was compared to maximum possible Carnot efficiency, $\eta=1-{ }^{T} / T_{H}$. While an identical IR illumination source was used, due to varying GNP loadings in the composites different $T_{h}$ temperatures were realized. For example, a $0 \mathrm{wt} \%$ GNP sample (optically clear ${ }^{190}$ ) experienced negligible temperature change, while $2 \mathrm{wt} \%$ GNP samples showed $\sim 50{ }^{\circ} \mathrm{C}$ change. Because of the larger temperature change, higher wt $\%$ composites had a larger maximum possible Carnot efficiency. Likewise, they also did more work and had a larger measured efficiency than their lower wt $\%$ counterparts. However, the looming question was - did the higher wt $\%$ 
composites use a larger portion of the available Carnot efficiency? Therefore, a ratio of measured to Carnot efficiency was determined [equation (7.7)] and simplified [equation (7.8)].

$$
\begin{aligned}
& \eta_{\text {ratio }}=\frac{\eta_{\text {measured }}}{\eta_{\text {carnot }}}=\frac{(W / Q)}{\left(1-T_{C} / T_{H}\right)} \\
& \eta_{\text {ratio }}=\frac{W T_{H}}{Q\left(T_{H}-T_{C}\right)}
\end{aligned}
$$

As Figure 7.9(b) shows, while just a small amount of GNPs $(0.1 \mathrm{wt} \%)$ resulted in a small composite temperature change, it was most efficient at transduction of this temperature change into useful work. By 0.5 to $2 \mathrm{wt} \%$, the $\eta_{\text {ratio }}$ was approximately equal. To summarize, incorporation of a small amount of GNPs into a composite results in an actuator that uses the largest percentage of maximum possible efficiency, this low $\mathrm{wt} \%$ composite also results in the smallest amount of work in response to the same IR illumination source. In comparison, past-light driven actuators based on PVDF have demonstrated an efficiency value of $\sim 8.3 \times 10^{-5} \%$ under quasi-static motion ${ }^{199}$, about three orders of magnitude less efficient than our graphene-elastomer systems $\left(\sim 3 \times 10^{-2} \%\right)$. Furthermore, improvements in efficiency for graphene-elastomer composites might be possible by using single layer graphene fillers and nanoscale thickness composite actuators. 

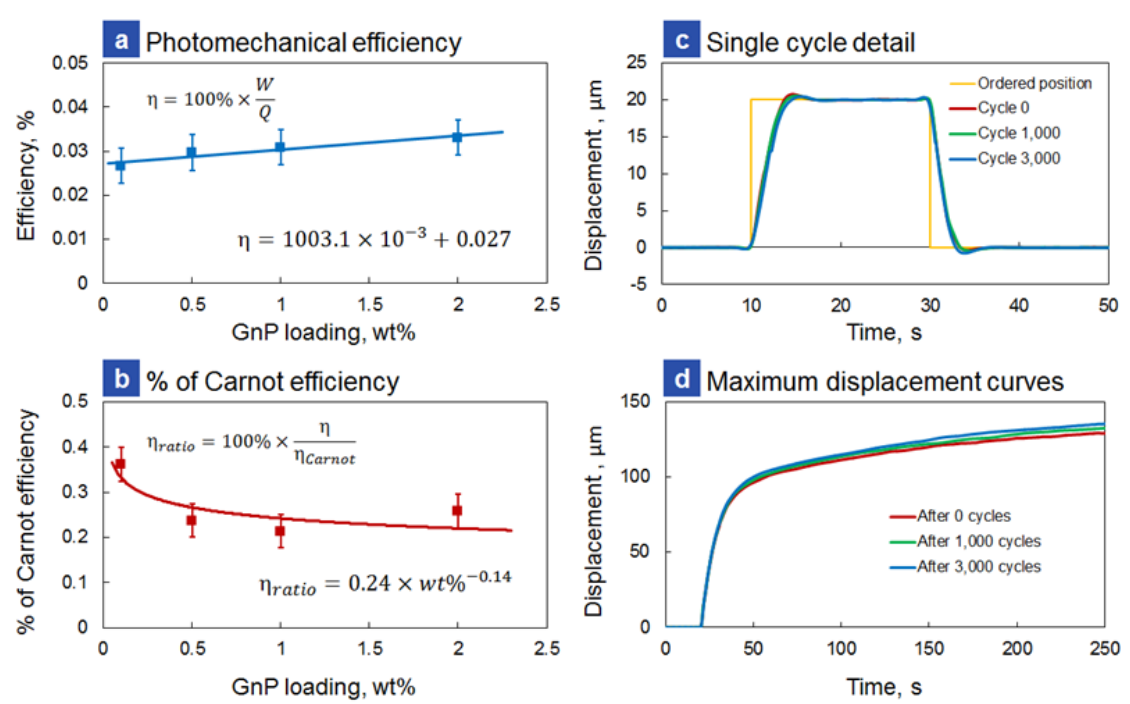

Figure 7.9. Photo-thermal efficiency and long-term degradation. (a) Photo-thermal efficiency versus GNP loading. (b) Photo-thermal efficiency divided by Carnot efficiency. (c) Stage position versus time curves are plotted for 0 cycles, 1,000 cycles, and 3,000 cycles. (d) Maximum displacement curves shown were conducted before the $1^{\text {st }}$ cycle, the $1,000^{\text {th }}$ cycle, and the $3,000^{\text {th }}$ cycle.

\subsubsection{LONG-TERM CYCLING AND COMPOSITE ACTUATOR DEGRADATION RESPONSE}

Experiments were also conducted to determine composite degradation as a function of repeated cycling. Figure $7.9(\mathrm{c})$ is a plot showing this $1^{\text {st }}$ cycle, the $1,000^{\text {th }}$ cycle, and the $3,000^{\text {th }}$ cycle. In order to monitor composite degradation, every 250 cycles the positioner performed a maximum displacement test. Figure 7.9(d) show the results of a maximum displacement test after $0,1,000$ and 3,000 cycles. As these plots show, even after 3,000 cycles, there was minimum-observed degradation, which demonstrates high lifetime of composite actuators. Appendix I provides detailed steps for all automatic testing sequences. 


\subsection{CONCLUSIONS}

This work introduces a new class of nanopositioners that exploit photo-thermal actuation mechanism in graphene-elastomer composites. Compared to electro-mechanical transduction, photo-mechanical/photo-thermal actuation offers an alternative way to couple energy into actuator structures offering distinctive advantages such as wireless actuation, remote controllability, electrical-mechanical decoupling, low noise, and scalability through existing MEMS processing technology. Unfortunately, few material systems have been shown to exhibit photo-mechanical actuation properties and are often not compatible with complementary metal-oxide-semiconductor (CMOS)/MEMS processing techniques. For example, photo-mechanical actuation has been achieved in PVDF $^{193}$, azobenzene ${ }^{194}$, shape memory polymers ${ }^{169}$, liquid crystal elastomers ${ }^{196}$, and chalcogenide glasses ${ }^{161}$. However, practical nanopositioner systems based on the previously mentioned technologies are rare. The system presented here is simple, lowcost, and could be integrated with myriad of systems such as optical microscopes and probe stations. Furthermore, numerous properties such as distribution of graphene networks, percolation threshold, elastomer matrix material, polymer chain segmental length, and composites thickness can be tuned to enable higher levels of control in future nanopositioning systems with a wide variety of application-specific tailorable parameters.

While at first it may seem that polymers are not an ideal material system for positioning applications due to thermal drifts, these drifts are easily corrected for using the differential dynamic photon-intensity modulation setup presented. Furthermore, ability to transduce photon energy into thermal energy that can change polymer chain mobilities at rapid pace to produce reversible actuation makes this system interesting to 
study. Tunability of $\mathrm{sp}^{2} / \mathrm{sp}^{3}$ graphene bonds in polymer composites could potentially result in extraordinary research systems with high mechanical strength, high intrinsic thermal conductivity, excellent electrical properties, and fast photo-thermal actuation. The uniqueness of our system - ability to dynamically tune IR intensity/temperature to change mechanical stiffness of the polymer chains in localized composite areas - makes this nanopositioner highly internally dynamic. One drawback of this work is that resolution of the feedback system limits the nanopositioner resolution to $\sim 120 \mathrm{~nm}$, however this is still better than the $\sim 170 \mathrm{~nm}$ resolution recently reported for a polysilicon-based thermal nanopositioners ${ }^{250}$. Current photo-thermal nanopositioner resolution limitations are due to the feedback system employed, not the actuators themselves. Hence next generation devices could incorporate capacitive as well as interferometric feedback resulting in much finer control, and achieve sub-10 nm resolution similar to recently demonstrated on-chip electro-thermal nanopositioning systems $^{251}$. Further development of graphene/polymer composite-based nanopositioners will extend the promising potential of graphene-based actuation technologies and will serve as a catalyst to inspire continued research into energy efficient transduction systems.

Thus far, this dissertation has dealt with characterization and applications of optically-driven carbon nanostructure/elastomer composite systems. While the preceding chapters have certainly established carbon nanostructure-based composites as an interesting and relevant research area, they were lacking an accompanying theoretical model. Therefore, using the associated stiffness increase of a pre-strained composite in 
response to IR illumination as the mechanism, Chapter 8 presents development of a corresponding spring-mass photo-mechanical actuation model. 


\section{CHAPTER 8 \\ SPRING-MASS PHOTO-MECHANICAL MODELING}

\subsection{BACKGROUND}

To round out the experimental characterization presented thus far in this dissertation, this chapter presents derivation and evaluation of a spring-mass model to describe photo-mechanical actuation in a single-axis nanopositioner. A number of simplifying assumptions are used; the most important being that temperature effects within the polymer composites are assumed to be the dominate mechanism responsible for actuation, and thus are the only ones considered in this model. Comprised of nanocarbons dispersed within a PDMS elastomer matrix, polymer-based photo-mechanical actuators are fabricated into thin films and subsequently cut into composite strips. After polymerization, but prior to use as actuators, composite strips are pre-strained. Composite deformation results in stretching of the polymer network chains, thus reducing system entropy ${ }^{247}$. Retractive forces are generated as a result of system tendency to seek the maximum entropy value found in the initial unstrained state ${ }^{247}$. Photo-mechanical actuation exploits control over this retractive force through temperature modulation. Temperature increases within the strained polymer composite add to the chaotic motion of the molecular chains, thereby further increasing system tendency to seek a more random configuration ${ }^{247}$. This results in either (1) a decreasing length of the polymer composite (when subjected to a constant force); or (2) increasing 
the retractive force (when composite is at a fixed length) ${ }^{247}$. The simplified models presented here are based on a Gaussian distribution, single-chain model (which was chosen due to characterization of long chains that behave as linear springs) ${ }^{247}$. The polymer chain spring constant is given by equation (8.1), where $K$ is the spring constant, $k$ is the Boltzman constant, $T$ is temperature (in absolute scale), $n_{b}$ is number of bonds, and $l$ is the bond length ${ }^{247}$.

$$
\begin{aligned}
& K=\frac{3 k T}{n_{b} l^{2}} \\
& K=\frac{3 \overleftrightarrow{k T}}{\left(n_{b} \downarrow\right)\left(\overleftrightarrow{l^{2}}\right)} \therefore K \uparrow
\end{aligned}
$$

Initial evaluation of equation (8.1) reveals two interesting relationships. First, since all factors on the right side of the equation are constants, $K$ is proportional to absolute temperature $(K \propto T)$. However, rather than merely evaluating the dependency of spring constant on temperature, comparison of thermal effects between a variety of different composites requires first normalizing responses. This can be done through calculation of the percent change in spring constant $(\% \Delta K)$ for a given temperature change $(\Delta T)$, which is proportional to $\Delta T$ divided by the original temperature (\% $\left.\% K \propto \Delta T / T_{0}\right)$. Figures 8.1(a) and (b) are graphs comparing theoretical and experimental $\% \Delta K$ for a $75^{\circ} \mathrm{C}$ temperature increase (starting with a $T_{0}$ of $40^{\circ} \mathrm{C}$, or $313 \mathrm{~K}$ ). A variety of GNP/PDMS composites (from 0 to $2 \mathrm{wt} \%$ loading) were individually mounted at $50 \%$ pre-strains (fixed at both ends) and placed in a thermal test chamber. Multiple thin-wire thermocouples within the chamber recorded temperature, and a load cell was mounted in series with one end of the composite for force measurements 
(converted to spring constants). Figure 8.1(a) shows the theoretical $\% \Delta K$ following a linear relationship with $\sim 0.32 \%$ increase in $\mathrm{K}$ per ${ }^{\circ} \mathrm{C}$. While also demonstrating linear relationships, experimental data nonetheless lagged the theoretical model, with only a $\sim 0.25 \%$ increase in $\mathrm{K} \mathrm{per}{ }^{\circ} \mathrm{C}$. With regards to the underperformance in experimental data however, there are several possible contributions. These include: (1) In experimental calculations, temperature values used for plotting $\Delta T$ at each point were generated from an average of all individual thermocouples readings. Depending on placement (i.e., center of chamber versus next to heating elements), however, thermocouple readings varied by as much as $\sim 15 \%$. (2) The thin-wire thermocouples used had much less thermal mass than the composite test samples, whose internal temperature (and thus change in spring constant) likely lagged slightly behind measured chamber ambient temperature. Furthermore, chamber temperature ramp rate was set at the minimum value of $\sim 10^{\circ} \mathrm{C} / \mathrm{min}$, however, lack of a "hold" feature prevented incorporating thermal stabilization wait times long enough to let composite spring response stabilize. Therefore, Figure 8.1(b) assumes a $\sim 20 \%$ lag in composite internal temperature (based on estimated combination of composite thermal mass effects and uneven chamber temperature distribution). Once adjusted for the lag, all three composite samples are almost identical to predicted theoretical response. As expected from equation (8.1), once normalized, carbon loading levels in the composites were confirmed to not have an impact on $\% \Delta K$ (all three responses essentially identical regardless of $\mathrm{wt} \%$ ).

The second relationship revealed from equation (8.1) can be used to describe changes in spring constant as a function of nanocarbon loading. As reinforcement materials are added to a polymer matrix, the matrix can result in shorter chain lengths and 
corresponding stiffness increases ${ }^{252}$. Therefore, in revaluating equation (8.1) with temperature held constant but chain length (number of bonds, $n_{b}$ ) decreasing, a corresponding stiffness increase is realized; equation (8.2) shows trends. Figure 8.1(C) shows the decrease in $K$ as a function of increasing the chain length. Assuming an initial number of monometer units in plain PDMS as $\sim 1,000^{253}$, theoretical chain length relationships are shown for experimentally determined spring constants of $0-5 \mathrm{wt} \%$ GNP/PDMS - starting with plain PDMS (0 wt\%) at 1,000 bonds, and progressing to $5 \mathrm{wt} \%$ at $\sim 500$ bonds (doubling of $K$ ).
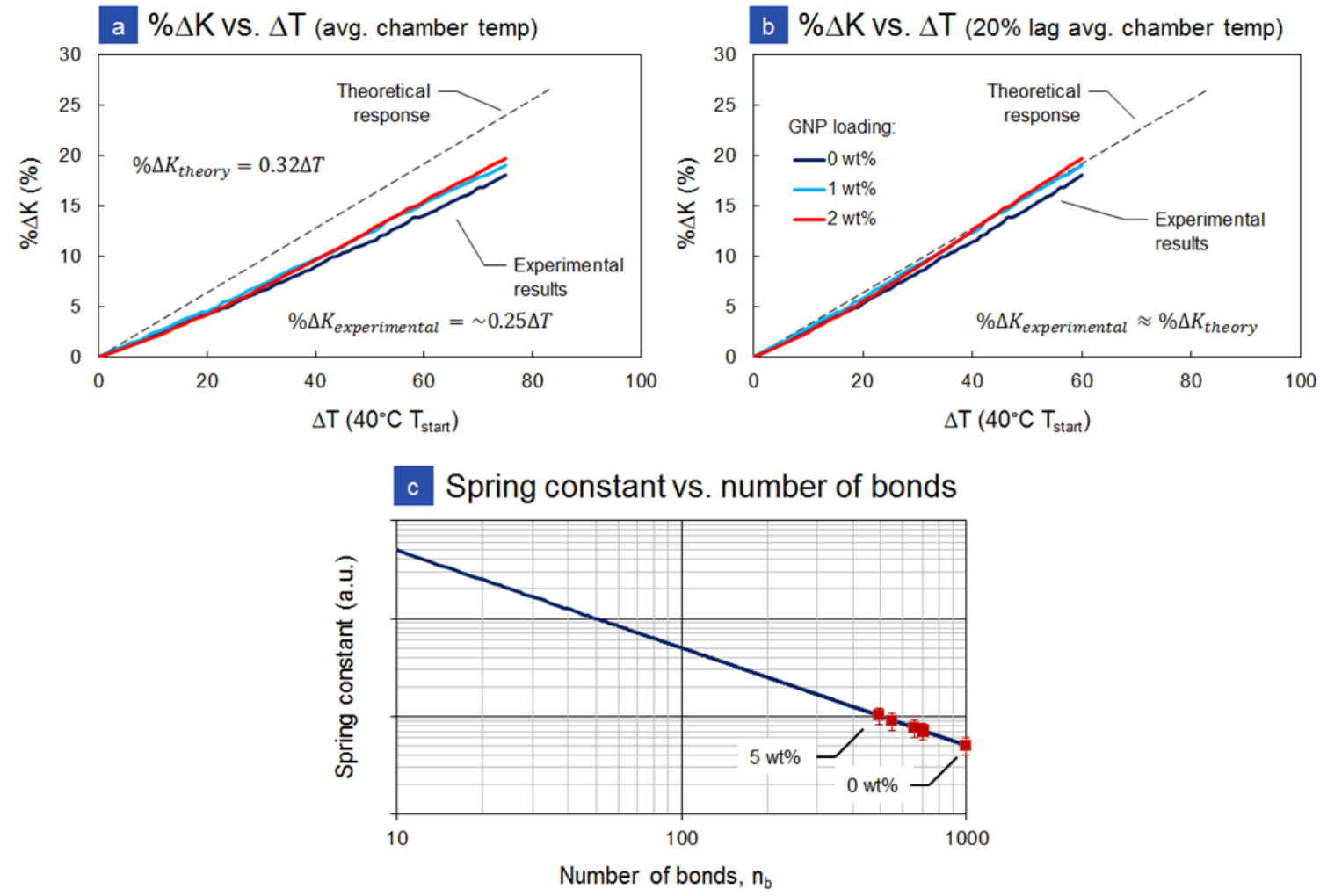

Figure 8.1. Spring constant relationships. Comparison between theoretical and experimental percentage change in spring constants as a function of temperature, using (a) average chamber temperature, and (b) 20\% lag chamber temperature. (c) Increase in spring constant as a function of reducing number of bonds in the polymer chain. 


\subsection{MODEL SETUP}

The real-world macroscopic composite actuators utilize PDMS rubber as a matrix, and therefore can be modeled as a simple linear spring [Figure 8.2(a)] of initial length $L$ and an effective spring constant $K_{\text {eff. }}$ For use in nanopositioning systems, the spring is pre-strained $(\varepsilon)$ with both ends held in a fixed position [Figure 8.2(b)].

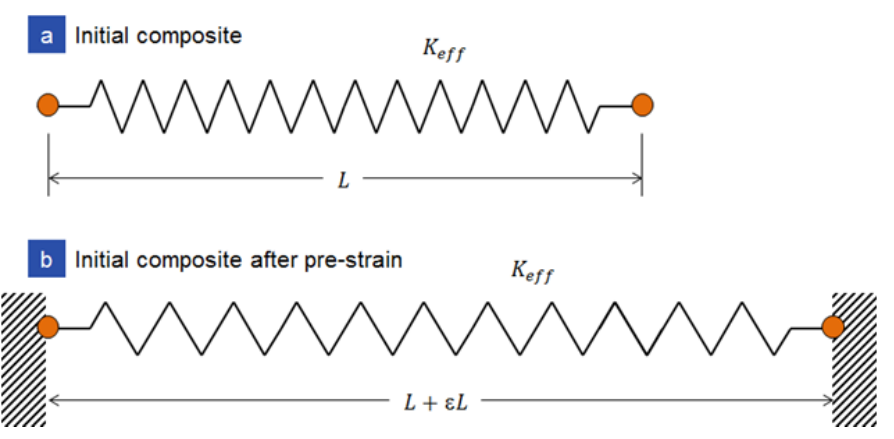

Figure 8.2. Initial system model. (a) Initial composite represented by a linear spring. (b) Initial composite spring shown pre-strained and fixed in nanopositioner housing.

Photo-mechanical actuation is unique as compared to other types of actuators in that photo-mechanical actuation allows one to dynamically and independently alter spring constants in specific regions of a polymer composite strip. The number and size of the regions is limited only by the IR illumination sources and associated optics. Therefore, the single-spring pre-strained composite actuator modeled in Figure 8.2(b) can be reimagined as multiple independent spring elements in series, Figure 8.3(a) shows. More closely modeling actual real-world setup, Figure 8.3(b) shows the positioning stage (with mass $m$ ) mounted in the center of the strip, such that the stage bisects the composite into $n$ spring elements on the left, and identical $n$ spring elements on the right (total spring elements in the system $=2 n$ ). Each spring element corresponds to its own IR illumination 
source, has length $\frac{L}{2 n}(\varepsilon+1)$, and has its own independent spring constant. The model represented by Figure 8.4 makes two important assumptions. First, it assumes that the positioning LEDs are chosen with beam angles such that illumination of one LED affects only its composite region and never its neighbor's region. Second, the model assumes that contraction (or expansion) occurs uniformly across each spring element.

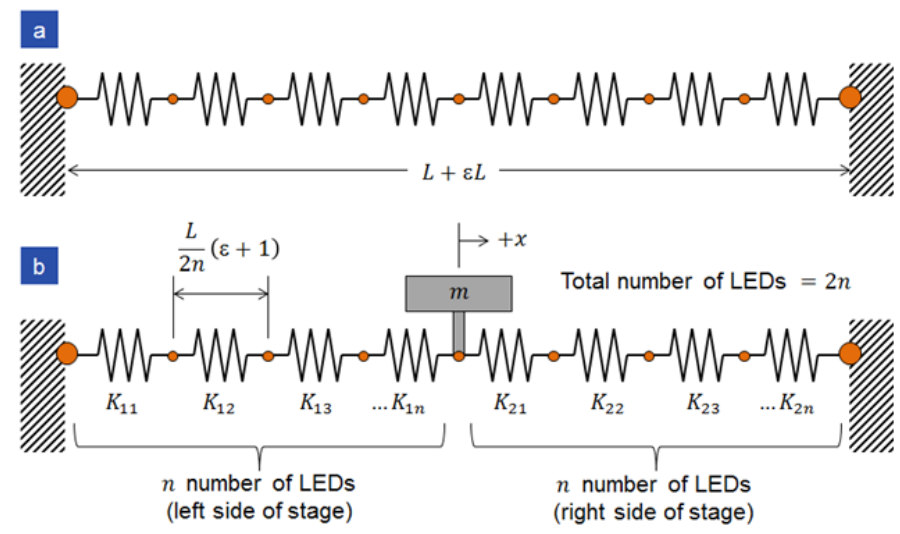

Figure 8.3. System model as multiple springs in series. (a) Division of initial composite into multiple springs in series. (b) Model with annotation indicating spring length, independent spring constants, and relationship of positioning stage to the springs.

Since the composite is pre-strained by some amount $\varepsilon$, this results in stretching each spring element by amount $\left(\Delta x_{\mathrm{i}}\right)$ [equation (8.3)] and an initial force $(F)$ [equation (8.4)]. Initially, all IR illumination sources are de-energized, resulting in identical spring constants in each element $\left(K_{\mathrm{i}}=K_{11}=K_{12}=\ldots K_{1 \mathrm{n}}=\ldots K_{2 \mathrm{n}}\right)$.

$$
\begin{aligned}
\Delta x_{i} & =\frac{\varepsilon L}{2 n} \\
F_{1 n} & =K_{1 n}\left(\frac{\varepsilon L}{2 n}\right)
\end{aligned}
$$




\subsubsection{EQUATIONS OF MOTION FOR INITIAL (NON-ILLUMINATED) SYSTEM}

For the initial, non-illuminated system, equation (8.5) shows the equation of motion. Due to all IR sources being de-energized and thus resulting in identical spring constants for each element, stage motion can be simplified, equation (8.6) shows. This simplification results in a natural system frequency of the stage, equation (8.7) shows.

$$
\begin{aligned}
& m \ddot{x}(t)=-x(t)\left[\frac{1}{K_{11}}+\frac{1}{K_{12}}+\cdots \frac{1}{K_{1 n}}\right]^{-1}-x(t)\left[\frac{1}{K_{21}}+\frac{1}{K_{22}}+\cdots \frac{1}{K_{2 n}}\right]^{-1} \\
& \ddot{x}(t)=-x(t)\left(\frac{2 K_{i}}{m n}\right) \\
& \omega=\sqrt{\frac{2 K_{i}}{m n}}
\end{aligned}
$$

Compared to other positioning systems such as piezoelectric actuators, the polymer composites utilized here have much lower stiffness values. While one would normally not associate a low stiffness polymer composite as an ideal material for use as an actuator, this property actually provides several potent advantages over its higher stiffness counterparts. For example, low stiffness offers a benefit as low stiffness results in an actuator with a much lower natural resonance frequency.

\subsubsection{EQUATIONS OF MOTION FOR RESPONSE TO IR ILLUMINATION}

Since each spring element can be independently controlled, system response to actuation of a single IR illumination source is evaluated. For the following examples, assume the IR LED affecting spring constant $K_{11}$ illuminates; Figure 8.4(a) shows the setup. First, a force balance equation around the stage is written [equation (8.8)]. Since 
only one LED is illuminated, only $K_{11}$ is affected. Substituting in Hooke's law and rearranging to isolate the element of interest on the left-hand side yields equation (8.9). The spring constants for all remaining spring elements will not be affected, and thus all equal $K_{i}$.

$$
\begin{aligned}
& -F_{11}+\sum_{i=2}^{n} F_{1 i}+\sum_{i=1}^{n} F_{2 i}=0 \\
& K_{11} x_{11}=\sum_{i=2}^{n} K_{1 i} x_{1 i}+\sum_{i=1}^{n} K_{2 i} x_{2 i}
\end{aligned}
$$

Since the system is anchored on both sides, an additional constraint can be applied in that the summation of all the elemental length changes must equal the original length [equation (8.10)].

$$
\sum_{i=1}^{n} x_{1 i}+\sum_{i=1}^{n} x_{2 i}=L(1+\varepsilon)
$$

Furthermore, since all spring elements except the one being illuminated are equal $\left(x_{12}=x_{13}=x_{1 n}=\ldots=x_{i}\right)$, equation (8.10) can be rewritten as equation (8.11), and solved for the general (non-illuminated) spring element displacement $x_{\mathrm{i}}$ [equation (8.12)].

$$
\begin{aligned}
& x_{11}+(2 n-1) x_{i}=L(1+\varepsilon) \\
& x_{i}=\frac{L(1+\varepsilon)-x_{11}}{2 n-1}
\end{aligned}
$$

Using these relationships to simplify equation (8.9) and solving for the IR illuminated spring length $\left(x_{11}\right)$ yields equation (8.13). 


$$
x_{11}=\frac{K_{i} L(1+\varepsilon)}{K_{i}+K_{11}}
$$

As established earlier, as a localized region of the composite section is illuminated, the spring constant $\left(K_{11}\right)$ in that region increases, while the non-illuminated spring constants $\left(K_{i}\right)$ are unaffected. Since the value of the denominator increases while the numerator is constant, the length of the spring $\left(x_{11}\right)$ decreases, equation (8.14) shows this trend. Being a doubly anchored system, however, the lengths of the remaining nonilluminated springs [as given by equation (8.12)] increase, equation (8.15) shows the trend.

$$
\begin{aligned}
x_{11}= & \frac{\overleftrightarrow{K_{l} L(1+\varepsilon)}}{\overleftrightarrow{K_{l}}+K_{11} \uparrow} \therefore x_{11} \downarrow \\
\mathrm{x}_{\mathrm{i}}= & \frac{\overleftrightarrow{L(1+\varepsilon)}-x_{11} \downarrow}{\overleftrightarrow{2 n-1}} \therefore x_{i} \uparrow
\end{aligned}
$$

Therefore, in response to IR illumination of a small composite region, the spring constant of that respective section increases causing a slight contraction. Since the macroscopic composite system is fixed on both sides, the rest of the composite stretches slightly to account for contraction in the illuminated region. Overall stage movement is a summation of individual contributions, as equation (8.16) shows. For the example given, the stage is moved to the left as shown in Figure 8.4(b). 


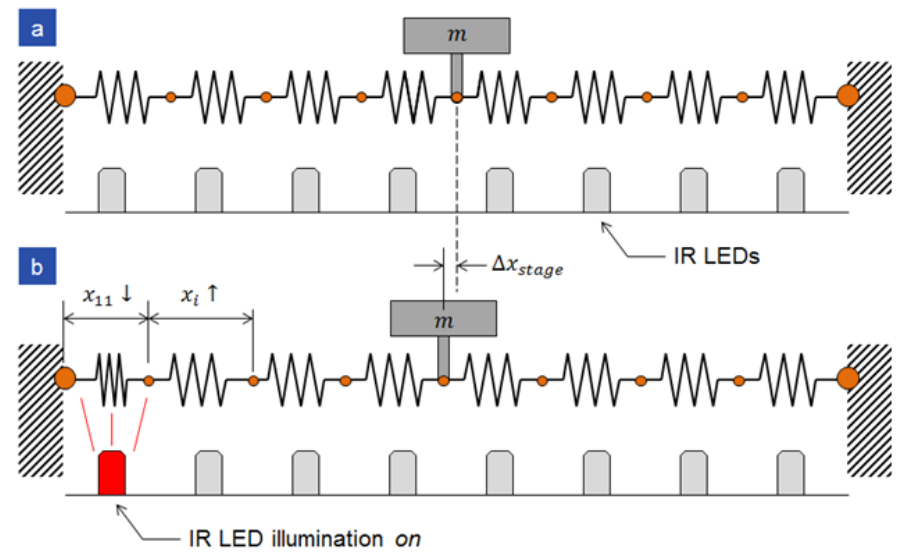

Figure 8.4. System response to IR illumination. (a) Relationship between spring elements and positioning LEDs (all LEDs off). (b) System response to single IR LED on showing element contraction and resulting stage movement.

$$
x_{\text {stage }}=-\sum_{i=1}^{n} x_{1 i}+\sum_{i=1}^{n} x_{2 i}
$$

By determining an effective spring constant for the elements on each side of the stage, the natural frequency of the stage for any illumination combination can be calculated [equation (8.17)]. Interestingly, this shows that the lowest natural frequency occurs in the completely non-illuminated state. As small areas of the composite are illuminated and individual sections stiffen, the natural frequency will always increase.

$$
\omega=\sqrt{\frac{\left[\sum_{i=1}^{n} 1 / K_{1 i}+\sum_{i=1}^{n} 1 / K_{2 i}\right]^{-1}}{m}}
$$

\subsection{RESPONSE TO ENVIROMENTAL TEMPERATURE CHANGE}

It is interesting to evaluate system response to environmental temperature changes. Assuming that ambient temperature $\left(T_{\infty}\right)$ change is moderately slow (as 
compared to ability to change control temperature using the positioning LEDs), both sides of the composite would experience the same temperature change; spring constants would follow suit, therefore, canceling each other out - resulting in no stage movement. One must keep in mind that since spring constant is proportional to absolute temperature [as given by equation (8.2)], however, a change in environmental temperature does affect ability to position the stage. Another way of expressing effect that ambient temperature plays on system response is to state that available change in spring constant $(\Delta K)$ is proportional to ratio of IR LED-induced change in temperature $\left(\Delta T_{\mathrm{LED}}\right)$ to ambient temperature. As ambient temperature increases, ability to dynamically alter the spring constants (and thus maximum available displacement) is adversely affected, as the trend arrows in equation (8.18) show.

$$
\Delta K \propto \frac{\overleftrightarrow{\Delta T_{L E D}}}{T_{\infty} \uparrow} \therefore \Delta K \downarrow
$$

\subsection{SPRING COMPRESSION AND STAGE MOVEMENT}

While equation (8.16) shows that stage movement is a summation of all individual segmented composite spring element displacements, not all contractions result in equal stage translation. To demonstrate importance of where contraction occurs in relation to the stage, a model identical to the real-world positioner is used (three IR LEDs per side, $n=3$ ) and shown in Figure 8.5(a). Assume a single LED is turned on to an intensity that causes $30 \mu \mathrm{m}$ of contraction. Since only a single LED is causing contraction, only about a third of the side (33\% spread) is contracting [as Figure 8.5(b) shows]. While the remaining composite regions stretch to account for this $30 \mu \mathrm{m}$ 
contraction $\left(\Delta x_{i} \uparrow\right)$, a significant amount of the stretching occurs on the same side of the stage as the contraction, thus canceling out some stage movement.

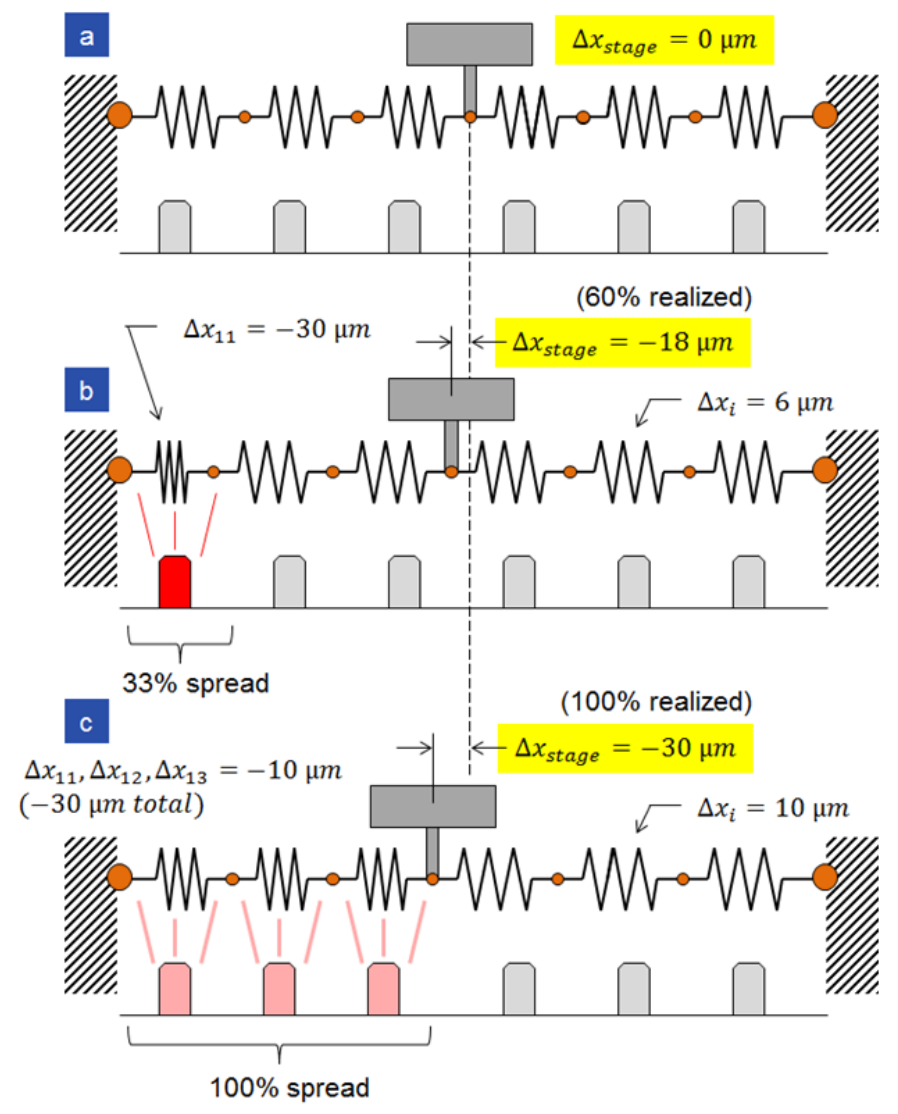

Figure 8.5. System response to IR illumination. (a) All LEDs off. (b) Single LED on, causing $30 \mu \mathrm{m}$ of contraction, resulting in $18 \mu \mathrm{m}$ of stage movement. (c) Three LEDs on at a lower power, yet still initiating the same $30 \mu \mathrm{m}$ of contraction, but resulting in $30 \mu \mathrm{m}$ of stage movement.

In this scenario, realized stage movement is $18 \mu \mathrm{m}$, only $\sim 60 \%$ of the $30 \mu \mathrm{m}$ generated contraction. Now consider that instead of a single high-intensity IR illumination signal, all three positioning diodes on the same side are illuminated at a lower intensity, subsequently causing only $10 \mu \mathrm{m}$ of contraction in each of their 
respective regions. There is still $30 \mu \mathrm{m}$ of contraction caused, however since this contraction is spread over the entire composite region on one side of the stage $(100 \%$ spread), all composite expansion is isolated to the opposite side of the stage and all $30 \mu \mathrm{m}$ is translated into realized movement [Figure 8.5(c)].

Figure 8.6(a) shows the resulting non-linear relationship between how much the IR-induced contraction is spread across a given side of the stage and the percent of that contraction that is realized as stage movement. While a relatively simple control loop was instituted for the two-axis positioner detailed in Chapter 7, this relationship illustrates a very powerful detail that can be exploited using a more exotic control setup. The profound implication of controlling where contraction occurs is that not only can a single stage be positioned, but rather multiple stages on the same actuator can be moved relative to one another. This is a very intriguing option when considering advanced object positioning and manipulation tasks. Further evaluating the effect of illumination spread on the spring constant, Figure 8.6(b) shows the percentage change in effective spring constant for the strip ( $\left.\% \Delta K_{\mathrm{eff}}\right)$ as a function of percentage of the overall composite actuator strip illumination. Intensity curves for $5^{\circ} \mathrm{C}$ to $75^{\circ} \mathrm{C}$ are shown. For example, if $\sim 50 \%$ of the composite is illuminated at an IR intensity that causes a $5^{\circ} \mathrm{C}$ temperature change, $K_{\text {eff }}$ of the overall composite will increase $\sim 2 \%$, but if the IR intensity is increased such that the same composite area increases $75^{\circ} \mathrm{C}$, then the spring constant increases by $\sim 13 \%$. 

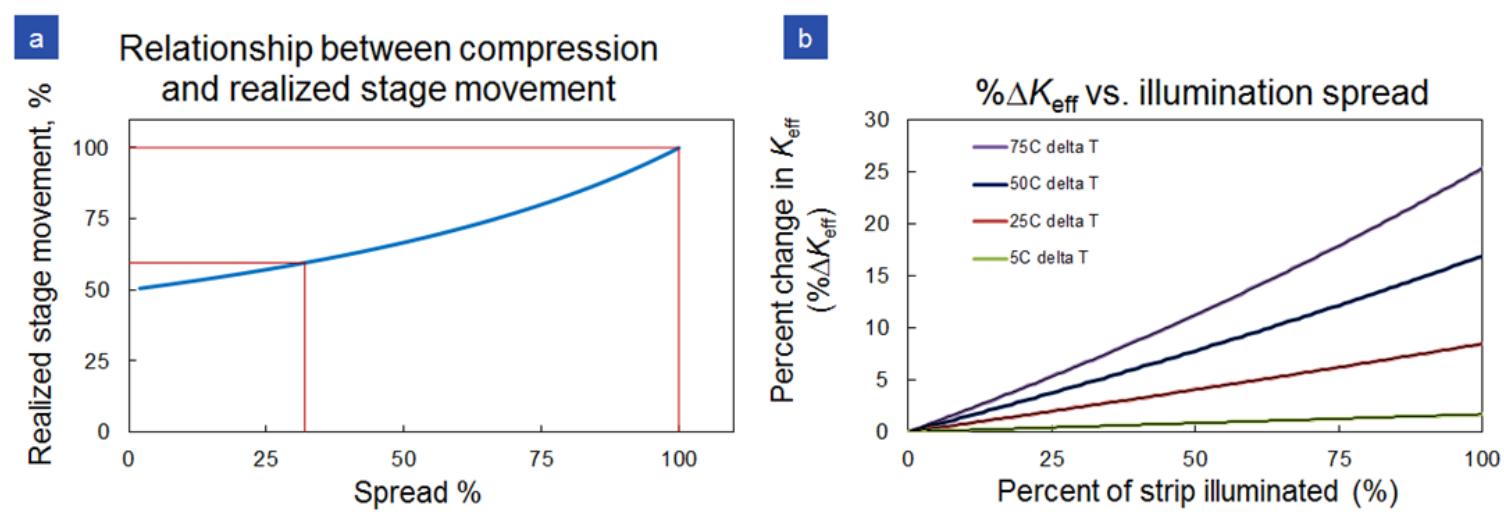

Figure 8.6. Illumination area to contraction relationships. (a) Relationship between percentage of the composite being illuminated (spread) and percentage of contraction that is translated to a stage mounted in the center. (b) Relationship between percentage of the composite is being illuminated (to a given temperature) and effective change in spring constant of the entire strip.

The ability to place multiple stages on a single polymer composite strip and position them independently from one another is a benefit not seen in any other type of positioning system, and warrants further investigation. Therefore, to help more fully demonstrate the vast array of positioning combinations available in a multi-stage photo-mechanical actuator; Figure 8.7 shows a simplified setup. The example shown consists of three positioning LEDs ( $\mathrm{LED}_{1}$ thru $\left.\mathrm{LED}_{3}\right)$ and two stages (1 and 2). The stages are mounted such that the pre-strained composite actuator is divided into thirds. Initially, with all LEDs de-energized, the system is at rest and both stages are in the home position [Figure 8.7(a)]. Using this as the starting point, we first examine positioning of a single stage only (Stage 2 to the left, Stage 1 to remain in the home position), as shown in Figure 8.7(b). In this example, $\mathrm{LED}_{2}$ is illuminated at a high intensity level, thus causing a contraction in its associated spring element. This will draw stage 2 to the left as desired. 
If no other LEDs are energized however, stage 1 will also reposition to the right rather than maintaining a desired static configuration. Therefore, low illumination of $\mathrm{LED}_{1}$ is also required to cause a slight compression in its spring element, thus canceling out the effect from $\mathrm{LED}_{2}$ and maintaining stage 1 stationary. Next, assume that rather than a single stage position, the operator desires to have both stages execute an identical movement to the left. Figure 8.7(c) shows the control combination required to accomplish this command, which includes $\mathrm{LED}_{1}$ illuminated at high intensity and $\mathrm{LED}_{2}$ at a lower intensity (to account for the effect from $\mathrm{LED}_{1}$ ). This will result in repositioning of both stages while keeping the same relative spacing between them. Finally, multi-stage photomechanical actuation also allows for positioning stages independently from each other, as Figure 8.7(d) shows. In this final example, only $\mathrm{LED}_{2}$ is illuminated, thus inducing movement in both stages - resulting in a change in relative positioning. For additional clarification, Table 8.1 gives a summary of (1) each LED intensity state; (2) change in spring length; (3) change in stage position, and (4) relative change in stage position for all four examples from Figure 8.7.

Table 8.1. Corresponding control system and spring response values for examples shown in Figure 8.7. Theoretical numbers are used to better illustrate positioning relationships.

\begin{tabular}{|c|c|c|c|c|c|c|c|c|c|}
\hline & \multirow{2}{*}{\multicolumn{3}{|c|}{ IR intensity }} & \multirow{2}{*}{\multicolumn{3}{|c|}{$\begin{array}{l}\text { Change in spring length } \\
\qquad(\mu \mathrm{m})\end{array}$}} & \multicolumn{3}{|c|}{ Change in stage position $(\mu \mathrm{m})$} \\
\hline & & & & & & & abs & lute & relative \\
\hline & $\mathrm{LED}_{1}$ & $\mathrm{LED}_{2}$ & $\mathrm{LED}_{3}$ & $\Delta \mathrm{x}_{1}$ & $\Delta \mathrm{x}_{2}$ & $\Delta \mathrm{x}_{3}$ & $\Delta \mathbf{x}_{\text {stage } 1}$ & $\Delta \mathrm{x}_{\text {stage } 2}$ & $\Delta \mathrm{x}_{\mathrm{rel}}$ \\
\hline (a) Initial setup & off & off & off & 0 & 0 & 0 & 0 & 0 & 0 \\
\hline $\begin{array}{l}\text { (b) Single stage } \\
\text { positioning }\end{array}$ & low & high & off & 0 & -20 & 20 & 0 & -20 & -20 \\
\hline $\begin{array}{l}\text { (c) Dual stage } \\
\text { positioning } \\
\text { (identical) }\end{array}$ & high & low & off & -20 & -20 & 20 & -20 & -20 & 0 \\
\hline $\begin{array}{l}\text { (d) Dual stage } \\
\text { positioning } \\
\text { (relative) }\end{array}$ & off & high & off & 10 & -20 & 10 & 10 & -10 & -20 \\
\hline
\end{tabular}




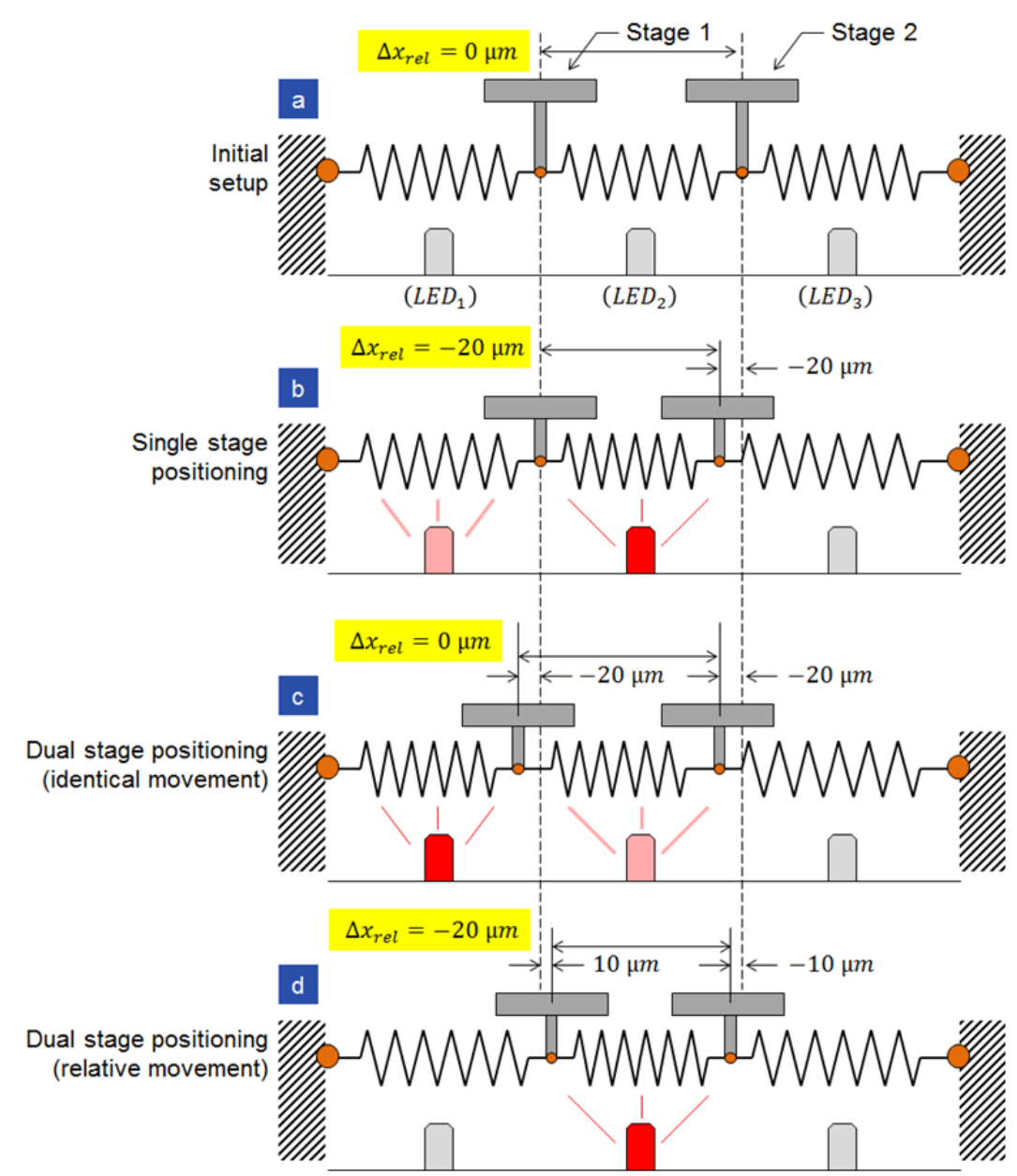

Figure 8.7. Multiple stages on a single actuation diagram showing (a) Initial setup, (b) Ability to position single stage only, (c) Ability to position both stages identically, and (d) Ability to position each stage independently.

\subsection{CONCLUSIONS AND FUTURE DIRECTIONS}

While there are a variety of improvements to consider for future generations of polymer-based photo-mechanical actuation systems, one of the main concerns lies with increasing the positioning resolution. First, ability to resolve finer movements is proportional to the number of illumination sources per side $(\propto n)$, and as $n$ approaches $\infty$ (and thus number of spring elements also approaches $\infty$ ), individual segment lengths 
approach zero. Second, for a fixed number of illuminated sources per side, resolution is also proportional to the number of different intensity states available for each source, with the ideal case being control over single photon emission. As the long bond lengths, wide bond angles, and low rotational barriers inherent to PDMS polymer result in high flexibility and internal mobility, the ultimate goal for photo-mechanical nanopositioning/manipulation is ability to control individual polymer chains with an overall 3D network.

Finally, several exciting possibilities arise when one considers exploiting some of the unique advantages that high resolution photo-mechanical actuation holds over more traditional types of positioning systems. While ability to move multiple elements independently on a single actuator (demonstrated previously) certainly presents exciting opportunities for nanomanipulation and fabrication, there is another characteristic of photo-mechanical actuation that is perhaps even more enticing. This second major advantage is the ability to actuate massive parallel arrays of photo-mechanical nanopositioners from a single illumination source. Currently, one of the main hindrances to widespread adoption of commercial nanomanufacturing is that one-at-a-time assembly/fabrication is time prohibitive (and therefore cost prohibitive) for anything other than one-off research applications. By harnessing the power of mass parallel actuation, however, batches of identical components can be fabricated using a single actuation array and single illumination control source. For example, using MEMS processing, arrays of millions of photo-mechanical nanopositioners can be created on a single substrate. In turn, a single illumination source/control system can be implemented to cause all of the positioners within the arrays to exhibit identical motion. Using this 
setup, one can envision applications such as mass fabrication of individual injectable cellular-sized capsules for controlled drug delivery, manufacturing swarms of identical micro/nanorobots, or using all the positioners in tandem to "knit" macroscopic-sized metamaterial samples.

Figure 8.8 shows one such approach for a mass array of photo-mechanical actuators. Starting off, a silicon wafer serves as the array substrate. Evaluating a small section of this wafer (1), an array of square cavities are lithographically patterned and then etched into the wafer (2). After creation of the cavities, thin pre-strained polymer composite strips are laid down such that they form a "tee" across each cavity (3). As with the nanopositioner systems demonstrated in this chapter, the "tee" layout allows for two-axis $x$ and $y$ positioning of each stage. In order to isolate actuation in one array element from affecting its neighboring array elements, the polymer composite strips are anchored to the cavity ridges (four anchor spots per array element). Finally, a stage is mounted to the center of each "tee".

While array fabrication is fairly straightforward, the question becomes how to implement a single illumination control source to create identical positioning responses in every array element. This feat can be accomplished by using an optical filter comprised of a lithographically patterned two-part custom mask in conjunction with a special illumination source. The illumination source required must allow for selecting between two different wavelengths, as well as two polarization settings for each wavelength. This combination allows for four uniquely addressable types of illumination, the minimum number required to achieve differential two-axis control $(+x,-x,+y$, and $-y)$. The twopart optical filter functions such that this type filter restricts the area of each array 


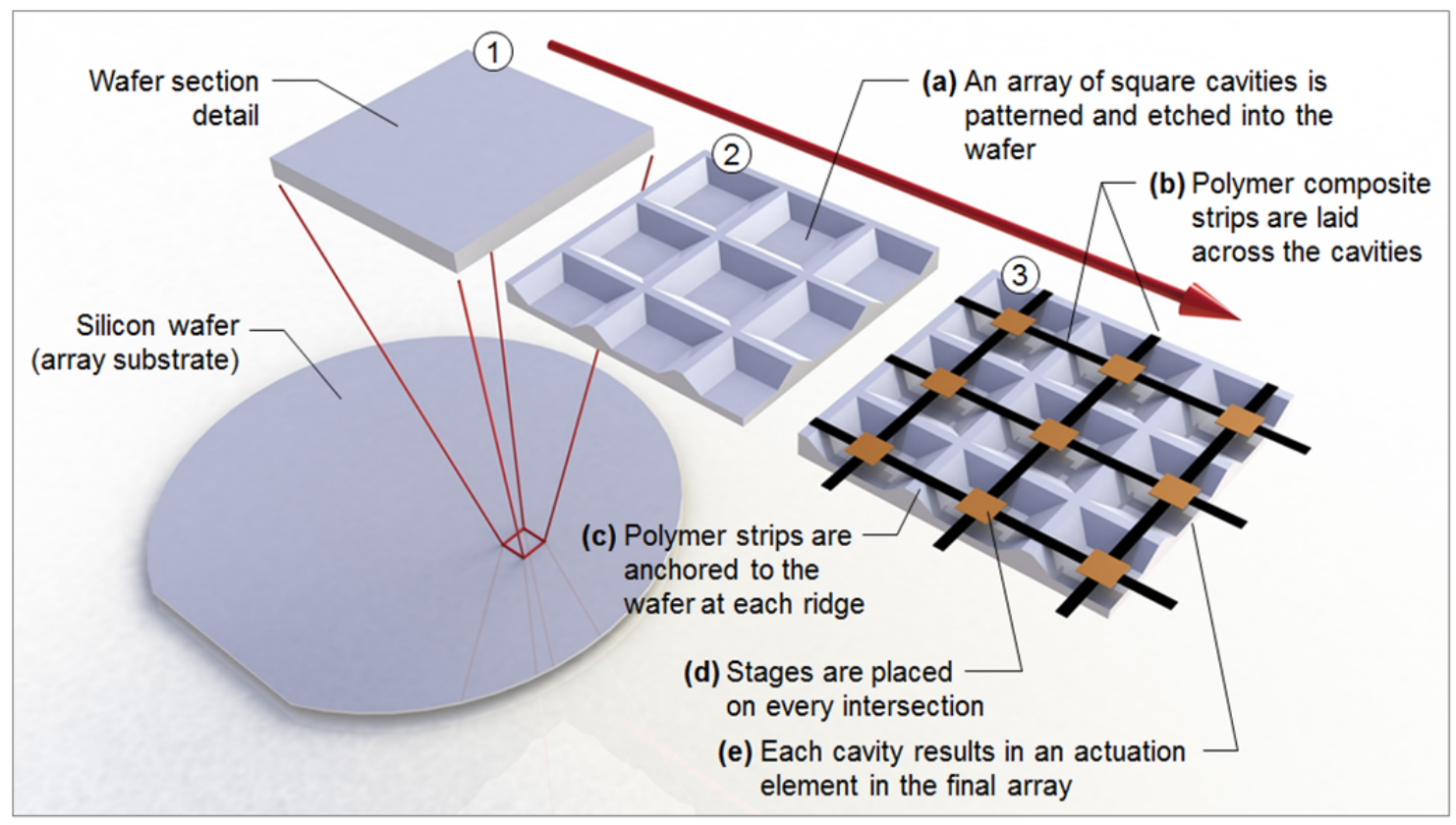

Figure 8.8. Actuator array fabrication sequence. Starting with a silicon substrate (1), an array of cavities is etched into the wafer (2). Thin polymer composite strips are prestrained and mounted to the cavities (3) resulting in an array of actuation elements.

element reached by the specific type of illumination. Figure 8.9 presents a model showing the physical orientation of the optical masks in relation to a small array element. In Figure 8.9(a), one can see that the two optical masks are placed over the array element. Figure 8.9(b) shows the top of the optical masks flooded with broad area illumination. As the illumination encounters the optical masks, however, only a small portion passes through to result in subsequent positioner actuation. By changing the illumination variables, four different actuation responses are possible. 
(a) Optical mask positioning

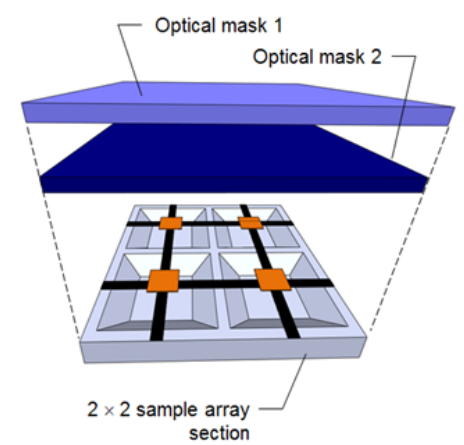

(b) Illumination response

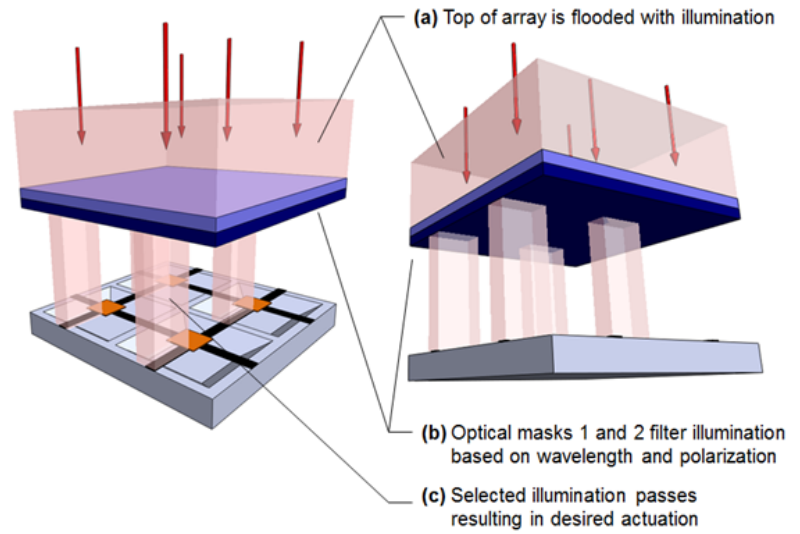

Figure 8.9. Optical mask positioning and array response detail. (a) Shows physical relationship of the optical masks to the actuation array. (b) Two views showing broad area illumination on one side of the optical mask assembly, and resulting filtered illumination that is allowed through (depends on polarity and wavelength).

Table 8.2 presents a summary of illumination variable combination and realized actuator position response. Figure 8.10 presents an overview of the optical mask patterns for a single array element. Figure 8.10 (a) is a top-view detail of a single two-axis array element. The first optical mask layer [Figure 8.10(b)] contains patterned regions of polarizing filter. The second optical mask layer [Figure 8.10(c)] contains two different band pass filter regions. When these two optical masks are used in conjunction to filter

Table 8.2. Summary of position response for all different combinations of illumination from the control source.

\begin{tabular}{ccc} 
Position response & Polarization angle & Wavelength \\
\hline$-x$ & $0^{\circ}$ & $\mathrm{A}$ \\
$+x$ & $0^{\circ}$ & $\mathrm{B}$ \\
$-y$ & $90^{\circ}$ & $\mathrm{A}$ \\
$+y$ & $90^{\circ}$ & $\mathrm{B}$
\end{tabular}


(a) Array element

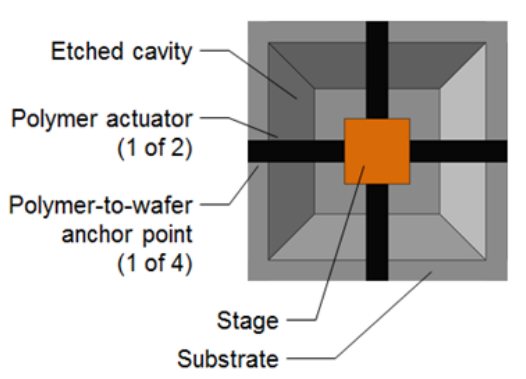

(b) Optical mask 1

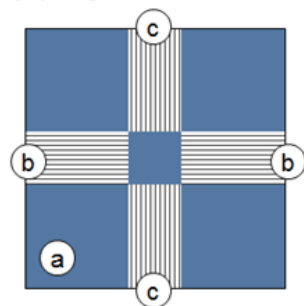

(a) Opaque region

(b) Polarized filter 1

(c) Polarized filter 2 (c) Optical mask 2

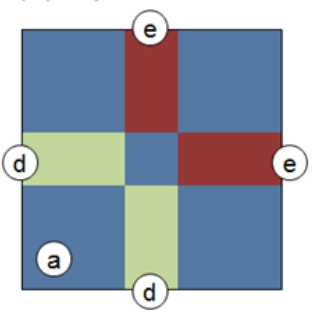

(d) Band pass filter 1

(e) Band pass filter 2 (d) $-x$ positioning

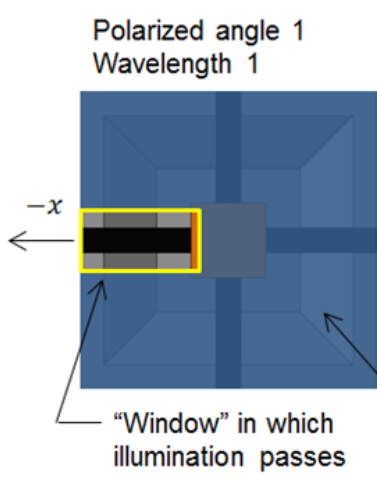

(e) $+x$ positioning

Polarized angle 1 Wavelength 2

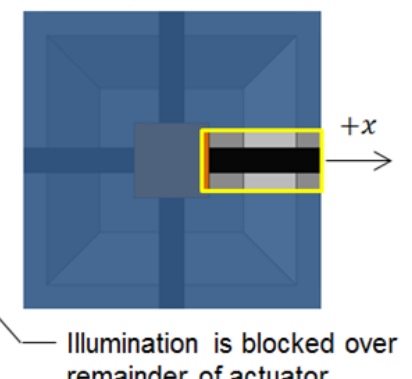

(f) $-y$ positioning Polarized angle 2 Wavelength 1

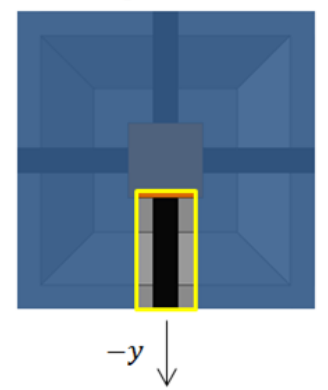

(g) $+y$ positioning

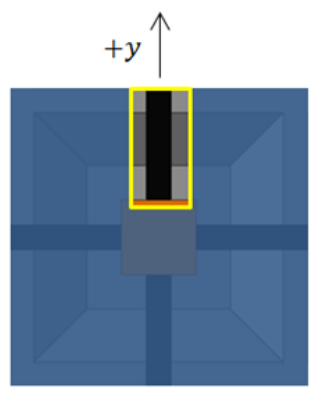

Polarized angle 2 Wavelength 2

Figure 8.10. Array control detail. (a) Single array element (top view). (b) Optical mask 1 showing regions of different polarity. (c) Optical mask 2 showing regions of different filtering. (d)-(g) show the "window" where illumination passes for each array element depending on the polarity and wavelength used by the control source.

broad area illumination, only one of four possible areas of the area element is actually illuminated. Because the optical filter mask patterns are arrayed identically to the positioner elements, identical motion response is repeated throughout the entire array. Figures $8.10(\mathrm{~d})$ thru (g) sequentially show for each of the four illumination combinations where the illumination passes and where it is restricted.

This chapter presented derivation of a mathematical spring-mass model to help predict photo-mechanical actuation responses. By modeling simple carbon/elastomer 
composite actuators as a series of springs (each with its own independently tunable $K$ ), insights into both single- and multi-stage positioning responses were achieved. The combination of this model with the experiment characterization of carbon nanostructure/elastomer composites from the previous chapters presents a well-rounded investigation into these novel optically-driven actuators. 
CHAPTER 9

CONCLUSIONS AND FUTURE RESEARCH

\subsection{CONCLUSIONS}

This dissertation was undertaken to investigate fundamental characteristics of photo-mechanical responses in pre-strained carbon nanostructure/elastomer composites and subsequently evaluate these responses for ultimate use in practical applications. Dispersion of various forms of nanocarbons into a PDMS elastomer matrix resulted in novel photo-mechanical actuation properties that showed reversible light-induced elastic expansion and contraction. Using a simple shear-mixing dispersion method, photomechanical responses of GNP/PDMS composites were first investigated. The study of GNP/PDMS composites included: (1) photo-mechanical stress response, Young's modulus, and actuation/relaxation kinetics in varying wt\% GNP loadings; (2) subsequent photo-mechanical stress comparison to several other nanocarbon forms; and (3) NIRinduced temperature change in the composites as a function of pre-strain. While the majority of photo-mechanical response is due to thermal effects, contributions may also arise from electrostatic, elastic, and polaronic effects. Solely using shear mixing dispersion for composite fabrication, photo-mechanical response in GNP/elastomer composites was 2.4 to 3.6 times larger than any other carbon form tested and significantly higher than for commercial polymers, such as PVDF, and azobenzene containing polymers and gels ${ }^{194}$. 
While the short-time shear mixing method was ideal for dispersion of GNP stacks within the polymer matrix, this mixing method proved to be a poor technique for other nanocarbons such as CNTs, which remained in relatively large bundles even in the fabricated composites. This called for an improved, or "evaporative mixing", dispersion method. This new method allowed for carbon/elastomer sample fabrication not previously possible with the shear mixing setup, and clearly demonstrated the critical importance nanocarbon dispersion within the polymer matrix plays on resulting photoinduced stress and efficiency responses. Carbon/PDMS composites were fabricated from four different carbon nanostructure dimensions [MWNT (1D), SLG (2D), GNP (2.5D), and HOPG (3D)] and previous experiment sets repeated, revealing interesting dimensionally dependent and percolation threshold dependent characteristics. For example, (1) decreasing dimensionality resulted in increasing photo-mechanical stress response and efficiency. The MWNTs (1D) tested were found to be the most effective nanocarbon for photon absorption and energy transduction to the polymeric chains, this relationship held at various wt\% loadings as well as illumination intensities. (2) As opposed to the dimensionally dependent photo-mechanical response, actuation and relaxation kinetics were found to be related to the carbon loading level in relation to the percolation threshold. Composites at $\mathrm{wt} \% \mathrm{~s}$ less than the percolation threshold (insulating region) responded with nearly identical actuation and relaxation times, regardless of dimensionality. However, composites at wt\%s greater than the percolation threshold (conducting region) responded not only slower for both actuation and relaxation, but also had a higher magnitude of photo-mechanical stress response - indicating that connective carbon nanostructure networks resulted in energy transduction and resulting 
photo-mechanical actuation beyond the point of illumination. (3) Photo-conductive responses in conducting composites were found to be both pre-strain and $\mathrm{wt} \%$ loading dependent. IR illumination of these samples resulted in decreasing resistance, which showed that e-h pair generation within the composite samples.

Photo-mechanical actuation of ternary systems was also studied. Analysis found that with addition of thermally expanding microspheres, one could extract macroscopic photo-actuation responses from the composites. Photo-induced microsphere expansion resulted in visible dimensional changes, regions of reduced polymeric chain mobility, nanotube tensioning, and overall elastic to plastic-like transformation of the composite, allowing for incorporation of sensing (through conductivity changes from CNT junction rearrangement) and actuation (microsphere expansion) into a single coherent functional material. Use of this method entails a significant drawback, however, namely that actuation responses are unidirectional. Even so, a plethora of important applications still exist, for example as pop-up structures, on-demand structural elements, and biomedical applications such as stents. Starting with simple CNT/microsphere/elastomer composites, important photo-mechanical response properties were investigated, including:

(1) Increasing photo-induced volumetric expansion with increasing microsphere loading;

(2) composite density reductions with increasing microsphere loading; (3) changes in composite CNT volumetric percent due to photo-induced expansion versus constant weight percent loadings; and (4) photo-induced composite Young's modulus increases as the microspheres expand, resulting in an elastic to plastic-like transformation of composite samples. Although microspheres cause overall system expansion, contractive actuation can still be attained, as evidenced by the demonstrations of selective patterning. 
Results from photo-mechanical response characterization in GNP/elastomer structures led to development and prototype of a two-axis sub-micron translation stage. The ability to transduce photon energy into thermal energy in the polymer composites and rapidly change chain mobilities was studied using a differential IR LED setup. A closed-loop feedback system allowed for precise thermal energy control, as well as allowed for construction and evaluation of a practical photo-mechanical nanopositioning system. Capable of controlled displacements of more than $100 \mu \mathrm{m}$ per axis, actuation speeds exceeding $5 \mu \mathrm{m} / \mathrm{s}$, sub-micron resolution, and low-cost, this system demonstrated a real-world application of graphene/polymer systems for nanopositioning. The unique photo-thermal positioning mechanism was achieved via dynamic modulation of multiple independent IR sources placed along the actuators that enabled differential control over polymeric chain stretching/contraction. Disposable composite actuators were fabricated using GNP loadings from 0-2 wt $\%$ and thicknesses from $90-210 \mu \mathrm{m}$, and exhibited longterm stability ( $>3,000$ cycles) and opto-mechanical efficiency of $\sim 0.03 \%$.

The photo-mechanical nanopositioning system further served as an inspiration for development of a spring-mass mathematical model. To better understand photomechanical responses in the composite actuators, thermal effects on polymer chain spring constants were evaluated. Nanocarbon/elastomer actuator strips were simulated as a series of $n$ spring elements, with each element having an independent spring constant. Assuming the dominant mechanism in photo-mechanical actuation is due to thermal influences, relationships between spring constant and IR-induced heating were derived and validated using experimental data. The simple model allowed for independently tuning each spring element's respective spring constant (via IR illumination), and thus 
resulting in ability to cause both contraction and expansion in specified actuator regions. Subsequent model analysis presented insight into effects of (1) ambient temperature changes on photo-mechanical response; (2) IR illumination intensity; (3) IR illumination area in relation to stage movement; and (4) percent of the overall composite actuator illuminated. Furthermore, this model provided a demonstration, using a control setup similar to that described in Chapter 8 , showing how multiple stages could be mounted onto a single composite actuator strip, resulting in (1) translation of a single stage, (2) multiple stage translation with identical displacement, and (3) multiple stage movement with independent translation. Significantly, these are features not present in any other actuation system, yet present tantalizing options for advanced nanopositioning and nanomanipulation tasks as well as represent a significant milestone in actuation control.

\subsection{FUTURE RESEARCH}

Future photo-mechanical research directions and suggestions are provided in this section. While this dissertation characterized photo-mechanical responses across a variety of carbon nanostructure forms, the underlying mechanisms behind photo-mechanical actuation are poorly understood. For purposes of modeling the spring-mass system, the primary vehicle behind actuation was assumed to be thermal, which by comparison with experimental testing was shown to be reasonably accurate; however, contributions from electrostatic, elastic, and polaronic effects may also be present ${ }^{52}$. Advanced experimental setups, which can isolate for each of these effects, are needed to determine individual contributions to overall holistic photo-mechanical actuation. Rather than the emphasis on macroscopic composite sample sizes tested here, future work should concentrate on 
responses within mesoscopic carbon/polymer chain samples. Using these samples, studies into the complication interactions taking place at the carbon/polymer chain interface may be possible. Furthermore, as demonstrated experimentally, important changes in photo-response occur when carbon loading in the sample crosses the percolation threshold forming a conductive network. Study of smaller samples will help illustrate effects occurring at this important boundary. Furthermore, as touched upon in the dissertation, tuning the $\mathrm{sp}^{2} / \mathrm{sp}^{3}$ graphene bonds in carbon nanostructures could help improve mechanical strength, thermal conductivity, electrical properties, and overall photo-thermal actuation responses.

The experimental characterization demonstrated in this dissertation could also be repeated using either pristine SLG (vice SLG derived from RGO), or with multi-carbon, mixed-dimensional systems. Due to lack of defects and edge effects between individual sheets, a single pristine graphene layer would result in faster thermal responses, and perhaps enhanced photo-mechanical actuation. For mixed-dimension systems, (1) thermal conductivity, (2) electron transport, (3) photo-conductivity, (4) thermoelectric power, and (5) optical absorption are expected to be different compared to their single carbon nanostructure counterparts, and be dependent on geometry, anisotropy, and orientation of the carbon additives. This presents a host of enticing possible properties for characterization and applications, such as (1) built-in electric fields due to difference in thermoelectric power, (2) controllable optical absorption characteristics [which could impact IR sensors ${ }^{215}$ ] and allow for photo-mechanical actuators with built-in optical filters, and (3) synergistic effects between carbons that reduce interfacial resistance for thermal conduction. Finally, properties such as distribution of graphene networks, 
tailoring the percolation threshold, and use of different elastomer materials could enable application-specific levels of control in future nanopositioning systems.

From an applications standpoint, it is easy to envision numerous applications that are more dynamic and specialized than the straightforward ones presented in this dissertation. For example, large area thin film skins, which exploit both dimensional and percolation dependent photo-mechanical traits of photo-mechanical actuation, could help replace traditional flight control surfaces. Choices between creating composites with carbon loadings less than the percolation threshold would result in locally addressable actuation areas, while conductive composites could exhibit wide-area deformation. Approaching applications from a biomedical aspect, already photo-mechanical actuation could have an immediate impact in lab-on-a-chip applications, flexible bioelectronics, and sensors. For future research into microscopic actuation (as demonstrated by the two-axis translation stage), on-board capacitive or interferometric feedback is required in order to achieve finer resolution and control. Further development of graphene/polymer composite-based nanopositioners will significantly extend the promising potential of graphene-based actuation technologies and will serve as a catalyst to inspire continued research into energy efficient transduction systems. 


\section{REFERENCES}

1 Otsuka, K. \& Ren, X. Physical metallurgy of Ti-Ni-based shape memory alloys. Progress in Materials Science. 50, 511-678 (2005).

2 Biener, J. et al. Surface-chemistry-driven actuation in nanoporous gold. Nature Materials. 8, 47-51 (2009).

3 Nemat-Nasser, S. Micromechanics of actuation of ionic polymer-metal composites. Journal of Applied Physics. 92, 2899-2915 (2002).

4 Jeong, B. \& Gutowska, A. Lessons from nature: stimuli-responsive polymers and their biomedical applications. Trends in Biotechnology. 20, 305-311 (2002).

5 Carazo, A. V. \& Uchino, K. Novel piezoelectric-based power supply for driving piezoelectric actuators designed for active vibration damping applications. Journal of Electroceramics. 7, 197-210 (2001).

6 Sakamoto, M., Fujistuka, M. \& Majima, T. Light as a construction tool of metal nanoparticles: synthesis and mechanism. Journal of Photochemistry and Photobiology C-Photochemistry Reviews. 10, 33-56 (2009).

$7 \quad$ Wang, C. C. \& Roy, S. Energy and force prediction for a nanosecond pulsed dielectric barrier discharge actuator. Journal of Applied Physics. 111, 10330211033028 (2012).

8 Editorial. 'Plenty of room' revisited. Nature Nanotechnology. 4, 781-781 (2009).

9 Novoselov, K. S. et al. Electric field effect in atomically thin carbon films. Science. 306, 666-669 (2004).

10 Iijima, S. Helical Microtubules of Graphitic Carbon. Nature. 354, 56-58 (1991).

11 Daniel, M. C. \& Astruc, D. Gold nanoparticles: assembly, supramolecular chemistry, quantum-size-related properties, and applications toward biology, catalysis, and nanotechnology. Chemical Reviews. 104, 293-346 (2004).

12 Quake, S. R. \& Scherer, A. From micro- to nanofabrication with soft materials. Science. 290, 1536-1540 (2000).

13 Salaita, K., Wang, Y. H. \& Mirkin, C. A. Applications of dip-pen nanolithography. Nature Nanotechnology. 2, 145-155 (2007).

14 Suzuki, H. Advances in the microfabrication of electrochemical sensors and systems. Electroanalysis. 12, 703-715 (2000).

15 Xia, S. \& Nihtianov, S. Power-efficient high-speed and high-resolution capacitive-sensor interface for subnanometer displacement measurements. IEEE Trans. Instrum. Meas. 61, 1315-1322 (2012).

16 Garcia, E. J. \& Sniegowski, J. J. Surface Micromachined Microengine. Sensors and Actuators A-Physical. 48, 203-214 (1995).

17 Trimmer, W. S. N. Microrobots and Micromechanical Systems. Sensors and Actuators. 19, 267-287 (1989).

18 Feynman, R. Infinitesimal machinery. J Microelectromech S. 2, 4-14 (1993). 
19 Chilkoti, A., Dreher, M. R., Meyer, D. E. \& Raucher, D. Targeted drug delivery by thermally responsive polymers. Advanced Drug Delivery Reviews. 54, 613-630 (2002).

20 Kopeček, J. Smart and genetically engineered biomaterials and drug delivery systems. European Journal of Pharmaceutical Sciences. 20, 1-16 (2003).

21 Sawant, R. M. et al. "SMART" drug delivery systems: double-targeted pHresponsive pharmaceutical nanocarriers. Bioconjugate Chemistry. 17, 943-949 (2006).

22 Marseille, O., Habib, N., Reul, H. \& Rau, G. Implantable micropump system for augmented liver perfusion. Artificial Organs. 22, 458-460 (1998).

23 Panchapakesan, B. et al. Micro- and nanotechnology approaches for capturing circulating tumor cells. Cancer Nano. 1, 3-11 (2010).

24 Binnig, G. \& Rohrer, H. Scanning tunneling microscopy. Helv Phys Acta. 55, 726-735 (1982).

25 Binnig, G., Quate, C. F. \& Gerber, C. Atomic force microscope. Phys Rev Lett. 56, 930-933 (1986).

26 Yoshida, S. Nanometrology. Metrologia. 28, 433-441 (1992).

27 Nakayama, K., Tanaka, M., Shiota, F. \& Kuroda, K. Precision physical measurements and nanometrology. Metrologia. 28, 483-502 (1992).

28 Feinberg, B. Dimensional metrology in manufacturing - need for relevance. Manuf Eng Manage. 70, 27-30 (1973).

29 Shao, Z. F., Yang, J. \& Somlyo, A. P. Biological atomic force microscopy: from microns to nanometers and beyond. Annu Rev Cell Dev Bi. 11, 241-265 (1995).

30 Brown, N. M. D. Scanning probe microscopies - applications in material science. Key Eng Mat. 99-1, 161-168 (1995).

31 Esch, J. Prolog to high-density data storage based on the atomic force microscope - an introduction to the paper by Mamin, Ried, Terris, and Rugar. P IEEE. 87, 1012-1013 (1999).

32 Mamin, H. J., Ried, R. P., Terris, B. D. \& Rugar, D. High-density data storage based on the atomic force microscope. P IEEE. 87, 1014-1027 (1999).

33 Elmustafa, A. A. \& Lagally, M. G. Flexural-hinge guided motion nanopositioner stage for precision machining: finite element simulations. Precis Eng. 25, 77-81 (2001).

34 Schumacher, H. W., Keyser, U. F., Zeitler, U., Haug, R. J. \& Eberl, K. Controlled mechanical AFM machining of two-dimensional electron systems: fabrication of a single-electron transistor. Physica E. 6, 860-863 (2000).

35 Yeh, H. C., Ni, W. T. \& Pan, S. S. Digital closed-loop nanopositioning using rectilinear flexure stage and laser interferometry. Control Eng Pract. 13, 559-566 (2005).

36 Shim, J. Y. \& Gweon, D. G. Piezo-driven metrological multiaxis nanopositioner. Rev Sci Instrum. 72, 4183-4187 (2001).

37 Uchino, K. et al. New monolithic actuators, monomorphs, using semiconductive ferroelectrics. Nippon Seram Kyo Gak. 95, 722-725 (1987).

38 Ganor, Y., Shilo, D., Messier, J., Shield, T. W. \& James, R. D. Testing system for ferromagnetic shape memory microactuators. Rev Sci Instrum. 78, 07390710739077 (2007). 
Uchino, K. Electrostrictive actuators - materials and applications. Am Ceram Soc Bull. 65, 647-652 (1986).

40 Hirai, T. et al. Polyurethane-elastomer-actuator. Angew Makromol Chem. 240, 221-229 (1996).

41 Pelrine, R., Kornbluh, R., Pei, Q. B. \& Joseph, J. High-speed electrically actuated elastomers with strain greater than 100\%. Science. 287, 836-839 (2000).

42 Lehmann, W. et al. Giant lateral electrostriction in ferroelectric liquid-crystalline elastomers. Nature. 410, 447-450 (2001).

43 Baughman, R. H. Conducting polymers in redox devices and intelligent materials systems. Makromol Chem-M Symp. 51, 193-215 (1991).

44 Baughman, R. H. Conducting polymer artificial muscles. Synthetic Metals. 78, 339-353 (1996).

45 Mirfakhrai, T., Madden, J. D. W. \& Baughman, R. H. Polymer artificial muscles. Materials Today. 10, 30-38 (2007).

46 Alici, G., Spinks, G., Huynh, N. N., Sarmadi, L. \& Minato, R. Establishment of a biomimetic device based on tri-layer polymer actuators - propulsion fins.

Bioinspiration \& Biomimetics. 2, S18 (2007).

47 Brochu, P. \& Pei, Q. B. Advances in dielectric elastomers for actuators and artificial muscles. Macromol. Rapid Commun. 31, 10-36 (2010).

48 Conway, N. J., Traina, Z. J. \& Kim, S. A strain amplifying piezoelectric MEMS actuator. Journal of Micromechanics and Microengineering. 17, 781 (2007).

49 Baughman, R. H. et al. Carbon nanotube actuators. Science. 284, 1340-1344 (1999).

50 Weissmuller, J. et al. Charge-induced reversible strain in a metal. Science. 300, 312-315 (2003).

51 Ahir, S. V. \& Terentjev, E. M. Photomechanical actuation in polymer-nanotube composites. Nature Materials. 4, 491-495 (2005).

52 Lu, S. X. \& Panchapakesan, B. Optically driven nanotube actuators. Nanotechnology. 16, 2548-2554 (2005).

53 Loomis, J., King, B. \& Panchapakesan, B. Layer dependent mechanical responses of graphene composites to near-infrared light. Applied Physics Letters. 100, 073108 (073104 pp.) (2012).

54 Loomis, J. et al. Graphene nanoplatelet based photomechanical actuators. Nanotechnology. 23, 045501-045510 (2012).

55 Loomis, J. \& Panchapakesan, B. Dimensional dependence of photomechanical response in carbon nanostructure composites: a case for carbon-based mixeddimensional systems. Nanotechnology. 23, 215501-215512 (2012).

56 Comstock, M. J. et al. Measuring reversible photomechanical switching rates for a molecule at a surface. Applied Physics Letters. 92, 1231071-1231073 (2008).

57 Lu, S. X. \& Panchapakesan, B. Nanotube micro-optomechanical actuators. Applied Physics Letters. 88, 253107 (2006).

58 Lu, S. X., Liu, Y., Shao, N. \& Panchapakesan, B. Nanotube micro-optomechanical systems. Nanotechnology. 18, 065501 (2007).

59 Uchino, K., Poosanaas, P. \& Tonooka, K. Photostrictive actuators - new perspective. Ferroelectrics. 264, 1961-1966 (2001). 
60 Yamada, M. et al. Photomobile polymer materials: towards light-driven plastic motors. Angew Chem Int Edit. 47, 4986-4988 (2008).

61 Lu, S. \& Panchapakesan, B. All-optical micromirrors from nanotube MOMS with wavelength selectivity. J Microelectromech S. 16, 1515-1523 (2007).

62 Ahir, S. V. \& Terentjev, E. M. Fast relaxation of carbon nanotubes in polymer composite actuators. Phys Rev Lett. 96, 133902 (2006).

63 Lu, S. X. \& Panchapakesan, B. Photomechanical responses of carbon nanotube/polymer actuators. Nanotechnology. 18, 305502 (2007).

64 Lu, S. X., Ahir, S. V., Terentjev, E. M. \& Panchapakesan, B. Alignment dependent mechanical responses of carbon nanotubes to light. Applied Physics Letters. 91, 103106 (2007).

65 Ahir, S. V., Terentjev, E. M., Lu, S. X. \& Panchapakesan, B. Thermal fluctuations, stress relaxation, and actuation in carbon nanotube networks. Phys Rev B. 76, 165437 (2007).

66 Balandin, A. A. et al. Superior thermal conductivity of single-layer graphene. Nano Lett. 8, 902-907 (2008).

67 Lu, Q., Arroyo, M. \& Huang, R. Elastic bending modulus of monolayer graphene. J Phys D Appl Phys. 42, 102002 (2009).

68 Gusynin, V. P. \& Sharapov, S. G. Unconventional integer quantum Hall effect in graphene. Phys Rev Lett. 95, 146801 (2005).

69 Sun, Z. P. et al. Graphene mode-locked ultrafast laser. Acs Nano. 4, 803-810 (2010).

70 Casiraghi, C. et al. Rayleigh imaging of graphene and graphene layers. Nano Lett. 7, 2711-2717 (2007).

71 Zhang, Y. B. et al. Direct observation of a widely tunable bandgap in bilayer graphene. Nature. 459, 820-823 (2009).

72 Nair, R. R. et al. Fine structure constant defines visual transparency of graphene. Science. 320, 1308-1308 (2008).

73 Obraztsov, P. A. et al. Broadband light-induced absorbance change in multilayer graphene. Nano Lett. 11, 1540-1545 (2011).

74 Lv, X. et al. Photoconductivity of bulk-film-based graphene sheets. Small. 5, 1682-1687 (2009).

75 Kuilla, T. et al. Recent advances in graphene based polymer composites. Prog Polym Sci. 35, 1350-1375 (2010).

76 Dreyer, D. R., Park, S., Bielawski, C. W. \& Ruoff, R. S. The chemistry of graphene oxide. Chem Soc Rev. 39, 228-240 (2010).

77 Gomez-Navarro, C., Burghard, M. \& Kern, K. Elastic properties of chemically derived single graphene sheets. Nano Lett. 8, 2045-2049 (2008).

78 Bielawski, C. W., Potts, J. R., Dreyer, D. R. \& Ruoff, R. S. Graphene-based polymer nanocomposites. Polymer. 52, 5-25 (2011).

79 Alexandre, M. \& Dubois, P. Polymer-layered silicate nanocomposites: preparation, properties and uses of a new class of materials. Mat Sci Eng R. 28, 163 (2000).

80 Thostenson, E. T., Li, C. Y. \& Chou, T. W. Nanocomposites in context. Compos Sci Technol. 65, 491-516 (2005). 
81 Lu, S. et al. Photo-mechanical actuation of carbon nanotubes: Mechanisms and applications in micro and nano-devices. Journal of Micro-Nano Mechatronics. 5, 29-41 (2009).

82 Curl, R. F. Fullerenes - collapse and growth. Nature. 363, 14-15 (1993).

83 Dresselhaus, M. S. et al. Raman spectroscopy of nanoscale carbons and of an isolated carbon nanotube. Mol Cryst Liq Cryst. 387, 245-253 (2002).

84 Kroto, H. W. Fullerene cage custers - the key to the structure of solid carbon. $J$ Chem Soc Faraday T. 86, 2465-2468 (1990).

85 Treolar, L. R. G. The physics of rubber elasticity. (Oxford University Press, 2005).

86 White, G. J. \& Padman, R. Images of atomic carbon in the interstellar medium. Nature. 354, 511-513 (1991).

87 Henning, T. \& Salama, F. Carbon in the universe. Science. 282, 2204-2210 (1998).

88 Ingber, D. E. The architecture of life. Scientific American. 278, 30-39 (1998).

89 Wehr, G., Sieber, G. \& Boning, K. Carbon resistors as low-temperature sensors in low-temperature reactor irradiation experiments. Cryogenics. 17, 43-45 (1977).

90 Thompson, S. P. \& Loughlan, J. Adaptive post-buckling response of carbon fibre composite plates employing SMA actuators. Compos Struct. 38, 667-678 (1997).

91 Hirsch, A. The era of carbon allotropes. Nature Materials. 9, 868-871 (2010).

92 King, R. J. Carbon, part 2: diamond. Geology Today. 24, 112-118 (2008).

93 Prasad, B., Rathod, S., Yadav, M. \& Modi, O. Sliding wear behavior of cast iron: influence of MoS and graphite addition to the oil lubricant. Journal of Materials Engineering and Performance. 20, 445-455 (2011).

94 Kroto, H. W., Heath, J. R., O'Brien, S. C., Curl, R. F. \& Smalley, R. E. C60: buckminsterfullerene. Nature. 318, 162-163 (1985).

95 Treacy, M. M. J., Ebbesen, T. W. \& Gibson, J. M. Exceptionally high Young's modulus observed for individual carbon nanotubes. Nature. 381, 678-680 (1996).

96 Bolotin, K. I. et al. Ultrahigh electron mobility in suspended graphene. Solid State Communications. 146, 351-355 (2008).

97 Novoselov, K. S. et al. Room-temperature quantum hall effect in graphene. Science. 315, 1379 (2007).

98 Banks, C. E., Davies, T. J., Wildgoose, G. G. \& Compton, R. G. Electrocatalysis at graphite and carbon nanotube modified electrodes: Edge-plane sites and tube ends are the reactive sites. Chemical Communications, 829-841 (2005).

99 Saito, R. et al. Optical absorption of graphite and single-wall carbon nanotubes. Applied Physics A: Materials Science \& Processing. 78, 1099-1105 (2004).

100 Faiman, D. et al. Structure and optical properties of C60 thin films. Thin Solid Films. 295, 283-286 (1997).

101 Pauling, L. The structure and properties of graphite and boron nitride. Proceedings of the National Academy of Sciences of the United States of America. 56, 1646-1652 (1966).

102 Lu, C. L., Chang, C. P., Huang, Y. C., Chen, R. B. \& Lin, M. L. Influence of an electric field on the optical properties of few-layer graphene with AB stacking. Physical Review B (Condensed Matter and Materials Physics). 73, 144427144421 (2006). 
103 Geim, A. K. \& Novoselov, K. S. The rise of graphene. Nature Materials. 6, 183191 (2007).

104 Heilmann, A. Polymer films with embedded metal nanoparticles. (Springer, 2003).

105 Chung, D. D. L. Review graphite. Journal of Materials Science. 37, 1475-1489 (2002).

106 Setton, R., Bernier, P. \& Lefrant, S. Carbon Molecules and Materials. (Taylor \& Francis, 2002).

107 Ergun, S. Structure of graphite. Nature physical science. 241, 65-67 (1973).

108 Gusynin, V. P. \& Sharapov, S. G. Transport of Dirac quasiparticles in graphene: Hall and optical conductivities. Physical Review B (Condensed Matter and Materials Physics). 73, 245411-245411 (2006).

109 Yacoby, A. Graphene tri and tri again. Nature Physics. 7, 925-926 (2011).

110 Dresselhaus, M. S., Dresselhaus, G. \& Avouris, P. Carbon Nanotubes Synthesis, Structure, Properties, and Applications. (Springer, 2001).

111 Xuekun, L., Minfeng, Y., Hui, H. \& Ruoff, R. S. Tailoring graphite with the goal of achieving single sheets. in 6th Foresight Conference, 12-15 Nov. 1998. 269272 (1999).

112 Inagaki, M. New Carbons: Control of Structure and Functions. (Elsevier, 2000).

113 Latil, S. \& Henrard, L. Charge carriers in few-layer graphene films. Phys Rev Lett. 97, 036803 (2006).

114 Nieto, A., Lahiri, D. \& Agarwal, A. Synthesis and properties of bulk graphene nanoplatelets consolidated by spark plasma sintering. Carbon. 50, 4068-4077 (2012).

115 Schultz, J., Lavielle, L. \& Martin, C. The role of the interface in carbon fibreepoxy composites. The Journal of Adhesion. 23, 45-60 (1987).

116 Pei, S. \& Cheng, H.-M. The reduction of graphene oxide. Carbon. 50, 3210-3228 (2012).

117 Miao, F., Hongbing, Z. \& Yu, C. Nonlinear optical and optical limiting properties of graphene families. Applied Physics Letters. 96, 0331071-0331073 (2010).

118 Mak, K. F. et al. Measurement of the optical conductivity of graphene. Phys Rev Lett. 101, 196405 (2008).

119 Guichuan, X., Hongchen, G., Xinhai, Z., Tze Chien, S. \& Huan, C. H. A. The physics of ultrafast saturable absorption in graphene. Optics Express. 18, 45644573 (2010).

120 Tabib-Azar, M. Microactuators: Electrical, Magnetic, Thermal, Optical, Mechanical, Chemical \& Smart Structures. (Springer, 1998).

121 Uchino, K. Piezoelectric actuators and ultrasonic motors. (Kluwer Academic Publishers, 1997).

122 El Feninat, F., Laroche, G., Fiset, M. \& Mantovani, D. Shape memory materials for biomedical applications. Advanced Engineering Materials. 4, 91-104 (2002).

123 Leff, H. S. Thermal efficiency at maximum work output: new results for old heat engines. American Journal of Physics. 55, 602-610 (1987).

124 Singh, M. Culture of heliotechnology. Renewable energy. 15, 224-229 (1998).

125 Sloley, R. W. Primitive methods of measuring time: with special reference to egypt. The Journal of Egyptian Archaeology. 17, 166-178 (1931). 
126 Chan, C. C. The rise \& fall of electric vehicles in 1828-1930: lessons learned. $P$ IEEE. 101, 206-212 (2013).

127 Miyahara, A. The live of Otto von Guericke and his achievements, available from Japanese Literature. Vakuum in Forschung und Praxis. 15, 316-322 (2003).

128 Kurkuri, M. D., Lee, J.-R., Han, J. H. \& Lee, I. Electroactive behavior of poly(acrylic acid) grafted poly(vinyl alcohol) samples, their synthesis using a Ce (IV) glucose redox system and their characterization. Smart Materials and Structures. 15, 417 (2006).

129 Tzou, H. S., Lee, H. J. \& Arnold, S. M. Smart materials, precision sensors/actuators, smart structures, and structronic systems. Mechanics of Advanced Materials and Structures. 11, 367-393 (2004).

130 Rao, J. S. History of rotating machinery dynamics. (Springer, 2011).

131 Curie, P. \& Curie, J. Development by pressure of polar electricity in hemihedral crystals with inclined faces. Bull. Soc. Min. de France. 3, 90 (1880).

132 Ölander, A. \& Kristallogr, Z. The crystal structure of AuCd. Zeitschrift Fur Kristallographie. 83A, 145-148 (1932).

133 Sharapov, V. M. Piezoceramic sensors. (SpringerLink, 2011).

134 Zelenka, J. Piezoelectric resonators and their applications. (Elsevier, 1986).

135 Piezoelectric Actuators and Motors - Types, Applications, New Developments, Industry Structure and Global Markets. 154 (Innovative Research and Products (iRAP), Inc. , 2010).

136 Pulskamp, J. S. et al. Piezoelectric PZT MEMS technologies for small-scale robotics and RF applications. MRS Bull. 37, 1062-1070 (2012).

137 Korayem, M. H. \& Omidi, E. Robust controlled manipulation of nanoparticles using atomic force microscope. Micro Nano Lett. 7, 927-931 (2012).

138 Sherlock, N. P., Garten, L. M., Zhang, S. J., Shrout, T. R. \& Meyer, R. J. Nonlinear dielectric response in piezoelectric materials for underwater transducers. Journal of Applied Physics. 112, 124108 (2012).

139 Kwak, J., Kingon, A. I. \& Kim, S. H. Lead-free (Na-0.5,K-0.5) $\mathrm{NbO}_{3}$ thin films for the implantable piezoelectric medical sensor applications. Mater. Lett. 82, 130-132 (2012).

140 Mancini, G., Buonaccorsi, S., Reale, G. \& Tedaldi, M. Application of piezoelectric device in endoscopic sinus surgery. J. Craniofac. Surg. 23, 17361740 (2012).

141 Palm, L., Wallman, L., Laurell, T. \& Nilsson, J. Development and characterization of silicon micromachined nozzle units for continuous ink jet printers. J. Imaging Sci. Technol. 44, 544-551 (2000).

142 Wang, X. D., Song, J. H., Liu, J. \& Wang, Z. L. Direct-current nanogenerator driven by ultrasonic waves. Science. 316, 102-105 (2007).

143 DeVoe, D. L. \& Pisano, A. P. Surface micromachined piezoelectric accelerometers (PiXLs). J Microelectromech S. 10, 180-186 (2001).

144 Janocha, H. Adaptronics and Smart Structures. (Springer, 2007).

145 A. V. Srinivasan, D. M. M. Smart Structures, Analysis and Design. (Cambridge University Press, 2001).

146 K. Worden, W. A. B., J. Haywood. Smart Technologies. (World Scientific Publishing, 2003). 
147 Nishimura, K. A spring-guided micropositioner with linearized subnanometer resolution. Rev Sci Instrum. 62, 2004-2007 (1991).

148 Roseman, M. \& Grutter, P. Cryogenic magnetic force microscope. Rev Sci Instrum. 71, 3782-3787 (2000).

149 Hall, D. A. Review nonlinearity in piezoelectric ceramics. Journal of Materials Science. 36, 4575-4601 (2001).

150 Wei, Z. G., Sandstroröm, R. \& Miyazaki, S. Shape-memory materials and hybrid composites for smart systems: Part I shape-memory materials. Journal of Materials Science. 33, 3743-3762 (1998).

151 Buehler, W. J., Gilfrich, J. V. \& Weiley, K. C. Effect of low-temperature phase changes on the mechanical properties of alloys near composition TiNi. J. Appl. Phys. 34, 1475-1477 (1963).

152 Bogue, R. Shape-memory materials: A review of technology and applications. Assembly Automation. 29, 214-219 (2009).

153 Garner, L. J., Wilson, L. N., Lagoudas, D. C. \& Rediniotis, O. K. Development of a shape memory alloy actuated biomimetic vehicle. Smart Materials and Structures. 9, 673-683 (2000).

154 Thrasher, M. A., Shahin, A. R., Meckl, P. H. \& Jones, J. D. Efficiency analysis of shape memory alloy actuators. Smart Materials and Structures. 3, 226-234 (1994).

155 Ekreem, N. B., Olabi, A. G., Prescott, T., Rafferty, A. \& Hashmi, M. S. J. An overview of magnetostriction, its use and methods to measure these properties. Journal of Materials Processing Technology. 191, 96-101 (2007).

156 Bar-Cohen, Y. Electroactive polymer (EAP) actuators as artificial muscles. (SPIE Press, 2004).

157 Engdahl, G. Handbook of giant magnetostrictive materials (Academic Press 1999).

158 Quandt, E. \& Ludwig, A. Magnetostrictive actuation in microsystems. Sensors and Actuators A: Physical. 81, 275-280 (2000).

159 Hugel, T. et al. Single-molecule optomechanical cycle. Science. 296, 1103-1106 (2002).

160 Knopf, G. K. \& Otani, Y. Optical nano and micro actuator technology. (CRC Press, 2013).

161 Stuchlik, M., Krecmer, P. \& Elliott, S. R. Opto-mechanical effect in chalcogenide glasses. Journal of Optoelectronics and Advanced Materials. 3, 361-366 (2001).

162 Spearing, S. M. Materials issues in microelectromechanical systems (MEMS). Acta Materialia. 48, 179-196 (2000).

163 Tanaka, K., Saitoh, A. \& Terakado, N. Giant photo-expansion in chalcogenide glass. Journal of Optoelectronics and Advanced Materials. 8, 2058-2065 (2006).

164 Popescu, M. Chalcogenides - past, present, future. Journal of Non-Crystalline Solids. 352, 887-891 (2006).

165 Camacho-Lopez, M., Finkelmann, H., Palffy-Muhoray, P. \& Shelley, M. Fast liquid-crystal elastomer swims into the dark. Nature Materials. 3, 307-310 (2004).

166 Koerner, H., Price, G., Pearce, N. A., Alexander, M. \& Vaia, R. A. Remotely actuated polymer nanocomposites - stress-recovery of carbon-nanotube-filled thermoplastic elastomers. Nature Materials. 3, 115-120 (2004). 
167 Datskos, P. G., Rajic, S. \& Datskou, I. Photoinduced and thermal stress in silicon microcantilevers. Applied Physics Letters. 73, 2319-2321 (1998).

168 Sulfridge, M., Saif, T., Miller, N. \& O"Hara, K. Optical actuation of a bistable MEMS. J Microelectromech S. 11, 574-583 (2002).

169 Lendlein, A., Hongyan, J., Junger, O. \& Langer, R. Light-induced shape-memory polymers. Nature. 434, 879-882 (2005).

170 Schuerman, D. W. The restricted three-body problem including radiation pressure. Astrophysical Journal. 238, 337-342 (1980).

171 Tsu, T. C. Solar energy; solar radiation; radiation pressure; space vehicles; propulsion; orientation; radiations. $A R S$ - J. 29, 422-427 (1959).

172 Anderson, J. D. et al. Indication, from Pioneer 10/11, Galileo, and Ulysses data, of an apparent anomalous, weak, long-range acceleration. Phys Rev Lett. 81, 2858-2861 (1998).

173 Springer, T. A., Beutler, G. \& Rothacher, M. A new solar radiation pressure model for GPS satellites. GPS Solutions. 2, 50-62 (1999).

174 Dragoman, D. \& Dragoman, M. Optical actuation of micromechanical tunneling structures with applications in spectrum analysis and optical computing. Appl. Opt. 38, 6773-6778 (1999).

175 Yang, J., Ono, T. \& Esashi, M. Surface effects and high quality factors in ultrathin single-crystal silicon cantilevers. Applied Physics Letters. 77, 3860-3862 (2000).

176 Karastojkovic, Z. et al. High reflectance as desired property of shining surface and undesired for laser welding in jewel production of 585 gold alloy. Metal. Int. 18, 39-42 (2013).

177 Oliver, A. D., Vigil, S. R. \& Gianchandani, Y. B. Photothermal surfacemicromachined actuators. Electron Devices, IEEE Transactions on. 50, 11561157 (2003).

178 Elbuken, C., Gui, L., Ren, C. L., Yavuz, M. \& Khamesee, M. B. Design and analysis of a polymeric photo-thermal microactuator. Sensors and Actuators A: Physical. 147, 292-299 (2008).

179 Bergstrom, P. L., Jin, J., Yu-Ning, L., Kaviany, M. \& Wise, K. D. Thermally driven phase-change microactuation. J Microelectromech S. 4, 10-17 (1995).

180 Otani, Y., Matsuba, Y.\& Osaka, A. Microbubble actuator using photothermal effect. in Optomechatronic Systems III, 12-14 Nov. 2002. 83-86 (2002).

181 Que, L., Park, J. S. \& Gianchandani, Y. B. Bent-beam electro-thermal actuators for high force applications. in Twelfth IEEE International Conference on MEMS. 31-36 (1999).

182 Liwei, L., Pisano, A. P. \& Lee, A. P. Microbubble powered actuator. in Digest of Technical Papers, Transducers 1991. 1041-1044 (1991).

183 Zakery, A. \& Elliott, S. R. Optical properties and applications of chalcogenide glasses: a review. Journal of Non-Crystalline Solids. 330, 1-12 (2003).

184 Stuchlik, M. \& Elliott, S. R. All-optical actuation of amorphous chalcogenidecoated cantilevers. Journal of Non-Crystalline Solids. 353, 250-262 (2007).

185 Hisakuni, H. \& Tanaka, K. Giant photoexpansion in $\mathrm{As}_{2} \mathrm{~S}_{3}$ glass. Applied Physics Letters. 65, 2925-2927 (1994). 
186 Ganjoo, A., Jain, H. \& Khalid, S. Atomistic observation of photo-expansion and photo-contraction in chalcogenide films by in situ EXAFS. Journal of NonCrystalline Solids. 354, 2673-2678 (2008).

187 Ohm, C., Brehmer, M. \& Zentel, R. Liquid crystalline elastomers as actuators and sensors. Advanced Materials. 22, 3366-3387 (2010).

188 Woltman, S. J., Jay, G. D. \& Crawford, G. P. Liquid-crystal materials find a new order in biomedical applications. Nature Materials. 6, 929-938 (2007).

189 Alici, G., Mui, B. \& Cook, C. Bending modeling and its experimental verification for conducting polymer actuators dedicated to manipulation applications. Sensors and Actuators A: Physical. 126, 396-404 (2006).

190 Cai, D. K., Neyer, A., Kuckuk, R. \& Heise, H. M. Optical absorption in transparent PDMS materials applied for multimode waveguides fabrication. Optical Materials. 30, 1157-1161 (2008).

191 Rogers, G. W. \& Liu, J. Z. Graphene actuators: quantum-mechanical and electrostatic double-layer effects. J Am Chem Soc. 133, 10858-10863 (2011).

192 Armani, D., Liu, C. \& Aluru, N. Re-configurable fluid circuits by PDMS elastomer micromachining. in Twelfth IEEE International Conference on MEMS. (1999).

193 Sarkisov, S. S., Curley, M. J., Fields, A., Sarkisov, S. S. \& Adamovsky, G. Photomechanical effect in films of polyvinylidene fluoride. Applied Physics Letters. 85, 2747-2749 (2004).

194 Seki, T. \& Tamaki, T. Photomechanical effect in monolayers of azobenzene sidechain polymers. Chem Lett, 1739-1742 (1993).

195 Tabiryan, N., Serak, S., Dai, X. M. \& Bunning, T. Polymer film with optically controlled form and actuation. Optics Express. 13, 7442-7448 (2005).

196 Ikeda, T., Mamiya, J. \& Yu, Y. L. Photomechanics of liquid-crystalline elastomers and other polymers. Angew Chem Int Edit. 46, 506-528 (2007).

197 Thomsen, D. L. et al. Liquid crystal elastomers with mechanical properties of a muscle. Macromolecules. 34, 5868-5875 (2001).

198 Ferrari, A. C. Raman spectroscopy of graphene and graphite: disorder, electronphonon coupling, doping and nonadiabatic effects. Solid State Communications. 143, 47-57 (2007).

199 Sarkisov, S. S., Curley, M. J., Huey, L., Fields, A. \& Adamovsky, G. Light-driven actuators based on polymer films. Optical Engineering. 45, 34302 (2006).

200 Dresselhaus, M. S., Dresselhaus, G. \& Hofmann, M. Raman spectroscopy as a probe of graphene and carbon nanotubes. Philosophical Transactions of the Royal Society A: Mathematical, Physical and Engineering Sciences. 366, 231-236 (2008).

201 Mark, J. E. Some recent theory, experiments, and simulations on rubberlike elasticity. The Journal of Physical Chemistry B. 107, 903-913 (2002).

202 Stafford, C. M., Shu, G., Harrison, C. \& Chiang, M. Y. M. Combinatorial and high-throughput measurements of the modulus of thin polymer films. Rev Sci Instrum. 76, 62207 (2005).

203 Fuard, D., Tzvetkova-Chevolleau, T., Decossas, S., Tracqui, P. \& Schiavone, P. Optimization of poly-di-methyl-siloxane (PDMS) substrates for studying cellular adhesion and motility. Microelectronic Engineering. 85, 1289-1293 (2008). 
204 Coleman, J. N. et al. Percolation-dominated conductivity in a conjugatedpolymer-carbon-nanotube composite. Phys Rev B. 58, R7492-R7495 (1998).

205 Williams, G. \& Watts, D. C. Non-symmetrical dielectric relaxation behaviour arising from a simple empirical decay function. Transactions of the Faraday Society. 66, 80-85 (1970).

206 Balberg, I., Binenbaum, N. \& Wagner, N. Percolation thresholds in the threedimensional sticks system. Phys Rev Lett. 52, 1465-1468 (1984).

207 Badaire, S., Poulin, P., Maugey, M. \& Zakri, C. In situ measurements of nanotube dimensions in suspensions by depolarized dynamic light scattering. Langmuir. 20, 10367-10370 (2004).

208 Bauhofer, W. \& Kovacs, J. Z. A review and analysis of electrical percolation in carbon nanotube polymer composites. Compos Sci Technol. 69, 1486-1498 (2009).

209 Sandler, J. K. W., Kirk, J. E., Kinloch, I. A., Shaffer, M. S. P. \& Windle, A. H. Ultra-low electrical percolation threshold in carbon-nanotube-epoxy composites. Polymer. 44, 5893-5899 (2003).

210 Zhang, Q., Rastogi, S., Chen, D., Lippits, D. \& Lemstra, P. J. Low percolation threshold in single-walled carbon nanotube/high density polyethylene composites prepared by melt processing technique. Carbon. 44, 778-785 (2006).

211 Lyons, P. E. et al. The relationship between network morphology and conductivity in nanotube films. Journal of Applied Physics. 104, 044302 (2008).

212 Lu, S. \& Panchapakesan, B. Photoconductivity in single wall carbon nanotube sheets. Nanotechnology. 17, 1843-1850 (2006).

213 Zuev, Y. M., Chang, W. \& Kim, P. Thermoelectric and magnetothermoelectric transport measurements of graphene. Phys Rev Lett. 102, 096807 (2009).

214 Shi, L., Yu, C. H., Yao, Z., Li, D. Y. \& Majumdar, A. Thermal conductance and thermopower of an individual single-wall carbon nanotube. Nano Lett. 5, 18421846 (2005).

215 Xu, X. D., Gabor, N. M., Alden, J. S., van der Zande, A. M. \& McEuen, P. L. Photo-thermoelectric effect at a graphene interface junction. Nano Lett. 10, 562566 (2010).

$216 \mathrm{Yu}$, A. P. et al. Enhanced thermal conductivity in a hybrid graphite nanoplatelet carbon nanotube filler for epoxy composites. Advanced Materials. 20, 4740-+ (2008).

217 Ajayan, P. M., Stephan, O., Colliex, C. \& Trauth, D. Aligned carbon nanotube arrays formed by cutting a polymer resin-nanotube composite. Science. 265, 1212-1214 (1994).

218 Suhr, J., Koratkar, N., Keblinski, P. \& Ajayan, P. Viscoelasticity in carbon nanotube composites. Nature Materials. 4, 134-137 (2005).

219 Miaudet, P. et al. Shape and temperature memory of nanocomposites with broadened glass transition. Science. 318, 1294-1296 (2007).

220 Smith, J. G. et al. Space durable polymer/carbon nanotube films for electrostatic charge mitigation. Polymer. 45, 825-836 (2004).

221 Kashiwagi, T. et al. Nanoparticle networks reduce the flammability of polymer nanocomposites. Nature Materials. 4, 928-933 (2005). 
222 Chun, K. Y. et al. Highly conductive, printable and stretchable composite films of carbon nanotubes and silver. Nature Nanotechnology. 5, 853-857 (2010).

223 Fukushima, T. et al. Molecular ordering of organic molten salts triggered by single-walled carbon nanotubes. Science. 300, 2072-2074 (2003).

224 Peng, H. S. et al. Electrochromatic carbon nanotube/polydiacetylene nanocomposite fibres. Nature Nanotechnology. 4, 738-741 (2009).

225 Lipomi, D. J. et al. Skin-like pressure and strain sensors based on transparent elastic films of carbon nanotubes. Nature Nanotechnology. 6, 788-792 (2011).

226 Ajayan, P. M. \& Tour, J. M. Materials science - Nanotube composites. Nature. 447, 1066-1068 (2007).

227 Madden, J. D. Mobile robots: Motor challenges and materials solutions. Science. 318, 1094-1097 (2007).

228 Samel, B., Griss, P. \& Stemme, G. Expandable microspheres incorporated in a PDMS matrix: a novel thermal composite actuator for liquid handling in microfluidic applications. in IEEE International Solid-State Sensors and Actuators. 1558-1561 (2003).

229 Tsagaropoulos, G. \& Eisenburg, A. Direct observation of two glass transitions in silica-filled polymers. Implications to the morphology of random ionomers. Macromolecules. 28, 396-398 (1995).

230 Fröhlich, J., Niedermeier, W. \& Luginsland, H. D. The effect of filler-filler and filler-elastomer interaction on rubber reinforcement. Composites Part A: Applied Science and Manufacturing. 36, 449-460 (2005).

231 Berriot, J., Montes, H., Lequeux, F., Long, D. \& Sotta, P. Gradient of glass transition temperature in filled elastomers. Europhysics Letters. 64, 50-56 (2003).

232 Fritzsche, J. \& Kluppel, M. Structural dynamics and interfacial properties of filler-reinforced elastomers. Journal of Physics: Condensed Matter. 23, 1-11 (2011).

233 Merabia, S., Sotta, P. \& Long, D. R. Unique plastic and recovery behavior of nanofilled elastomers and thermoplastic elastomers (Payne and Mullins effects). Journal of Polymer Science, Part B: Polymer Physics. 48, 1495-1508 (2010).

234 Zhang, W. D., Shen, L., Phang, I. Y. \& Liu, T. Carbon nanotubes reinforced nylon-6 composite prepared by simple melt-compounding. Macromolecules. 37, 256-259 (2004).

235 Coleman, J. N., Khan, U., Blau, W. J. \& Gun'ko, Y. K. Small but strong: A review of the mechanical properties of carbon nanotube-polymer composites. Carbon. 44, 1624-1652 (2006).

236 Ci, L., Suhr, J., Pushparaj, V., Zhang, X. \& Ajayan, P. M. Continuous carbon nanotube reinforced composites. Nano Lett. 8, 2762-2766 (2008).

237 Barone, P. W., Baik, S., Heller, D. A. \& Strano, M. S. Near-infrared optical sensors based on single-walled carbon nanotubes. Nature Materials. 4, 86-92 (2005).

238 Boller, C. Adaptronic systems for aerospace applications. Automatisierungstechnik. 54, 276-283 (2006).

239 Buter, A. \& Breitbach, E. Adaptive blade twist - Calculations and experimental results. Aerospace Science and Technology. 4, 309-319 (2000). 
240 Durr, J. K., Honke, R., von Alberti, M. \& Sippel, R. Development and manufacture of an adaptive lightweight mirror for space application. Smart Materials and Structures. 12, 1005-1016 (2003).

241 Glurgiutiu, V., Bayouml, A. M. E. \& Nall, G. Mechatronics and smart structures: emerging engineering disciplines for the third millennium. Mechatronics. 12, 169181 (2002).

242 Ast, A., Braun, S., Eberhard, P. \& Heisel, U. An adaptronic approach to active vibration control of machine tools with parallel kinematics. Production Engineering. 3, 207-215 (2009).

243 Van Lintel, H. T. G., Van De Pol, F. C. M. \& Bouwstra, S. A piezoelectric micropump based on micromachining of silicon. Sensors and Actuators. 15, 153167 (1988).

244 Bromberg, L. E. \& Ron, E. S. Temperature-responsive gels and thermogelling polymer matrices for protein and peptide delivery. Advanced Drug Delivery Reviews. 31, 197-221 (1998).

245 Balandin, A. A. Thermal properties of graphene and nanostructured carbon materials. Nature Materials. 10, 569-581 (2011).

$246 \mathrm{Wu}, \mathrm{H}$. \& Drzal, L. T. Graphene nanoplatelet paper as a light-weight composite with excellent electrical and thermal conductivity and good gas barrier properties. Carbon. 50, 1135-1145 (2012).

247 Mark, J. E. \& Erman, B. Rubberlike elasticity, a molecular primer. (Cambridge University Press, 2007).

248 Choi, S. U. S., Zhang, Z. G., Yu, W., Lockwood, F. E. \& Grulke, E. A. Anomalous thermal conductivity enhancement in nanotube suspensions. Applied Physics Letters. 79, 2252-2254 (2001).

249 Gabor, N. M. et al. Hot carrier-assisted intrinsic photoresponse in graphene. Science. 334, 648-652 (2011).

250 Hubbard, N. B. \& Howell, L. L. Design and characterization of a dual-stage, thermally actuated nanopositioner. Journal of Micromechanics and Microengineering. 15, 1482-1493 (2005).

251 Zhu, Y., Bazaei, A., Moheimani, S. O. R. \& Yuce, M. R. A micromachined nanopositioner with on-chip electrothermal actuation and sensing. IEEE Electr Device L. 31, 1161-1163 (2010).

252 Bokobza, L. The reinforcement of elastomeric networks by fillers. Macromolecular Materials and Engineering. 289, 607-621 (2004).

253 Soni, V. K. \& Stein, R. S. Light scattering studies of poly(dimethylsiloxane) solutions and swollen networks. Macromolecules. 23, 5257-5265 (1990). 
APPENDICES

APPENDIX A

\section{SAMPLE PREPARATION OF GNP/PDMS COMPOSITES:}

PDMS silicone elastomer obtained from Dow Corning (Sylgard 184) was used as the host matrix. PDMS is a two-part solvent-free flexible silicone organic polymer in the form of a base compound with a separate hydrosilane curing agent that acts as a crosslinker. The term "wt\%" refers to the ratio of carbon additive to PDMS base compound. PDMS composites were fabricated by weighing the desired amount of additive (GNPs, CNTs, GO, or CB) and adding to the PDMS base compound. The additive/base compound combination was then shear mixed for 5 min to facilitate carbon distribution. Taking into account the additive weight, a crosslinker was added at a ratio of 1:10 and further shear mixed for $5 \mathrm{~min}$. To remove trapped air pockets, prepared polymers were degassed for $30 \mathrm{~min}$. Small amounts of liquid polymer mixtures were deposited on the glass slides. A standard spin coating process at $750 \mathrm{rpm}$ for $150 \mathrm{~s}$ successfully produced nominal $60 \mu \mathrm{m}$ thick films. High temperature curing at $125^{\circ} \mathrm{C}$ for 20 min was employed to finish the cross-linking process in the polymer. PDMS composite sample dimensions were approximately $60 \times 3 \mathrm{~mm}(50 \mathrm{~mm}$ of active test area, with $5 \mathrm{~mm}$ on each side for test fixture mounting). 


\section{STRESS TEST EXPERIMENTATION:}

PDMS composite test samples were mounted vertically between two clamps [Figure 3.1(c) shows this setup]. The bottom clamp was attached to a weighted (50 g) base and placed on a high accuracy balance (Acculab ALC-80.4). The upper clamp was attached to an automated linear actuator that was in turn mounted to a high accuracy manual positioning stage. The laser diode was placed $\sim 75 \mathrm{~mm}$ from the middle of the test strip such that illumination impacted normal to the PDMS surface. Deformation in the composite strips as a result of NIR illumination caused a change in weight readings on the balance. Once the light was turned off, both the actuator and balance returned to their original length/reading, respectively. Actuation was quite repeatable from cycle to cycle with nearly the same displacement amplitude. Stress test standardization was accomplished by finding the zero strain value of each sample and zeroing the balance. Stress tests on each sample were conducted with pre-strain values ranging from $3 \%$ to $40 \%$. The timing sequence for each pre-strain value was 1 -min relaxation wait followed by 5 cycles of NIR illumination on for $60 \mathrm{~s}$, and then off for $30 \mathrm{~s}$. Engineering stress calculations (referred to as stress throughout the paper) were made by dividing the change in force between illumination on and off by the cross-sectional area of the test samples.

\section{YOUNG'S MODULUS CALCULATIONS:}

Young's modulus values as a function of increasing wt $\%$ GNP/PDMS were determined by first measuring the stress induced by pre-straining each test sample from 0 to $5 \%$ in $1 \%$ intervals (NIR illumination $o f f$ ). For a given $w t \%$ GNP/PDMS, an average

of the resulting change in stress divided by the pre-strain (for pre-strains from $0-5 \%$ ) 
yielded values for the elastic modulus. GNP/PDMS test samples were observed to have an elastic behavior in this region.

OPTICAL-TO-MECHANICAL ENERGY CONVERSION CALCULATION:

$$
\begin{aligned}
& P_{\text {effective }}=\left(P_{\text {laser }} / A_{\text {spot }}\right) * A_{\text {incident }} \\
& \eta_{\mathrm{M}}=\Delta \sigma_{\text {total }} / P_{\text {effective }}
\end{aligned}
$$

A laser power meter (Newport 1918-C) was used to calculate laser power absorbed in the test sample. First, an uninterrupted laser power of $\sim 500 \mathrm{~mW}$ was measured. Next, a $2 \mathrm{wt} \% \mathrm{GNP} / \mathrm{PDMS}$ test sample was mounted in front of the meter and a value of $\sim 160 \mathrm{~mW}$ obtained. Therefore, the difference $(\sim 340 \mathrm{~mW})$ is the amount of power absorbed in the test sample as referred to as $\left(P_{\text {laser }}\right)$ throughout. $A_{\text {spot }}$ is the area of the spot size $\left(3 \times 3 \mathrm{~mm}\right.$, or $\left.9 \mathrm{~mm}^{2}\right)$, and $A_{\text {incident }}$ is the area for absorption within the film ( $3 \mathrm{~mm} \times 60 \mu \mathrm{m}$, or $0.18 \mathrm{~mm}^{2}$ ). The effective laser power [equation (A1), $P_{\text {effective], }}$, therefore, is $6.8 \mathrm{~mW}$. The optical-to-mechanical energy conversion factor [equation (A2), $\left.\eta_{\mathrm{M}}\right]$ is calculated by dividing the total change in engineering stress ( $\left.\Delta \sigma_{\text {total }}\right)$ from maximum expansion to maximum contraction $(+14 \mathrm{kPa}$ to $-36 \mathrm{kPa}$, or $50 \mathrm{kPa})$ by the $P_{\text {effective, }}$ resulting in a value of $7-9 \mathrm{MPa} / \mathrm{W}$.

\section{THERMAL RESPONSE TESTING:}

Steady-state temperature tests were conducted by placing a thermocouple (Omega, thin wire K-type) in contact with the PDMS composite. Temperature measurements were conducted in $5 \mathrm{~mm}$ intervals from the illumination point to the edge of the testing area ( $25 \mathrm{~mm}$ for $0 \%$ pre-strain, $35 \mathrm{~mm}$ for $40 \%$ pre-strain). Each position 
was held for 5 min to allow temperature to reach steady-state value (typically reached within $2 \mathrm{~min}$ ). Plotted temperature data points were calculated by averaging the last $60 \mathrm{~s}$ of each test period. 


\section{APPENDIX B}

\section{SAMPLE PREPARATION OF SLG, CNT, GNP AND HOPG-BASED COMPOSITES:}

$150 \mathrm{mg}$ of carbon additive (SLG, MWNT, GNP, or HOPG) was mixed with 100 $\mathrm{mL}$ of IPA and sonicated for $4 \mathrm{~h}$ at $50^{\circ} \mathrm{C}$ (Cole-Palmer 8892). After sonication, $15 \mathrm{~g}$ of PDMS base compound was added to the carbon/IPA mixture. This solution was placed on a $100^{\circ} \mathrm{C}$ hotplate and a magnetic stir bar added. During $\sim 18 \mathrm{~h}$, the IPA slowly evaporated leaving carbon/PDMS base compound mixture. Temperature was monitored such that the solution never boiled, but rather was kept warm. The carbon was easily dispersed in the IPA, and the homogeneous dispersion transferred to the PDMS base compound. The carbon/PDMS base compound changed consistency when the IPA was boiled off. Removal of the IPA was verified through consistency as well as container markings indicating initial PDMS base compound level. After removal of the IPA, the $1 \mathrm{wt} \%$ evaporative mixed carbon additive/PDMS base compound solution was diluted with pristine PDMS base compound to $\mathrm{wt} \%$ ratios of $0.5,0.1$, and 0.01 . Taking into account the carbon additive, crosslinker was then added at a ratio of 1:10 and shear mixed for $5 \mathrm{~min}$. To remove trapped air pockets, prepared polymers were degassed for $30 \mathrm{~min}$. Small amounts $(\sim 3 \mathrm{~g})$ of liquid polymer mixtures were deposited on the glass slides. A spin coating process at $750 \mathrm{rpm}$ for $150 \mathrm{~s}$ produced nominal $60 \mu \mathrm{m}$ thick films. Curing at $125^{\circ} \mathrm{C}$ for $20 \mathrm{~min}$ was employed to finish the cross-linking process in the polymer, and samples were left for a minimum of $12 \mathrm{~h}$ at room temperature prior to testing. 


\section{APPENDIX C}

\section{LASER POWER MEASUREMENT:}

A laser power meter (Newport 1918-C) measured laser power transmitted through the sample. Laser spot size was $\sim 3 \mathrm{~mm} \times 3 \mathrm{~mm}$, therefore area exposed to NIR radiation was $\sim 9 \mathrm{~mm}^{2}$. Power absorbed in the samples was determined by subtracting power meter readings during sample testing from total laser power.

\section{STRESS TEST EXPERIMENTATION:}

Photo-mechanical stress response to NIR illumination as a function of pre-strain was evaluated for pre-strains ( $(\varepsilon)$ ranging from 3-40\%. At each pre-strain value, test composites underwent 5 cycles of NIR illumination on for $60 \mathrm{~s}$, followed by NIR illumination off for $30 \mathrm{~s}$. Magnitude of actuation was highly repeatable for each test sample, and composites exhibited elastic response characteristics throughout all pre-strain values. PDMS composite test samples were mounted vertically between two clamps. The bottom clamp was attached to a weighted (65 g) base and placed on a high accuracy balance (Acculab ALC-80.4). The upper clamp was attached to an automated linear actuator that was in turn mounted to a high accuracy manual positioning stage. The NIR laser source was mounted on a linear actuator with the diode placed $\sim 200 \mathrm{~mm}$ from the center of the test strip such that illumination impacted normal to the PDMS surface. Electrodes were attached to each end of the test sample and resistance monitored on a 
Keithley 2700 digital multimeter. Deformation in composite strips as a result of NIR illumination caused a change in weight readings on the balance. Once light was turned off, both the actuator and balance returned to their original length/reading, respectively. Actuation was quite repeatable from cycle to cycle with nearly the same displacement amplitude. Stress test standardization was accomplished by finding the zero strain value of each sample and zeroing the balance. Engineering stress calculations (referred to as stress throughout the dissertation) were made by dividing the change in force between illumination on and off by the cross-sectional area of the test samples. Long-term stress responses consisted of 500 NIR on/off cycles conducted at 3\% and $20 \%$ pre-strains, for a total of 1,000 cycles over $\sim 24 \mathrm{~h}$. For clarification and comparison, NIR off stresses were "zeroed", resulting in relative NIR-induced stress graphs as shown in Figure 4(a) (right side).

\section{EFFICIENCY CALCULATIONS:}

Efficiency values were calculated at every pre-strain value for each carbon nanostructure type and concentration using the spring model. For pre-strains of up to $40 \%$, force was found to be approximately linearly proportional to the displacement; therefore justifying use of the spring model. First, force photo-mechanical forces $(F)$ as a function of NIR-induced stress $(\sigma)$ and composite cross sectional areas ( $A$, typically about $0.18 \mathrm{~mm}^{2}$ ) were determined [equation (C1)].

$$
F=\sigma A
$$

Hook's law was rearranged in order to determine spring constants for the elastic samples [equation (C2), where $x$ is NIR-induced photo-mechanical deformation]. 


$$
k=-F / x
$$

Next, the spring constant was used to find potential energies [equation (C3)] at each prestrain were determined, and used to calculate actuator efficiencies $(\eta)$ using equation (C4).

$$
\begin{aligned}
& P E=k x^{2} / 2 \\
& \eta=P E /(P \tau)
\end{aligned}
$$

Supplied laser energies [equation (C6), $P$ ] were determined by multiplying the power absorbed by the sample $\left(P_{\text {absorbed }}\right)$ by the incident area through the sample [equation $(\mathrm{C} 5)$, $\left.A_{\text {incident }}\right]$ and dividing by the NIR illumination point size $\left(A_{\text {point }}\right)$.

$$
\begin{aligned}
& A_{\text {incident }}=h_{N I R} \times t_{\text {sample }} \\
& P=P_{\text {absorbed }} \times A_{\text {incident }} / A_{\text {point }}
\end{aligned}
$$

Actuation time constant $(\tau)$ values were found to vary between $\sim 5 \mathrm{~s}$ to $\sim 10 \mathrm{~s}$. Therefore, a standard value of $10 \mathrm{~s}$ was used in order to obtain the most conservative results. 
APPENDIX D

\section{CNT/EXPANDING MICROSPHERE/POLYMER COMPOSITE FABRICATION:}

A $1 \mathrm{mg} / \mathrm{mL}$ concentration $\mathrm{SWNT} /$ isopropanol (IPA) solution was sonicated for $\sim 4$ h. Separately, PDMS base compound was heated to $160^{\circ} \mathrm{C}$, while a magnetic stir bar (60 rpm) provided agitation. Approximately $\sim 1 \mathrm{~mL} / \mathrm{min}$ of SWNT/IPA solution was added to the PDMS mixture. As the IPA boiled/evaporated off, dispersed SWNTs were transferred to the PDMS base compound. After securing SWNT/IPA addition, the mixture was heated for additional $2 \mathrm{~h}(\sim 12 \mathrm{~h}$ total heating time). The PDMS base compound/SWNT mixture was then removed from heat and allowed to cool. The mixture was divided and diluted with pristine PDMS base compound (which also went through the evaporative mixing process, but with plain IPA - no SWNTs) as necessary in order to obtain desired lower $w t \%$ concentrations. As opposed to the SWNTs that were difficult to disperse, TEMs are well suited for shear mixing. Additionally, as evaporative mixing would have resulted in microsphere expansion, TEMs were added to the mixture following dilution. Equations (1) and (2) were used to determine grams of SWNTs and TEMs needed, respectively [equations (D1) and (D2), $m_{S W N T}$ and $m_{T E M}$, depending on $w t \%$ desired ( $m_{\text {base }}$ and $m_{\text {cross }}$ are amount of PDMS base and crosslinker). 


$$
\begin{aligned}
& m_{S W N T}=\frac{S W N T_{w t \%}\left(m_{\text {base }}+m_{\text {cross }}\right)}{\left(100 \%-S W N T_{w t \%}-T E M_{w t \%}\right)} \\
& m_{T E M}=\frac{T E M_{w t \%}\left(m_{\text {base }}+m_{c r o s s}+m_{S W N T}\right)}{\left(100 \%-T E M_{w t \%}\right)}
\end{aligned}
$$

Crosslinker was added to the mixture at a ratio of 10:1 (PDMS base compound/crosslinker) and shear mixed for a further $\sim 5 \mathrm{~min}$. Finally, mixtures were placed in a vacuum chamber and degassed for $\sim 30$ min to removed trapped air bubbles. 


\section{APPENDIX E}

\section{EMBEDDED CIRCUIT RESPONSE CALCULATIONS:}

While many different combinations are possible, the following calculations show an example of using IR-induced expansion of the embedded parallel resistor network shown in Figure 6.11 to either raise, lower, or maintain total resistance. Assume a circuit is fabricated with two TEM-based resistors $\left(R_{1}\right.$ and $\left.R_{2}\right)$ of identical microsphere $w t \%$ but different nanotube loading. Initially, total resistance of the circuit $\left(R_{\text {total }}\right)$ is given by equation $(\mathrm{E} 1)$.

$$
R_{\text {total }}=\frac{R_{1} R_{2}}{R_{1}+R_{2}}
$$

Because specific circuit responses are desired, careful resistor selection and design is required. Based on results from Figure $6.8(\mathrm{a}), R_{1}$ will be chosen with a SWNT $w t \%$ that falls into region 1 , likewise $R_{2}$ will be from region 2 . Because region 1 selection results in $R_{f} / R_{0}<1$ when resistor $R_{1}$ (and $R_{1}$ only) is subjected to IR-induced expansion, $R_{1}$ and therefore $R_{\text {total }}$ will decrease. Similarly, since region 2 results in a $1<R_{f} / R_{0}<10$, IR illumination of $R_{2}$ (and $R_{2}$ only) will result in an associated rise in $R_{\text {total }}$. If both $R_{1}$ and $R_{2}$ are illuminated, the circuit can be balanced such that expansion-induced resistance changes cancel each other out, and $R_{\text {total }}$ remains constant. 


\section{SELECTION OF SWNT WT\%:}

Figure 6.8(a) gives equation (E2), which rearranged to solve for $\rho$ yields equation (E3).

$$
\begin{aligned}
R_{f} / R_{0} & =0.8 e^{0.15 \rho} \\
\rho & =\frac{1}{0.15} \ln \left(\frac{R_{f}}{0.8 R_{0}}\right)
\end{aligned}
$$

In order to create a balanced circuit, $R_{1}$ is chosen such that $R_{f} / R_{0}=0.9$ (region 1 ), and $R_{2}$ such that $R_{f} / R_{0}=1.125$ (region 2). Solving for $\rho$ for each resistor results in $\rho$ of $0.785 \mathrm{k} \Omega-\mathrm{m}\left(R_{1}\right)$ and $2.273 \mathrm{k} \Omega-\mathrm{m}\left(R_{2}\right)$. Equation (E4) is the $\rho$-wt\% relationship derived from the evaporative dispersion method [Figure 6.2(b)]. Rearranging to solve for $w t \%$ yields equation (E5).

$$
\begin{aligned}
\rho & =1.7 \times 10^{-5} e^{\left(\frac{2.75}{w t \%+0.025}\right)}+0.2 \\
w t \% & =\frac{2.75}{\ln \left(\frac{\rho-0.2}{1.7 \times 10^{-5}}\right)}-0.025
\end{aligned}
$$

Solving for $R_{1}$ and $R_{2}$ gives loadings of 0.24 and $0.21 w t \%$, respectively.

\section{DESIGNING $\mathrm{R}_{1}$ AND $\mathrm{R}_{2}$ FOR A BALANCED CIRCUIT:}

When both resistors are expanded, the circuit should maintain its original $R_{\text {total }}$. While the two resistors have different $R_{f} / R_{0}$ and thus different $\rho$ values, to achieve a balanced response equal original resistances $\left(R_{10}=R_{20}\right)$ are needed. Additionally, a constraint is imposed of equal cross-sectional areas $\left(A_{1}=A_{2}\right)$. Setting $R_{1}$ and $R_{2}$ equal to 
each other [equation (E6)], substituting Pouillet's law [equation (E7)], and solving for a length relationship yields equation (E8).

$$
\begin{aligned}
R_{10} & =R_{20} \\
\rho_{1} \frac{L_{1}}{A} & =\rho_{2} \frac{L_{2}}{A} \\
L_{1} & =2.9 L_{2}
\end{aligned}
$$

Therefore, resistor $R_{1}$ should be comprised of $0.24 w t \%$ SWNT, $31.2 w t \%$ TEM, and length $2.9 L_{2}$. Resistor $R_{2}$ should be $0.21 w t \%$ SWNT, $31.2 w t \%$ TEM, and length $L_{2}$.

\section{CIRCUIT RESPONSE: IR ILLUMINATION OF $\mathrm{R}_{1}$ ONLY:}

With resistor parameters determined, evaluate circuit response upon expansion of $R_{1}$ only. Note that since $R_{10}=R_{20}$, initial total resistance [equation (E1)] simplifies to $R_{\text {total }, 0}=0.5 R_{0}$. Additionally, because of the region 1 selection, $R_{1 f} / R_{0}=0.9$. Therefore $R_{1 f}$ $=0.9 R_{0}$. Substituting this into equation (E1) and solving yields equations (E9) and (E10):

$$
\begin{aligned}
& R_{\text {total }, f}=\frac{\left(0.9 R_{0}\right) R_{2}}{\left(0.9 R_{0}\right)+R_{2}} \\
& R_{\text {total, } f}=0.47 R_{0}
\end{aligned}
$$

Since $R_{\text {total, }}<R_{\text {total }, 0}$, illumination of $R_{1}$ only causes a decrease in overall circuit resistance.

\section{CIRCUIT RESPONSE: IR ILLUMINATION OF R $\mathrm{ONN}_{2}$ :}

Similar to preceding calculations, circuit response upon expansion of $R_{2}$ only is determined. Because of the region 2 selection, $R_{2 f} / R_{0}=1.125$, therefore $R_{2 f}=1.125 R_{0}$. Again, substituting this into equation (E1) and solving yields equations (E11) and (E12): 


$$
\begin{aligned}
& R_{\text {total,f }}=\frac{R_{1}\left(1.125 R_{0}\right)}{R_{1}+\left(1.125 R_{0}\right)} \\
& R_{\text {total }, f}=0.53 R_{0}
\end{aligned}
$$

Since $R_{\text {total, }}>R_{\text {total }, 0}$, illumination of $R_{2}$ only causes an increase in overall circuit resistance.

\section{CIRCUIT RESPONSE: IR ILLUMINATION OF BOTH R AND R $_{2}$ :}

The final possible circuit response is obtained when both $R_{1}$ and $R_{2}$ are IR expanded. Substituting known values into equation (E1) and solving yields equations (E13) and (E14):

$$
\begin{aligned}
& R_{\text {total }, f}=\frac{\left(0.9 R_{0}\right)\left(1.125 R_{0}\right)}{\left(0.9 R_{0}\right)+\left(1.125 R_{0}\right)} \\
& R_{\text {total,f }}=0.5 R_{0}
\end{aligned}
$$

Since $R_{\text {total, } f}=R_{\text {total }, 0}$, illumination of both $R_{1}$ and $R_{2}$ serves to maintain overall circuit resistance. 


\begin{abstract}
APPENDIX F
PID CONTROL LOOP RESTRICTIONS:

Due to ability to independently control each LED's intensity, six diodes per axis represents $2.8 \times 10^{14}$ possible intensity/temperature combinations [or $n^{m}$, where $n=$ number of PWM steps (256), and $m=$ number of diodes (6)]. However, simplification was required for PID loop programming; and therefore LED control restrictions were implemented. The PID loop was allowed to choose between $-100 \%$ to $+100 \%$ intensity for each axis starting with the innermost diodes and working outwards. Stated another way, the PID controller would first use the innermost diodes to control the stage position [diodes labeled as "C" in Figure 7.3(c)], then set the middle diodes, and finally, if needed, set the outer diodes. For example, when the innermost diode was at $100 \%$ duty cycle ( $+33 \%$ axis control from the PID loop) and additional stage movement was needed, the innermost diode "C" would remain at $100 \%$, and intensity of the middle diode "B" adjusted. In this manner, control axis states available to the PID loop were limited to $\sim 1.5 \times 10^{3}$ (765 positive states: 255 per diode for 3 diodes, and 765 negative states). This simplifies operation as well as gives the software enough flexibility to correct thermal drift and hysteresis to maintain ordered position. Equations (F1) and (F2) are examples of positioning diode intensity calculations for a hypothetical positive axis process variable $\left(R_{P I D}\right)$ request from the PID loop (i.e., an axis request $>0 \%$ and $\left.\leq+100 \%\right)$, where $\varepsilon$ is a conversion factor based on control restrictions.
\end{abstract}




$$
\begin{aligned}
& \varepsilon=7.65 R_{P I D}
\end{aligned}
$$

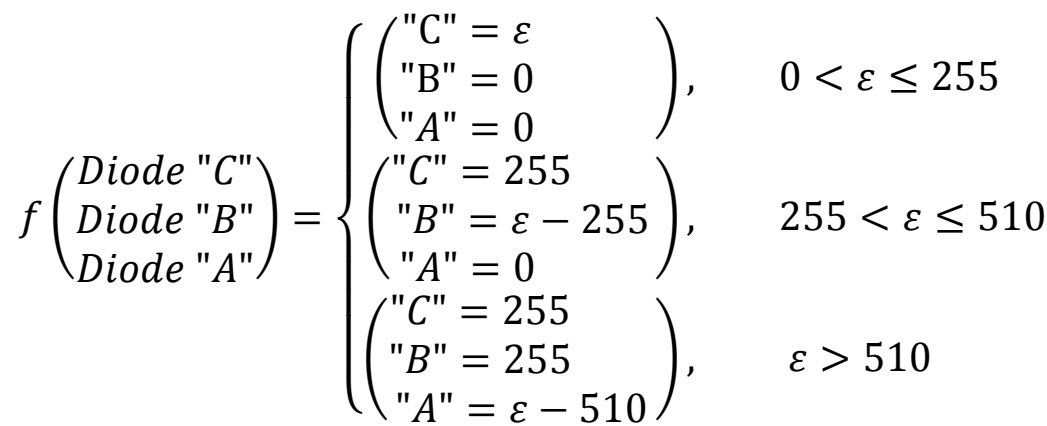




\section{APPENDIX G}

\section{COARSE ADJUST AND FINE ADJUST LEDS:}

The ability to dynamically modulate IR diode intensity results in a correlating temperature modulation in composite actuators and provides the mechanism for control of polymeric chain contraction/extension. As the number of intensity steps is fixed by the control system (at 256), the resolution of available temperature change steps is governed by the IR LED's maximum intensity specification. For example, if a high-power positioning diode ("coarse adjust") is replaced with a diode half as intense ("fine adjust"), temperature control resolution doubles, allowing for more accurate stage positioning. However, the increase in control comes at the expense of total stage travel. To quantitatively determine response differences between diodes, a maximum displacement test was conducted. This test involved measuring maximum possible stage displacement (first with all three positive, then all three negative diodes at $100 \%$ duty cycle). The absolute values of the positive and negative displacements were averaged to determine overall response. Figures 7.5(a) and (b) present detailed displacement versus time data during a single run.

Stage displacement from a single diode was verified to be $\sim 1 / 3$ of the maximum displacement [as Figure 7.5(c) shows], demonstrating actuation responses are confined to 
localized composite regions directly above each IR LED. Isolated response regions are a result of selecting positioning diodes with narrow beam angles as well as using GNP wt\%'s less than percolation threshold. Therefore, the total stage movement is a summation of all local responses, and each diode contributing to the overall response without affecting its neighboring diodes. 


\section{APPENDIX H}

\section{EFFICIENCY CALCULATIONS:}

In order to determine heat transfer into composites, an equation to model-specific heat as a function of GNP loading was needed. At low GNP loading (less than the percolation threshold), stacks of graphene plates are isolated from each other and do not form a continuous network. Therefore, the rule of mixtures was used [equation (H1)] to calculate specific heat $\left(c_{p}\right)$ for composites using $\sim 1.46 \mathrm{~kJ} / \mathrm{kg} \cdot \mathrm{K}$ for $c_{P D M S}$ and $\sim 0.5 \mathrm{~kJ} / \mathrm{kg} \cdot \mathrm{K}$ for $c_{G N P}$.

$$
c_{p}=c_{G N P}(w t \% / 100)+c_{P D M S}(1-w t \% / 100)
$$

To evaluate relationships between GNP loading and efficiency, one end of the composite strip was fixed. A $100 \mathrm{~g}$ weight was hung from the other end. Initial length was recorded, and the strip exposed to an IR source. Due to pre-strain induced from the weighted end, composite strips contracted upon IR exposure. This setup was repeated for GNP loadings from 0 to $2 \mathrm{wt} \%$. Using previously determined $c_{p}$ values, heat $\operatorname{transfer}(Q)$ and work $(W)$ were calculated using $Q=m c_{p} \Delta T$ and $W=F d$ respectively. 


\section{APPENDIX I}

\section{TESTING SEQUENCES:}

In order to characterize nanopositioner response to various composite GNP loadings, thicknesses, and types of positioning diodes, a series of automated tests were conducted. These tests and associated settings were incorporated into the LabVIEW software discussed previously, which handled all sequencing and data logging. Types of tests included (1) determining the maximum displacement possible for a given diode/composite combination, (2) determining the percent contribution to this maximum displacement from each diode, (3) measuring stage displacement accuracy and resolution (in automatic control mode) to a sequence of ordered commands, (4) long-term composite degradation testing, (5) nanopositioner response at various vacuum levels, and (6) composite thermal efficiency testing. Steps are listed below.

\section{(1) MAXIMUM DISPLACEMENT TEST:}

a) Set control mode to manual (turns off PID loop).

b) Zero stage and wait for steady state conditions (120 s typically).

c) Shift to control mode to automatic (turns on PID loop), set command position value of $0 \mu \mathrm{m}$.

d) Initiate data recording.

e) $\log 120 \mathrm{~s}$ of data at command position $0 \mu \mathrm{m}$.

f) Turn all three positive $x$ axis diodes to $100 \%$ duty cycle.

g) Wait for stage to achieve maximum displacement position (180 s typically).

h) Secure data recording. 
i) Resume automatic control mode with command position of $0 \mu \mathrm{m}$.

j) Secure test.

k) Repeat steps (a) thru (j) for $-x$ displacement, $+y$ displacement, and $-y$ displacement.

(2) INDIVIDUAL DIODE DISPLACEMENT TEST:

a) Set control mode to manual (turns off PID loop).

b) Zero stage and wait for steady state conditions (120 s typically).

c) Initiate data recording.

d) $\log 60 \mathrm{~s}$ of data with all diodes $0 \%$ duty cycle.

e) Set diode D1A to $100 \%$ duty cycle.

f) Wait for stage to achieve maximum displacement position (60 s typically).

g) Secure data recording.

h) Set diode D1A to $0 \%$ duty cycle.

i) Secure test.

j) Repeat steps (a) thru (i) for all 12 positioning diodes.

(3) SEQUENCE TEST:

a) Set control mode to manual (turns off PID loop).

b) Zero stage and wait for steady state conditions (120 s typically).

c) Shift to control mode to automatic (turns on PID loop), set command position value of $0 \mu \mathrm{m}$.

d) Initiate data recording.

e) $\log 120 \mathrm{~s}$ of data at command position $0 \mu \mathrm{m}$.

f) Set command position to desired displacement.

g) Wait $20 \mathrm{~s}$.

h) Repeat steps (f) thru (g) for each desired stage position.

i) Set command position to $0 \mu \mathrm{m}$.

j) Wait $120 \mathrm{~s}$.

k) Secure data recording.

1) Secure test.

(4) LONG-TERM COMPOSITE DEGRADATION (CYCLING) TEST:

a) Select test axis $(x$ or $y)$.

b) Initiate data recording. 
c) Run specified number of loops, each loop consisted of:

i. Run maximum displacement test.

ii. Shift to control mode to automatic (turns on PID loop).

iii. Set command position value of $0 \mu \mathrm{m}$.

iv. Wait time (typically $20 \mathrm{~s}$ ).

v. Set command position to desired displacement.

vi. Wait time (typically $20 \mathrm{~s}$ ).

vii. Repeat (iii) thru (vi) 250 times.

d) Set command position to $0 \mu \mathrm{m}$.

e) Secure data recording.

f) Secure test.

(5) VACUUM TEST:

a) Place nanopositioner in vacuum chamber and set desired pressure.

b) Allow $10 \mathrm{~min}$ stabilization/degas time.

c) Set control mode to manual (turns off PID loop).

d) Zero stage.

e) Initiate data collection.

f) Pretest stabilization delay (180 s typical).

g) Set $x$ axis to $+100 \%$ (full duty cycle on all three positive positioning diodes).

h) Wait time $(180 \mathrm{~s})$.

i) Set $x$ axis to $0 \%$ (all didoes off).

j) Wait time $(300 \mathrm{~s})$.

k) Set $x$ axis to $-100 \%$ (full duty cycle on all three negative diodes)

1) Wait time $(180 \mathrm{~s})$.

m) Set $x$ axis to $0 \%$ (all didoes off)

n) Post-test stabilization delay (180 s typical).

o) Secure data recording.

\section{(6) COMPOSITE THERMAL EFFICIENCY TEST:}
a) Cut $6 \mathrm{~mm}$ by $50 \mathrm{~mm}$ by $210 \mathrm{um}$ thick composite test strip.
b) Fix one end of strip, hang $100 \mathrm{~g}$ weight to the other end.
c) Allow to stabilize for $60 \mathrm{~s}$.
d) Record initial length.
e) Expose to IR light for $120 \mathrm{~s}$. 
f) Record change in length.

g) Attach thermocouple to center of strip.

h) Allow to stabilize for $60 \mathrm{~s}$.

i) Record temperature.

j) Secure IR illumination.

k) Secure data testing. 


\section{CURRICULUM VITAE}

NAME: $\quad$ Robert J. Loomis III

ADDRESS: Small Systems Laboratory

Department of Mechanical Engineering

University of Louisville

Louisville, KY 40292

DOB: $\quad$ Newton, New Jersey - December 28, 1978

EDUCATION

\& TRAINING: $\quad$ B.S., Mechanical Engineering

University of Michigan

1997-2001

M.S., Engineering Management

Old Dominion University

2004-2007

Ph.D., Mechanical Engineering

University of Louisville

2010-2013

AWARDS: Journal cover image: Nanotechnology, vol. 24, no. 18, May 2013.

Dean's Citation award winner, April 2013.

Two articles featured (out of only 52 included) in the 2012

Nanotechnology Highlights issue (only group to have multiple papers

featured). Per the editor-in-chief, "the highlights give a glimpse of some of the gems that the journal has had to offer over the past year".

Nanotechweb.org write-up: Air-assisted fabrication paves the way for low-cost graphene solar cells, July 2012.

Journal cover image and featured article: Nanotechnology, vol. 23, no. 21, June 2012.

Nanotechweb.org write-up: Carbon-based mixed-dimensional composites expand capability of adaptronics, May 2012. 
Nanotechweb.org write-up: Graphene-based remote controlled actuators put to the test, January 2012.

Publishers Pick: Nanotechnology (Highlighted article selected by the Nanotechnology editorial board that they feel is of particular interest to readers), January 2012.

PROFESSIONAL SOCIETIES:

PUBLICATIONS: $\quad$ Loomis, J., Xu, P., Fan, X., Fletcher, M., Cohn, R. W. \& Panchapakesan, B. Graphene/elastomer composite-based photothermal nanopositioners. Nature Scientific Reports. (in press).

Loomis, J., Xu, P. \& Panchapakesan, B. Stimuli-responsive transformation in carbon nanotube/expanding microspherepolymer composites. Nanotechnology. 24, 185703 (2013).

Xu, P., Loomis, J., Bradshaw, R. D. \& Panchapakesan, B. Load transfer and mechanical properties of chemically reduced graphene reinforcements in polymer composites. Nanotechnology. 23, 505713 (2012).

Xu, P., Loomis, J., King, B. \& Panchapakesan, B. Synergy among binary (MWNT, SLG) nano-carbons in polymer nano-composites: a Raman study. Nanotechnology. 23, 315706 (2012).

Loomis, J. \& Panchapakesan, B. Large photocurrents in airassisted deposition of single layer graphene: Effects of diffusion and drift. Nanotechnology. 23, 265203 (2012).

Xu, P., Loomis, J. \& Panchapakesan, B. Photo-thermal polymerization of nanotube/polymer composites: Effects of load transfer and mechanical strength. Applied Physics Letters. 100, 131907 (2012).

Loomis, J. \& Panchapakesan, B. Dimensional dependence of photomechanical response in carbon nanostructure composites: a case for carbon-based mixed-dimensional systems.

Nanotechnology. 23, 215501 (2012).

Loomis, J., King, B. \& Panchapakesan, B. Layer dependent mechanical responses of graphene composites to near-infrared light. Applied Physics Letters. 100, 073108 (2012). 
Loomis, J., et al. Graphene nanoplatelet based photomechanical actuators. Nanotechnology. 23, 045501 (2012).

Panchapakesan, B., Xu, P. \& Loomis, J. Carbon based micro- and nano-opto-mechanical systems (C-MOMS/NOMS). SPIE Proceedings Nano-Opto-Mechanical Systems, 2011, p. 8107.

CONFERENCE

PRESENTATIONS: Loomis, J. \& Panchapakesan, B. Carbon based dimensional composites. Tech Connect World 2012 Proceedings. 1, 286-289 (2012).

Loomis, J., King, B. \& Panchapakesan, B. Near-infrared light induced mechanical responses in single-to-few layer graphene. Tech Connect World 2012 Proceedings. 1, 294-297 (2012).

Loomis, J., et al. Photomechanical response in graphene nanoplatelet composites. Tech Connect World 2012 Proceedings. 1, 290-293 (2012).

Poster presentation, "Advanced MEMS grayscale lithography mask generation software," at 16th Annual KY EPSCoR statewide conference, May 2011.

Xu, P., Loomis, J., King, B. \& Panchapakesan, B. Synergism in binary (MWNT, SLG) nano-carbons in polymer nano-composites: a Raman study. 2012 Materials Research Society Proceedings. (proof being prepared).

Panchapakesan, B., Caprara, R., Velasco, V., Loomis, J., King, B., Xu, P., Burkhead, T., Sethu, P., Stallons, L. J., McGregor, W. G., Rai, S. N., Kloecker, G., \& Wickstrom, E. Micro- and nanotechnology approaches for capturing circulating tumor cells. Cancer Nanotechnology, 1, 3-11 (2010).

INVITED

PRESENTATIONS: Oral presentation. LabVIEW-based automated test station design. at General Electric (GE) Appliance Park LabVIEW users group quarterly meeting, November 2011.

Oral presentation. Grayscale lithography. at University of Louisville cleanroom client monthly meeting, September 2011. 
Oral presentation. Standing wave probe microlithography on self-assembled monolayers. at Joint American Society of Mechanical Engineers (ASME) Local-Section/Student Section Meeting, March 2011.

\section{PROVISIONAL} PATENTS:

Loomis, J., Cohn, R. W., \& Panchapakesan, B.

Graphene/elastomer composite-based photo-thermal nanopositioners, in progress. (2013).

Loomis, J., \& Panchapakesan, B. Stimuli-responsive polymer composites, United States Provisional Patent Application Serial No. 61/762,658. (2013).

Loomis, J., McKenna, C., \& Walsh, K. Automated generation of mask file from three dimensional model for use in grayscale lithography, United States Provisional Patent Application Serial No. 61/638,279 